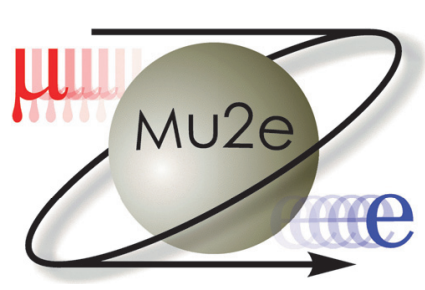

\title{
An updated estimate of the Mu2e experiment sensitivity
}

\author{
Giovanni De Felice \\ Particle Physics Student at Univeristy Of Pisa \\ Master Degree Thesis
}

supervised by

Dr. Stefano Di Falco

October 20, 2020 


\begin{abstract}
The Mu2e experiment at Fermilab will search for the conversion of a negative muon into an electron inside the field of a nucleus. This process does not conserve charged-lepton flavour and is heavily suppressed in the Standard Model (SM), with a branching ratio $<10^{-50}$. Any evidence of it would be an undeniable evidence of new physics beyond the SM.

The project sets out to achieve a single event sensitivity of $\sim 3 \times 10^{-17}$ on the ratio between the probability for a conversion of a negative muon into an electron and the one for a muon capture by the nucleus. Such a sensitivity would represent a 4 orders of magnitude improvement on the previous upper limit for the process, making possible to test predictions of different extensions of the SM.

Mu2e uses three superconducting solenoids to produce and measure the muon conversions. In the first solenoid, the Production Solenoid, pions and kaons are produced, together with other secondary products, by $8 \mathrm{GeV}$ kinetic energy proton interactions in a tungsten target. A gradient magnetic field is specifically designed to direct low momentum particles into the Transport Solenoid, an S-shaped magnet that filters out particles with unwanted charge and momentum. Muons, produced by pion and kaon decays, finally reach the aluminum Stopping Target in the Detector Solenoid, where they eventually stop and convert. The result of the conversion process is a monochromatic electron of $\sim 105 \mathrm{MeV} / \mathrm{c}$ momentum. The Detector Solenoid also hosts the two main detectors: a straw tube tracker and two CsI calorimeter disks, both providing measurement of event kinematics. A germanium detector and a LaBr crystal are located downstream of the Detector Solenoid to measure the $\mathrm{X}$ and gamma rays produced by the muon captures in the Stopping Target. A veto system of scintillators covering the Detector Solenoid and half of the Transport Solenoid is used to identify and reject cosmic rays interactions.

With respect to the initial project, the Mu2e running plan has evolved to a staged configuration with 2 years at reduced intensity before the 2025 accelerator shutdown for the neutrino beam upgrade and 2 or 3 years at full intensity after that. This, together with geometry changes and a better knowledge of detector performances obtained by the first slice tests, has required a full revision of the signal over background selection that is the subject of this thesis.

In order to achieve a new estimate of Mu2e sensitivity, the simulation of the data corresponding to the first 2 years of data acquisition has been performed. This includes both conversion electrons (CE) and the main sources of background: cosmics, decay-inorbits (DIO), radiative pion captures (RPC) and antiprotons. The characteristics of the signal and of the main backgrounds have been studied to define the best selection variables for CE.

A special effort has been devoted to the evaluation of the antiproton background. The lack of experimental data for antiproton production cross section makes the systematic uncertainty on this background significant. A new parameterization of the cross section has been developed to fit the existing data and to provide a more reliable estimate of the systematic uncertainty by comparing the results of the old and the new model. A special effort has been devoted to the optimization of the antiprotons Monte Carlo generator. This study has also revealed that the dominant component within this background is represented by antiprotons produced in the opposite direction with respect to the Transport Solenoid entrance and then redirected to it by back-scattering processes in the Production Target.
\end{abstract}


This ultimately highlights the sensitivity of the background estimate to the G4 handling of antiproton interactions in the $1 \div 3 \mathrm{GeV} / \mathrm{c}$ momentum range.

Finally, the final experimental sensitivity has been studied. The momentum and time selection have been optimized to obtain the 5-sigma discovery reach or, in case of no signal, the upper limit on conversion probability.

The results confirm that, in the first two years of data taking, Mu2e will be able to improve the current experimental sensitivity for muon-to-electron conversion in an atomic field by more than 3 order of magnitudes. 


\section{Contents}

1 Search for New Physics in the lepton flavour sector 3

1.1 The lepton sector of the Standard Model . . . . . . . . . . . . . . . . . 4

1.1.1 Accidental lepton flavour conservation in absence of massive neutrinos 4

1.1.2 CLFV theoretical framework . . . . . . . . . . . . . . 6

1.2 Experimental features of the muon channel in CLFV searches . . . . . . . 9

$1.2 .1 \mu^{+} \longrightarrow e^{+} \gamma \ldots \ldots \ldots \ldots \ldots \ldots \ldots$

$1.2 .2 \mu^{+} \longrightarrow e^{+} e^{-} e^{+} \ldots \ldots \ldots \ldots \ldots 13$

$1.2 .3 \mu^{-} N \rightarrow e^{-} N \ldots \ldots \ldots \ldots \ldots \ldots \ldots$

1.2.4 The tau channel . . . . . . . . . . . . . . . . . . 18

2 The Mu2e experiment $\quad 20$

2.1 Experimental layout: magnets and detectors . . . . . . . . . . . . . 20

2.2 The proton beam line . . . . . . . . . . . . . . . . . 21

2.2.1 Beam intensity profile . . . . . . . . . . . . . . . 24

2.2 .2 Mu2e beam structure . . . . . . . . . . . . . . . . . . 24

2.2 .3 Extinction System . . . . . . . . . . . . . . . . . 26

2.2.4 The Extinction Monitors . . . . . . . . . . . . . . 26

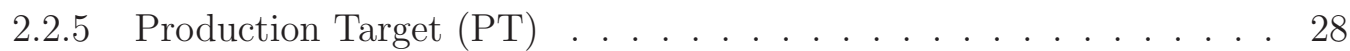

2.3 The muon beam line . . . . . . . . . . . . . . . . . . . . . 29

2.3.1 Production Solenoid (PS) . . . . . . . . . . . . . . . 29

2.3.2 Transport Solenoid $(\mathrm{TS})$. . . . . . . . . . . . . . . . . . . . . . 29

2.3.3 Detector Solenoid (DS) . . . . . . . . . . . . . . . . . 31

2.3.4 Stopping Target (ST) . . . . . . . . . . . . . . . . . . . . . . . . . . . . . 32

2.3.5 Inner and Outer Proton Absorbers . . . . . . . . . . . . . . 33

2.4 The Mu2e Tracker . . . . . . . . . . . . . . . . . . . . . . . . . . . 33

2.4 Requirements . . . . . . . . . . . . . . . 33

2.4.2 The straw tubes structure . . . . . . . . . . . . . 34

2.4 .3 Readout system . . . . . . . . . . . . . . . . . . 34

2.4.4 Resolutions . . . . . . . . . . . . . . . . . . 35

2.5 Mu2e detectors: Calorimeter . . . . . . . . . . . . . . . . . . . . . . . . . . . . . 36

2.5.1 Tasks and requirements . . . . . . . . . . . 36

2.5.2 Calorimeter structure . . . . . . . . . . . . . . . 36

2.5.3 Readout system . . . . . . . . . . . . . . . . 36

2.5.4 Resolutions ....................... 37

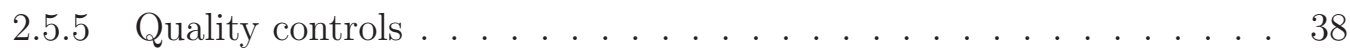

2.6 Reconstruction . . . . . . . . . . . . . . . . . . . . . . . . . . . 41

2.7 Mu2e detectors: Stopping Target Monitor . . . . . . . . . . . . . . . . 42

2.8 Mu2e detectors: Cosmic Ray Veto . . . . . . . . . . . . . . . . . . . . . . . 44 
3 Signal and background analyses $\quad 46$

3.1 The CE signal . . . . . . . . . . . . . . . . . . . . . . . . 46

3.2 Cosmic Ray Background . . . . . . . . . . . . . . . . . . . . . . 47

3.3 Decays in orbit $(\mathrm{DIO}) \ldots \ldots \ldots \ldots$. . . . . . . . . . . . . 50

3.4 Radiative pion captures $(\mathrm{RPC}) \ldots \ldots \ldots \ldots$. . . . . . . . . . . 53

3.5 Instrumental Backgrounds . . . . . . . . . . . . . . . . . . 56

3.6 Radiative muon captures $(\mathrm{RMC}) \ldots \ldots \ldots \ldots$. . . . . . . . . . 57

3.7 Variables for CE selection . . . . . . . . . . . . . . . . . 58

3.7.1 Geometric cuts . . . . . . . . . . . . . . . . 58

3.7 .2 Track quality . . . . . . . . . . . . . . . . . . . . 59

3.7.3 A simple Particle Identification (PID) . . . . . . . . . . . . . . 61

3.7.4 Optimized cuts and numerical values . . . . . . . . . . . . . 62

3.8 Comparison with CD3 results . . . . . . . . . . . . . . . . . 62

4 The Antiproton Background $\quad 69$

4.1 Antiproton production models . . . . . . . . . . . . . . . . 69

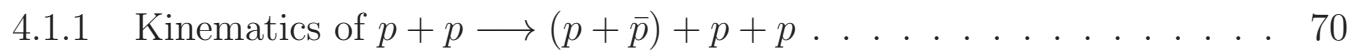

4.1.2 Available measurements for antiproton production . . . . . . . . 71

4.1.3 Striganov's model . . . . . . . . . . . . . . . . . . . . 72

4.1.4 An alternative model . . . . . . . . . . . . . . . . . 73

4.2 Antiproton simulation . . . . . . . . . . . . . . . . . . . 78

4.3 S0: Proton inelastic interactions . . . . . . . . . . . . . . . 80

4.4 S1: Antiprotons generation to TS entrance . . . . . . . . . . . . . . 81

4.4.1 Antiproton Generators . . . . . . . . . . . . . . . . . 81

4.4.2 Tracing inside the PS . . . . . . . . . . . . . . . . . 85

4.4 .3 Results after Stage 1 . . . . . . . . . . . . . . . . 87

4.5 S2: from TS entrance to COL3u exit . . . . . . . . . . . . . . 88

4.5.1 The resampling technique . . . . . . . . . . . . . . . 88

4.6 S3: from TS center to TS5 entrance . . . . . . . . . . . . . . . . . . 90

4.7 S4: from TS5 entrance to the Stopping Target . . . . . . . . . . . . . . 90

4.7.1 Origin of stopped $\bar{p}$ events . . . . . . . . . . . . . . . . . . . . 91

4.7.2 Position of stopped antiprotons in the Stopping Target . . . . . . . 92

4.7.3 Number of stopped $\bar{p}$ per proton on target . . . . . . . . . . . . . . 93

4.7.4 Check of the resampling technique . . . . . . . . . . . . . 94

4.8 S5: from the Stopping Target to the detectors . . . . . . . . . . . . . 95

4.9 S6: Reconstruction and track selection . . . . . . . . . . . . . 96

4.10 First estimate of antiproton background . . . . . . . . . . . . . 97

4.11 The antiproton generated RPC component . . . . . . . . . . . . . . . . 99

5 Optimizing the sensitivity 102

5.1 Latest Mu2e run plan . . . . . . . . . . . . . . . . . . . . . . . 102

5.2 Momentum and time distributions . . . . . . . . . . . . . . . . 103

5.3 Momentum and time selection optimization . . . . . . . . . . . . 103

5.4 Discovery-based optimization . . . . . . . . . . . . . . . . . . 104

5.5 Limit-based optimization . . . . . . . . . . . . . . . . . . . . . 108

5.6 A combined approach: the sensitivity region . . . . . . . . . . . . 109

5.7 Final background and signal expectations . . . . . . . . . . . . . . . 111 


\section{Chapter 1}

\section{Search for New Physics in the lepton flavour sector}

The Standard Model (SM) is the theory that brings together all the current knowledge on particle physics. It consists of twelve elementary fermions (and their antiparticles), grouped in three families of quarks and three families of leptons. The theory successfully describes the strong and electroweak interactions involving these particles and eventually mixing their flavour through the exchange of gauge bosons. As it comes from decades of new ideas and experimental evidences, it has now reached a surprisingly stable form with multiple recent confirmations, last being the Higgs Boson discovery at the LHC [1] [2]. However, there are still some open questions that suggest the SM is still a low-energy approximation of a more general theory: the particle mass spectrum, the hierarchy problem, the matter-antimatter asymmetry etc.

It is possible to associate to each fermion family a specific quantum number called flavour ${ }^{1}$. An intense experimental activity is currently devoted to search for anomalies in the lepton flavour sector that can hint for new physics beyond the Standard Model. Research in this field is divided into three major channels: lepton universality violation (LUV), lepton number violation (LNV) and charged lepton flavour violation (CLFV).

$$
\left(\begin{array}{l}
\nu_{e} \\
e^{-}
\end{array}\right)\left(\begin{array}{l}
\nu_{\mu} \\
\mu^{-}
\end{array}\right)\left(\begin{array}{l}
\nu_{\tau} \\
\tau_{-}^{-}
\end{array}\right)
$$

Figure 1.1: The three copies of the $\mathrm{SU}(2)$ doublet (the six anti-leptons are not shown). A flavour quantum number is associated to each family: electron number, muon number, tau number. The figure also shows the so far observed transitions within a single doublet and between different doublets (neutrino oscillations).

Lepton universality is a surprising purely experimental [3] feature of weak decays, which states that different generations share the same coupling constant and so interact in the same way, producing the same cross sections or decay amplitudes once their mass difference are taken into account. Theories predicting deviations from this empirical rule introduce new mediators such as Z' or lepto-quarks. New branches are continuously getting

\footnotetext{
${ }^{1}$ The only known difference between generations is the different strengths of their interactions with the Higgs field, known as the Yukawa couplings. This results in different masses for each particle, giving a wide range of experimental signatures.
} 
probed, for example the LHCb collaboration has recently published the first test of lepton universality in the decay of b mesons ([4]), finding no evidence for a violation. Hints of deviations from lepton universality are emerging from the $B^{+} \rightarrow K^{+} \ell^{+} \ell^{-}([5], 2.5 \sigma)$ and $\bar{B}^{0} \rightarrow D^{*+} \ell^{-} \bar{\nu}_{\ell}$ channels ([6], $2.1 \sigma$ ), leaving the field open for future developments.

Lepton number $(\mathrm{L})$ is a conserved additive quantum number representing the difference between the number of leptons and the number of antileptons in an elementary particle reaction [7]. As it happened for Barionic number (B) conservation, which turned out to be the quark number conservation, this might be an hint of a lepton hidden substructure, just not accessible to the energy available at the current accelerators. Lepton number violation is almost always associated with processes where one or two leptons are created or destroyed: well known examples are the proton decay into a (anti)lepton plus a meson and neutrino-less double beta decay (often associated to the existence of Majorana neutrino )$[8]$.

Last but not least, lepton flavour conservation has been introduced as an "accidental" conservation law to meet the experimental evidence that, for decades, no-mixing was found between different generations of leptons. The discovery of neutrino oscillations, in 1999 ([9, 10, 11]), has required a revision of the Standard Model and has naturally led to a further question, still needing a response: "Why transitions between charged leptons of different flavour are not observed?". This still unobserved process goes under the name of Charged Lepton Flavour Violation (CLFV). The next section will describe with more details how the experimental evidence of lepton flavour conservation had been implemented in the Standard Model. The minimal extension of the model to account for massive neutrinos and the consequent neutrino oscillations will also be presented. Possible channels of New Physics related to CLFV will be investigated.

\subsection{The lepton sector of the Standard Model}

\subsubsection{Accidental lepton flavour conservation in absence of mas- sive neutrinos}

The fermion contribution to the Standard Model is described by the fermion Lagrangian, which involves the kinetic terms and the Yukawa couplings of the fermion fields with the Higgs field $\Phi(x)$. This gives rise to the following gauge invariant terms [12]:

$$
\begin{gathered}
\mathcal{L}_{\text {Fermions }}=\mathcal{L}_{\text {Kin }}+\mathcal{L}_{Y} \\
\mathcal{L}_{K i n}=\sum_{\Psi=L, Q} \bar{\Psi}_{L i} \gamma^{\mu}\left(i \partial_{\mu}-\frac{1}{2} g^{\prime} \tau \cdot W_{\mu}-\frac{1}{2} g^{\prime} Y B_{\mu}\right) \Psi_{L i}+\sum_{\psi=e, u, d} \bar{\psi}_{R i} \gamma^{\mu}\left(i \partial_{\mu}-\frac{1}{2} g^{\prime} Y B_{\mu}\right) \psi_{R i} \\
\left.\mathcal{L}_{Y}=\left(Y_{u}\right)_{i j} \bar{Q}_{L i} u_{R j} \tilde{\Phi}+\left(Y_{d}\right)_{i j} \bar{Q}_{L i} d_{R j} \Phi+\left(Y_{e}\right)_{i j} \bar{L}_{L i} e_{R j} \Phi+1.1\right)
\end{gathered}
$$

In this compact notation:

- $\mathrm{i}, \mathrm{j}$ run over the three quark and lepton families. A sum over repeated indices is implied. 
- $u_{i}$ represents a generic up-type quark $\left(u_{1}=u, u_{2}=c, u_{3}=t\right), d_{i}$ the corresponding down-type quarks.

$e_{i}$ represent a generic charged lepton $\left(e_{1}=e, e_{2}=\mu, e_{3}=\tau\right)$ and $\nu_{i}$ the corresponding neutrino.

- capital letters represent left-handed (LH) fermion doublets: $Q_{L}^{T}=\left(u_{L} d_{L}\right)$ or $L_{L}^{T}=\left(\nu_{L} e_{L}\right)$, while the lower case letters stand for right-handed singlets.

- $\mathrm{SU}(2)_{L}$ indices are omitted.

- The conjugate Higgs field is defined as $\tilde{\Phi} \equiv i \tau_{2} \Phi^{*}$, where $\tau_{2}$ is the second Pauli matrix.

- $\left(Y_{f}\right)_{i j}$ are complex $3 \times 3$ matrices.

Note that, in $\mathcal{L}_{Y}$, the second lepton term including $\nu_{R}$ is missing. This is a direct consequence of the fact that neutrino mass terms are not included in this first formulation.

After the spontaneous breaking of the $\mathrm{SU}(2)_{L} \times \mathrm{U}(1)_{Y}$ symmetry caused by a non null vacuum expectation value of the Higgs field (Higgs mechanism) the Yukawa Lagrangian turns into ${ }^{2}$ :

$$
\mathcal{L}_{Y}=\bar{u}_{L i}\left(Y_{u}\right)_{i j} u_{R j}+\bar{d}_{L i}\left(Y_{d}\right)_{i j} d_{R j}+\bar{e}_{L i}\left(Y_{e}\right)_{i j} e_{R j}+\text { Higgs field interactions }
$$

It is now possible to introduce the $3 \times 3$ mass matrices, $m_{f}$, as:

$$
\left(m_{f}\right)_{i j}=\frac{v}{\sqrt{2}}\left(Y_{f}\right)_{i j}, \quad(f=\text { up, down, e })
$$

where $v$ is the vacuum expectation value of the Higgs field.

Starting from the kinetic terms, we can drop what it is not interesting for our scope and keep only the charged current terms (pure kinetic and e.m. terms have been dropped):

$$
\mathcal{L}_{C C}=\bar{u}_{L i} \gamma^{\mu} W_{\mu}^{+} d_{L i}+\bar{\nu}_{L i} \gamma_{\mu} W_{\mu}^{+} e_{L i}+\text { h.c. }
$$

And, therefore, the total Lagrangian of interest is:

$$
\mathcal{L}=\underbrace{\bar{u}_{L i} \gamma^{\mu} W_{\mu}^{+} d_{L i}}_{\mathcal{L}_{C \text { Cquarks }}}+\underbrace{\bar{\nu}_{L i} \gamma^{\mu} W_{\mu}^{+} e_{L i}}_{\mathcal{L}_{C \text { Cleptons }}}+\text { h.c. }+\underbrace{\bar{u}_{L i}\left(m_{u}\right)_{i j} u_{R j}+\bar{d}_{L i}\left(m_{d}\right)_{i j} d_{R j}}_{\mathcal{L}_{Y \text { quarks }}}+\underbrace{\bar{e}_{L i}\left(m_{e}\right)_{i j} e_{R j}}_{\mathcal{L}_{Y \text { ch.leptons }}}+\ldots
$$

It is important to notice that the fields appearing in the 1.6 are not the physical ones. If this was the case, mass terms would have appeared in the form: $m_{f} \bar{f}_{L} f_{R}$, with a scalar mass coefficient. Instead, $\left(m_{f}\right)_{i j}$ are in general non-diagonal $3 \times 3$ matrices.

Physical fields can be obtained with a change of base, i.e. the particular unitary rotations of the fields that makes the mass matrices diagonal:

$$
\left\{\begin{array}{l}
f_{R}=R_{R}^{f} f_{R}^{\prime} \\
f_{L}=R_{L}^{f} f_{L}^{\prime}
\end{array} \quad(f=\text { up, down, e, } \nu)\right.
$$

\footnotetext{
${ }^{2}$ Unitarity gauge is used. Proportionality constants have been dropped since they're irrelevant for this discussion.
} 


$$
\text { imposing: } m_{f}^{D}=R_{L}^{f \dagger} m_{f} R_{R}^{f} \quad(f=\text { up, down, e })
$$

After the rotations, the $\mathcal{L}_{c c}$ term will be in the form:

$$
\mathcal{L}_{c c}=\frac{g}{\sqrt{2}}\left(\bar{u}_{L i} \gamma^{\mu} W_{\mu}^{+}\left(R_{L}^{u \dagger} R_{L}^{d}\right)_{i j} d_{L j}+\bar{\nu}_{L i} \gamma^{\mu} W_{\mu}^{+}\left(R_{L}^{\nu \dagger} R_{L}^{e}\right)_{i j} e_{L j}\right)+\text { h.c. }
$$

Since the model needs both mass terms for up-type and down-type quarks, the four quark rotation matrices defined in 1.7 are all fixed by requiring $m_{u}$ and $m_{d}$ to be diagonal in 1.8. The misalignment $R^{u} \neq R^{d}$ gives rise to the CKM matrix $\left(M_{C K M}=R_{L}^{u \dagger} R_{L}^{d}\right)$ and the charge current term allows mixing between the $i_{t h}$ up-type quark and the $j_{t h}$ down-type quark.

For what concerns leptons, since neutrinos are massless in this model, no restrictions are set on $R^{\nu}$. The additional degree of freedom can be used to match the experimental evidence of no flavour changing transition, with the minimal construction given by $R^{\nu}=R^{e}$. This implies $R_{L}^{\nu \dagger} R_{L}^{e}=\delta_{i j}{ }^{3}$ and no mixing is allowed between different lepton generations. The resulting Lagrangian is invariant under three different $U(1)$ symmetries, corresponding to electron, muon and tau lepton number "accidental" conservation.

\subsubsection{CLFV theoretical framework}

The recent observation of neutrino oscillations calls for an extension of the SM to include neutrino mass terms and consequently break the lepton family number conservation law. This scenario is now exactly the same as quarks in Eq. 1.9 and leads to the PontecorvoMaki-Nakagawa-Sakata (PMNS) matrix defined as: $U_{P M N S} \equiv R_{L}^{\nu \dagger} R_{L}^{e}$ and to CLFV as well. As it is for the CKM matrix, the PMNS matrix also connects the down-type fermion (neutrinos, in the leptons case) flavour and mass eigenstate:

$$
\nu_{\alpha}=\sum_{k=1,2,3} U_{\alpha k} \nu_{k}, \quad \alpha=e, \mu, \tau .
$$

Let us consider the case of a muon conversion into an electron, without any neutrinos in the final state. This process can occur via different ways, the most probed being:

- $\mu^{+} \longrightarrow e^{+} \gamma$ :

- $\mu^{+} \longrightarrow e^{+} e^{-} e^{+}$:

- $\mu^{-} N \longrightarrow e^{-} N$ :

In this minimal extension of the Standard Model, CLFV may occur through a second order loop diagram involving neutrino oscillations (Fig. 1.2 shows some Feynmann diagrams for the three highlighted processes).

In order to estimate the typical branching ratio (BR) for these processes one can evaluate the two major contributions to the amplitude coming from the internal lines. Top-left diagram of Fig. 1.2 can be used as a reference for notations.

1. The first contribution comes from the sum over neutrino mass eigenstates of the fermion propagator:

$$
A_{1} \propto \sum_{i=1}^{3} \frac{U_{\mu i}^{*} U_{e i}}{k^{2}-m_{i}^{2}}
$$

\footnotetext{
${ }^{3} R^{e}$ and $R^{\nu}$ matrices are unitary since they represent rotations in the complex space.
} 

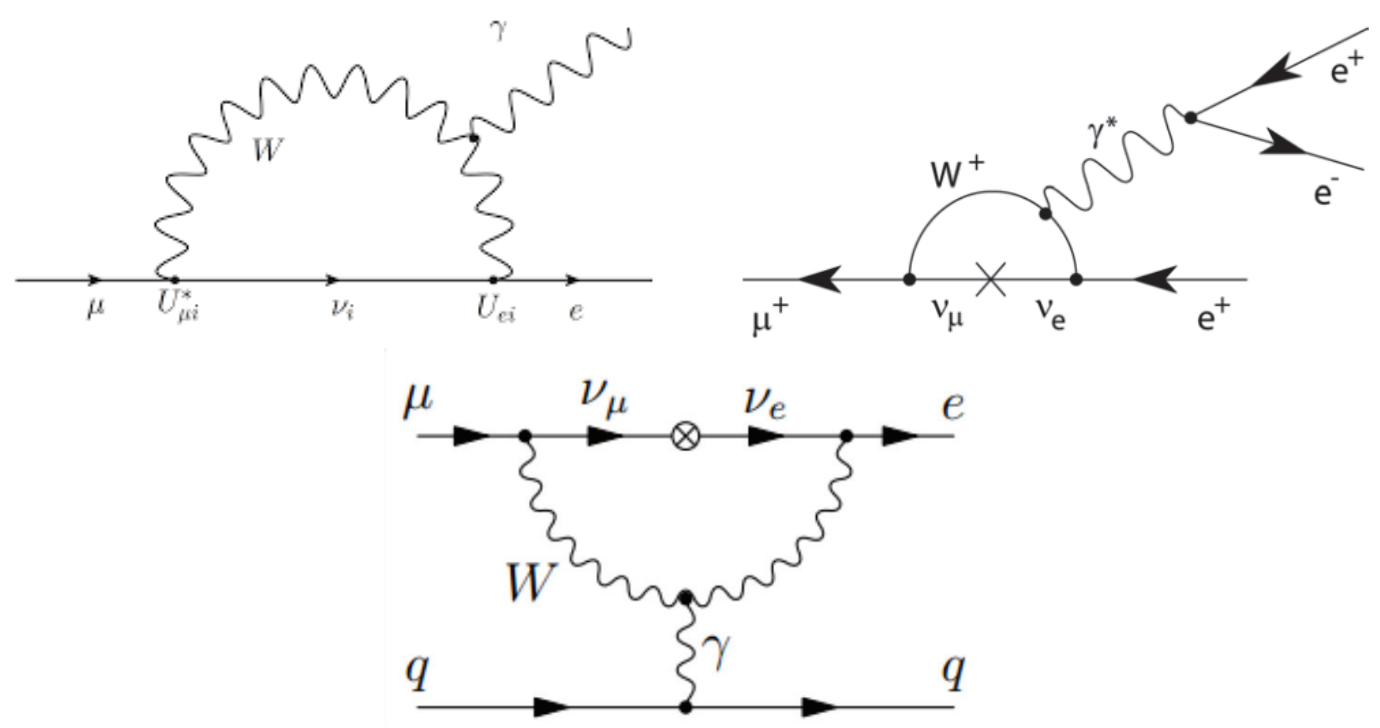

Figure 1.2: Feynman diagrams at the SM loop level of the three most experimentally probed CLFV processes. In the top left diagram the neutrino flavour eigenstates are substituted with a generic mass eigenstate $\nu_{i}$ to be consistent with the calculation. The only two relevant differences between the three processes are the number of fermions in the initial and final state and an extra e.m. vertex for $\mu^{+} \longrightarrow e^{+} e^{-} e^{+}$and $\mu^{-} N \longrightarrow e^{-} N$ with respect to $\mu^{+} \longrightarrow e^{+} \gamma$.

where $U$ is the PMNS matrix, $k$ is the sum of the of the $\mathrm{W}$ and muon 4-momenta and $m_{i}$ is the neutrino mass. Since $k^{2}>>m_{i}^{2}$ one can write:

$$
A_{1} \propto \frac{1}{k^{2}} \sum_{i=1}^{3} \frac{U_{\mu i}^{*} U_{e i}}{1-\frac{m_{i}^{2}}{k^{2}}} \sim \frac{1}{k^{2}} \sum_{i=1}^{3}\left(U_{\mu i}^{*} U_{e i}+\frac{m_{i}^{2}}{k^{2}} U_{\mu i}^{*} U_{e i}\right)
$$

that, using the unitarity of the PMNS matrix $\left(\sum U_{\mu i}^{*} U_{e i}=0\right)$, reduces to:

$$
A_{1} \propto \frac{1}{k^{4}} \sum_{i=1}^{3} U_{\mu i}^{*} U_{e i} m_{i}^{2}
$$

The unitarity of the PMNS matrix can be used again to highlight a GIM-like cancellation:

$$
\begin{gathered}
\sum_{i=1}^{3} U_{\mu i}^{*} U_{e i} m_{i}^{2}=\sum_{i=1}^{3} U_{\mu i}^{*} U_{e i} m_{i}^{2}+U_{\mu 2}^{*} U_{e 2} m_{1}^{2}-U_{\mu 2}^{*} U_{e 2} m_{1}^{2}+U_{\mu 3}^{*} U_{e 3} m_{1}^{2}-U_{\mu 3}^{*} U_{e 3} m_{1}^{2} \\
A_{1} \propto \frac{1}{k^{4}} \sum_{i=2,3}\left[U_{\mu i}^{*} U_{e i} \Delta m_{i 1}^{2}\right]
\end{gathered}
$$

A more intuitive way to get the $\Delta m_{i 1}^{4}$ dependence is to consider the neutrino flavour in the propagator: the probability of an oscillation between a muon-neutrino and an electron-neutrino comes with a $\sin ^{2}\left(\frac{1.27 \Delta m_{i j}^{2}}{E}\right)$ dependence which is proportional to $\Delta m_{i j}^{4}$ for small values of the sin argument. 
2. The second contribution directly arises from the two $\mathrm{W}$ propagators:

$$
A_{2} \propto \frac{1}{M_{W}^{4}}
$$

The module-square of the amplitude and normalization with the ordinary muon decay rate allows a rough estimate of the probability for a muon to convert into an electron:

$$
B R(\mu \rightarrow e) \simeq \frac{\Gamma(\mu \rightarrow e)}{\Gamma(\mu \rightarrow e \nu \bar{\nu})} \propto\left|\frac{\Delta m_{i 1}^{2}}{M_{W}^{4}}\right|^{2} \times\left(\frac{m_{\mu}^{5} G_{F}^{2}}{192 \pi^{3}}\right)^{-1}
$$

Using the fact that $G_{F} / \sqrt{2}=g^{2} / 8 M_{W}^{2}$ one gets:

$$
B R(\mu \rightarrow e) \propto\left(\frac{\Delta m_{i 1}}{M_{W}}\right)^{4}
$$

Considering the experimental evidence of $\Delta m_{i j}^{2}<10^{-3} \mathrm{eV}^{2}$, the resulting numerical value is of the order $10^{-50}$. This suppression factor is common to all the three shown processes. When the e.m. vertices are taken into account, an additional factor $\alpha$ for $\mu^{+} \longrightarrow e^{+} \gamma$ and $\alpha^{2}$ to both $\mu^{+} \longrightarrow e^{+} e^{-} e^{+}$and $\mu^{-} N \longrightarrow e^{-} N$ need to be applied. Limits on the $B R\left(\mu^{+} \longrightarrow e^{+} \gamma\right)$ are then a factor $\alpha$ more stringent than the ones on the other two channels.

A more accurate calculation for $\operatorname{BR}(\mu \rightarrow e \gamma)$ can be found in [13] and predicts an even smaller value, $10^{-55} \div 10^{-54}$ : about forty orders of magnitude smaller than any sensitivity of a modern experiment. As a consequence, any evidence of a CLFV signal would be a clear signal of physics beyond the Standard Model.

Possible extensions can be obtained using supersymmetric models, heavier propagators or non-renormalisable operators. Fig. 1.3 shows, as example, some Feynman diagrams at the loop level including supersymmetric particles. The resulting rates are calculated to be about $10^{40}$ times bigger than the correspondent SM ones (shown in Fig. 1.2) and are thus accessible by the present experiments.

All CLFV SM extensions can be described using the effective Lagrangian: ${ }^{4}[18]$ :

$\mathcal{L}_{C L F V}=\frac{m_{\mu}}{(k+1) \Lambda^{2}} \bar{\mu}_{R} \sigma_{\mu \nu} e_{L} F^{\mu \nu}+\frac{k}{(1+k) \Lambda^{2}} \bar{\mu}_{L} \gamma_{\mu} e_{L}\left(\bar{u}_{L} \gamma^{\mu} u_{L}+\bar{d}_{L} \gamma^{\mu} d_{L}+\bar{e}_{L} \gamma^{\mu} e_{L}\right)+h . c$.

where $F^{\mu \nu}$ is the electromagnetic field tensor, $\Lambda$ parameterizes the scale of New Physics while the $k$ parameter defines the relative contribution of the first term, involving loops and supersymmetry, with respect to the second one, a contact term with an hidden heavy propagator. The first term, similar to a magnetic dipole interaction, directly appears at the tree level in $\mu^{+} \longrightarrow e^{+} \gamma$, while is at order $\alpha$ for $\mu^{+} \longrightarrow e^{+} e^{-} e^{+}$and $\mu^{-} N \longrightarrow e^{-} N$. The second one is a four-fermion operator that mediates $\mu^{+} \longrightarrow e^{+} e^{-} e^{+}$and $\mu^{-} N \longrightarrow e^{-} N$ at the leading order, while for $\mu^{+} \longrightarrow e^{+} \gamma$ it only contributes at loop level.

Fig. 1.4 shows the regions in the $\mathrm{k}-\Lambda$ space currently excluded by the experiments at 90\% CL. The same figure presents the exclusion regions corresponding to the sensitivity planned by the next generation experiments.

\footnotetext{
${ }^{4}$ The formula is given in the muon channel context. Further possible extensions can be found in $[14,15,16]$. In particular, additional terms to the 1.15 Lagrangian can be found in [17] with both constructive and destructive interference between them.
} 

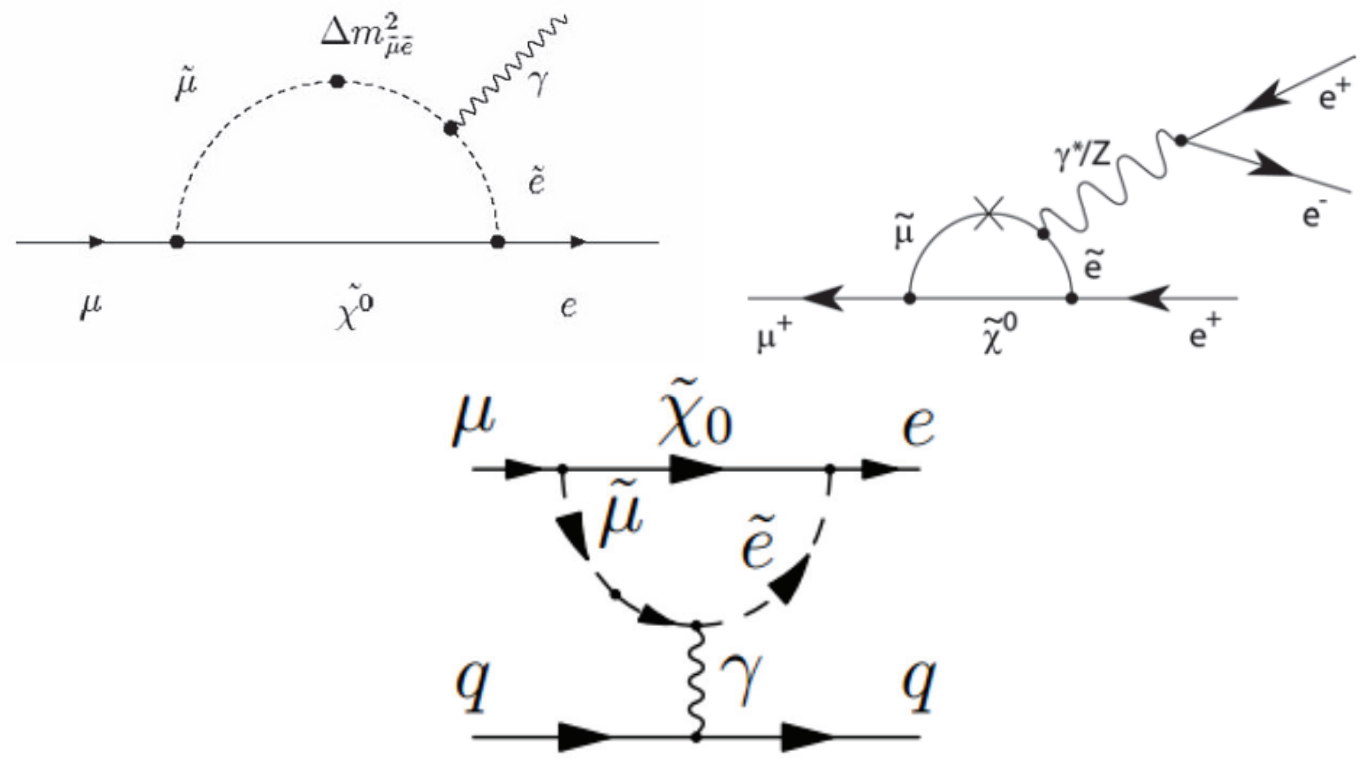

Figure 1.3: Feynman diagrams at the loop level, allowed by Supersymmetry, of the three most experimentally probed CLFV processes.

\subsection{Experimental features of the muon channel in CLFV searches}

It is interesting to remind that the first search for a CLFV process was performed in the $\mu^{+} \longrightarrow e^{+} \gamma$ channel by Hincks and Pontecorvo [19] back in 1948, right after the famous Conversi-Pancini-Piccioni experiment (1947) [20]. The absence of such a decay led to the two neutrinos hypothesis and the further first experimental evidence of a suppressed CLFV process: $\mathrm{BR}\left(\mu^{+} \longrightarrow e^{+} \gamma\right)<10^{-1}$. Since those days, the experimental search for CLFV has improved by many orders of magnitude thanks to the fast progression, especially in beam and detector technology. Fig. 1.5 graphically retraces the history of CLFV experiments.

The most promising channel is now represented by muons: their production is favored in pion and kaon decays (which are easy to obtain from hadronic interactions) and their life-time is sufficiently long to allow formation of a muon beam. The actual technology allows for muon beam intensities in the range of $10^{8} \div 10^{11} \mu / s$.

The three main CLFV channels involving muons have different sensitivities to effective lagrangian terms and present different experimental challenges: they are complementary and all necessary to measure both $\Lambda$ and $k$. The present limits on the muon sector are presented in Tab. 1.1: the experimental challenges and future perspectives for each of the three channels will be discussed in the following paragraphs.

\begin{tabular}{ccc} 
Process & Upper Limit $(90 \%$ CL) & Reference \\
\hline$\mu^{+} \longrightarrow e^{+} \gamma$ & $<4.2 \times 10^{-13}$ & 2016, MEG [21] \\
$\mu^{+} \longrightarrow e^{+} e^{-} e^{+}$ & $<1.0 \times 10^{-12}$ & 1988, SINDRUM [22] \\
$\mu^{-} \mathrm{Ti} \longrightarrow e^{-} \mathrm{Ti}$ & $<4.4 \times 10^{-12}$ & 1993, SINDRUM II [23] \\
$\mu^{-} \mathrm{Au} \longrightarrow e^{-} \mathrm{Au}$ & $<7 \times 10^{-13}$ & 2006, SINDRUM II [24]
\end{tabular}

Table 1.1: Current 90\% CL upper limits on the most sensitive CLFV muonic channels. 


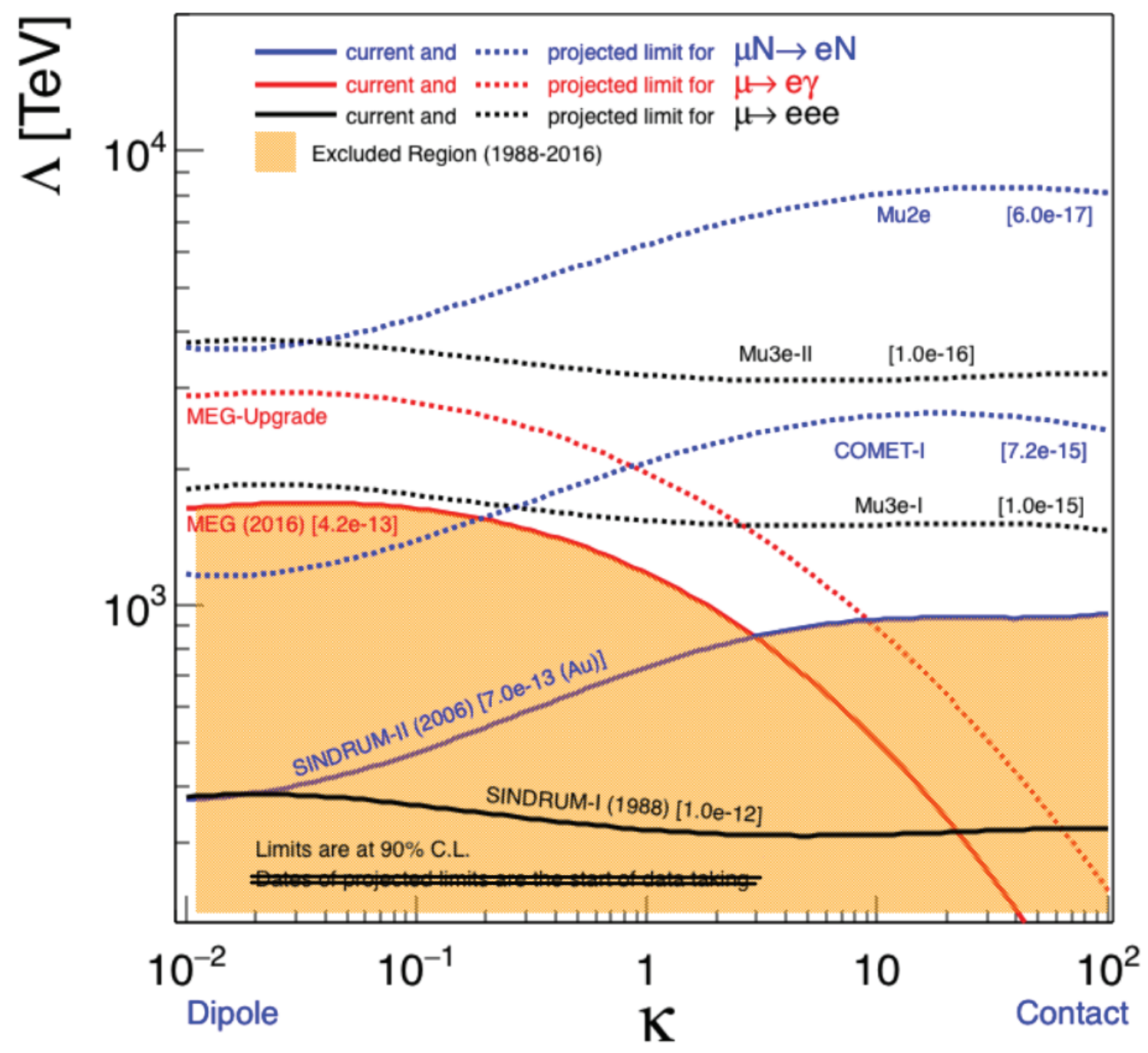

Figure 1.4: Excluded regions in the (k, $\Lambda$ ) plane for the $\mu^{+} \longrightarrow e^{+} \gamma$ (by MEG), $\mu^{+} \longrightarrow e^{+} e^{-} e^{+}$ (by SINDRUM) and $\mu^{-} N \longrightarrow e^{-} N$ (by SINDRUM-II) channels and expected exclusion for approved future experiments. $\Lambda$ sets the scale of New Physics (in $\mathrm{TeV}$ ) while $k$ defines the relative contribution of the four-fermion interaction term with respect to the dipole term, as they are defined in Eq. 1.15. Small values of $k$ make the dipole term dominating and are consequently probed better by a $\mu^{+} \longrightarrow e^{+} \gamma$ search. On the contrary, $\mu^{-} N \longrightarrow e^{-} N$ is more suitable for higher $k$ values.

\subsection{1 $\mu^{+} \longrightarrow e^{+} \gamma$}

In this case muons are stopped and decay at rest. Positive muons are preferred to the negative ones because these last may undergo nuclear capture with a significant loss of statistics ${ }^{5}$ The clear signature of the decay at rest, that is a $52.8 \mathrm{MeV}$ electron and a photon with opposite momentum emitted at the same time, characterizes $\mu^{+} \longrightarrow e^{+} \gamma$ as an almost background-free channel.

However, two little but important experimental backgrounds need to be understood:

- Radiative muon decay (RMD): $\mu^{+} \rightarrow e^{+} \gamma \nu_{e} \bar{\nu}_{\mu}$, where the neutrinos carry off a small

\footnotetext{
${ }^{5}$ For muonic atoms of heavier elements, the radius of the lowest energy orbit is comparable to the nucleus radius.
} 


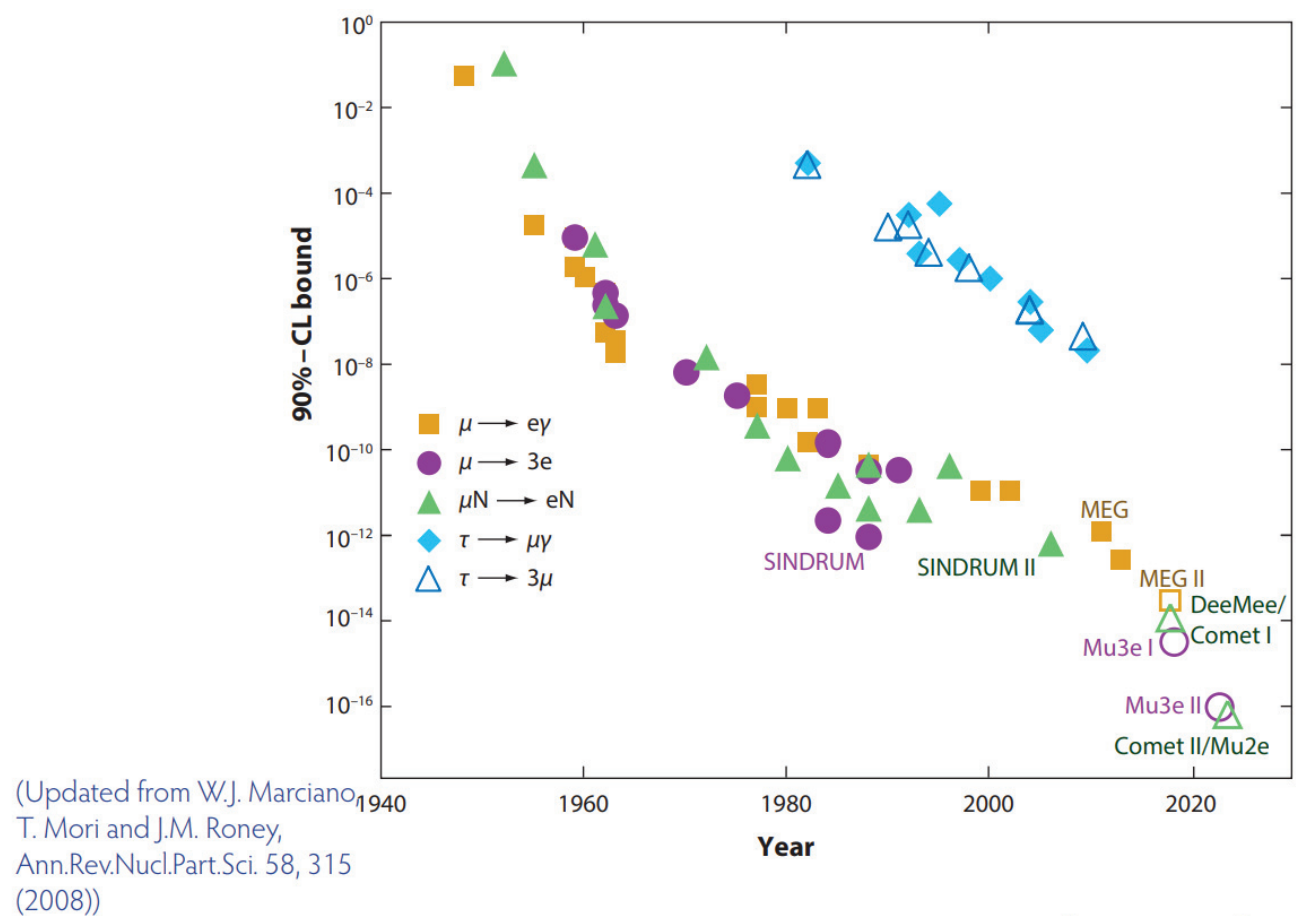

Figure 1.5: History of CLFV searches from 1948 to present days. Empty markers in the bottom-right region represent experiments which are still under construction.

fraction of the momentum. This is an "in time" intrinsic background (it then scales linearly with the muon stop rate $\Gamma_{\mu}$ ) but is only about $10 \%$ of the total background.

- Accidental background: The dominant contribution is the overlap between an ordinary Michel decay $\left(\mu^{+} \rightarrow e^{+} \nu_{e} \bar{\nu}_{\mu}\right)$ positron and a photon coming from different sources, usually started from another muon decay within the same time resolution. Some examples are: annihilation of positrons $\left(e^{+} e^{-} \rightarrow \gamma \gamma\right)$, scattering on nuclei $(e N \rightarrow$ $e N \gamma)$ and radiative muon decays $\left(\mu^{+} \rightarrow e^{+} \gamma \nu_{e} \bar{\nu}_{\mu}\right)$. The dependence of the rate of this kind of background is given by:

$$
\Gamma_{a c c} \propto \Gamma_{\mu}^{2} \cdot \epsilon_{e} \cdot \epsilon_{\gamma} \cdot \delta T_{e \gamma} \cdot \delta E_{e} \cdot\left(\delta E_{\gamma}\right)^{2} \cdot\left(\delta \Theta_{e \gamma}\right)^{2}
$$

where $\Gamma_{\mu}$ is the muon stop rate, $\epsilon_{e, \gamma}$ are the efficiencies for the positron and the photon, $\delta T_{e \gamma}, \delta E_{e, \gamma}$ and $\delta \Theta_{e \gamma}$ are respectively the time, energy and angular resolution. The sensitivity of a $\mu^{+} \longrightarrow e^{+} \gamma$ search is then driven by the photon energy and angle measurements, which appear quadratically in the 1.16.

Since both backgrounds scale with the muon stopping rate there is no gain in having a pulsed beam structure so that a continuous beam is preferred. In addition, since an higher muon stopping rate corresponds to a lower statistical error but with also a lower signal-tobackground ratio, an optimal muon stopping rate exists, depending on the experimental apparatus, that allows to minimize the total error on the measurement.

The stopping target has to be thin to reduce as much as possible the Multiple Coulomb Scattering affecting the angular resolution of the outgoing electron. On the other side it needs to be thick enough to stop a large fraction of the incoming muons. This leads to a tilted geometry like the one shown in Fig. 1.6: muons encounter enough material to 
be stopped while outgoing electrons cross a thin amount of material reducing multiple scattering.

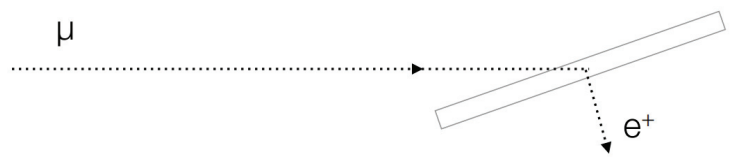

Figure 1.6: Typical sloping geometry of a target for $\mu^{+} \longrightarrow e^{+} \gamma$ searches.

Experiments involved in this search differ from each other in the way they detect the photon: it can be converted and the resulting $e^{+} e^{-}$pair can be tracked in a magnetic field to reconstruct the photon momentum, or it can be detected directly by a calorimeter. The two approaches differ in efficiency and resolution: the photon conversion has an excellent momentum resolution (it also allows a better reconstruction of the $e \gamma$ vertex ) but the efficiency is suppressed by the (order 1\%) conversion probability, this needs to take place in a very thin layer of high-Z material in order not to interfere with the $e^{+} e^{-}$pair momentum measurement; for the calorimetric approach the opposite is true.

At the present days the best limit on the $\mu^{+} \longrightarrow e^{+} \gamma$ is set by the MEG experiment at PSI (2016, [21]): $4.2 \times 10^{-13}(90 \% \mathrm{CL})$. In MEG, $7.5 \times 10^{14}$ positive muons were stopped in a graphite target; following Fig. 1.7, the positron was detected by sixteen drift chambers and several scintillators in a magnetic field while the photon energy and direction was measured thanks to a liquid Xenon calorimeter.
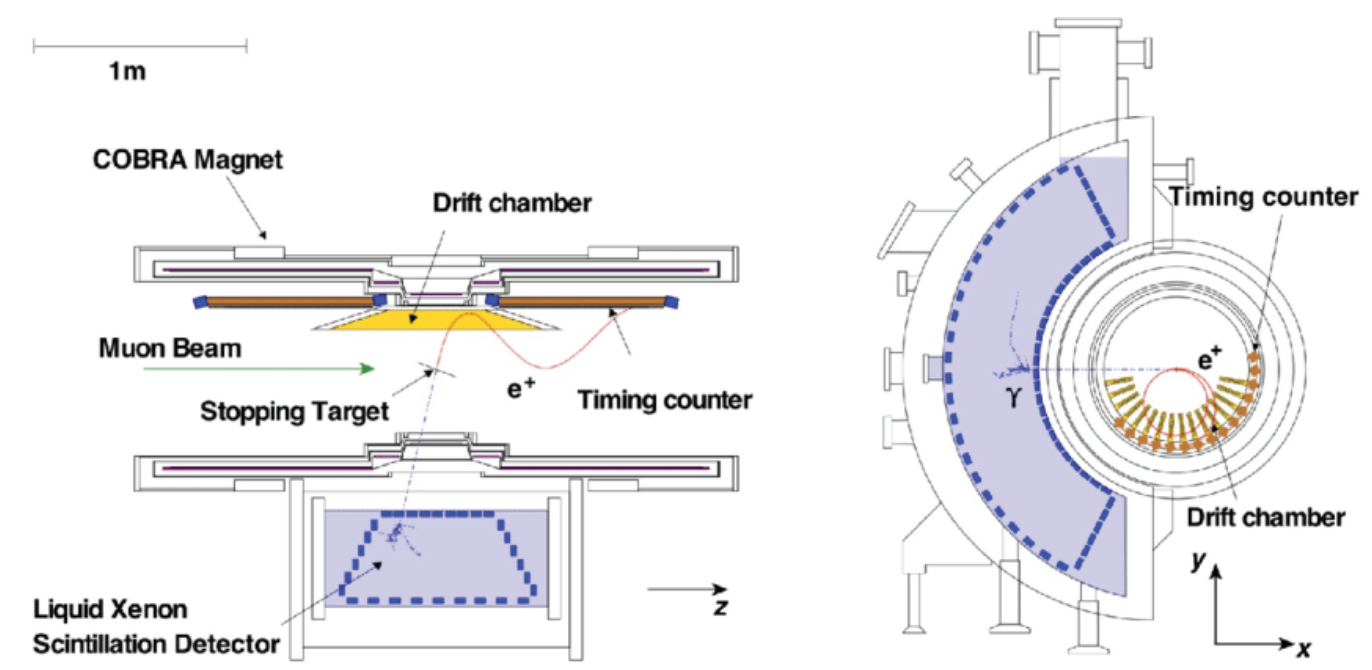

Figure 1.7: Plane and longitudinal schematic view of the MEG experiment.

An upgrade of the MEG experiment, MEG-II, is currently under construction and is expected to reach an upper limit of $\sim 6 \times 10^{-14}$ in a 3-year run starting from 2021 [25]. This will be possible by pushing the sub-detectors to meet the most advanced technologies. Unfortunately, no further improvement will be possible exploiting an increased muon beam intensity ${ }^{6}$ due to the quadratic dependence of the accidental background on the muon rate.

\footnotetext{
${ }^{6} \mathrm{PSI}$ is considering a beamline with $>10^{9} \mu / \mathrm{s}$.
} 
Putting some indicative MEG parameters inside Eq. 1.16 reveals that the effective branching ratio of accidental background is already comparable with the best presently achievable limits [12]. In order to fully exploit the PSI improved intensity and break the $10^{-15}$ wall, a novel experimental concept will be required.

\subsection{2 $\mu^{+} \longrightarrow e^{+} e^{-} e^{+}$}

Also in this case the positive muons are stopped to study their decay at rest. In the $\mu^{+} \rightarrow e^{+} e^{-} e^{+}$channel the signature is represented by two positrons and one electron coming from the same vertex and with a total energy equal to the muon mass $E_{\text {tot }} \simeq 105.6$ $\mathrm{MeV}$. Another kinematic constraint comes from the three body decay theory that requires the daughter particles to lie in the same plane with a maximum allowed energy perdaughter of about $m_{\mu} / 2$. The exact energy distribution of the daughter particles requires to know the underline physics, which is instead unknown. As a consequence, to know the percentage of particles with momentum above a fixed threshold, as it is commonly needed to determine the detector efficiencies, one needs to rely on some physical assumptions.

This channel shares a lot of similarities with $\mu^{+} \longrightarrow e^{+} \gamma$ searches, since the same final state can be reached after a pair production of the photon. As in the previous case, the main sources of background are:

- Radiative muon decay with internal conversion: $\mu^{+} \rightarrow e^{+} \gamma \nu_{e} \bar{\nu}_{\mu}$ with the radiative photon internally converting into an electron pair. The final state is then $\mu^{+} \rightarrow e^{+} e^{-} e^{+} \nu_{e} \bar{\nu}_{\mu}$. In order to be sensitive to the small energy carried off by the neutrinos an experimental resolution of $\sim 1 \mathrm{MeV}$ needs to be reached (see Fig. 1.8). This is enough to reduce this background rate to $\mathrm{O}\left(10^{-16}\right)$ [12], well below the current upper limit for this CLFV process.

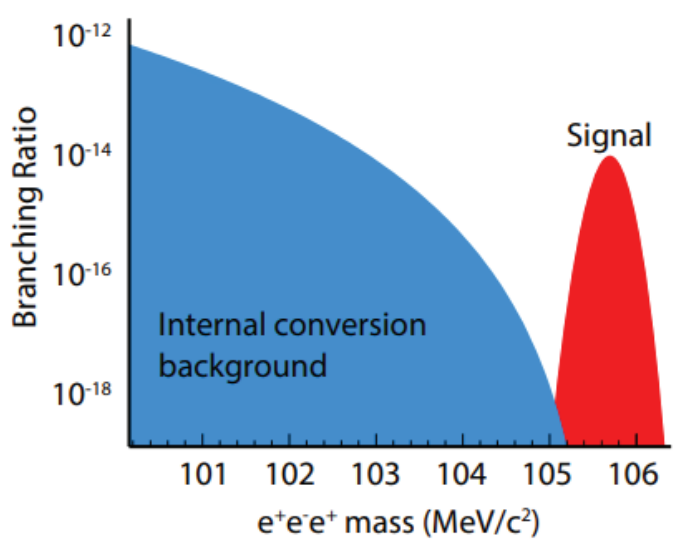

Figure 1.8: Invariant mass spectrum of the $e^{+} e^{-} e^{+}$system for radiative muon decay background and for the signal of the CLFV process.

- Accidental background: This originates from the combination of positrons and electrons coming from ordinary $\mu^{+}$decays: the same final state can be reached by one $\mu^{+}$decay and an accidental $e^{+} e^{-}$pair produced by a Bhabba scattering or a radiated photon conversion or by $2 \mu^{+}$decays $e^{-}$produced by a Compton scattering or by a mis-reconstructed pair produced by a radiated photon. As a consequence, this background scales with the square or the cube of the muon flux intensity. 
As for $\mu^{+} \longrightarrow e^{+} \gamma$, the search is optimized with a continuous beam and the sensitivity is limited by the accidental background.

The present limit for $\mu^{+} \rightarrow e^{+} e^{-} e^{+}$is $1.0 \times 10^{-12}(90 \% \mathrm{CL})$. It was set by the SINDRUM experiment at PSI (1988, [22]) where electrons and positrons from $5 \cdot 10^{6}$ positive muons were detected by five multi-wire proportional chambers and 64 scintillators in a low magnetic field.

A new experiment, called Mu3e, has already been approved at PSI and aims to reach a sensitivity of $\mathrm{O}\left(10^{-16}\right)$. This is reached with a detector made of multiple layers of silicon pixel: a cylindrical double layer close to the target and five outer double layers in the center, forward and the backward extension (1.9). All the components are immersed in a $\sim 1 \mathrm{~T}$ magnetic field and centered around a double-cone target. Two timing detector systems are also used to reject the accidental background.

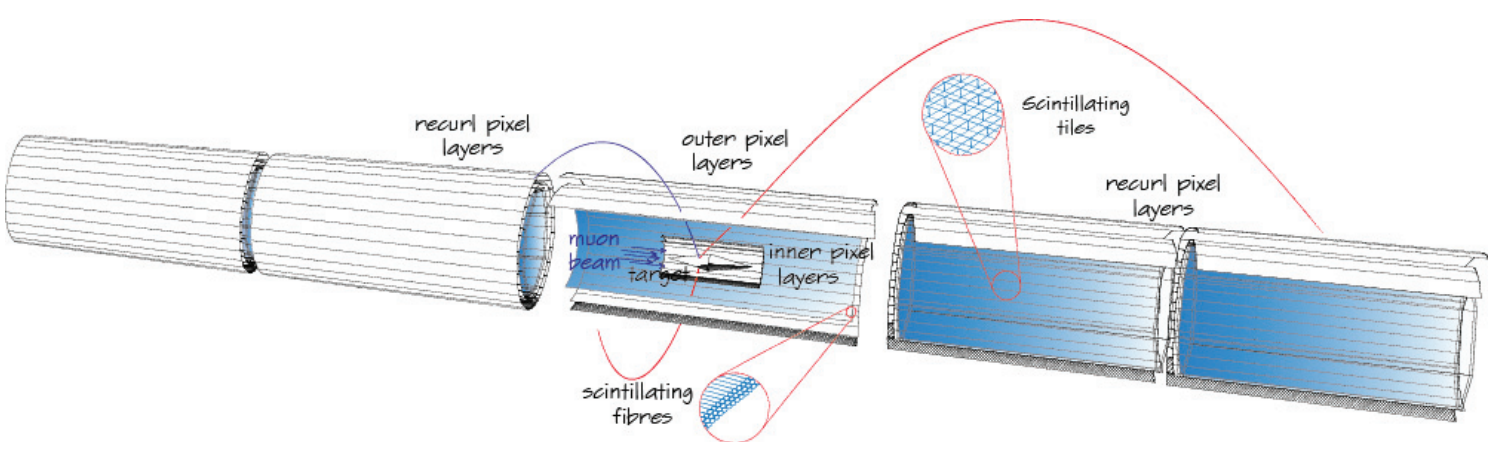

Figure 1.9: Sketch of the Mu3e detector. The geometry is optimized to allow the detection of electrons in the wide momentum window $\left[0, m_{\mu} / 2\right]$.

\subsection{3 $\mu^{-} N \longrightarrow e^{-} N$}

In this case negative muons are captured by a nucleus and their decays are observed. The muon to electron conversion consists of a muon decaying into an electron without the emission of neutrinos. Among the mentioned processes, this channel offers the most powerful sensitivity and shows some differences with respect to the previous two.

The signal is a monochromatic conversion electron (CE) with an energy equal to:

$$
E_{C E}=m_{\mu}-B_{\mu}(A, Z)-R_{N}(A, Z)
$$

with $B_{\mu} \approx Z^{2} \alpha^{2} m_{\mu} / 2$ being the muon binding energy to the atom and $R_{N} \approx m_{\mu}^{2} / 2 m_{N}$ the recoil energy of the nucleus. Since these terms are usually very small, the conversion energy is close to the muon mass and far from the endpoint of an ordinary muon decay. In addition, thanks to the one particle final state, this channel is not affected by accidental background, as it was happening in the two previous cases, and can fully benefit from a beam intensity increase. It is also usually possible to change the nucleus in order to keep related systematic uncertainties under control.

In the $\mu^{-} N \longrightarrow e^{-} N$ search, a muon beam is used to strike a heavy $\mathrm{Z}$ target. Inside it, stopped muons rapidly displace electrons of near nuclei and fall into the lowest energy level. This process is incredibly fast $\left(\mathrm{O}\left(10^{-13}\right) \mathrm{s}\right)$ and is characterized by the emission of an X-ray. In the field of the nucleus the muon can undergoes three processes: it can decay, can be captured by the nucleus itself or eventually convert forming the signal. 
The total disappearance rate, related to the total probability, is given by the sum of the contributions (the conversion probability has been dropped since it is negligible compared to the others):

$$
\frac{1}{\tau_{\text {tot }}}=\frac{1}{\tau_{\text {decay }}}+R_{\text {capture }}
$$

with $\tau_{\text {tot }}$ being the total mean life for a muon in the lowest atom shell, $\tau_{\text {decay }}$ the decay in orbit mean life and $R_{\text {capture }}$ the capture rate. Measurements of the muon lifetime, together with a valid calculation of the decay in orbit life time ${ }^{7}$. can be used in Eq. 1.18 to extract the capture rate or probability [27].

Instead of the measurement of the muon-to-electron rate, the ratio:

$$
R_{\mu e}=\frac{N(\mu \rightarrow \text { e conver sions })}{N(\text { nuclear captures })}
$$

is typically preferred. The overlap of the muon wave function with the nucleus creates several theoretical uncertainties that can be mostly canceled out with the usage of $R_{\mu e}$. The denominator can be obtained through the measurement of monochromatic X-Ray emissions that characterize the muon capture processes.

The most important background sources are:

- $\mu^{-}$decays in orbit $(\mathrm{DIO})$

The ordinary muon decay in vacuum $(\mu \rightarrow e \nu \bar{\nu})$, known as the "muon Michel decay", exhibits its kinematic endpoint at:

$$
E_{\text {max }}=\frac{m_{\mu}^{2}+m_{e}^{2}}{2 m_{\mu}}=52.8 \mathrm{MeV}
$$

also including the radiative corrections. However, the computation is different in case of a muon bound to an atom. The outgoing electron can now exchange a photon with the nucleus and the recoil can enhance the final energy. The complete calculation has been performed by Czarnecki et al. [28] and showed in Fig. 1.10. In the limit where the neutrino energies are zeros, i.e. the electron kinematic endpoint, this process exactly mimic a conversion $\mu^{-} N \longrightarrow e^{-} N$. This is an intrinsic background that scales linearly with the muon flux intensity. A precise momentum measurement turns out to be the only way to distinguish between DIOs and signal electrons. In particular, the right side tail of the resolution function must be well known.

- Cosmic Rays (CR)

Since the electron is the only particle in the final state no time coincidence can be used to identify a muon to electron conversion. For this reason this kind of experiments suffer from out of time backgrounds, the most important being the one due to cosmic rays. A cosmic ray can in fact strike any component of the apparatus and produce an electron, with a flat time distribution. To suppress cosmics background, experiments need a large and hermetic veto system covering the detectors.

- Radiative Pion Captures (RPC)

Pions contaminating the muon beam can be captured by the target nuclei and emit

\footnotetext{
${ }^{7}$ The assumption of taking the same decay mean life for muons in orbits and vacuum $\left(\tau_{D}=\tau_{0} \simeq\right.$ $2.1970 \times 10^{-6} \mathrm{~s}$ ) is not completely correct. Even if the correction is small, relativistic time dilatation for the muons average angular velocity and the phase space correction should be taken into account [26]
} 

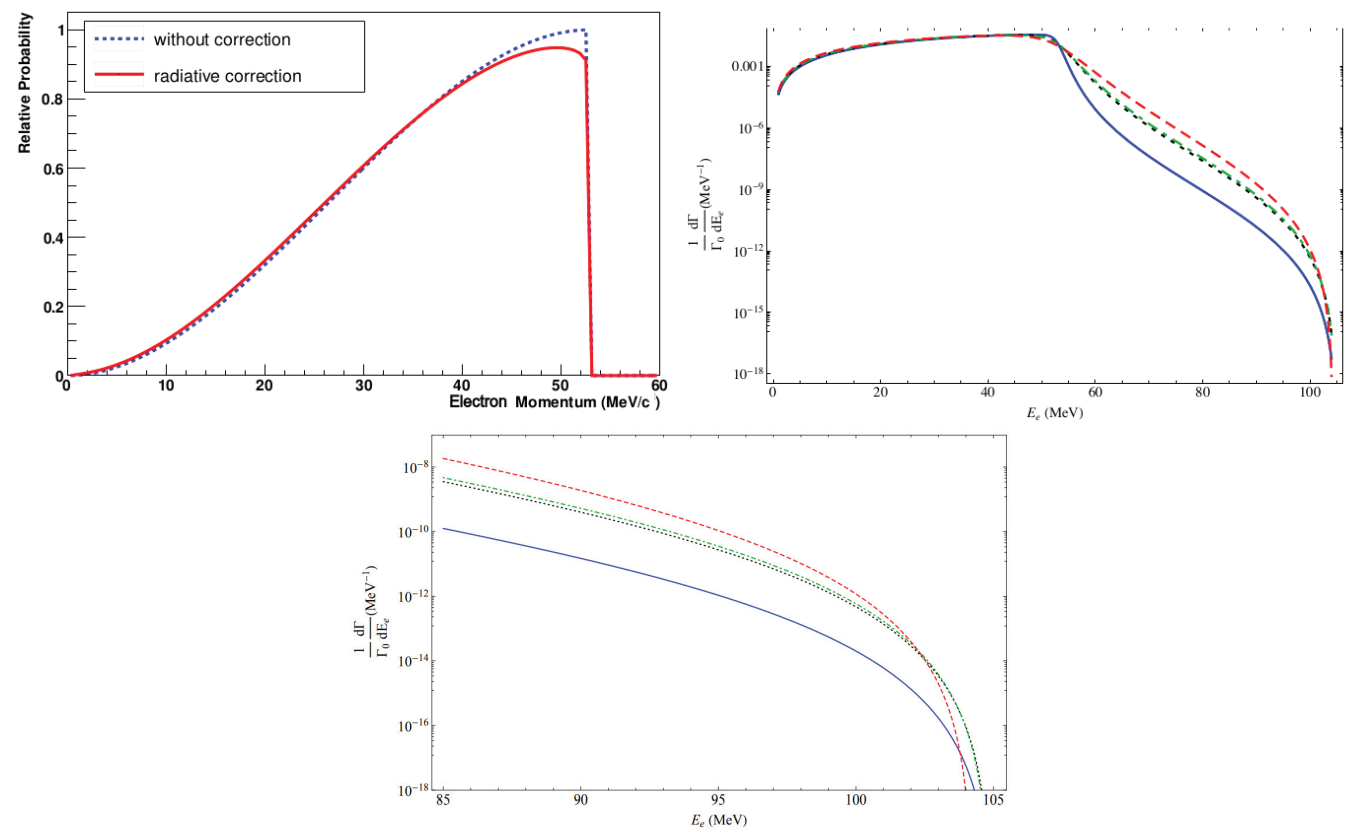

Figure 1.10: (Top Left) Michel spectrum of the outgoing electron in a muon decay in vacuum, shown with radiative correction and without. Both the curves show a kinematic endpoint at 52.8 MeV. Plots are from [12]. (Top Right) Outgoing electron differential spectrum in case of a muon decaying in the field of a nucleus, normalized to the free-muon decay rate $\Gamma_{0}$. The solid blue line is for carbon, the black dotted line for aluminum, the green dot-dashed line for silicon and the red dashed line for titanium. (Bottom) A zoom in the region near the endpoint. Plots and computation are from Czarnecki et al. [28]

a photon in the process. The maximum photon energy is just below the $m_{\pi}$ (so $\simeq 140 \mathrm{MeV}$ ) and a subsequent asymmetric pair production can fake a signal electron. This background can be faced in multiple ways: a degrader can be used to filter the beam from pions or a pulsed beam structure can be preferred in order to exploit the higher speed of this process with respect to captured muon decay time.

- Radiative Muon Captures (RMC)

This process is analogous to RPC but involves muons instead of pions. The spectrum is considerably softer and the photon does not have enough energy to produce electrons that can fake a signal CE. However, the RMC background can distort the DIO spectrum in a range $(80-100 \mathrm{MeV})$. Since the endpoint region of the DIO spectrum has never been measured, an extrapolation from lower energies is needed and, as a consequence, needs to be reliable.

\section{- Antiprotons}

Antiprotons can spiralize around the muon beam line and then hit the target producing $\pi^{0}$ and then photons and fake CEs. This background can be suppressed using a magnetic selectors or absorbers. It can also be completely eliminated using a proton beam energy below the threshold of antiproton production, that is $\sim 1.5 \mathrm{GeV}$.

The present limit for $\mu^{-} N \longrightarrow e^{-} N$ was set by the SINDRUM II experiment at PSI ([29]) in two steps. In 1993, SINDRUM II stopped $4.9 \times 10^{12}$ muons on a Titanium target and obtained a limit $R_{\mu e}<4 \times 10^{-12}$ (90\% CL) [23]; in 2006, it performed a new 
search using a gold target, with $4.4 \times 10^{13}$ stopped muons, decreasing the limit down to $R_{\mu e}<7 \times 10^{-13}(90 \% \mathrm{CL})[24]$.

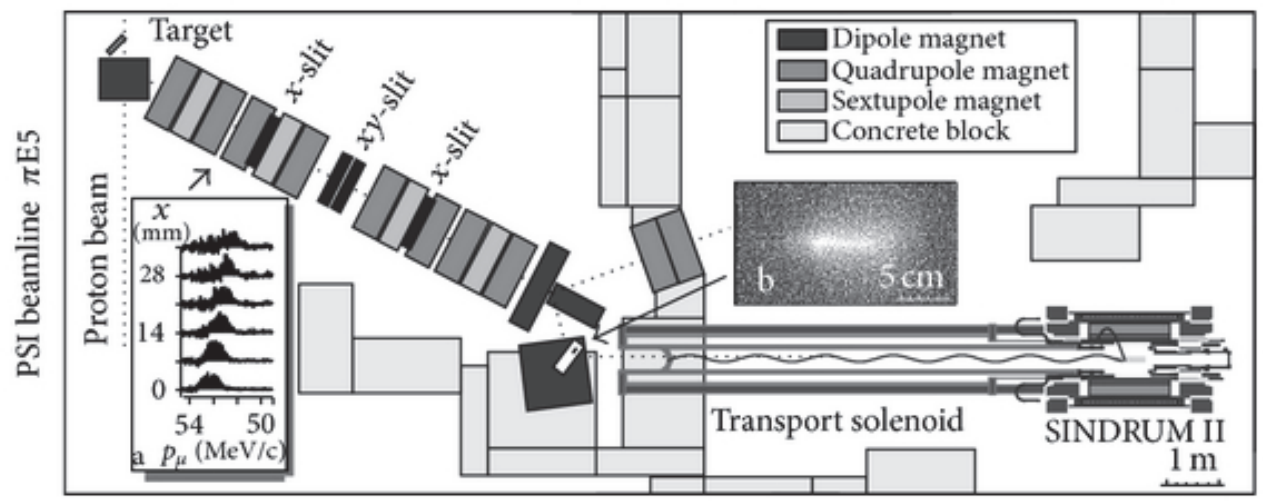

Figure 1.11: Schematic view of the SINDRUM II experiment. Inset a) shows the momentum dispersion measured at the position of the first slit system. The momentum distribution becomes wider when one side of the slit is increased. Inset b) shows a cross section of the beam (light dots) observed at the entrance of the transport solenoid.

The experimental design of SINDRUM II is shown in Fig. 1.11: a $590 \mathrm{MeV}$ (kinetic energy) continuous proton beam is extracted from the PSI ring cyclotron and hits a 40 $\mathrm{mm}$ carbon target (showed in the top left corner of Fig 1.11). Secondary $\mu, \pi$ and $e$ are produced from the interactions and transported to a degrader situated at the entrance of a transport solenoid. The degrader strongly reduces $\left(\sim 10^{-6}\right)$ the pion component without significantly affecting the muons that have a much longer range in the material. Negative muons are stopped in a solid target and the hypothetical conversion electron is detected by two drift chambers and a Cherenkov hodoscope.

The Mu2e experiment at Fermilab, that will be described in detail in the next chapter, aims to improve by a factor $10^{4}$ the SINDRUM II limit.

Mu2e direct competitors are the COMET and DeeMe experiments at J-PARK.

As it is illustrated in Fig. 1.12, COMET will operate in two different stages, called Phase I and Phase II. In Phase I a $8 \mathrm{GeV}$ (kinetic energy) proton beam will hit the production target; backward-going pions will decay into muons that will be transported through a bending solenoid to an Aluminum stopping target surrounded by a cylindrical drift chamber. In this first phase the experiment will focus on the evaluation of the different background contributions and will explore the new analysis techniques. COMET Phase II is very similar to Mu2e but it goes for a C-shaped muon beamline instead of an S-shaped (Mu2e) with the overall effect of a reduced beam intensity ( $\sim 30 \%$ less $)$ but a tighter momentum selection. In addition, the Phase II aluminum target will be followed by a curved spectrometer in order to select outgoing $\sim 100 \mathrm{MeV} / \mathrm{c}$ electrons. The electrons will be directed towards the detector, which consists of a straw tube tracker and a LYSO crystal electromagnetic calorimeter. Differently from Mu2e and COMET, DeeMe will use only one carbon target, both to produce and stop muons. A magnetic spectrometer and four multi-wire proportional chambers will be used to select signal electrons (Fig. 1.13). The simplicity of the detector is traded with a lower sensitivity goal of $5 \times 10^{-15}$. 

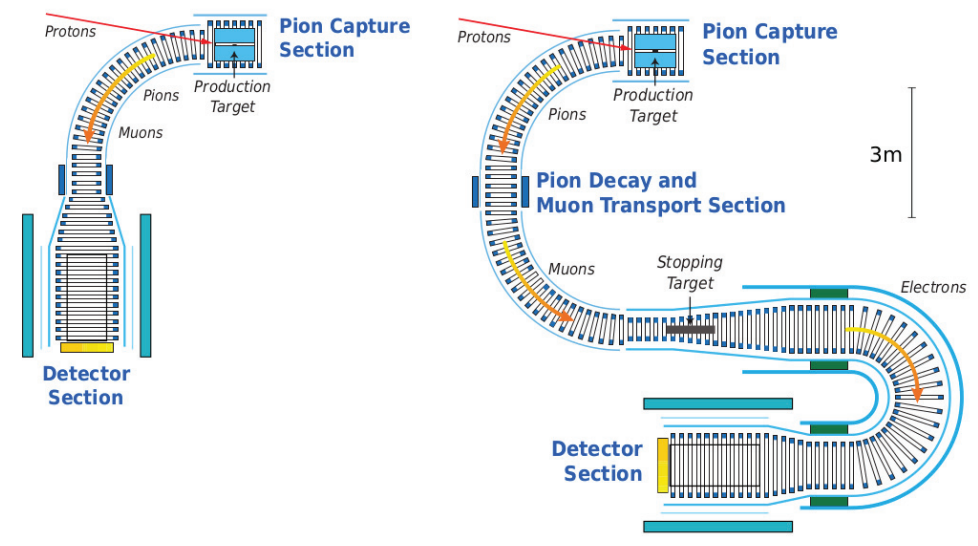

COMET Phase-I

COMET Phase-II

Figure 1.12: Schematic representation of the two phases of the COMET experiment.

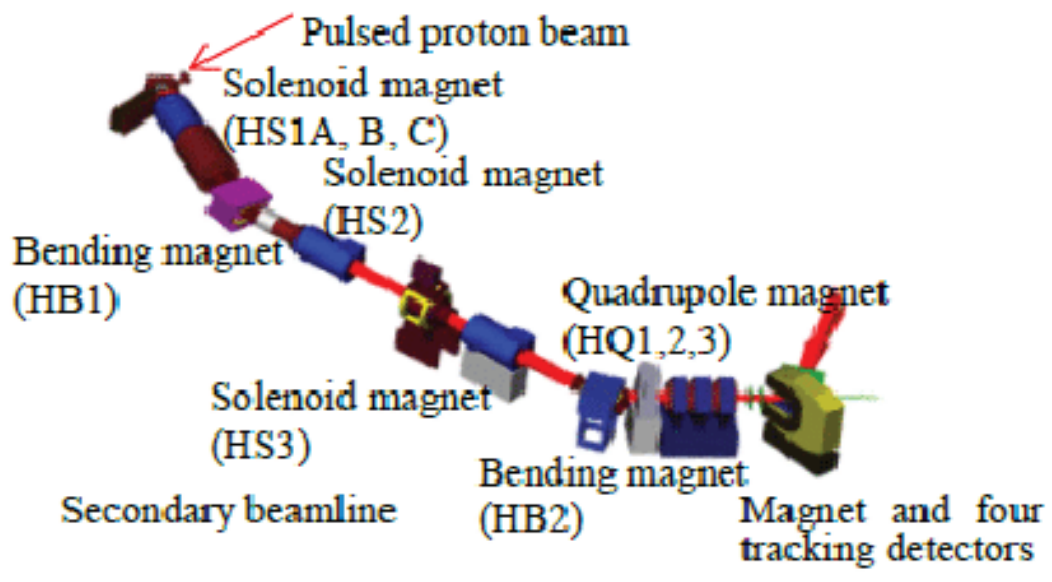

Figure 1.13: Schematic representation of main elements of DeeMe.

\subsubsection{The tau channel}

Before entering in a detailed discussion of the Mu2e experiment, it's worth to briefly present the status of CLFV search in the $\tau$ lepton sector. Thanks to its larger mass $\left(m_{\tau} \simeq 1777 \mathrm{MeV}\right)$ many flavour changing $\tau$ decays involving both leptons and hadrons can be investigated. Fig. 1.14 gives an idea of the abundance of the final states already studied. The tau sector of a CLFV search takes advantage from an higher predicted branching ratio compared to muons according to a power of the masses ratio $\left(\frac{m_{\tau}}{m_{\mu}}\right)^{\alpha}[12]^{8}$. However, the experimental search is affected by the short tau mean-life $\left(\tau_{\tau} \simeq 2.9 \times 10^{-13}\right)$, that makes impossible to form tau beams, and by the lower production multiplicity in hadrons decay. These two factors overcome the gain in branching ratio, but not excessively, making this channel a competitive alternative to muons.

The current best limits have been set by the BaBar and Belle experiments. Both exploit the full power of an accelerator complex (PEP-II collider at SLAC for BaBar

\footnotetext{
${ }^{8} \alpha$ depends on the underlying model.
} 


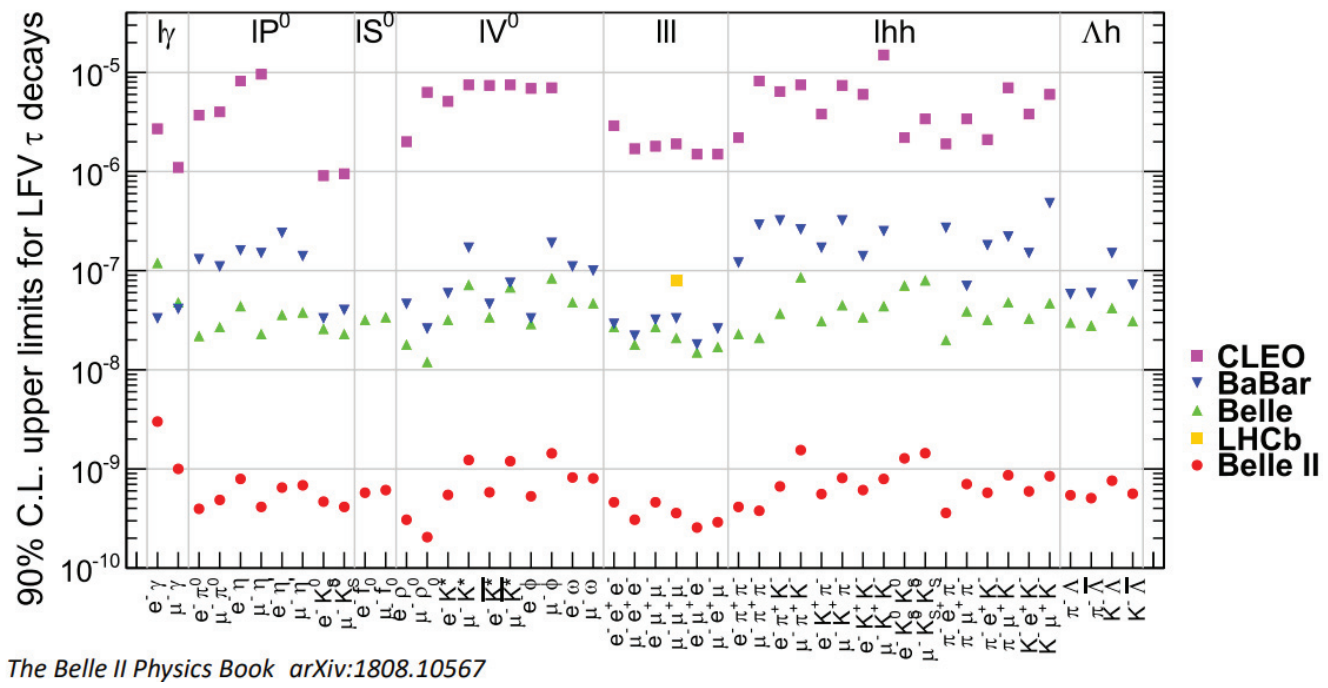

Figure 1.14: 90\% CL upper limits on CLFV tau decays. The green cones represent the best limits obtained by the Belle experiment. Babar (blue cones) still holds the best results for the $\tau \rightarrow e \gamma$ and $\tau \rightarrow \mu \gamma$ channels. Belle 2 expected results for the $50 a b^{-1}$ run are shown in red.

and KEKB for Belle) and detectors with an excellent particle identification and tracking capability. In those experiments a $\tau$ pair originates from the decay of the $\Upsilon(4 s)$ resonance at $\sqrt{s}=10.58 \mathrm{GeV}$, whose branching ratio in $\tau^{+} \tau^{-}$is comparable to the one in $b \bar{b}$. Table 1.2 reports the current limits set on the most promising tau channels.

\begin{tabular}{ccc} 
Process & Upper Limit $(90 \%$ CL) & Reference \\
\hline$\tau \rightarrow e \gamma$ & $<3.3 \times 10^{-8}$ & BaBar [30] \\
$\tau \rightarrow \mu \gamma$ & $<4.4 \times 10^{-8}$ & BaBar [30] \\
$\tau^{-} \rightarrow e^{-} e^{+} e^{-}$ & $<3.6 \times 10^{-8}$ & Belle [31] \\
$\tau^{-} \rightarrow \mu^{-} \mu^{+} \mu^{-}$ & $<3.2 \times 10^{-8}$ & Belle [31] \\
$\tau^{-} \rightarrow e^{-} \mu^{+} \mu^{-}$ & $<4.1 \times 10^{-8}$ & Belle [31] \\
$\tau^{-} \rightarrow e^{+} \mu^{-} \mu^{-}$ & $<2.3 \times 10^{-8}$ & Belle [31] \\
$\tau^{-} \rightarrow \mu^{-} e^{+} e^{-}$ & $<2.7 \times 10^{-8}$ & Belle [31] \\
$\tau^{-} \rightarrow \mu^{+} e^{-} e^{-}$ & $<2.0 \times 10^{-8}$ & Belle [31]
\end{tabular}

Table 1.2: Current 90\% CL upper limits on the most sensitive CLFV tauonic channels.

In the present days, the Belle 2 collaboration [32] is working on an improvement of these limits. With the integrated luminosity of $50 \mathrm{fb}^{-1}$ acquired during the last run, a sensitivity comparable to the best actual limits is expected. In the future (not before 2030) the experiment will take advantage from an integrated luminosity of $50 a b^{-1}$ [33] and is expected to improve the current limits down to $2 \times 10^{-10}$ for the background-free channel $\tau \rightarrow 3 \ell$, and $2 \times 10^{-9}$ for $\tau \rightarrow \mu \gamma$. The other expectations of the $50 a b^{-1}$ run at Belle 2 are shown in Fig 1.14 as red markers. 


\section{Chapter 2}

\section{The Mu2e experiment}

The Mu2e experiment will search for the charged-lepton flavour violating process

$$
\mu^{-} \mathrm{Al} \longrightarrow e^{-} \mathrm{Al}
$$

where the muon converts into a $104.97 \mathrm{MeV} / \mathrm{c}$ electron in the field of an Aluminum nucleus. Muons stopped in the aluminum target will be captured by the atoms and reach the ground state in less than 1 ps. Muonic Al has a lifetime of 864 ns and can disappear through:

- a muon decay in orbit (DIO, 39\%): $\mu^{-27} \mathrm{Al} \rightarrow e^{-} \bar{\nu}_{e} \nu_{\mu}{ }^{27} \mathrm{Al}$

- a nuclear muon capture (61\%): $\mu^{-27} \mathrm{Al} \rightarrow \nu_{\mu}{ }^{27} \mathrm{Mg}^{*}$

- a muon to electron conversion $\left(<10^{-13}\right): \mu^{-27} \mathrm{Al} \rightarrow e^{-27} \mathrm{Al}$

This chapter will describe the Mu2e experiment design focusing on its peculiarities that are relevant for the identification of the conversion electrons against the main possible backgrounds.

\subsection{Experimental layout: magnets and detectors}

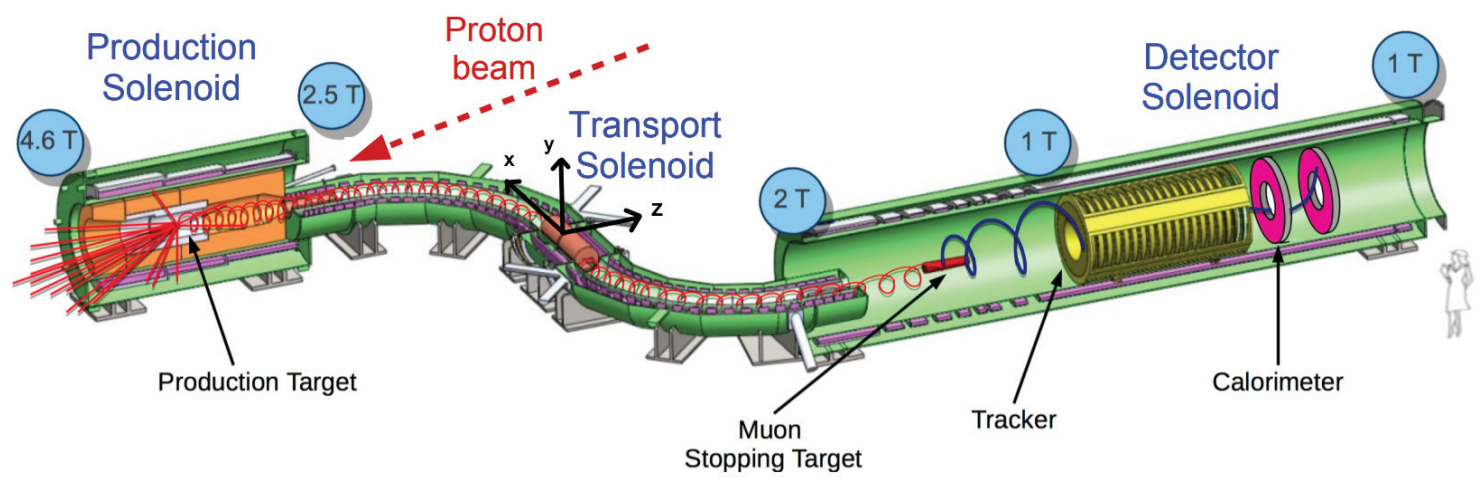

Figure 2.1: Scheme of the Mu2e experiment with an highlight on spiraling muons (red) and CEs (blue) trajectories. Light blue circles represent indicative values of the magnetic field in each region. The Mu2e coordinate system origin is also shown. The Cosmic Ray Veto, covering the complete Detector Solenoid and half of the Transport Solenoid is not drawn here. 
A schematic view of the experiment is shown in Fig. 2.1. The main structure consists of three solenoids: the Production (PS), the Transport (TS) and the Detector Solenoid (DS). In Mu2e, a $8 \mathrm{GeV}$ kinetic energy proton beam hits a tungsten target inside the Production solenoid. Many particles are produced from these interactions but the magnetic field is specifically designed to redirect pions, kaons and muons produced in their decays to the Transport Solenoid. This S-shaped magnet allows only the particles with the wanted charge and momentum to reach the Detector Solenoid where the aluminum target is located and muons can possibly stop and convert. The DS hosts the straw tube tracker and two CsI calorimeter disks providing measurement of event kinematics. A precise low-rate Germanium detector and a LaBr crystal allowing the measurement of muon captures is located downstream of the DS, out of scale on the right of Fig. 2.1. A system of scintillators, called the Cosmic Ray Veto (CRV), covers the complete detector area and extends up to the half of TS vetoing every event within a time coincidence with a cosmic rays. An extinction monitor, downstream of the proton beam (out of scale on the left of Fig. 2.1) is used to monitor the presence of out of time protons that can introduce additional background.

The general coordinate system is a Cartesian frame centered in the middle point of TS: the $\mathrm{z}$-axis is parallel to the central axis of PS and DS (PS is negative $\mathrm{z}$ and DS is positive $\mathrm{z}$ ), the $\mathrm{y}$-axis points upwards and the $\mathrm{x}$-axis closes a right-handed triad.

\subsection{The proton beam line}

The Mu2e proton beam will be provided by the Fermilab accelerators system shown in Fig. 2.2 . The system will be shared with $\mathrm{NO} \nu \mathrm{A}$ (NuMI Off-axis $\nu_{e}$ Appearance, [34]) experiment so that only part of the running time will be dedicated to Mu2e. The beam sharing with the Fermilab neutrino program will also have an important impact on the Mu2e schedule: given the planned accelerator upgrade for the long baseline neutrino program there will be no beam since summer 2025 for about 2 years. For this reason Mu2e has opted for a 2 phases run plan: a first run will take place in 2024 and 2025, before the shutdown, will use half of the proton beam intensity and will need a reduced concrete shielding, a second run, after the shutdown, will use the full beam intensity and a stronger (and more expensive) concrete shielding.

Proton beam formation can be summarized as follows:

1. First of all, a Cockcroft-Walton generator accelerates negative hydrogen ions up to a kinetic energy of $750 \mathrm{keV}$.

2. In a LINAC these ions are accelerated up to $400 \mathrm{MeV}$ and electrons are removed.

3. Then protons are injected into the Fermilab Booster Synchrotron Ring where they are accelerated up to $8 \mathrm{GeV}$ and organized into $1.7 \mu$ s long packages called "batches".

4. By the time Mu2e is ready for beam, the Booster will be capable of delivering proton batches of $\approx 4 \times 10^{12}$ protons to the following Recycler Ring $(\mathrm{RR})$ at a rate of $15 \mathrm{~Hz}$ (one proton batch every $67 \mathrm{msec}$, this cycle time is usually denoted as "tick"). The accelerator cycle in the Recycler Ring is organized around a 1333 ms (20 ticks) "Main Injector Ramp". This term refers to the sequence of magnet and radio-frequency system ramps that accelerate $\mathrm{NO} \nu \mathrm{A}$ designated protons from $8 \mathrm{GeV}$ to $120 \mathrm{GeV}$ kinetic energy. The $\mathrm{NO} \nu \mathrm{A}$ experiment requires 12 proton ticks 


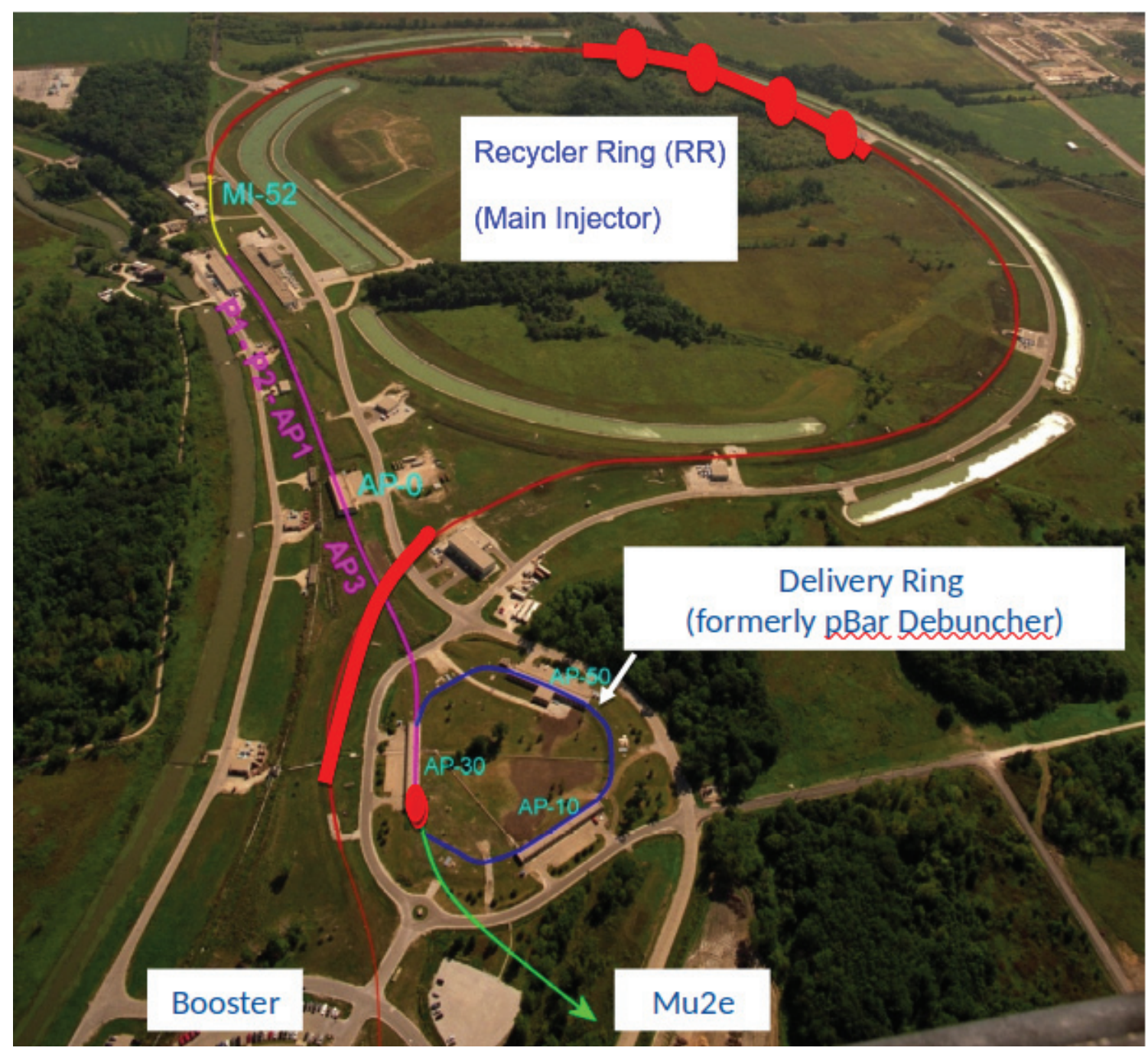

Figure 2.2: Mu2e proton delivery for the low-intensity, i.e. 1 batch, configuration. Booster "batches" are here highlighted as thick red lines. One batch is injected for Mu2e every RR cycle (1333 ms), and divided into 4 bunches of $10^{12}$ protons each (red bulks). The bunches are extracted one at a time to the Delivery Ring and inside it, as the bunch circulates, it is resonantly extracted to produce the desired beam structure.

to meet its requirements. In a 20 tick cycle, this leaves 8 injection slots available for Mu2e use before the Main Injector ramp is initiated again for the next cycle. Within these, the first and an half tick will be used to inject one batch and operate a manipulation on the beam. Here the batch is reorganized into four smaller structures of $\approx 1 \times 10^{12}$ protons each, called "bunches", and a $10^{-5}$ fraction of out of bunch protons is naturally achieved. The rest is left for the next step transportation. The 20 tick scenario is illustrated in Figure 2.3 [35].

5. These four bunches are individually transferred from the Recycler Ring to the Delivery Ring (DR) through a series of connected beam-lines. These transfers ("spills") occur at $112.3 \mathrm{~ms}$ intervals.

6. As shown in Fig. 2.4, each bunch in the DR is now slow spilled continuously for the 


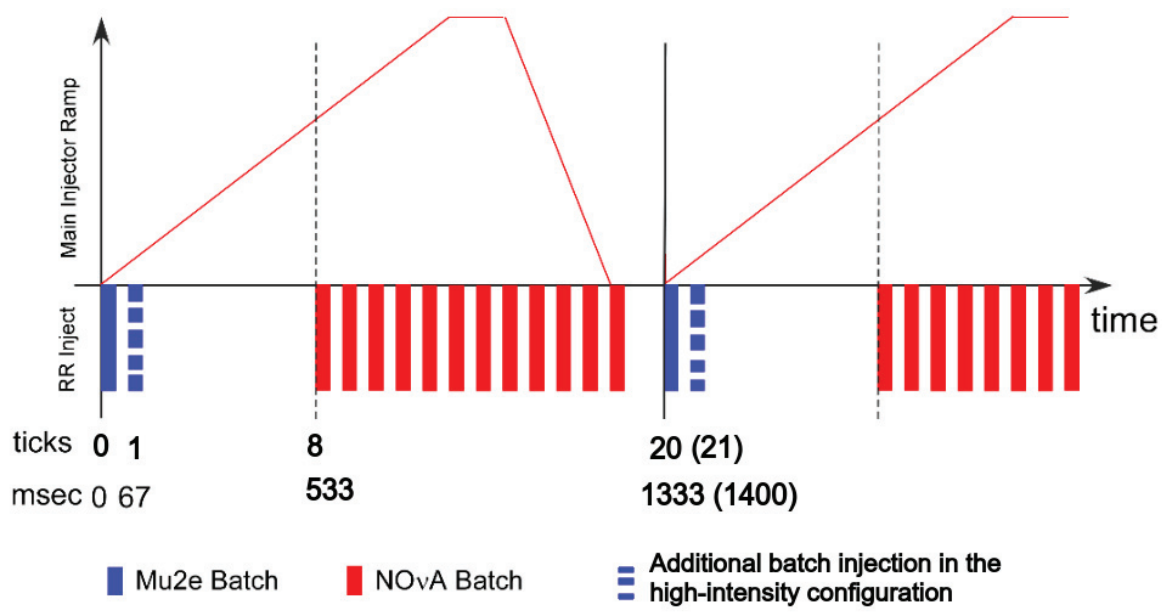

Figure 2.3: Accelerator timeline distribution between $\mathrm{NO} \nu \mathrm{A}$ and Mu2e. The blue and red bars mark the injections of designated proton batches into the RR. The red curve is the "Main Injector ramp" described in the text. The total length of the cycle shown here is 20 ticks $=1.333 \mathrm{~s}$. In the full-intensity configuration one more bunch will be injected resulting in a 21 ticks structure.

entire time needed before the next bunch arrival ${ }^{1}$. In order to obtain the desired beam structure a proton pulse ${ }^{2}$ with $1.6 \times 10^{7}$ protons must be resonantly extracted every $1.695 \mu \mathrm{s}$, and so must be the DR revolution period. Within the $107.3 \mathrm{~ms}$ the DR is thus able to resonant extract 63298 proton pulses from a single bunch to the Production Target. (i.e. $107.3 \mathrm{~ms}=63298$ pulses $\times 1.695 \mu \mathrm{s} /$ pulse).
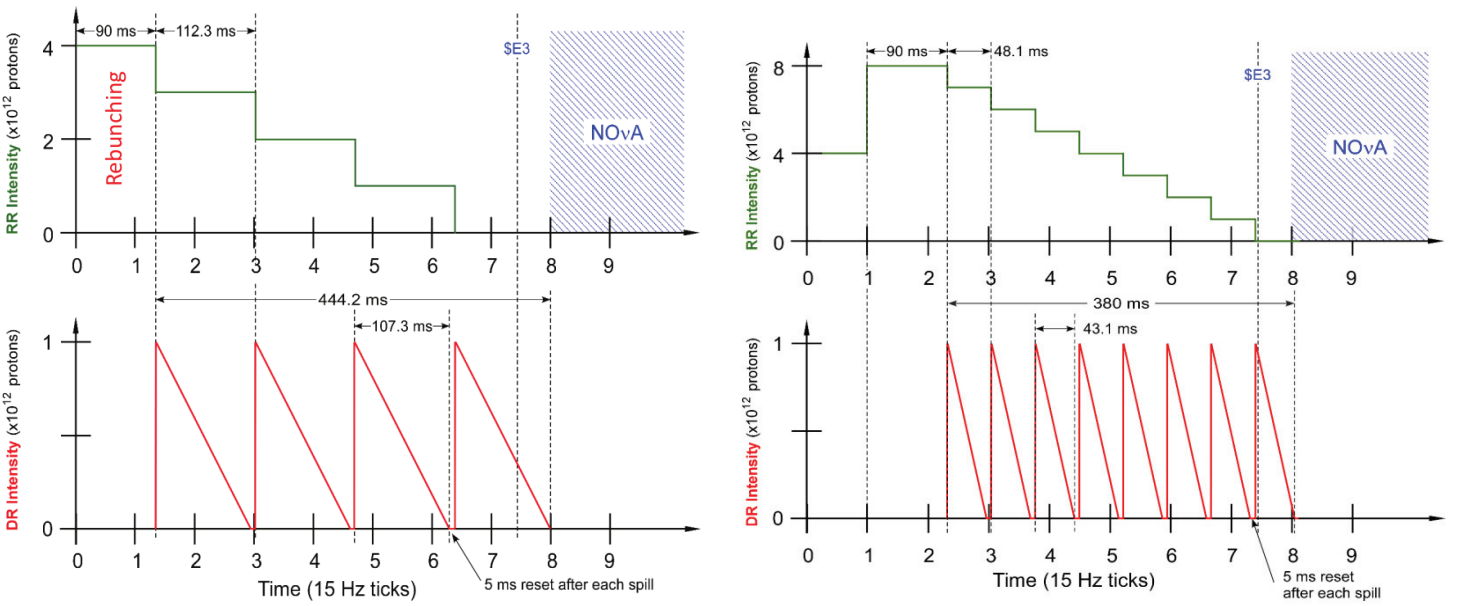

Figure 2.4: Mu2e beam manipulation for low-intensity (LEFT) and full-intensity (RIGHT) configuration. The green graph shows the Recycler Ring beam intensity as a function of time. The red plot shows the Delivery Ring beam intensity as a function of time.

In the later full-intensity phase (right plot of Fig. 2.4), one additional tick will be used to inject a total of two batches in the Recycler Ring while the rest will be left to the same

\footnotetext{
${ }^{1} 5 \mathrm{~ms}$ are left for the Delivery Ring reset after each spill.

${ }^{2} \mathrm{~A}$ pulse (also referred to as a beam pulse or proton pulse) is the beam resonantly extracted from the Delivery Ring to the Mu2e proton target in a single revolution period.
} 
rearrangement but into eight bunches. The $\mathrm{RR}$ cycle is also rearranged into a $1.4 \mathrm{~s}(21$ ticks) period. This imply an extraction every $48.1 \mathrm{~ms}$ and 25440 proton pulses spilled per bunch. The full-intensity proton density is $3.9 \times 10^{7}$ protons/pulse, a factor $\sim 2.4$ more than the one scheduled for the first 2 years run.

\subsubsection{Beam intensity profile}

In spite of the efforts of the accelerator experts, the proton beam intensity cannot be constant. A precise knowledge of the proton intensity distribution is required in order to have a reliable prediction of the detectors occupancy and of the trigger maximum bandwidth. In absence of experimental data able to validate a model of Mu2e slow extraction, the intensity fluctuation is parameterized as a log-normal distribution that well fits the performances of other existing facilities.

Slow extraction beam intensity distribution is often characterized in terms of the "Spill Duty Factor" (SDF), which is a measure of relative spread of the proton intensity distribution:

$$
S D F=\frac{1}{1+\left(\frac{R M S}{I_{\text {avg }}}\right)^{2}}
$$

with "RMS" and $I_{\text {avg }}$ representing respectively the Root-mean-square deviation and the mean value of the distribution. In order to meet the technical design requirements, in particular the dead time induced by the Cosmic Ray Veto system, Mu2e will need a $\mathrm{SDF}>60 \%$ [36] (Fig. 2.5). This is different from the original requirement set in the Mu2e Technical Design Report (TDR, [37]): $0.5<\frac{I}{I_{\text {avg }}}<1.5$, which was found to be unrealistic (it is observed that resonant extraction usually gives high intensity tails) and poorly-specified (a minimum intensity requirement is not needed and is not necessary that all pulses meet the requirement).

\subsubsection{Mu2e beam structure}

The design of the proton beam structure for Mu2e has taken into account two main considerations:

- Proton collisions in the Production Target can make neutral pions, which immediately decay into photons (with an half-life of $8.4 \times 10^{-17} \mathrm{~s}$ ). Photons can finally convert into electrons and create a "flash" of particles that potentially overwhelms the detectors for a few hundred ns.

- Proton collisions also make charged pions. The negative component is supposed to decay into muons and form the muon beam. If such pions reach the Stopping Target before they decay, they can be bound in pionic atoms and then go trough nuclear capture with the emission of a $\lesssim 140 \mathrm{MeV}\left(m_{\pi}\right)$ photon $\left(\pi^{-} N \rightarrow \gamma N^{\prime}\right)$. An asymmetric conversion of the photon can finally lead to a $\sim 105 \mathrm{MeV}$ electron. This process, called Radiative Pion Capture (RPC), can potentially be a serious source of background for a muon conversion experiment.

The two mentioned processes scale with muon beam intensity and represent both a technical and physical limit for the experiment. First, an increased beam intensity leads to more 


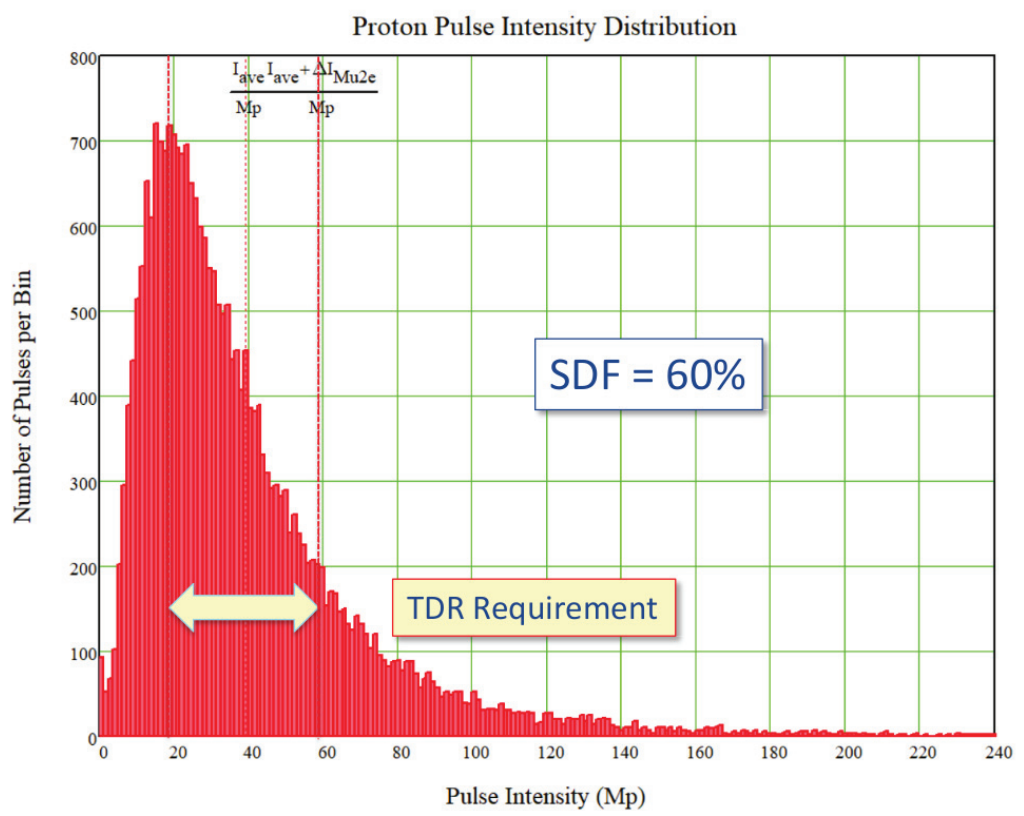

Figure 2.5: Proton pulse intensity distribution corresponding to a SDF of $60 \%$, obtained from a MC simulation. Scale horizontal axis by $1 / 2.4$ to get distribution for reduced intensity running.

radiation damages from the flash and decreases the lifetime of the detector elements. Secondly, the RPC background is important for the choice of the muon stopping target. Mu2e has chosen the Aluminum whose muonic atom lifetime (864 ns) well matches the inter-bunch period. In principle one could think to use nuclei with high $\mathrm{Z}$ to have a shorter muonic atom lifetime and collect a larger fraction of muonic atom decays before the next bunch with its flash arrives, but, doing so, we will find our start of the time window (and the majority of the signal electrons) with only a small delay with respect to the pion arrival. This leading to a larger inefficiency for muonic atoms with a shorter lifetime. The above considerations, together with the fact that the radiative muon capture (RMC) is well separated in energy from the signal has brought to the choice of the Al as optimal material for the stopping target. ${ }^{3}$

Fig. 2.6 shows the Mu2e proton bunch time structure with the arrival time on the stopping target for pions and muons and a possible choice for the analysis window used for the conversion electrons search. At full (reduced) intensity, bunches with $\simeq 3.9 \times 10^{7}$ $\left(\simeq 1.6 \times 10^{7}\right), 8 \mathrm{GeV}$ kinetic energy protons will arrive every $1695 \mathrm{~ns}$. The arrival time distribution for pions at the detector solenoid allows a measurement window to start when $\mathrm{RPC}$ component is extinguished within an acceptable level (an $\mathrm{O}\left(10^{11}\right)$ suppression) and muons are still decaying. The data acquisition will start as early as about $700 \mathrm{~ns}$ after the arrival of the proton bunch on the production target. The analysis window should be optimized according to the beam intensity aiming to reach the best sensitivity (Chap. 5).

It's important to noise that RPC and beam flash suppression strictly relies on the proton bunch time structure: in order to keep the RPC background under control the fraction of protons out of time with respect to the bunch (called "extinction factor"), must be kept below $10^{-10}([37])$ and continuously monitored.

\footnotetext{
${ }^{3}$ Other materials, such as the Titanium, will be considered for the second phase of Mu2e, also according to the results of the first phase.
} 


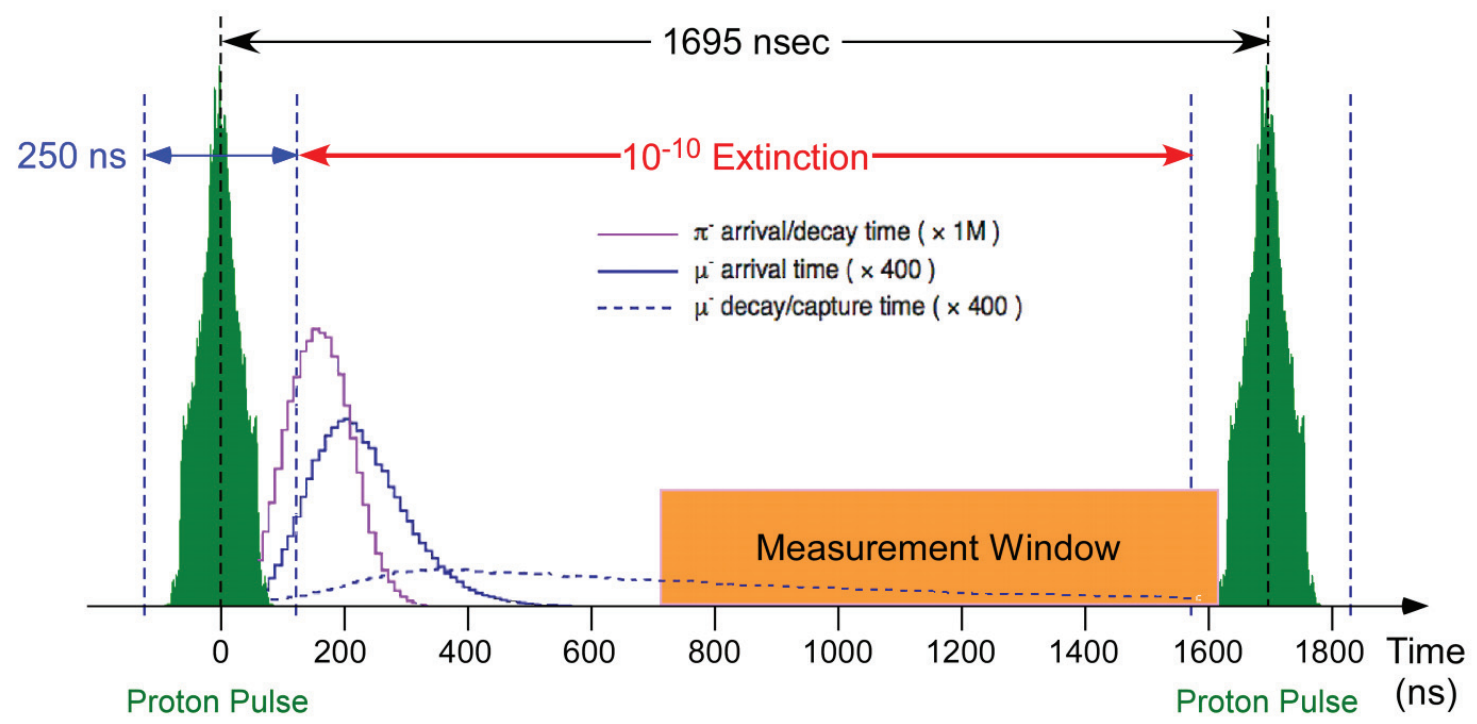

Figure 2.6: The Mu2e beam timing at full-intensity. A pulse with $\approx 3.9 \times 10^{7}, 8 \mathrm{GeV}$ kinetic energy protons arrives every $1695 \mathrm{~ns}$. The arrival time distribution for pions that arrive at the detector solenoid (greatly reduced by the Mu2e Solenoid system) is shown, along with the arrival time for muons and the decay or capture time (both with an $864 \mathrm{~ns}$ lifetime). The "Measurement window" is the period of time for which Mu2e will analyze data. The pulse shape is more complicated than the idealized form shown here.

\subsubsection{Extinction System}

At the moment protons leave the Delivery Ring, a $10^{-5}$ extinction is already provided.

A dedicated system designed to further remove out-of-time protons and meets the project requirement of $10^{-10}$, resides in the beamline connecting the Delivery Ring to the Mu2e Production Solenoid: it consists of three horizontal collimators and a pair of horizontally bending fast dipoles. The extinction is accomplished by requiring the fast (AC) dipoles to be synchronized to the beam in such a way that out-of-time beam will see a deflecting field while in-time beam will pass through the system unmolested. The actual design and simulation work make it possible to reach an extinction level which is $<1.6 \times 10^{-12}$ and a transmission efficiency for in-time beam of 99.5\% [38] .

In Fig. 2.7 proton time distribution before and after passage through the beamline extinction section are shown. Much further details on the beam longitudinal structure and extinction can be found in [39].

\subsubsection{The Extinction Monitors}

Mu2e will measure the proton extinction factor in two ways:

1. A fast upstream monitor before the AC dipole, designed to detect early problems in the beam.

2. A "telescope" placed downstream of the PS looking for protons scattered off the production target.

The telescope is schematized in Fig. 2.8. A small window slightly off beam axis allows protons scattered by the Production Target to reach a momentum-selecting filter consisting 


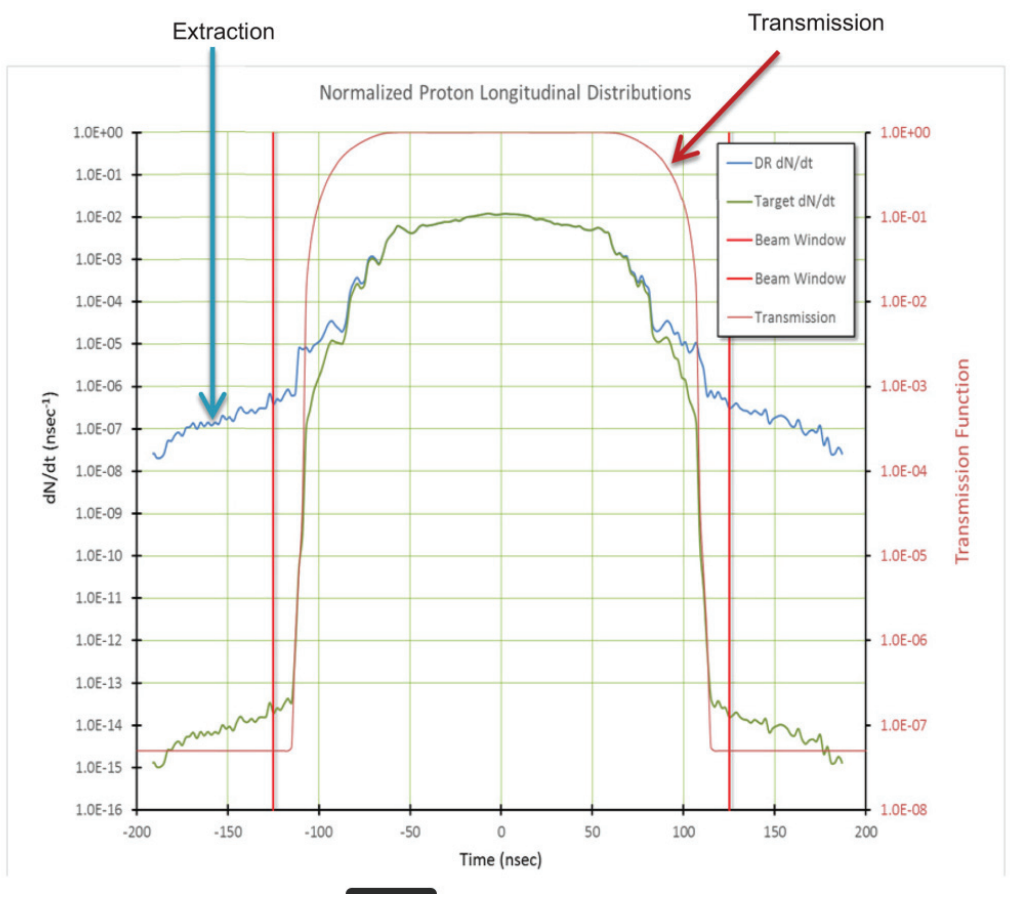

Figure 2.7: Proton time distribution ( $\mathrm{dN} / \mathrm{dt}$ ) before (blue) and after (green) passage through the beamline extinction section based on a simulation. The transmission function of the extinction system is plotted in red.

of collimators and a permanent dipole magnet selecting $4.2 \mathrm{GeV} / \mathrm{c}$ particles. Two sensor stacks with four pixel planes each and a permanent magnet in-between allow to reconstruct the particle momentum.

A possible background originates from muons produced by spurious interactions in the heat and radiation shield in the Production Solenoid: a dedicated detector is therefore installed behind the spectrometer to establish the muon content of both in-time and out-of-time beams. To be identified as a muon, the candidate particle must trigger a first scintillator plane, survive a series of steel plates and hit one of the following three scintillators, with more material in between (right side Fig.2.8).

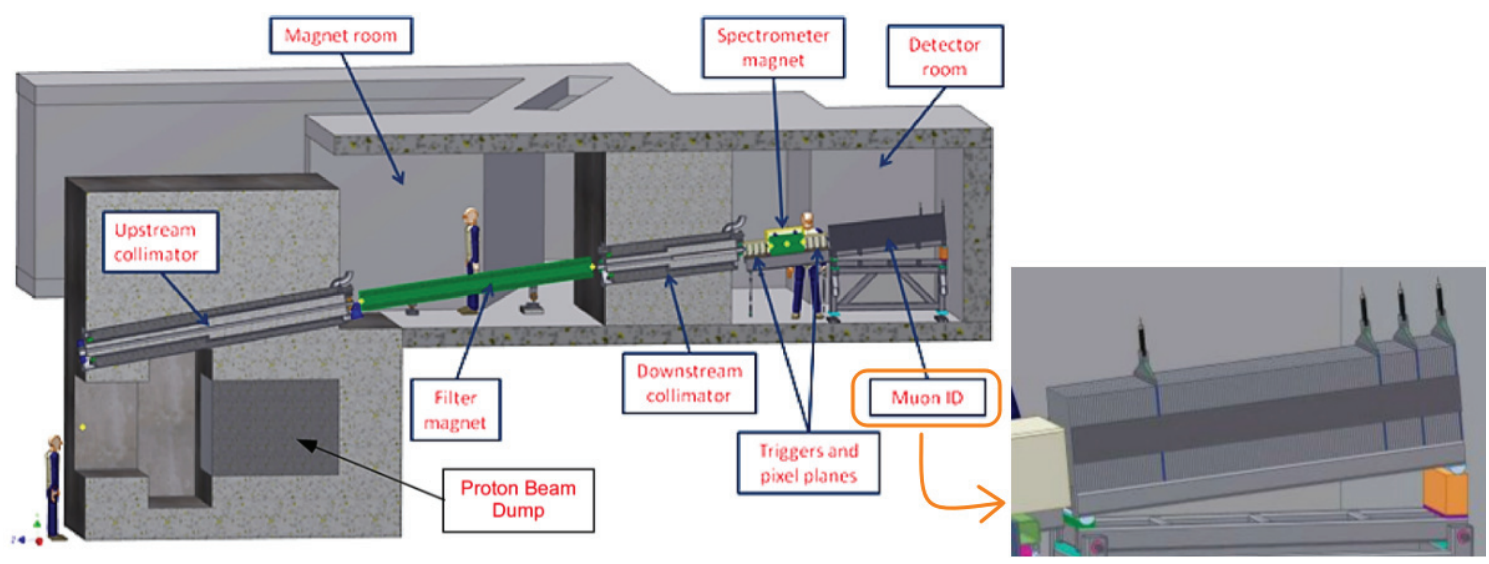

Figure 2.8: Representation of the extinction monitor with a closer look at the Muon ID system.

Given the very small number of expected out of bunch protons, the telescope will need 
few hours to achieve a measurement with a sensitive statistical error ${ }^{4}$, this, in any case, will allow to detect problems before a significant amount of data is lost.

\subsubsection{Production Target (PT)}

Protons from the Delivery Ring enter the Production Solenoid at $14^{\circ}$ with respect to the z-axis and hit the Mu2e Production Target (PT) which is responsible for stopping protons and producing pions. The physical requirement is then an optimal stopping power for protons with a minimal pions energy loss.
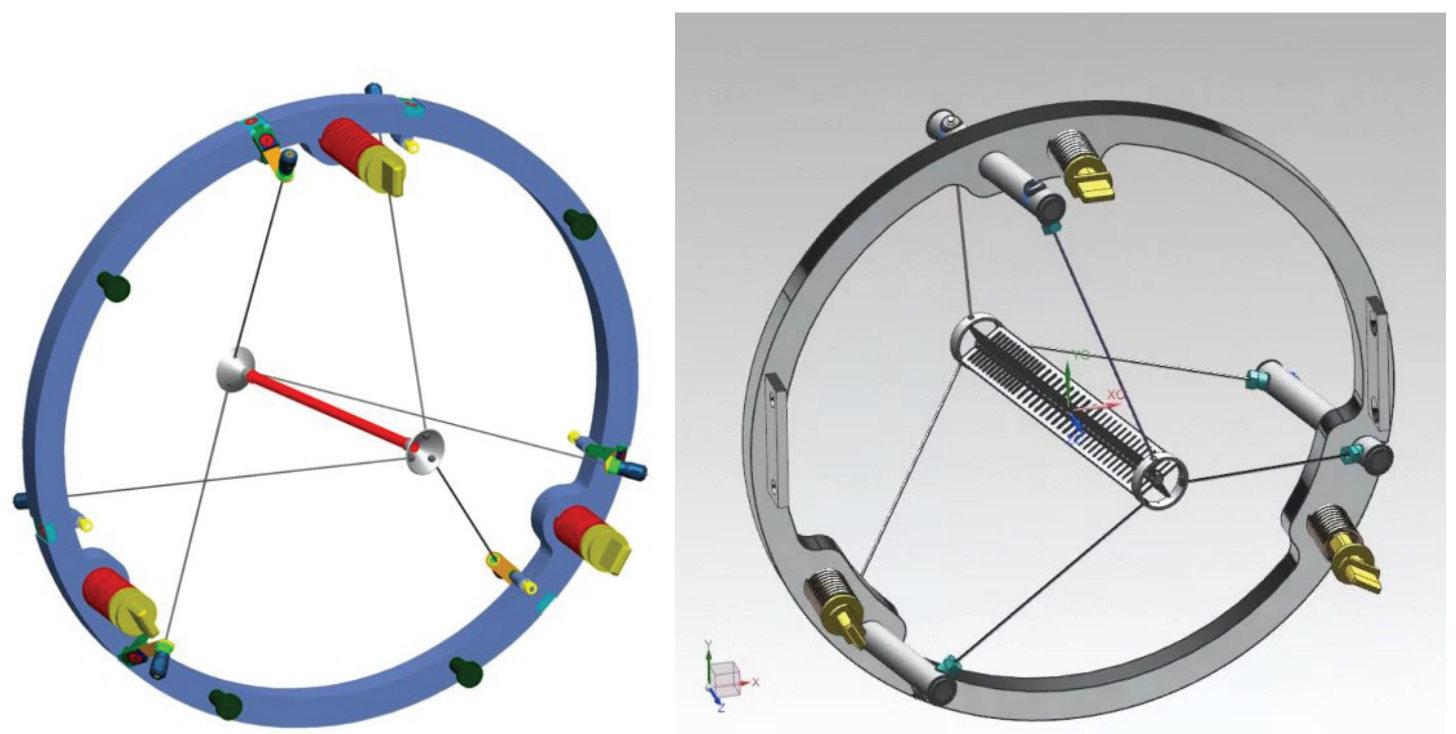

Figure 2.9: The two versions of the Mu2e production target: original project (LEFT) and current design (RIGHT). Both are suspended by spokes connecting to a bicycle wheel structure.

In the original project $([37])$, the Production Target consisted in a tungsten $(\mathrm{A}=$ 183.84) rod, $160 \mathrm{~mm}$ long with a 6.3 diameter (left side of Fig. 2.9). Because of the high melting point $\left(3422{ }^{\circ} \mathrm{C}\right)$, the refractory tungsten is ideal: it is also resistant to wear, corrosion and deformation, finally the high atomic number ensures a high rate of beam-target interactions.

Nevertheless, some recent tests has demonstrated that such a target needs some adjustments to resist stresses and radiation damage [40]. Some of the encountered problems are related to the location where the target is host: vacuum is inefficient for cooling; the presence of $\mathrm{O}_{2}$ impurities promotes chemical erosion; radiation damage causes heat injuries and mechanical creep.

The current version (called "Hayman 2"), showed in the right side of Fig. 2.9, has circular rings at the end and its core is finned and segmented to allow for sag minimization and temperature control. This definitive structure allows the target to resist for more than 1 year of operation but with a cost in muon yield from 0.0019 (TDR value) to $\sim 0.0016$ stopped muons / Proton on Target [41].

\footnotetext{
${ }^{4}$ This integration time depends on the final achieved extinction and the Duty Factor (defined as the Total Spill Time $\div$ Main Injector Cycle Length $=(8 \times 48.1 \mathrm{~ms}-7 \times 5 \mathrm{~ms}) \div 1400 \mathrm{~ms}=24.6 \%$
} 


\subsection{The muon beam line}

\subsubsection{Production Solenoid (PS)}

First in the chain of Mu2e magnets is the Production Solenoid (PS) (2.10, left). The PS is $4 \mathrm{~m}$ long and, as the other two following magnets, made of NbTi superconducting cables stabilized with aluminum and cooled with a liquid helium system. To avoid damages to the superconducting materials the PS is internally covered with a heat and radiation shield.

The role of PS is to collect and focus $\pi^{-}, K^{-}$and subsequent $\mu^{-}$leaving the Production Target to the Transport Solenoid by supplying a graded magnetic field of $\sim 1 \mathrm{~T} / \mathrm{m}$. As shown in right side of Fig. 2.10, the field increases from the end of the PS to a maximum of $4.6 \mathrm{~T}$, then it monotonically decreases (with no more than $\pm 5 \%$ non-linearity) to $2.5 \mathrm{~T}$ over the remaining length of $2.8 \mathrm{~m}$.
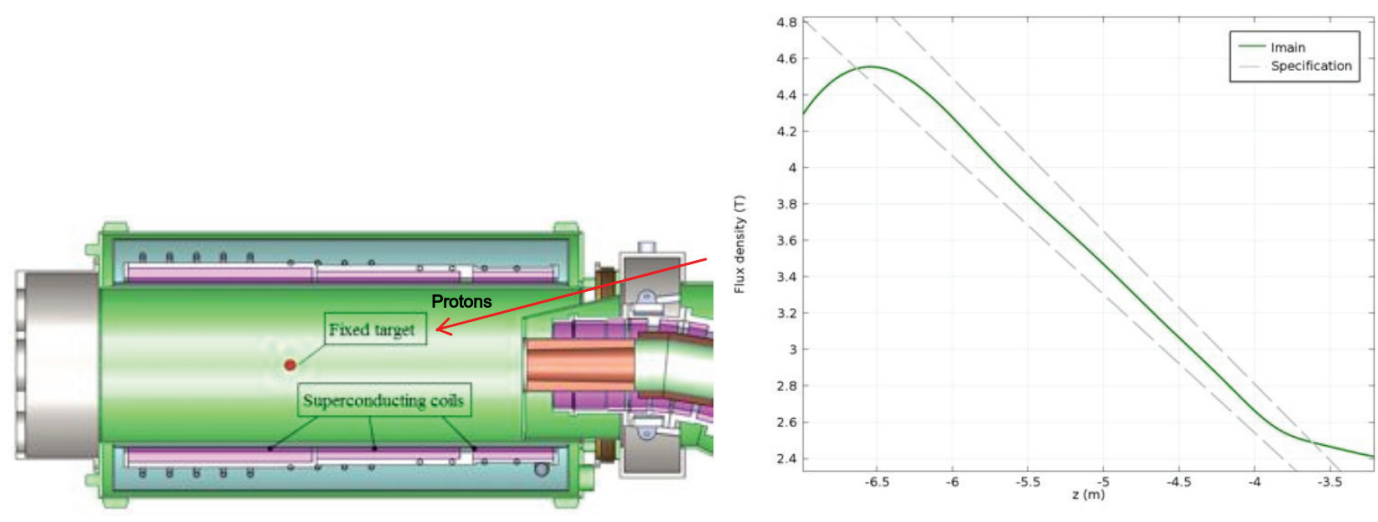

Figure 2.10: (LEFT) Representation of the Production Solenoid internal structure. The incoming proton direction is highlighted with a red arrow. (RIGHT) Magnetic field dependency from the z-coordinate inside the Production Solenoid.

Any positive gradients have to be carefully understood since it can create the dangerous effect of "particle trapping". Trapped pions or muons take much more time to exit the PS and can elude the timing suppression provided by the analysis window.

The "backward" scheme, with the proton beam pointing to the opposite side with respect to the TS entrance, is the result of a compromise between optimizing the stopped muon rate and avoiding the detector to be overwhelmed by an excessive flux of particles.

The total muon collection efficiency receives contribution from backward produced particles and some forward going that are reflected in the magnetic field or scattered back inside the PT.

\subsubsection{Transport Solenoid (TS)}

The Transport Solenoid (Fig. 2.11) is an "S-shaped" pipe filled with collimators and a magnetic field specifically designed to maximize the transport of negative muons that stop in the Stopping Target and strongly suppress all other particles. It is divided into 5 regions:

- A first straight part (TS1), connected to the Production Solenoid, is necessary to optimize the beam transmission. It also holds the first collimator (COL1), a 15 


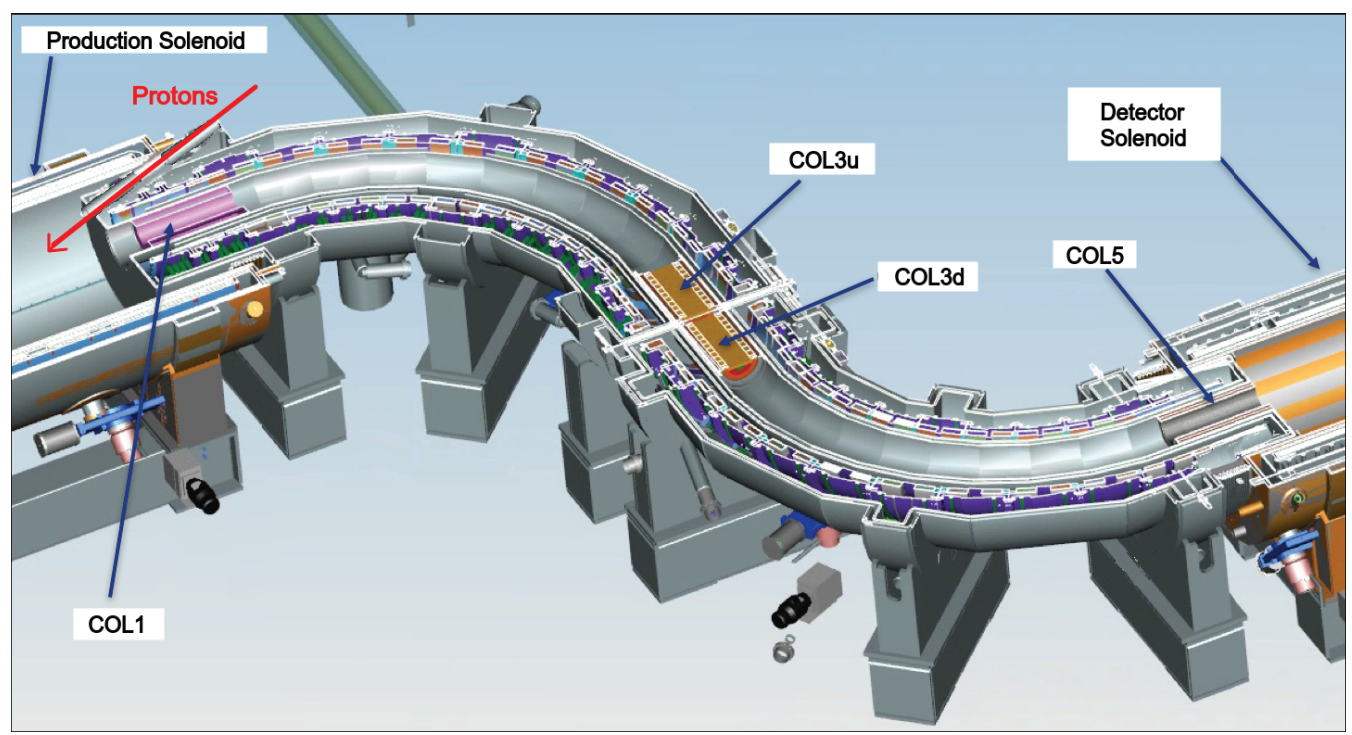

Figure 2.11: Representation of the Transport Solenoid. The collimators described in the text are here highlighted.

cm radius circular composition of copper wedges (Fig. 2.12, left). Besides filtering out high momentum particles, it also reduces the radiation damage for coils of the upstream part of TS (TSu). The unwanted energy deposition from these particles could ultimately lower the quench limit of the superconducting solenoid magnet.

- The first curved section (TS2) contains a toroidal field. The effect on particles crossing a $\pi / 2$ equivalent path is a vertical displacement $\mathrm{D}$ given by [42]:

$$
D=-\frac{\pi}{2} \frac{e}{Q} \frac{1}{0.3 B} \frac{P_{\|}^{2}+\frac{1}{2} P_{\perp}^{2}}{P_{\|}}
$$

where $\mathrm{B}$ is in Tesla and the components of momentum parallel and perpendicular to the magnetic axis, $P_{\|}$and $P_{\perp}$, are both in $\mathrm{GeV} / \mathrm{c}$.

Forward-moving particles $\left(P_{\perp} \sim 0 \mathrm{GeV} / \mathrm{c}\right)$ with higher momentum will suffer a stronger vertical displacement. According to 2.3 negatively charged particles are deflected upward. Non-charged particles, that cannot be bent by the magnetic field, will not be transmitted through TS2.

- The third part is another straight section (TS3) containing two identical and consecutive copper collimators (COL3u and COL3d) with a vertical offset of the apertures with respect to the horizontal center plane (Fig. 2.12). These are rotatable and can be used to select either positive or negative particles taking advantage of "curvature drift" acquired in the previous toroidal section. High momentum particles of both signs are also filtered. The collimators have an inner bore radius of $15 \mathrm{~cm}$ along the curved sides and an aperture of $20 \mathrm{~cm}$ between the upper and lower straight contours.

- The second toroidal section (TS4) will nearly undo the vertical dispersion, placing the muon beam axis in the center of the next section. 
- The last straight portion (TS5), with a lot of similarities with TS1, merges the last toroid with the Detector Solenoid and allow the muon flux to reach the Stopping Target. It contains a last polyethylene collimator (COL5) with an inner radius of $12.8 \mathrm{~cm}$, which will serve as an effective shield for the neutron backgrounds.
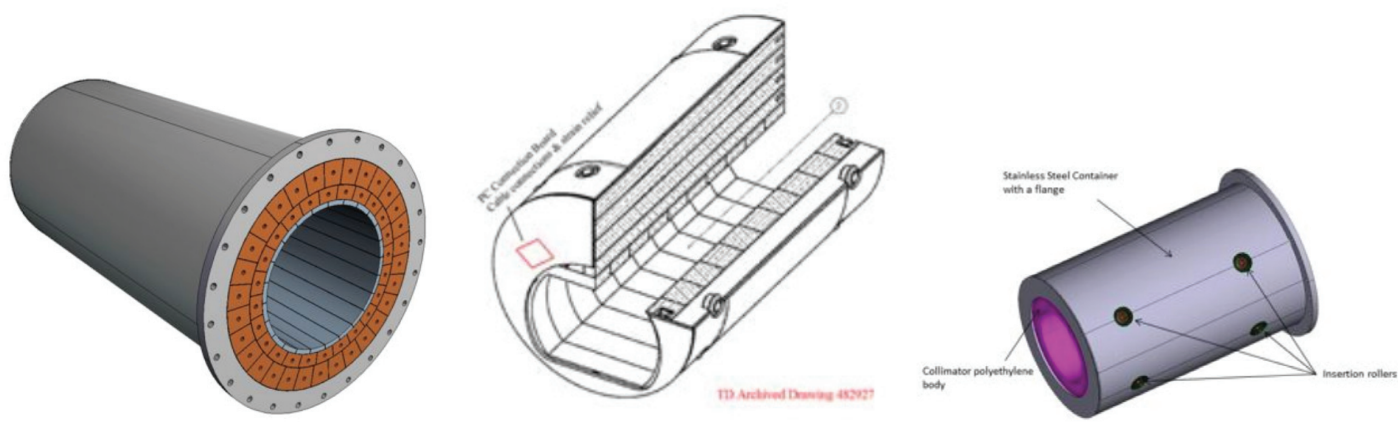

Figure 2.12: Design projects for the collimators inside the Transport Solenoid. Proceeding from left to right: COL1, COL3 (two consecutive identical pieces of it will be used, called COL3u and COL3d) and COL5.

TS also hosts a number of absorbers that are particularly important to suppress the antiproton background, that is one of the main subject of this thesis: because of their infinite lifetime and small kinetic energies the antiprotons can reach the Stopping Target at any time and produce background events evading the time selection cut. In addition, pions produced by the antiprotons interactions can also reach the stopping target so producing an out of time RPC background that cannot be suppressed by the time selection window.

A recent optimization maximizing the stopped muon yield and minimizing the antiproton induced backgrouns has led to the following absorbers configuration[43] :

- A first antiproton absorber foil is placed $1 \mathrm{~mm}$ upstream of COL1: it consists of a $250 \mu \mathrm{m}$ thick aluminum window, with a diameter of $30 \mathrm{~cm}$.

- An half-moon $20 \mathrm{~cm}$ long collar made of graphite is positioned inside the last bottom section of COL1: it has a inner radius of $120 \mathrm{~mm}$ and extends for 90 degrees. This collar suppresses antiprotons by a factor of 6 , while reduces the stopped muon rate by $\sim 4 \%$.

- The last absorber window is placed between COL3u and COL3d: it consists of a $150 \mu \mathrm{m}$ thick titanium window followed by 2 suspended aluminum foils of $127 \mu \mathrm{m}$. These foils provide a thickness increasing with the radial distance from the magnet axis, where the higher momentum particles are pushed according to Eq. 2.3.

\subsubsection{Detector Solenoid (DS)}

The Detector Solenoid is a $\sim 11 \mathrm{~m}$ long, $2 \mathrm{~m}$ radius cylindrical structure that holds the Stopping Target and the main Mu2e detectors. It consists of two sections: a "gradient section", located right after the TS and about $4 \mathrm{~m}$ long, and a "spectrometer section" of about $6 \mathrm{~m}$. The magnetic field at the entrance of the gradient section is $2 \mathrm{~T}$ and it decreases linearly to $1 \mathrm{~T}$; this allows the conversion electrons to be pitched forward into the acceptance of the tracker and, at the same time, rejects electron of higher momentum. 


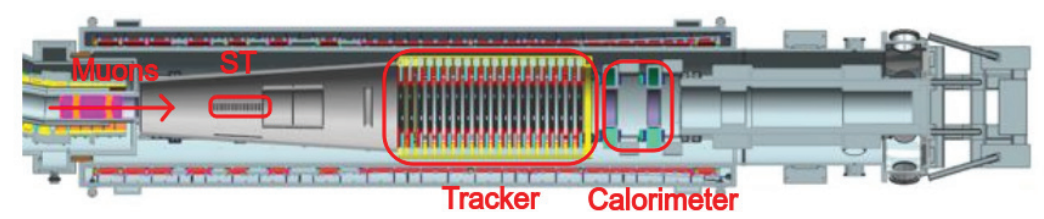

Figure 2.13: Representation of the Detector Solenoid internal structure. The muon beam comes from the left side of the picture and hits the Stopping Target. The tracker and the calorimeter disks are placed in the downstream "spectrometer section".

In the spectrometer section, the magnetic field is uniform over $5 \mathrm{~m}$ to allow a precise measurement of the particle momentum with the tracker.

It is worth noting that this solenoid has no bend, unlike COMET; therefore both $e^{+}$ and $e^{-}$can be observed simultaneously. The disadvantage is that the detector is exposed to the beam flash and to the remnant muon beam.

\subsubsection{Stopping Target (ST)}

The Mu2e Stopping Target (shown in Fig. 2.14), located in the half-way point of the "gradient section" of the Detector Solenoid, corresponds to the material where muons will be captured by the atoms and the conversion will eventually occur. It consists of 37 aluminum disks ( $75 \mathrm{~mm}$ radius and $100 \mu \mathrm{m}$ thick) with internal hole of radius 21 $\mathrm{mm}$ aiming to reduce the scattering of low momentum beam flash particles and then the radiation damage of the detectors.

The disks spacing will be $22.2 \mathrm{~mm}$ for a total target length of about $800 \mathrm{~mm}$.

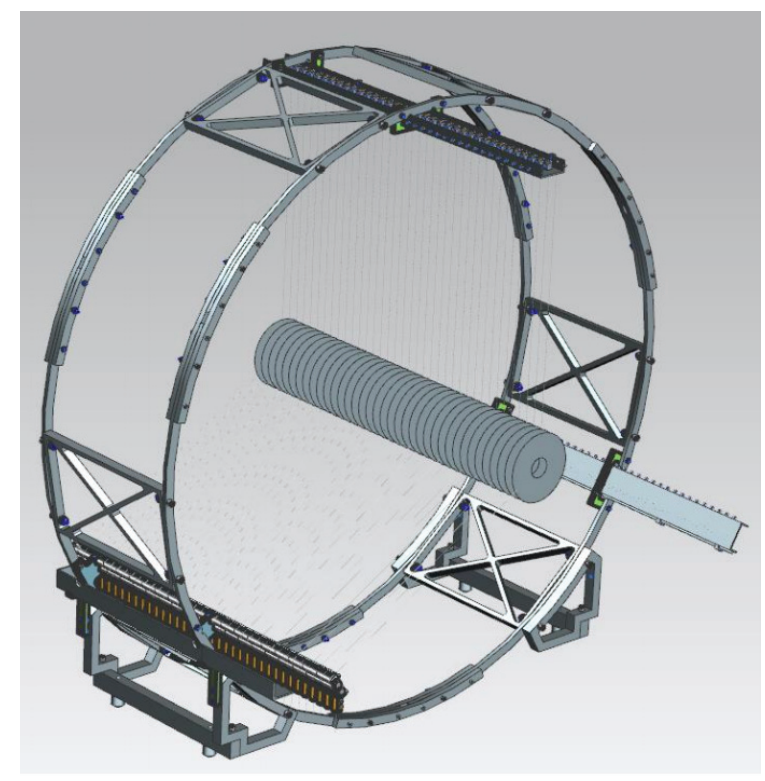

Figure 2.14: Design of the Mu2e Stopping Target and supporting wheel. Each one of the 37 aluminum foils is suspended with three cables (not visible in the figure).

The current design originates from two conflicting physics requirements:

- A maximized muon stopping probability, that asks for the maximum possible thickness. An indicative stopping fraction of $40 \%$ has been set in [44]). 
- A minimum amount of material crossed by the particles produced in the stopping target. This corresponds to minimize the energy loss inside the target and the effect of energy loss fluctuations on the momentum resolution.

The global muon beam line efficiency is estimated to be $1.594 \times 10^{-3}$ Stopped muons / $\mathrm{POT}^{5}$ (statistical uncertainty is of the order of $3 \%$ ).

\subsubsection{Inner and Outer Proton Absorbers}

The ST is followed by an empty cylindrical structure and surrounded by a conical polyethylene absorber (smaller at the upstream end and larger at the downstream end), respectively called Inner (IPA) and Outer Proton Absorber (OPA) (2.15), which are intended to reduce the flux of incoming and outgoing neutrons and protons. In particular, protons are produced in abundance (about 0.03 for each muon captured) and can generate spare hits in the tracker or in the cosmic ray veto.

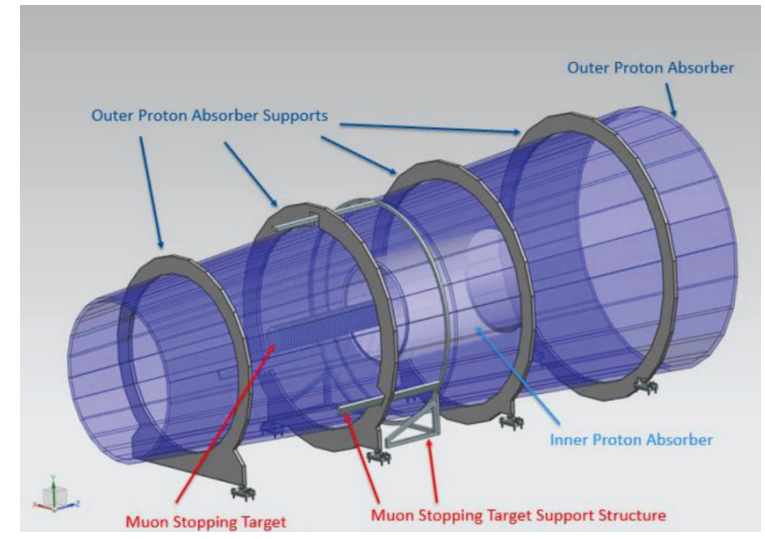

Figure 2.15: Structure of the Inner and the Outer Proton Absorber.

\subsection{The Mu2e Tracker}

\subsubsection{Requirements}

The Straw Tube Tracker is the detector devoted to the measurement of the particle momenta. Physical requirements on this detector come from the ability to separate the CE monochromatic signal from the DIO electrons. Since DIO spectrum falls as $\left(E_{\text {conv }}-E\right)^{5}$ near the endpoint, the corresponding background increases very quickly as the resolution degrades. In order to reach the required DIO suppression, the experiment will need a resolution well below $1 \mathrm{MeV} / \mathrm{c}$ [37], the more dangerous part being its right-side tail, that can push the abundant low momentum DIO electrons into the signal region.

Also the left-side tail, mainly due to the energy loss in the Stopping Target and in the tracker material must be kept under control since it smears the monochromatic CE energy downwards and, even if the DIO spectrum is smeared down as well, the stochastic nature of this process increases the overlap of the two distributions. This translates into the requirement to keep the energy loss in the tracker as small as possible, justifying the choice of a straw tube tracker.

\footnotetext{
${ }^{5}$ This number is the output of a MC simulation of $10^{8}$ POT.
} 


\subsubsection{The straw tubes structure}

The fundamental element is a $25 \mu \mathrm{m}$ gold plated tungsten wire (anode) centered in a $5 \mathrm{~mm}$ diameter tube called "straw" (cathode). The straws are filled with a drift gas of 4:1 Argon: $\mathrm{CO}_{2}$ mixture and delimited by two layers of $6 \mu \mathrm{m}$ Mylar separated by $3 \mu \mathrm{m}$ of adhesive. The tracker structure is shown in Fig. 2.16: groups of 96 straws with length varying from $334 \mathrm{~mm}$ to $1174 \mathrm{~mm}$ are placed together, with $1 \mathrm{~mm}$ gap in between, to form a $120^{\circ}$ arc called "panel"; the complete plane is obtained combining three panels rotated by $120^{\circ}$; a tracker "station" is composed of two planes rotated by $60^{\circ}$. Finally 18 consecutive stations form the $3.27 \mathrm{~m}$ tracker structure.
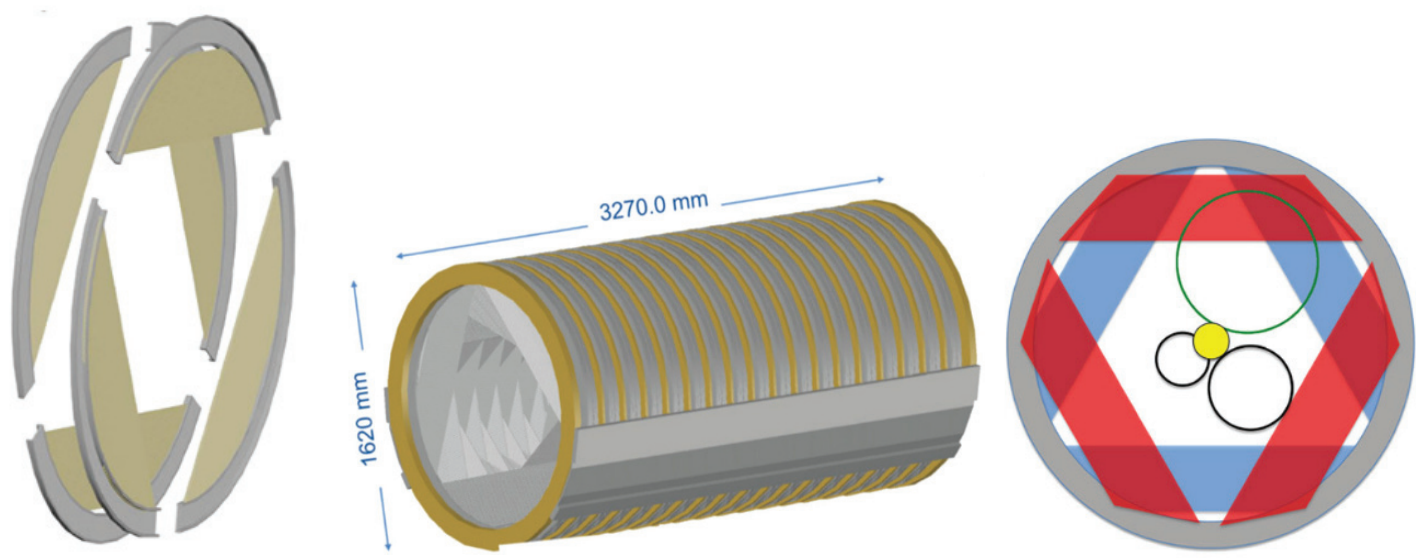

Figure 2.16: The Mu2e tracker. (LEFT) Two panels assembled in a station. (CENTER) The assembled tracker. (RIGHT) Transversal view of a station: the three circles are projections of tracks at the Michel peak (small black circle), an intermediate momentum, and the conversion energy (last in green).

The high beam intensity required to collect the huge number of muon stops needed to improve the limit on muon to electron conversion will also expose the detector area to huge flux of particles, directly coming with the beam (beam flash) or produced by the capture of the muons in the $\mathrm{Al}$ nuclei.

The annular design allows the passage of the low momentum charged particles without striking the detector elements. For example, the vast majority of the electrons coming from muon decays in orbit (Fig. 1.10) has a momentum too low to enter in the tracker detector. (right side of Fig. 2.16).

\subsubsection{Readout system}

The tracker readout system provides information about the pulse timing at the end of each straw and total height. Each pulse timing is stored and both difference and sum contain useful information: the difference is computed in order to measure the longitudinal position along the wire, while the sum depends on the particle arrival time and the drift distance. The pulse height is also measured, to provide $d E / d x$ for particle identification.

Fig. 2.17 schematically shows the tracker front-end electronics here briefly discussed: signals arriving to the extremities of each straw tube are individually amplified and sent to the respective TDC. In parallel, the analogical sum of the signals is sent to an ADC. All the digitized information are sent to a digitizer board that transmits the digitized waveform to the data acquisition system. 


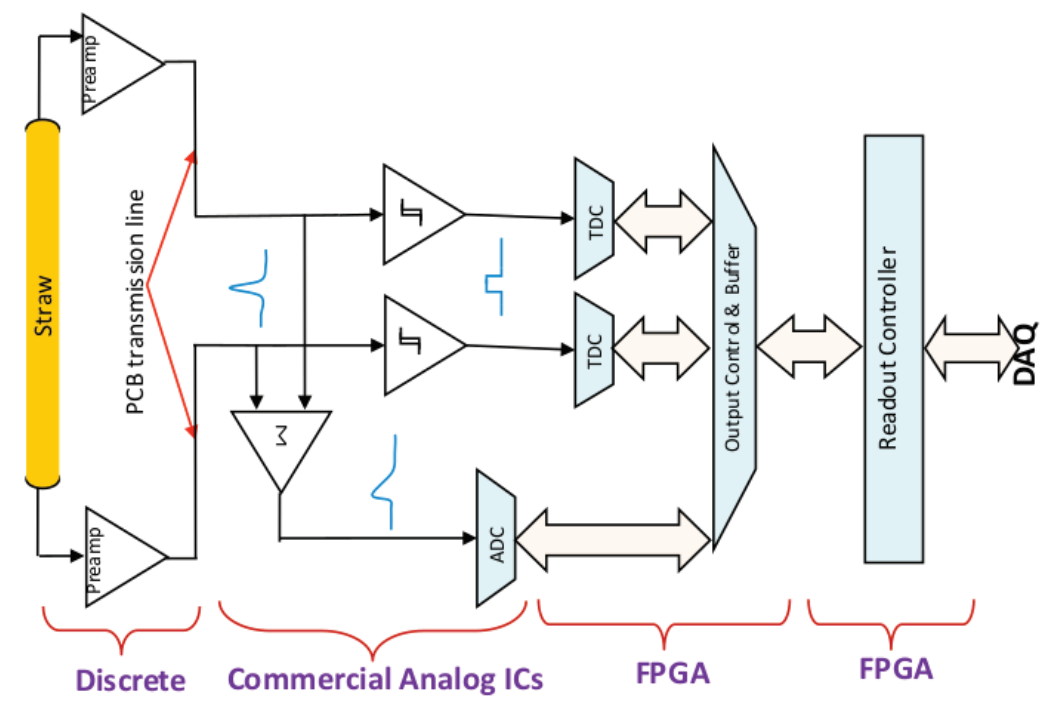

Figure 2.17: Signal flow through the tracker front end electronics.

\subsubsection{Resolutions}

The left side of Fig. 2.18 shows the difference between the particle true (MC) and reconstructed (obtained by the fit) momentum at the entrance of the tracker: the distribution has been obtained using $10^{6}$ simulated conversion electrons events ${ }^{6}$ : the core width $(159 \mathrm{keV} / \mathrm{c})$ and the tails relative importance allow to suppress the DIO background at the required level.
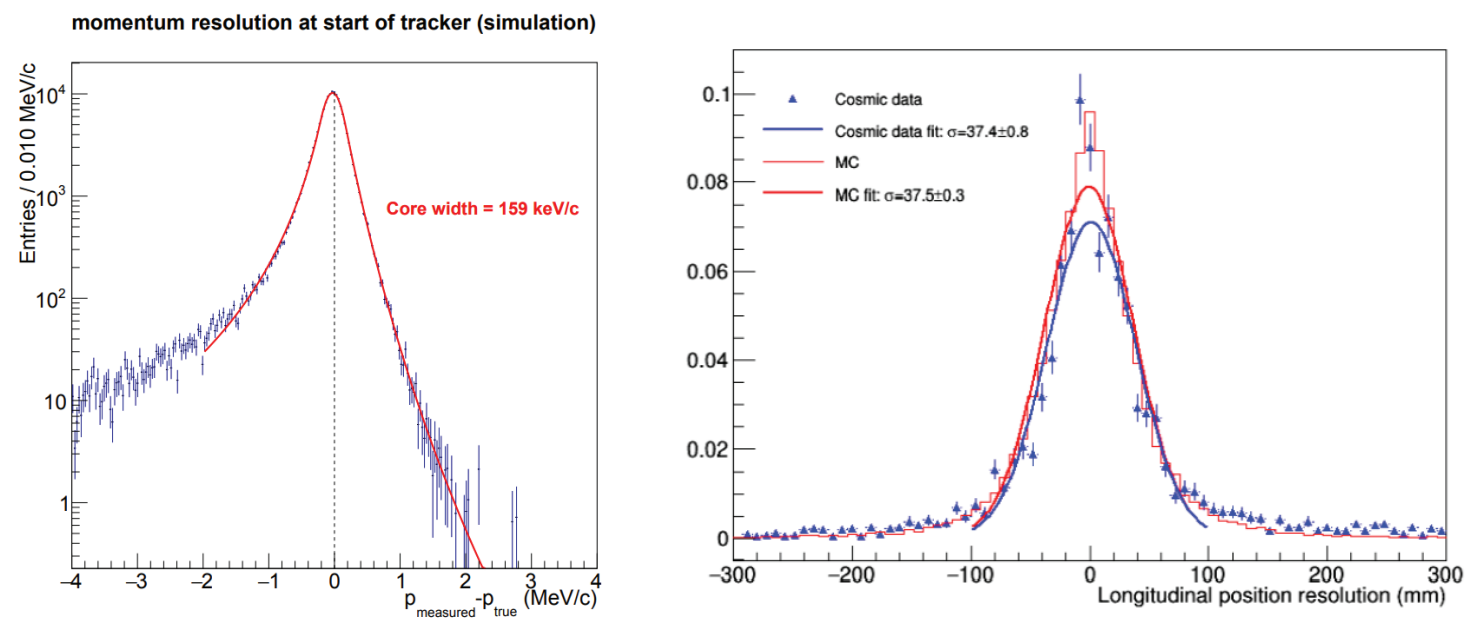

Figure 2.18: [LEFT] Last "blessed" expected resolution of the Mu2e tracker for electrons at the conversion energy (from a simulation with $10^{6}$ events, in 2018). Both background overlay and selection cuts are included. [RIGHT] The longitudinal resolution obtained from the 8-Channels module.

In 2016 a small tracker prototype with only eight straws ('8-Channels' module) was built in the Berkeley Laboratory and used to test the final readout system and to measure

\footnotetext{
${ }^{6}$ The noise hits coming from overlapping background events have been included; track quality selection cuts described in the next chapter have been applied.
} 
the hit position resolution for a single straw tube. Right side of Fig. 2.18 shows the longitudinal resolution of the tracker as obtained from data: the extracted width of $\sigma_{\text {long }}=37 \mathrm{~mm}$ is in good agreement with Monte Carlo expectations. The major effects affecting the longitudinal resolution are the rise time of the pre-amplifier and the noise fluctuation.

\subsection{Mu2e detectors: Calorimeter}

\subsubsection{Tasks and requirements}

The Mu2e calorimeter has been designed to help the tracker to suppress the $\mathrm{CE}$ background and has various tasks that can be summarized as follows[37]:

1. Reconstruct the particle impact point with a resolution better than $1 \mathrm{~cm}$ to unequivocally associate the tracker track to the energy deposited by the particle in the calorimeter;

2. Reconstruct the conversion electron arrival time with a resolution better than $0.5 \mathrm{~ns}$ and its energy deposit with a resolution of $\sigma_{E} / E \sim 10 \%$ to provide, when combined with tracker information on time and momentum, a rejection factor better than 200 on cosmic muons surviving the Cosmic ray veto;

3. Provide a fast trigger to the experiment.

\subsubsection{Calorimeter structure}

The Mu2e calorimeter consists of two disks separated by half the wavelength for a 105 $\mathrm{MeV} / \mathrm{c} \mathrm{CE}$ in a $1 \mathrm{~T}$ field $(\simeq 70 \mathrm{~cm})$. The disks inner radius is $35 \mathrm{~cm}$, the outer $66 \mathrm{~cm}$. This structure maximizes the acceptance for CEs in such a way that if a conversion electron passes through the hole of the first disk it will detected by the second. A schematic is shown in Fig. 2.19.

Each calorimeter disk is composed of 674 pure CsI square crystals $(3.4 \mathrm{~cm} \mathrm{x} 3.4 \mathrm{~cm}$ and $20 \mathrm{~cm}$ long) wrapped with a $150 \mu \mathrm{m}$ foil of Tyvek. ${ }^{7}$ Since the CsI radiation length is $X_{0} \sim 2 \mathrm{~cm}$ and considering that the average incident angle for the conversion electrons is $50^{\circ}$, the effective material length seen by the electron shower is $\sim 300 \mathrm{~cm}$ corresponding to $15 X_{0}$, which is considered acceptable.

A $2 \mathrm{~mm}$ air gap is maintained between the crystal and the readout sensor to reduce thermal coupling between crystals and electronics and to avoid using any optical glue that could deteriorate the vacuum because of the outgassing or the light transmission because of its opacity increasing with radiation damage.

\subsubsection{Readout system}

A single crystal is red by two custom UV-extended silicon photo-multipliers (SiPM), each composed of a $2 \times 3$ array of individual $6 \mathrm{~mm} \times 6 \mathrm{~mm}$ cells. Each cell consists of a planar matrix of 14400 avalanche photo-diodes (APD) pixels $(50 \mu \mathrm{m} \times 50 \mu \mathrm{m})$, operating in Geiger mode. Pixels are connected in parallel so that the resulting signal equals the sum

\footnotetext{
${ }^{7}$ Pure CsI, with its wavelengths peak emission at $315 \mathrm{~nm}$ and scintillation time of about $20 \mathrm{~ns}$, has been preferred to other inorganic crystals because of its fast emission and relatively cheap cost.
} 


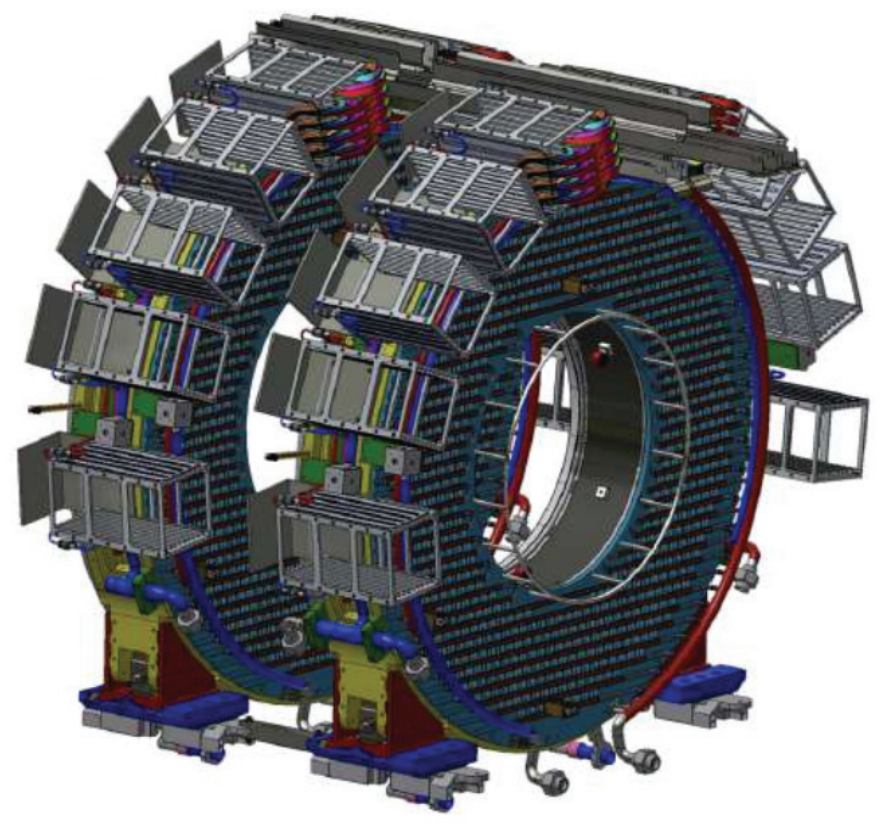

Figure 2.19: Front view of the two disks of the Mu2e electromagnetic calorimeter.

of all contributions. Solid state photo-detectors have been preferred to photomultiplier tubes (PMT) because of the operation conditions inside a $1 \mathrm{~T}$ magnetic field. To connect different cells two competing requirements on capacitance on operating voltage must be considered: a parallel connection would keep the voltage low but increase the capacitance and then the sensor response time; a series connection would decrease the capacitance but increase the required voltage. The adopted solution is a compromise: a parallel arrangement of two groups of three cells biased in series.

Each SiPMs is connected to an independent power supply and high voltage amplifier located in Front-End Electronics (FEE) board directly connected to it. In order to reduce the dark current and the effects of neutron radiation the SiPMs will be operated at a temperature lower than $0{ }^{\circ} \mathrm{C}$.

A crystal readout unit with 2 SiPMs, 2 FEE boards, the mechanical support that also ensures the SiPM cooling, the Faraday cage used to reduce the noise and a fiber used for the laser calibration is shown on the left of Fig. 2.20.

Groups of 20 amplifiers are controlled by a dedicated Mezzanine Board (MB), from where signals are sent to the ADCs located in the digitizer board (DIRAC). Both boards are arranged in the electronic crates surrounding the calorimeter disks (Fig. 2.19).

The DIRAC boards are also responsible for the zero suppression and the data transmission, via optical fibers, to the main DAQ of the experiment. An example of digitized waveform coming out from the digitizer is shown on the right of Fig. 2.20.

\subsubsection{Resolutions}

Both the crystal hit energy and time are measured by fitting the waveform with an average waveform template obtained from the data themselves. The best time resolution is obtained using a fit range limited to the waveform rising edge. A slightly larger fit interval gives the best results for energy reconstruction. 

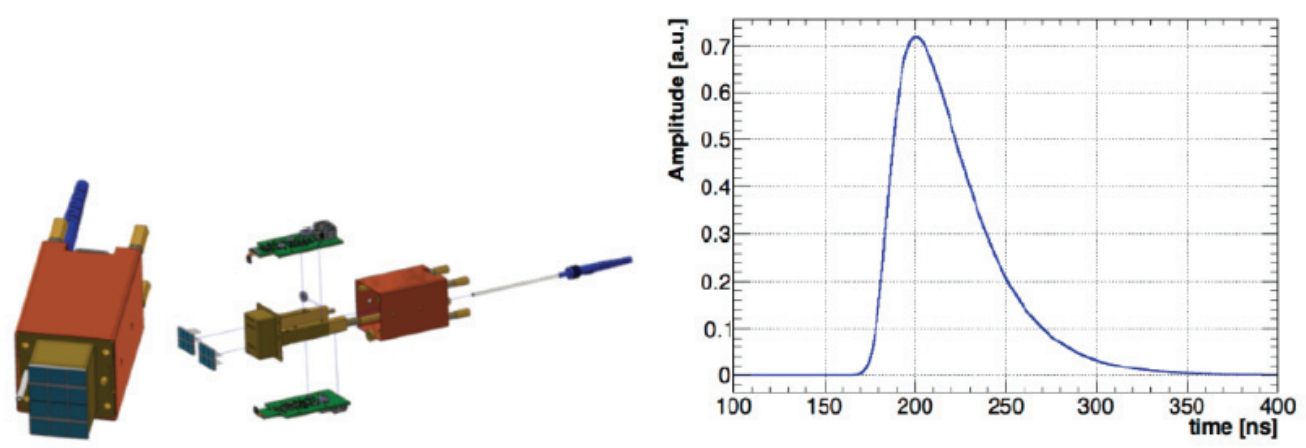

Figure 2.20: (LEFT) Assembled and exploded view of the FEE holder showing the two SiPMs glued on the inner cupper support, the pair of FEE boards, a Faraday cage and a needle for insertion of the optical fiber. (RIGHT) A simulation of the output waveform from a CsI crystal $+\mathrm{SiPM}+$ electronics chain including the digitizer.

The measuremnt of the two sensors reading the same crystal are averaged to obtain the crystal hits.

Crystals hits are grouped in time and position to get the shower energy and time.

Fig. 2.21 shows the difference between the CE reconstructed energy and the CE energy obtained by Monte Carlo, including also the overlapping energy deposits coming from other beam particles.

An energy resolution of $\sim 5 \mathrm{MeV}$ (RMS) and a time resolution of $\sim 110$ ps have been obtained.

The corresponding spatial resolution, obtained by the energy weighted mean position of the crystals belonging to the same clsuter, is $\sim 6 \mathrm{~mm}$.

All values are well below the requests.
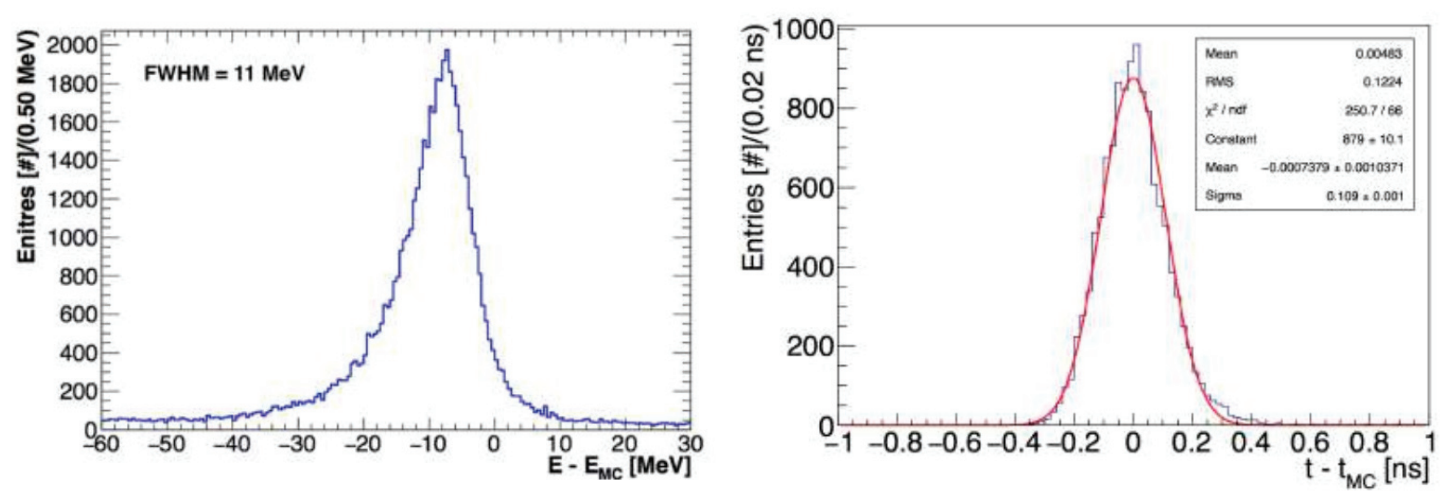

Figure 2.21: Distribution in energy (LEFT) and time (RIGHT) residuals between the reconstructed variable and the Monte Carlo value.

\subsubsection{Quality controls}

The Quality Control (QC) procedure for the CsI crystals is taking place in the Mu2e Calorimeter laboratory at Fermilab. The set of specifications, described in [45], are checked during the qualification tests: 
- Mechanical dimensions and shapes are particularly important for the alignment within the disk and to avoid extreme stresses of the crystal surfaces. Deviation from a perfect 3-dimensional parallelepiped is required to be less than $100 \mu \mathrm{m}$. To secure this, each crystal face was inspected with a Coordinate Measuring Machine.

- Scintillation properties are crucial to reach the required energy and time resolution. In this context, studies have been focused on: Light Yield, Longitudinal Response Uniformity (affected by impurities), energy resolution and relevance of the CsI slow component [46].

- Dedicated tests have revealed that the ionization produced by the beam particles (Radiation Induced Noise, RIN) is the major noise component. This is tested using a ${ }^{60} \mathrm{Co}$ source.

- The deterioration of the light response with the total ionizing dose (Radiation hardness) has then been performed on a small randomly selected sample for each production lot.

Also the UV extended SiPMs have undergone a mass quality test. A dedicated automatic test station (Fig. 2.22), built in Pisa, is able to simultaneously host $25 \mathrm{SiPMs}$ and check them at three different temperatures $\left(25^{\circ} \mathrm{C}, 0{ }^{\circ} \mathrm{C}\right.$ and $\left.-10{ }^{\circ} \mathrm{C}\right)$. Each $\mathrm{SiPM}$ cell is individually tested thanks to a relays system (driven by a micro controller) that allows to switch on and off the contacts of the different pins. The light source used to illuminate the SiPM matrix is a green LED. The matrix illumination is made uniform using two planes of sanded quartz. The central SiPM and the ones at the four board corners have been manually calibrated and used as a reference for the stability and the uniformity of light; deviations are expected to be smaller than $5 \%$. At the moment of writing all sensors have been tested and only 48 out of 3950 have been rejected.

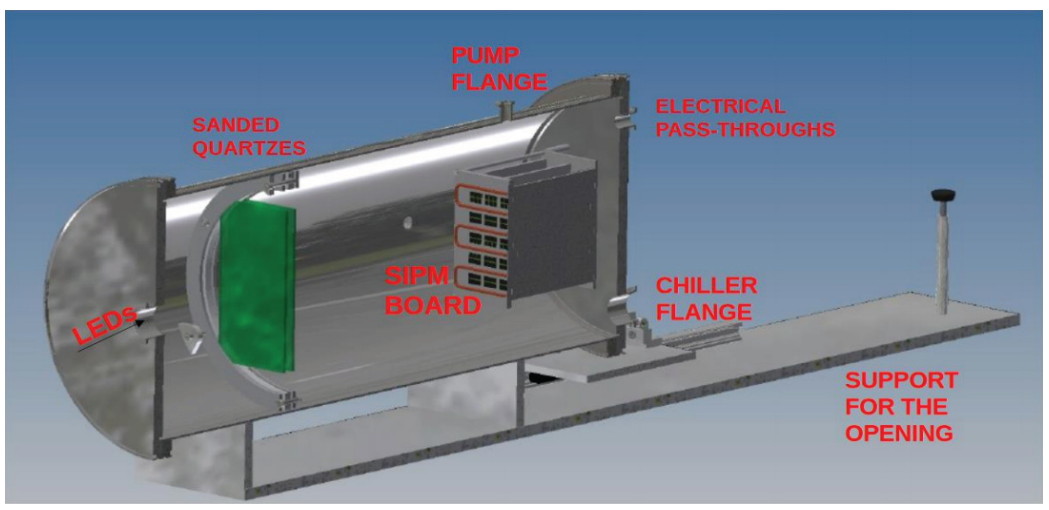

Figure 2.22: QA station designed for the tests of the calorimeter SiPMs as described in the text.

The quality measurement have included:

- The I-V characteristics, performed for each Mu2e SiPM cell and used to determine the breakdown voltage $\left(V_{b r}\right)$, i.e. the point where the curve behavior changes from a convex function to a concave one. The operational voltage is then defined as $V_{o p}=$ $V_{b r}+3 \mathrm{~V}$.

- A measurement of the Gain $\left(>10^{6}\right) \times$ the Photon Detection Efficiency (PDE, $>20 \%$ at $315 \mathrm{~nm}$ ) has been performed by looking at the ratio of the currents pulled by 
a cell and the closest reference sensor, while illuminating both with a stable and uniform light.

- A Mean Time To Failure (MTTF) test is necessary to assure the sensors can run for at least one year without need for a repair: 15 pieces, randomly selected from each production lot, have been kept in a dedicated station for $\sim 18$ days at a temperature of $65{ }^{\circ} \mathrm{C}$. The resulting MTTF corresponds to $10^{6}$ hours at the experiment temperature $\left(0{ }^{\circ} \mathrm{C}\right)$.

A small size calorimeter prototype, named Module-0, was built at the National Laboratories of Frascati (Italy) in May 2017, using 51 crystals and 102 photosensors from different vendors. The module has been exposed to an electron beam with energies between $60 \mathrm{MeV}$ and $120 \mathrm{MeV}$, at two different angles: $0^{\circ}$ (for calibration) and $50^{\circ}$ (as in the experiment). Detailed MC comparisons and data analyses are deeply discussed in [46] and here shown in Fig. 2.23.
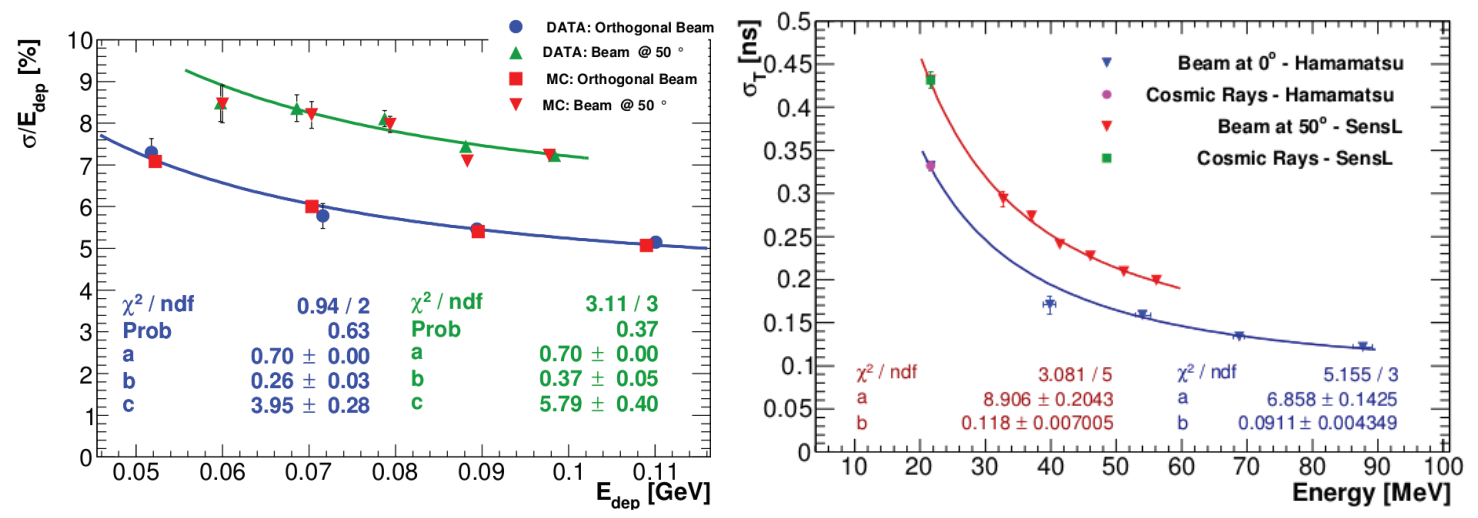

Figure 2.23: (LEFT) Energy resolution as a function of the mean energy deposit in the Module-0 in the orthogonal (blue) and tilted (green) configuration and comparison with the MC expectation.

The energy resolution dependence on the mean energy deposition was parameterized with:

$$
\frac{\sigma_{E}}{E}=\frac{a}{\sqrt{E}[G e V]} \oplus \frac{b}{E[G e V]} \oplus c
$$

where the coefficient "a" represents the stochastic term, "b" the noise term and "c" the constant term ${ }^{8}$.

The time resolution trend with the highest energetic deposit was fitted with:

$$
\sigma_{T}=\frac{a}{E[G e V]} \oplus b
$$

where "a" is proportional to the emission time constant of the un-doped CsI and "b" takes the additional readout electronics contribution into account. All these results are in good agreement with the Mu2e requirements.

The Module 0 is currently being refurbished with the final qualified crystals and SiPMs and the final front end and digital electronics and will be exposed to a new test beam, hopefully in the next year.

\footnotetext{
${ }^{8}$ In reality, the fit was insensitive to thre stochastic term and "a" was fixed to $0.7 \%$ based on the light yield contribution of 20 p.e./ MeV
} 


\subsection{Reconstruction}

The track reconstruction is divided into two steps: hits matching in time and position are first grouped in track candidates by the pattern recognition algorithm and then track parameters are extracted by a fit that also takes into account the energy loss in the tracker material.

Both procedures makes use of the Kalman method: a special procedure that improves the knowledge about the trajectory (or track parameters) proceeding progressively from one measurement to the next. In practice, the $\mathrm{n}_{t h}$ hit position is predicted by the pattern recognition algorithm extrapolating the helix trajectory from the previous n-1, the closest hit in $100 \mathrm{~mm}$ is added to the track and the helix information are updated. The Kalman fit adopts the same logic: first, an estimate of the impact of the $\mathrm{n}_{t h}$ measurement on the track parameters is made based on knowledge of the current state and then they are re-calculated based on a weighted combination of the measurement and predicted values, the figure of merit being a " $\chi^{2}$ type" variable.

The pattern recognition can be done either with a standalone tracker algorithm or with the tracker being driven by the calorimeter cluster information:

- In the Tracker-only pattern recognition algorithm a hits triplet is initially made of the stopping target center, the hit closest to the tracker entrance and a third variable point. Starting from these, the pattern recognition progressively extrapolates the helix and adds new points until 15 hits are collected.

- Calorimeter driven (CalPatRec). With the time information of the calorimeter it is possible to constrain accepted hits to be compatible with an independent measurement. If a calorimeter cluster with $\mathrm{E}>50 \mathrm{MeV}$ is identified, this is provided as a starting seed for the pattern recognition procedure. The only accepted hits are the ones that satisfy the two following conditions ( $\Delta t$ is the time difference between the straw hit and the calorimeter cluster and $\Delta \phi$ is the difference in azimuthal angle):

$$
|\Delta t|<40 \mathrm{~ns} \quad|\Delta \phi|<\frac{\pi}{2}
$$

This reduce background hits by a factor $\sim 20$, without significantly affect CE efficiency [47]. The impact of such a procedure is shown in Fig. 2.24.

The calorimeter can also assist the tracker in the reconstruction by providing an additional hit to be used in the track fit. The procedure to identify this extra point, called TrkCaloHit, is divided into the following phases:

- In the transversal plane of the calorimeter, the $(\mathrm{X}, \mathrm{Y})$ position of the "cluster hit" is identified with the energy weighted average among crystals. This identify an unique transversal axis to the calorimeter.

- The point on this axis that minimize the distance from the extrapolated track trajectory is defined as the Point Of Closest Approach (POCA).

- The distance between POCA and the crystal front face finally fixes the last coordinate $\mathrm{Z}$.

- The time information is taken from the cluster time. 

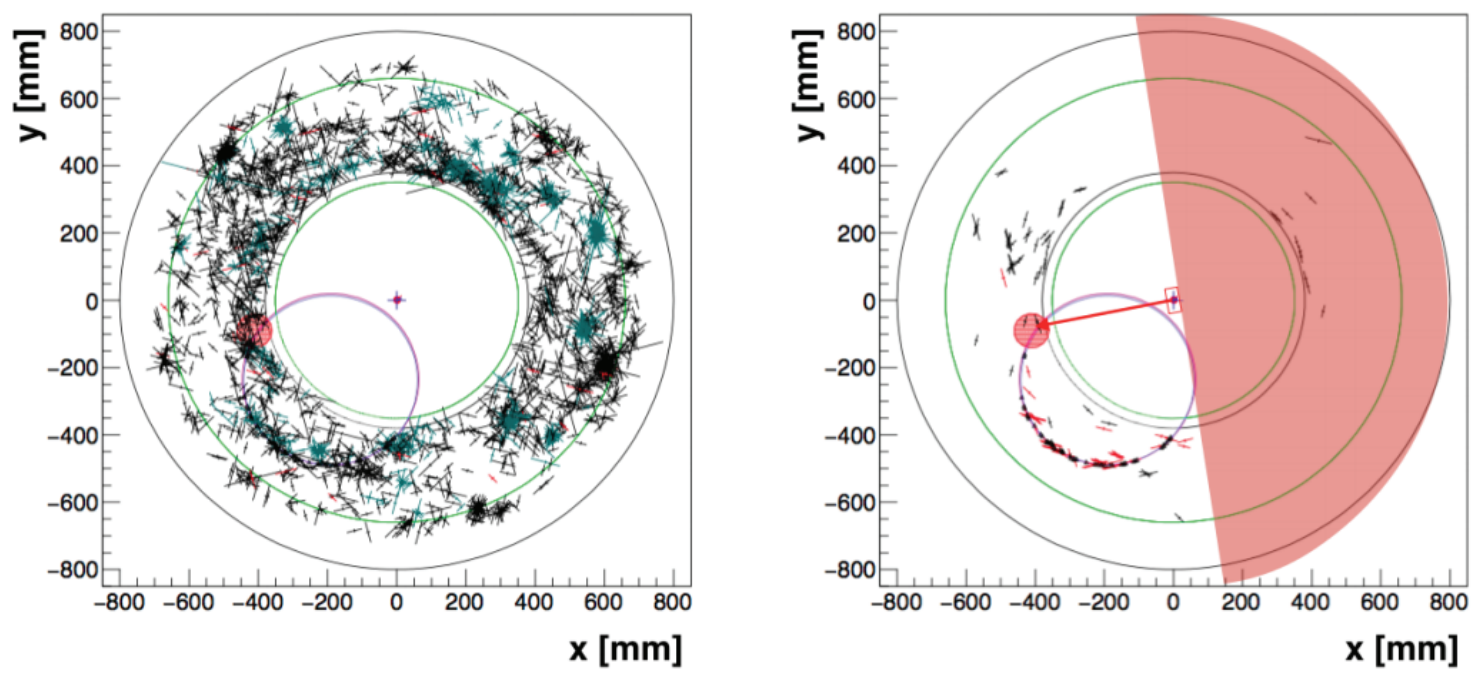

Figure 2.24: A transverse view of the event display for a CE + background, without (left) and with (right) using the CalPatRec algorithm. Black crosses are the straw hits, the red bullet is the calorimeter cluster and the red semicircle is the section removed by the $\Delta \phi$ selection.

The strength of the method originates from the fact that the calorimeter time resolution is better than the one of the tracker and that events with better resolution ultimately count more in the Kalman fit (which assign higher weights to lower time resolution hits). Then, adding the so defined "cluster hit" to the fit procedure, introduces an highly weighted event that reduces the overall time resolution of the tracker (Fig. 2.25) from $\sigma_{t_{0}} \sim 0.9 \mathrm{~ns}$ to $\sigma_{t_{0}} \sim 0.5 \mathrm{~ns}$.
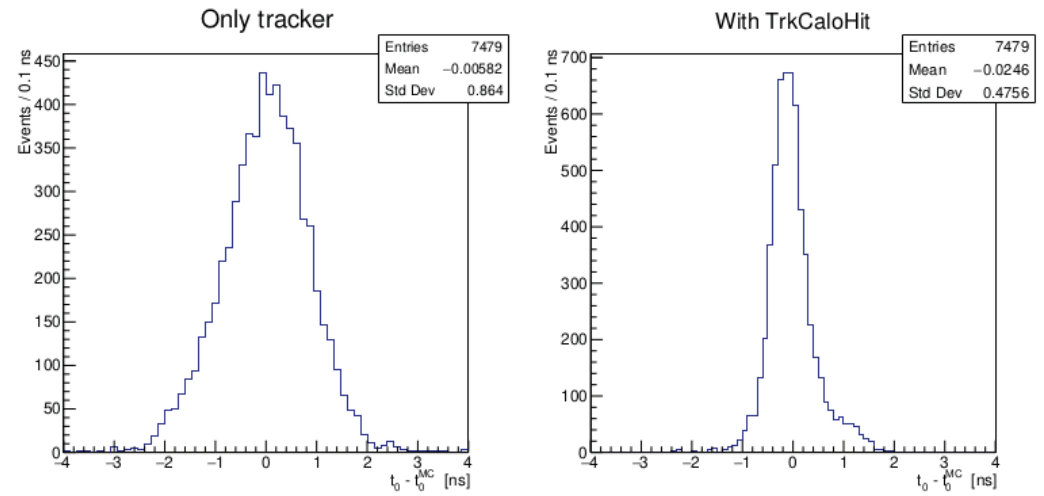

Figure 2.25: Time residuals (time at the center of the tracker for the reconstructed tracks and MC truth information) if only the tracker reconstruction algorithm is used (LEFT) or if the TrkCalHit method is introduced (RIGHT).

\subsection{Mu2e detectors: Stopping Target Monitor}

As shown in Section 1.2.3, Mu2e will normalize its measurement of CE events with the number of nuclear muonic captures in the aluminum target during the measurement period. Since the capture rate for $\mathrm{Al}$ is known from literature ( $\sim 61 \%$ of stops), it is sufficient to measure either the number of stops or the number of captures. 
To measure the number of stopped muons one can look for the $347 \mathrm{keV}$ X-rays produced from the $2 \mathrm{p} \rightarrow 1$ s transition of the muon being captured by the aluminum atom. This happens for the $80 \%$ of the stopped muons.

Nuclear captures can be measured through two different processes:

- a $1.81 \mathrm{MeV}$ gamma-rays is emitted in the following nuclear capture chain:

$$
\mu^{-}+{ }^{27} \mathrm{Al} \rightarrow{ }^{26} \mathrm{Mg}^{*}+\nu_{\mu}+n \quad{ }^{26} \mathrm{Mg}^{*} \rightarrow \mathrm{Mg}+\gamma(1809) .
$$

The de-excitation time of ${ }^{26} \mathrm{Mg}^{*}$ is negligible (476 fs) so this process has an effective mean-life equal to that of the muonic atom (864 ns). It occurs for the $51 \%$ of the nuclear captures, i.e for the $31.1 \%$ of the stopped muons.

- a $844 \mathrm{keV}$ gamma-ray comes from the decay of long lived (9.5 minute half-life) isotopes produced in muon nuclear captures:

$$
{ }^{27} \mathrm{Mg} \rightarrow{ }^{27} \mathrm{Al}+\gamma(844)+e^{-}+\bar{\nu}_{e}
$$

This involves the $9.2 \%$ of nuclear captures or the $5.7 \%$ of stopped muons.

Fig. 2.26 offers an handle on all these different processes.

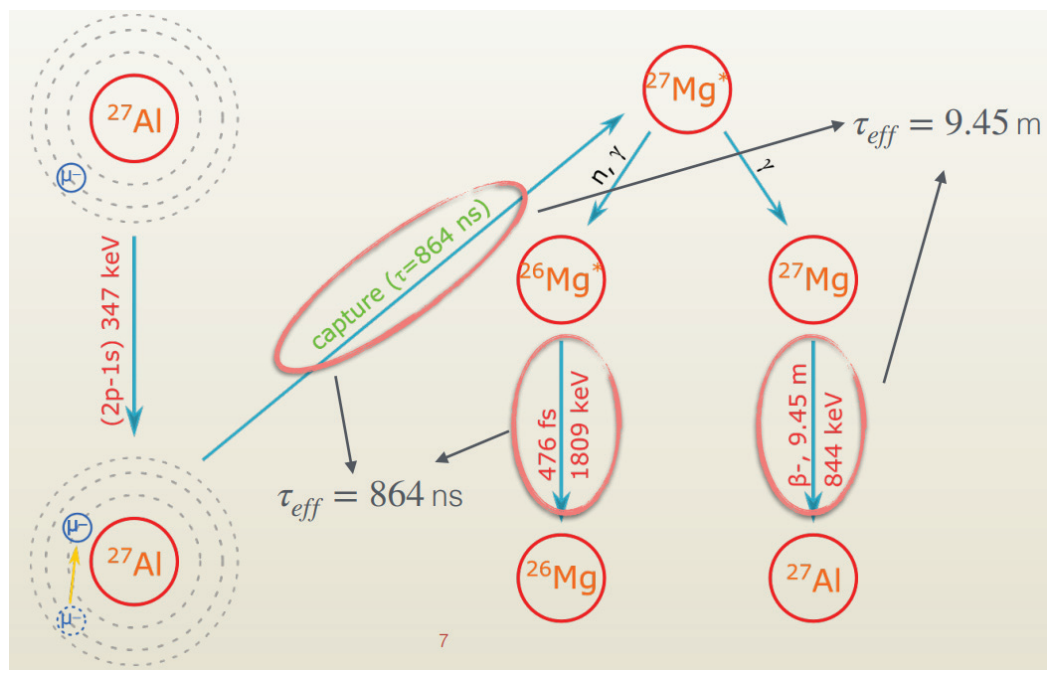

Figure 2.26: Scheme of the three X-ray lines described in the text. The figure also follows the history of a the Aluminum atom after the muon is bound in orbit. The figure does not cover all the existing processes.

It is important to remind that the total number of stopped muons, in a given proton pulse interval, consists of 'old' muons left over from previous proton pulses, plus 'new' muons produced by the current pulse. This justifies the choice of measuring different components with different time constants. As described in [48], it is also interesting to exploit the "off-spill" time period with no beam, i.e. the 12 tick dedicated to No $\nu$ a as described in Sec. 2.2, to perform a measurement of the delayed photons with a better S/B ratio.

The Stopping Target Monitor (STM), located 34 m downstream the DS after appropriate collimators, is the detector designed to make this measure with an accuracy better than $10 \%$ [49]. Two High Purity Germanium detectors (HPGe, FWHM $\simeq 2.5 \mathrm{keV}$ for energies 
between $300 \mathrm{keV}$ and $2 \mathrm{MeV}$ ) aim for measures of the $347 \mathrm{keV}$ and $844 \mathrm{keV}$ lines, which require a good energy resolution due to the presence of nearby contamination. A LaBr detector has a $\sim 10 \mathrm{x}$ poorer resolution compared to HPGe but a much higher rate capability, for this reason it is well suitable for measuring the prompt $1.81 \mathrm{MeV}$ line: its lower energy resolution is compensated by the higher photon energy.

\subsection{Mu2e detectors: Cosmic Ray Veto}

During the Mu2e running time, cosmic rays will rain down upon the detector area at a rate of $\sim 15000 /$ s producing roughly one fake CE per day. Processes of interest may be a muon decay, a muon mis-identification as a CE or a $105 \mathrm{MeV}$ electron knocked out of the DS material. Most important, cosmic rays are not related to the muon beam and the associated background scales only with the experiment live time (and not with the beam intensity).

The Cosmic Ray Veto (CRV) is formed by 83 modules, covering the DS area and the last part of the TS. Each module is composed of 4 layers of polystyrene scintillator counters coated with $\mathrm{TiO}_{2}$, embedded with wavelength shifting fibers and red out by SiPMs. Aluminum absorbers are placed between the scintillator layers. Fig. 2.27 offer a representation of the CRV modules (on the left) and their inner structure (on the right). To veto an event, at least three layers are required to be active: each layer has an efficiency
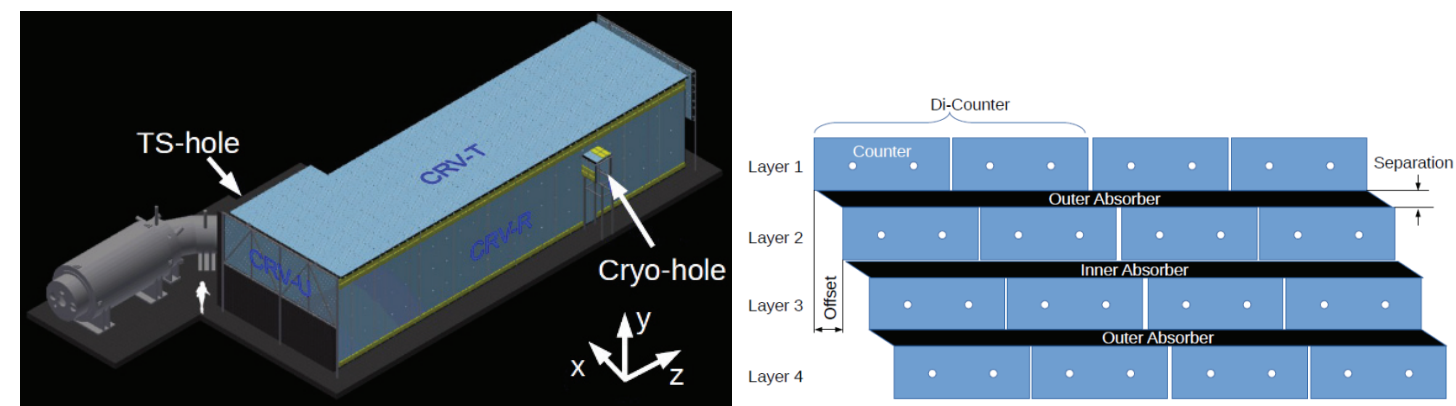

Figure 2.27: (LEFT) 3D view of the different modules of the Cosmic Ray Veto. (RIGHT) Scheme of the layers structure of the CRV. A $42 \mathrm{~mm}$ shift is present between different layers.

of $99.5 \%$, so the CRV total efficiency is $99.99 \%$ [50].

Since cosmic rays represent the most important background for Mu2e, the CRV has very demanding requirements on its veto efficiency or, equivalently, on the light yield of its counters. The CRV efficiency and light yield can be preserved by lowering the hit threshold, but this is possible only until the point where either the SiPM noise rate becomes unacceptable for the front-end electronics, or the false veto rate becomes too high, causing an unacceptably large dead time for the whole experiment.

Another possible source of dead time comes from the hits produced by the neutrons coming from the interactions in the production target: this neutron induced noise can be mitigated using a concrete shielding between the PS and the CRV.

CRV performances have become even more critical after the decision to run Mu2e in two phases, with an initially reduced beam intensity resulting in a longer total run period.

CRV light yield deterioration with time (aging) has been measured to be significantly faster than anticipated: the current understanding is that internal stresses within the 
scintillator, left by the extrusion process, produce crazings which results in internal reflections, reducing the amount of light reaching the fiber.

Using the results of the last masurements [51], an aging of 8.7\%/year (compared to the previous value of $3 \%$ ) is assumed in order to compute the year-by-year PE yield.

The dead time for the first run phase will be around 4\%, well within the $10 \%$ requirement.

By the end of the high-intensity run, the photo-electron light yield it is estimated to be only $35 \%$ of its original value, with an unacceptable increase of the dead time. Different aging mitigation strategies are currently under study. 


\section{Chapter 3}

\section{Signal and background analyses}

In this chapter we will study the characteristics of the conversion electron (CE) signal and of the main expected background.

Backgrounds can be grouped into two categories:

- Physical backgrounds that are common to all the conversion electron experiments and originate from the interactions of pions or muons in the ST. They include: muon decaying in orbit (DIO) and radiative nuclear captures of muons (RMC) and pions (RPC) and cosmic rays induced background.

- Instrumental backgrounds that are specific of Mu2e experiment and are related to the transportation of muons or other spurious negative particles through the beam line. This category includes $\mu^{-}$and $\pi^{-}$decays in flight, beam electrons and antiprotons.

After backgrounds description, the selection of signal vs background will be presented.

Finally the background level corresponding to the same run conditions used for the last blessed estimate of sensitivity ("CD3" of July 2017, [52]) will be calculated. Numerical comparisons with the previous results will be presented after a dedicated discussion of the antiproton background, to which the entire Chapter 4 is dedicated since it represents one of the main subjects of this thesis.

\subsection{The CE signal}

As discussed in the preamble of Chap. 2 the nominal momentum of an electron produced by the muon conversion in the $\mathrm{Al}$ atom is $104.97 \mathrm{MeV} / \mathrm{c}$. The monochromaticty of the conversion electron is certainly one of the most powerful tools to identify it.

Nonetheless it cannot be taken as strictly monochromatic when theoretical corrections and experimental resolutions are considered. Fig. 3.1 shows the spectrum after the radiative corrections. It can be described by the formula (normalized to the total free-muon decay width $\left.\Gamma_{\mu}\right)[53]$ :

$$
\frac{1}{\Gamma_{\mu}} \frac{d \Gamma}{d E_{e}}=\frac{\alpha}{2 \pi m_{\mu}}\left[\left(\ln \frac{4 E_{e}^{2}}{m_{e}^{2}}-2\right) \frac{E_{e}^{2}+m_{\mu}^{2}}{m_{\mu}\left(m_{\mu}-E_{e}\right)}+P\left(E_{e}\right)\right]
$$

where $\mathrm{P}\left(E_{e}\right)$ is polynomial factor dependent on the parameterization of the "soft" and "hard" photons. 

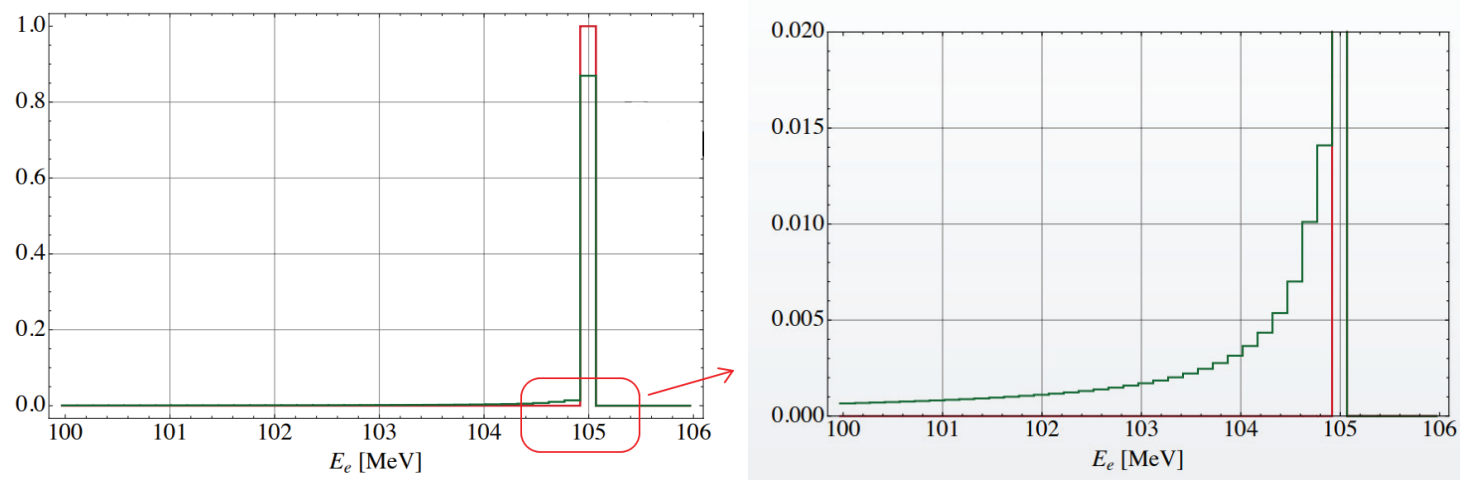

Figure 3.1: Effect of the radiative corrections on the monochromatic CE energy. The red bin contains events produced at the monochromatic energy of $104.97 \mathrm{MeV} / \mathrm{c}$, the green histogram is instead filled with the modified spectrum of Eq. 3.1.

In addition, the energy losses by the the electron in the material encountered before entering the tracker and the resolution of the track momentum (Fig. 2.18) make the reconstructed momentum distribution even broader.

To study the reconstructed momentum distribution for CEs, a sample of $10^{6}$ electrons is isotropically generated in the ST following Eq. 3.1. The starting positions have been obtained by a dedicated simulation of the muon beam from its production to the ST. Conversion electrons are then propagated through the tracker and the calorimeter: only the events producing at least 10 straw hits are reconstructed.

Track reconstruction is carried out using a logic OR between the tracker-only and the CalPatRec pattern recognition ${ }^{1}$. Then, in both cases, the track fit is performed with the TrkCaloHit method (2.6).

At the end of the simulation, $3.3 \cdot 10^{5}$ events have been successfully reconstructed and their momentum distribution is shown by the blue curve in Fig. 3.2. The product between the acceptance and the reconstruction efficiency is then estimated to be $\simeq 33 \%$. The plot compares the shifted and smeared distribution (blue curve) as it appears after propagation and reconstruction with the $\mathrm{CE}$ momentum under the monochromatic hypothesis (vertical red bar). The shift towards lower momenta is a direct consequence of the energy-loss inside the tracker, whose fluctuations contribute to enhance the left tail more than the right one. Note that the red filled curve represents the state of the distribution after the application of the signal selection cuts that will be described in Sec. 3.7.

\subsection{Cosmic Ray Background}

The cosmic ray (CR) induced background has been the limiting factor for the past conversion experiments and is one of the hardest challenges for the Mu2e experiment. It involves a multitude of different processes that can be a potential source of background for CEs: muons interactions in the ST, electrons entering the DS from TS hole, muons decays, neutral hadrons interacting in the calorimeter that produce upgoing particles then reflected by the magnetic mirror, etc...

\footnotetext{
${ }^{1}$ This takes all the advantages of the calorimeter-driven procedure but, in the meanwhile, is protected against its possible inefficiencies.
} 


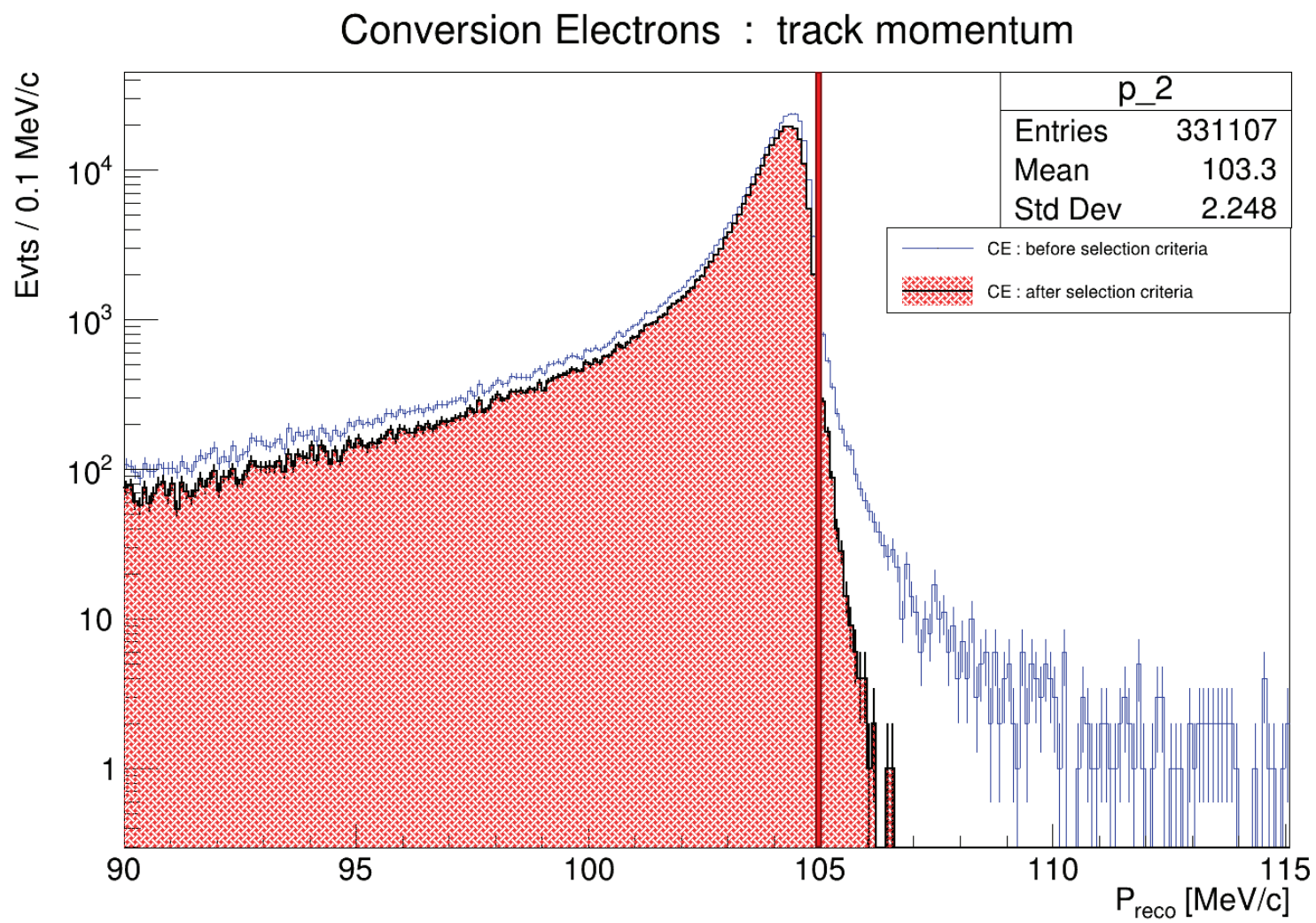

Figure 3.2: Reconstructed momentum for a dedicated simulation of $10^{6} \mathrm{CE}$ before (blue curve) and after (ref filled region) the selection criteria described in 3.7. The signal momentum in the monochromatic assumption is also shown in red.

CR background has unique features that make it quite different from all the others:

- it simply scales with the Mu2e effective data acquisition time;

- it is not related to the muon beam intensity so that its ratio to the CE signal becomes more unfavorable if the proton beam intensity or its stopped muon yield ${ }^{2}$ decreases;

- it is not influenced by the TS geometry or by the absorber material before the DS;

- differently from prompt backgrounds, it is not heavily suppressed by the live window time cut: it is just proportional to its width.

- its momentum distribution is nearly flat in the signal region so that also a momentum selection cannot get rid of it.

Due to all these reasons, a specific detector, the Cosmic Ray Veto (CRV), has been designed to suppress cosmic background.

Given the high flux of cosmic particles, the extension of the region to be studied and the abundance of even rare processes that may result in a non negligible background, the cosmic ray simulation is the most complex and computing resources demanding in Mu2e.

Before 2018 ([37], [52]), cosmic simulations were performed by using the Daya Bay (DBY) code [54], a generator considering only muon showers from primary cosmic protons.

\footnotetext{
${ }^{2}$ The number of muons stopped in the ST per proton on target.
} 
The energy spectrum and angular distribution are based on a modified version ${ }^{3}$ of Gaisser's formula [55] for the atmospheric muon intensity (Fig. 3.3):

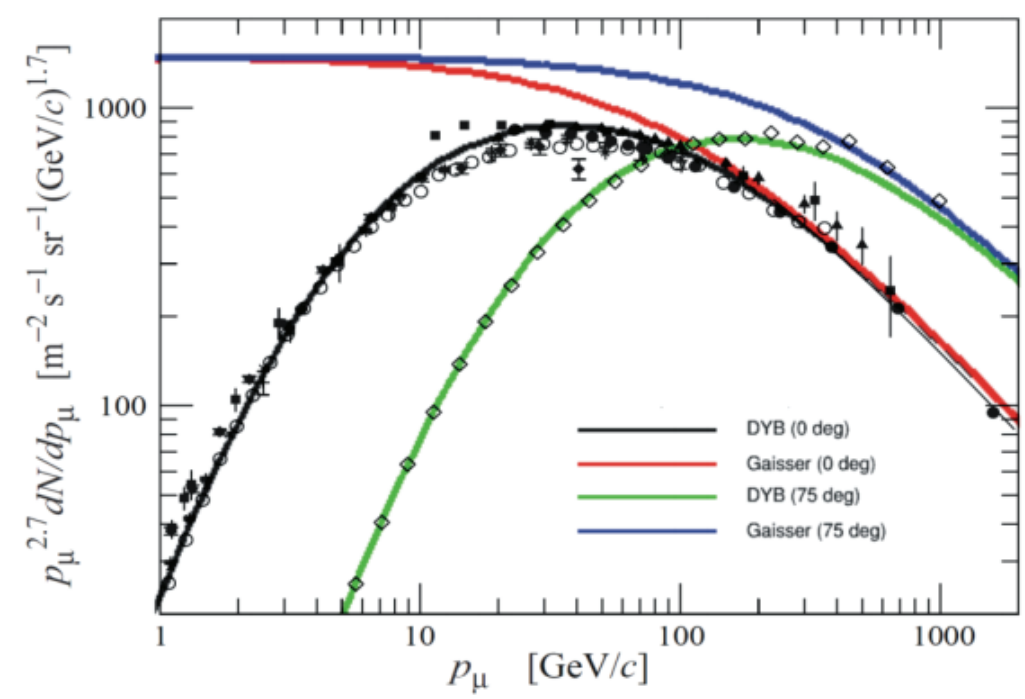

Figure 3.3: Differential intensity of the cosmic ray muons at two different angles, following the original Gaisser formula and its modified version implemented in the Daya Bay and CRY code.

$\frac{d R}{d A_{p} \cdot d \Omega \cdot d E_{\mu}}=\frac{0.14 E_{\mu}^{-2.7}}{c m^{2} s \operatorname{sr} G e V}\left(1+\frac{3.64 G e V}{E_{\mu}\left(\cos \theta^{*}\right)^{1.29}}\right)^{-2.7}\left(\frac{1}{1+\frac{1.1 E_{\mu} \cos \theta^{*}}{115 G e V}}+\frac{0.054}{1+\frac{1.1 E_{\mu} \cos \theta^{*}}{850 G e V}}\right)$

with:

$$
\cos \theta^{*}=\sqrt{\frac{(\cos \theta)^{2}+P_{1}^{2}+P_{2}(\cos \theta)^{P_{3}}+P_{4}(\cos \theta)^{P_{5}}}{1+P_{1}^{2}+P_{2}^{2}+P_{4}}}
$$

where $P_{1}=0.102573, P_{2}=-0.068287, P_{3}=0.958633, P_{4}=0.0407253, P_{5}=0.817285, \theta$ is the zenith angle, $A_{p}$ the production area, $E_{\mu}$ the energy of the generated cosmic muon.

More refined Monte Carlo productions have recently been obtained using the CRY generator [56] (and named the "CRY3 dataset"), which uses the same muon spectrum but also includes hadronic showers and allows to study the background induced by neutrons or photons.

In CRY3 cosmic particles are generated uniformly in a plane placed at the top of the Mu2e hall. These points are used as origin for the cosmic particles to be propagated to the DS.

As soon as they hit the CRV (if so) the are splitted into two different datasets, based on total energy deposited in the CRV, called: CosmicsHi ( $>14 \mathrm{MeV}$ ) and CosmicsLo ( $<14 \mathrm{MeV})$.

One of the critical parameters of the simulation is the light yield of the CRV scintillators. Due to the aging effect of the CRV scintillators in the hits recording, the Light Yield (LY) is expected to decrease by $8.7 \%$ per year.

Cosmic, no matter if they cross the CRV or not, are propagated through the DS ${ }^{4}$ where

\footnotetext{
${ }^{3}$ Modified to match the low-energy data in Fig. 3.3.

${ }^{4}$ It is possible to use each impact point on the CRV as starting point of multiple events to increase the final statistics.
} 
they eventually produce hits in the tracker or in the calorimeter. The same reconstruction procedure as CEs follows.

CRY3 dataset represents the highest-statistics simulation of the cosmic ray backgrounds currently available for Mu2e. It has gathered a total event statistics corresponding to a data acquisition time of $3.83 \cdot 10^{8} \mathrm{~s}$ (155 years) for CosmicsLo and $1.28 \cdot 10^{7} \mathrm{~s}$ (5.2 years) for CosmicsHi.

Fig. 3.4 shows the momentum distribution of all the reconstructed tracks (positive and negative particles) for both $L o$ and $H i$ datasets with no track selection nor CRV veto applied.
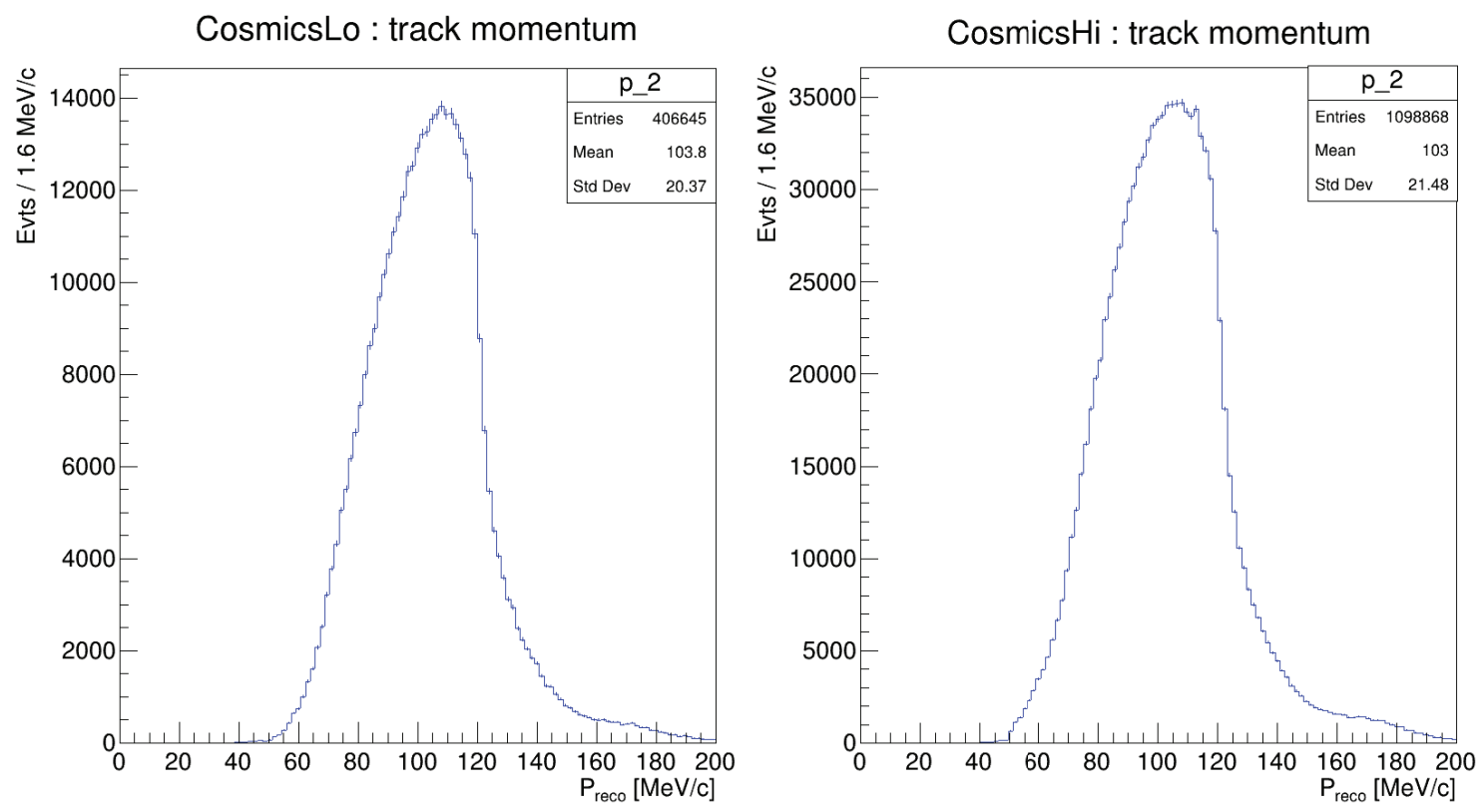

Figure 3.4: Reconstructed momentum distributions of particles originating from cosmic events. Neither the selection cuts and the CRV suppression are applied yet.

Most likely, CR tracks are not compatible with an event coming from the Stopping Target. One strategy to suppress them will be to look at two geometric parameters of the reconstructed track: the impact parameter and the momentum inclination with respect to the vertical axis.

\subsection{Decays in orbit (DIO)}

The muon decay-in-orbit (DIO) $\mu^{-} \rightarrow e^{-} \bar{\nu}_{e} \nu_{\mu}$ is, together with the muon nuclear capture, the main process contributing to the decay of a muonic atom. In the case of the Mu2e Al target the branching ratio for this process is $39.1 \%$. The time distribution for these decays is the one of the $\mathrm{Al}$ muonic atom decays (an exponential with $\tau=864 \mathrm{~ns}$ ) and is then the same of the one of the CE. The electron produced in the final state also has the same starting point distribution in the ST of the CE produced by muon conversion. As a consequence, any time or geometric selection do not help rejecting this background.

Fortunately the DIO momentum spectrum (Fig. 1.10) is much different from the CE one. 
The critical part of the differential spectrum is the tail for $E_{D I O}>85 \mathrm{MeV} / \mathrm{c}$, that is well described by a polynomial in $\Delta E=E_{D I O}-E_{C E}$ (from Czarnecki, Szafron [57] and [58]):

$$
\frac{1}{\Gamma_{\mu}} \frac{d \Gamma}{d E_{e}}=\frac{10^{-4}}{m_{\mu}}\left[\left(1.44 \Delta^{0.023}-0.22\right) \Delta^{5}+0.167 \Delta^{6}-0.767 \Delta^{7}+17.3 \Delta^{8}\right], \quad \Delta=\frac{\Delta E}{m_{\mu}}
$$

The term $\propto \Delta E^{5}$ accounts for $>80 \%$ of the events in the region above $103 \mathrm{MeV} / \mathrm{c}$, where the $\mathrm{CE}$ signal is searched for. For this reason the radiative corrections have been calculated only for this term [59].

DIO background are studied generating electrons with a flat and isotropic momentum distribution and an initial position and time according to simulated muon stops in the ST. The flat distribution is then weighted to obtain the DIO spectrum. This weighting technique allows to disentangle the generation from the preferred theoretical model and enhances the statistics in the high momentum region that is the most critical for the $\mathrm{CE}$ background.

The accuracy of the momentum reconstruction is the most important tool to reject DIOs and it is of pivotal importance to reproduce at best the experimental condition in which the tracker will work. In order to have a more realistic simulation of the track pattern recognition and reconstruction, the pileup events coming from the beam simulation are randomly superimposed to the DIO events (we will refer to it with the name "background mixing" ${ }^{5}$. In the past, this has revealed to be crucial but, at the time of writing, updated simulations implementing this procedures are not yet available. The best procedure is then to get the reconstructed momentum spectrum from a previous simulation (2018) and use the latest one without mixing, but with an improved track selection (2020) to set the normalization.

Fig. 3.5 shows the track reconstruction efficiency, including the geometrical acceptance, as function of the generated momentum obtained by $\sim 2 \cdot 10^{7}$ electrons generated with a flat distribution between $75 \mathrm{MeV} / \mathrm{c}$ and $110 \mathrm{MeV} / \mathrm{c}$ with superposed background mixing: the acceptance is $\sim 30 \%$ at $106 \mathrm{MeV} / \mathrm{c}$ and rapidly decreases while going down to 75 $\mathrm{MeV} / \mathrm{c}$.

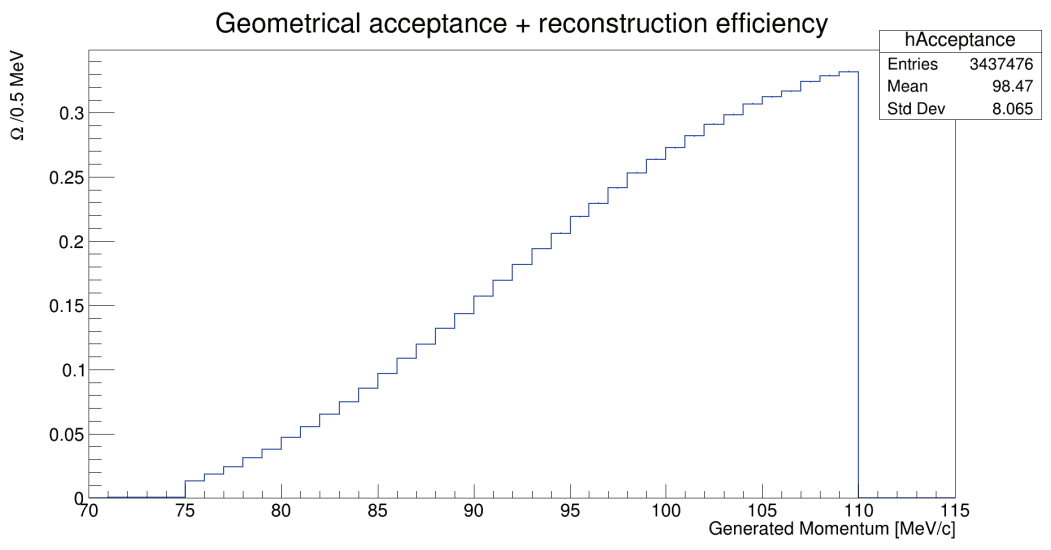

Figure 3.5: Bin acceptance for flat produced electrons in the range $[75,110] \mathrm{MeV} / \mathrm{c}$ (blue curve). Every bin contains the ratio between the reconstructed events with original momentum in that bin and the number of generated events in the same bin.

\footnotetext{
${ }^{5}$ The actual number of pileup events were determined by the proton beam intensity and its fluctuations.
} 
The reconstructed momentum spectrum of DIO events can be obtained applying the proper weight to each event. If the event belongs to the generated momentum bin $[\mathrm{p}, \mathrm{p}+$ $\Delta \mathrm{p}]$ of width $\Delta p$, its weight will be:

$$
w=\frac{N_{C S}(p, p+\Delta p)}{N_{g e n}(p, p+\Delta p)}
$$

where $N_{\text {gen }}(p, p+\Delta p)$ is the number of events generated in the bin by the flat generation:

$$
N_{\text {gen }}(p, p+\Delta p)=\frac{N_{G e n}}{n_{\text {bins }}}=\frac{N_{G e n} \times \Delta p}{p_{\max }-p_{\min }}
$$

with $p_{\min }=75 \mathrm{MeV} / \mathrm{c}$ and $p_{\max }=110 \mathrm{MeV} / \mathrm{c}$, while $N_{C S}$ is the number of expected DIO events in the bin according to the Czarnecki-Szafron probability $\mathcal{P}_{C S}$ for a given number of protons on target $N_{P O T}$ :

$$
N_{C S}(p, p+\Delta p)=N_{P O T} \times \mathcal{P}(\mu \text { stops } \mid P O T) \times \mathcal{P}(D I O \mid \mu \text { stops }) \times \mathcal{P}_{C S}(p, p+\Delta p)
$$

where:

- $\mathcal{P}(\mu$ stops $\mid$ POT $)=0.0016$ is the muon stop probability per POT;

- $\mathcal{P}(D I O \mid \mu$ stops $)=0.391$ is the DIO branching ratio.

The DIO reconstructed momentum distribution, with the proper event weights, is shown in Fig. 3.6 both for th 2018 dataset with background mixing and the 2020 one without it. As it can be seen the impact of the mixing procedure is not negligible at this step: the spectrum peak is shifted towards lower momenta and the RMS of the distribution is larger. If we compare the left plot with the CS momentum spectrum of Fig. 1.10 we
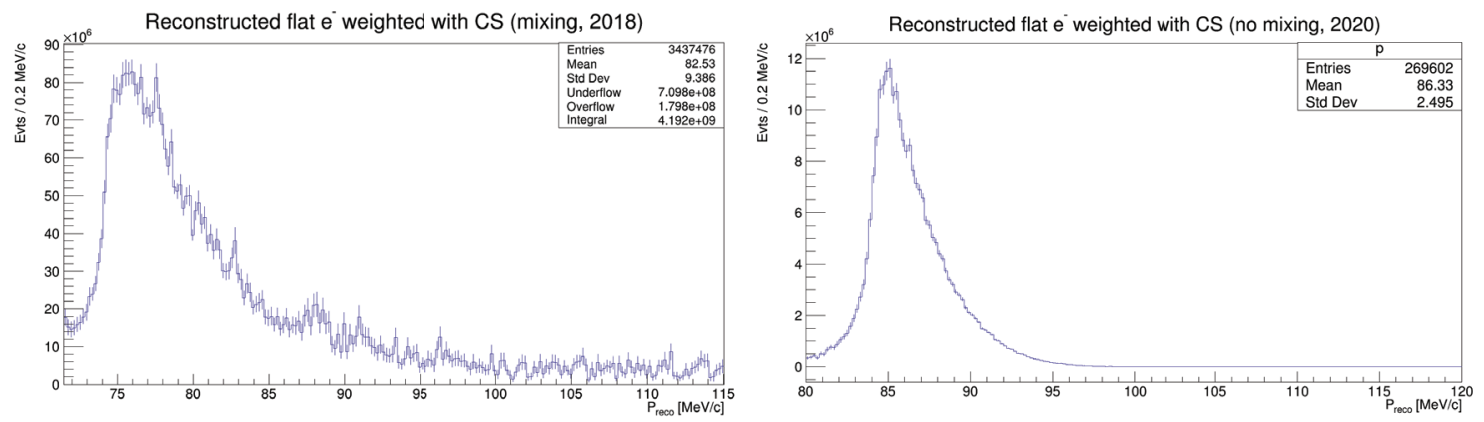

Figure 3.6: DIO reconstructed momentum distributions with (2018, left plot) and without (2020, right plot) background mixing. Both plots are normalized to $3.6 \cdot 10^{20}$ POT. The quality cuts have not yet been applied.

see that the huge right tail cannot be ascribed only to the DIO theoretical spectrum but is due the tail of the momentum resolution, which becomes also more relevant when the background mixing is applied. A requirement on the "quality" of the reconstructed track, together with other geometrical cuts, will be the key to eliminate this source and restore the CS shape. 


\subsection{Radiative pion captures (RPC)}

Radiative Pion Captures (RPC) have been presented in Sec. 2.2.2 as one of the main sources of background if not cured with an appropriate time window cut.

RPC background can arise from two different processes:

- external conversion: an on-shell ("real") photon emitted in the capture that then converts in an $e^{-} e^{+}$pair in the $\mathrm{ST}$ or in the detector;

- internal conversion: during the capture an off-shell ("virtual") photon is emitted and immediately converts to an $e^{-} e^{+}$pair. In this case there is no photon in the final state but one $e^{-}$and one $e^{+}$.

The probability of emitting a real photon is significantly higher than the probability to emit a virtual one ${ }^{6}$ ). However, the probability for a real photon to convert in the path from the ST to the detector is not so high and the final effect is that external and internal conversion processes produce nearly the same amount of electrons.

In addition, it is possible to distinguish between two components of the RPC background:

- in-time RPC: due to pions coming from protons belonging to the proton bunch. These events can be efficiently suppressed by a time window cut.

- out-of-time RPC: produced by out of bunch protons ${ }^{7}$ and able to elude the time window cut. Mu2e assumes a uniform time distribution for this events.

The relative importance of the two components depends on the extinction factor provided by the extinction system (Sec. 2.2.3): if this is $O\left(10^{-10}\right)$ they will contribute at the same level.

The RPC simulation consists of 5 stages. The first 3 stages are shared by internal and external RPC: they perform the propagation of pions from the Production Target to the Stopping Target and determine the time and position distribution of the $\pi^{-}$stopped in the ST. To increase the yield of stopped pions, their decay is disabled during this propagation. The information about the pion proper time is stored and is in the next stages used to calculate the weight corresponding to the event probability.

The fourth stage produces photons at the positions of pion stops in the ST. The same pion stop can be reused multiple times (resampling) to produce photons of different energy and momentum creating independent events.

The on-shell photon spectrum is taken from the "Bistirlich distribution" (Fig. 3.7) ${ }^{8}$ [60]:

$$
\frac{d N_{\gamma R P C}}{d E_{\gamma}}=\left(E_{\max }-E_{\gamma}\right)^{\alpha} e^{-\frac{\left(E_{\max }-\gamma E\right)}{\tau}}\left(c_{0}+c_{1} E_{\gamma}\right)
$$

With $E_{\max }=134.5 \mathrm{MeV}$ being the spectrum endpoint and $\alpha=1.299, \gamma=0.9289$, $\tau=9.397, c_{0}=0.04776$ and $c_{1}=-0.000326$ the fit parameters (energies are in $\mathrm{MeV}$ ).

\footnotetext{
${ }^{6}$ The internal conversion probability is at least a factor $\alpha$ lower than the external conversion one.

${ }^{7}$ Another source of out-of-time pions is given by the annihilation of slow antiprotons in the TS. This RPC component will be ascribed to the antiproton background and discussed in the next chapter.

${ }^{8}$ The RPC spectrum has actually been measured and modeled for the magnesium $(\mathrm{Mg})$ : as best available approximation it has been assumed to be the same for aluminum.
} 


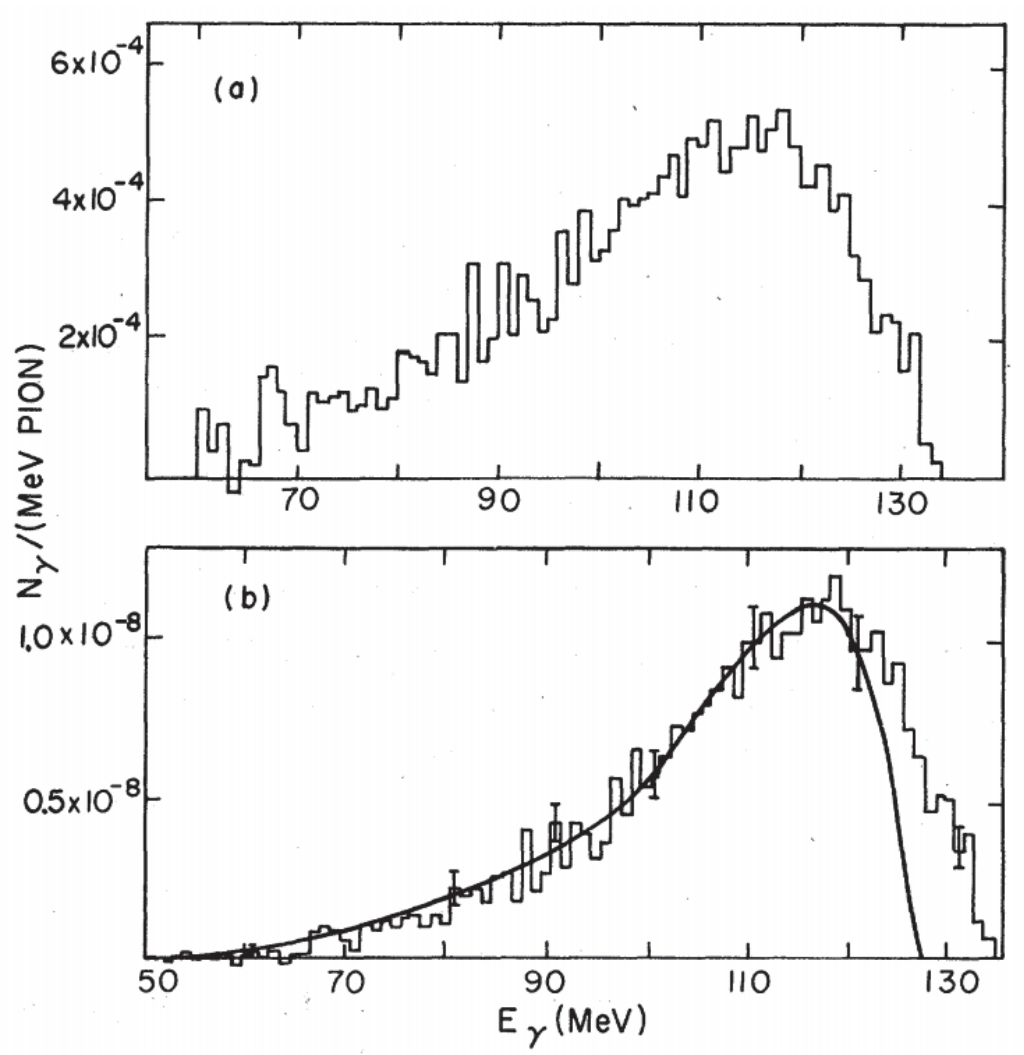

Figure 3.7: Energy spectrum of photons from radiative pion capture in magnesium. (TOP) Spectrum with efficiency divided out. (BOTTOM) Measured spectrum; solid line: Bistirlich pole-model predictions.

For the internal conversions, the virtual photon spectrum is not experimentally accessible and so the same Bistirlich formula is then taken as first approximation (the systematic uncertainty connected to this assumption will be discussed later ${ }^{9}$ ).

Since for virtual photons conversion is practically instantaneous, the $e^{-} e^{+}$pair is produced in the same position of the pion stop. The handling of the generation spectrum of the the $e^{-} e^{+}$pair is left to Geant4.

Events are then propagated through the tracker and the calorimeter and their tracks and energy deposits are reconstructed.

The number of reconstructed tracks for the 4 RPC components (internal or external, in-time or out-of-time) corresponding to $N_{P O T}$ protons on target can be calculated as:

$$
N_{R P C}=N_{P O T} \cdot \xi \cdot \frac{N_{\text {stop }}}{N_{P_{\text {OOT }}}} \cdot \frac{\sum_{\text {ston }} e^{-t_{p} / \tau_{\pi}}}{N_{\text {stop }}} \cdot f_{R P C} \cdot\left[\rho_{\gamma^{*} \rightarrow e^{+} e^{-}}\right] \cdot \frac{\sum_{\text {signal }} e^{-t_{p} / \tau_{\pi}}}{\sum_{\gamma \text { gen }} e^{-t_{p} / \tau_{\pi}}}
$$

where:

- $\xi$ is the extinction factor, that is taken equal to 1 for the in-time events and is required to be better than $10^{-10}$ for the out of time events [37]

\footnotetext{
${ }^{9} \mathrm{~A}$ possible future extension of this work might include a specific generator based on the theoretical argument presented in [61] and [62].
} 
- $\mathcal{P}_{\text {stop }}^{\pi(\tau=\infty)}=\frac{N_{\text {stop }}}{N_{P_{\text {OT }} \text { gen }}}=0.00248$ is the number of stopped pions per proton on target with pion decay turned off as obtained from the third stage of the simulation stages.

- $\mathcal{P}_{\text {surv }}^{\pi}=\frac{\sum_{\text {stops }} e^{-t_{p} / \tau_{\pi}}}{N_{\text {stop }}}$ is the correction due to the pions decay. Each stopped pion has a survival probability $e^{-t_{p} / \tau_{\pi}}$, where $t_{p}$ is the proper time the pion has traveled to reach the ST and $\tau_{\pi^{-}}=2,6 \cdot 10^{-8} \mathrm{~s}$ is the $\pi^{-}$decay time. The correction is given by the ratio between the sum of the pion stops survival probabilities and the total number of stops $N_{\text {stop }}$.

- $f_{R P C}=(2.15 \pm 0.20) \%$ is the $\mathrm{RPC}$ branching ratio on $\mathrm{Mg}{ }^{10}$ [63].

- $\rho_{\gamma^{*} \rightarrow e^{+} e^{-}}=0.00690 \pm 0.00038$ is the internal conversion probability [61], to be taken into account only for the calculation of the internal RPC. The uncertainty is a combination of values reported in experimental and theory papers [64].

- $\mathcal{P}\left(e^{-}\right.$reco. $\left.\mid \gamma\right)=\frac{\sum_{\text {signal }} e^{-t_{p} / \tau_{\pi}}}{\sum_{\gamma \text { gen }} e^{-t_{p} / \tau_{\pi}}}$ is the final factor that estimates the probability for a photon to produce a reconstructed electron track.

When the simulation is integrated with the background hits due to other particles coming with the beam (pileup) the results don't change significantly [64].

The momentum distribution of the reconstructed tracks is shown in Fig. 3.8, normalized to $3.6 \cdot 10^{20} \mathrm{POT}$, for both internal ( $\left.R P C i\right)$ and external ( $R P C e$ ) in-time RPC (always with no selection cuts at this step) simulations. As expected, the two contributions are very similar.
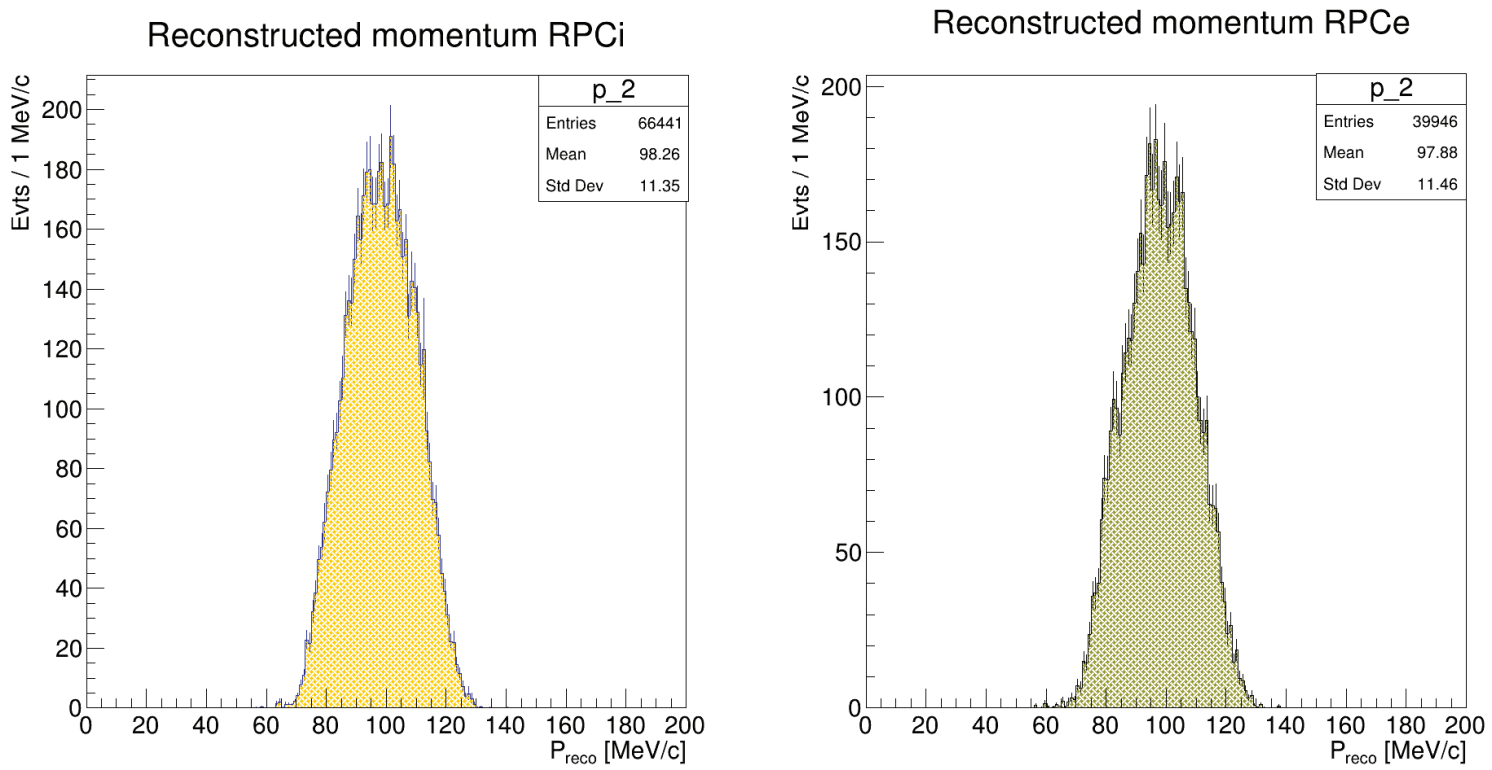

Figure 3.8: Reconstructed momentum distribution of the tracks originating from RPCs. The histograms are scaled to a run plan statistics of $3.6 \cdot 10^{20}$ POT.

\footnotetext{
${ }^{10} \mathrm{Again}$ the $\mathrm{Mg}$ value is taken as best approximation since the $\mathrm{Al}$ one is not available.
} 
Based on previous analyses ([64]), the contribution coming from the out-of-time RPCs is expected to equal the in-time one if an extinction factor of $O\left(10^{-10}\right)$ is assumed. The extinction system is actually foreseen to achieve a much better result: $\xi \sim 10^{-12}$. For this reason the out-of-time contribution will be neglected in the discussion.

The primary tool to suppress this background is the time dependence. Distribution for the track parameter $T_{0}$, i.e. the track time evaluated at the center of the tracker, is presented in Fig. 3.9. The internal and external contribution acts in the same way in this variable, so the plot is shown for the external contribution only.
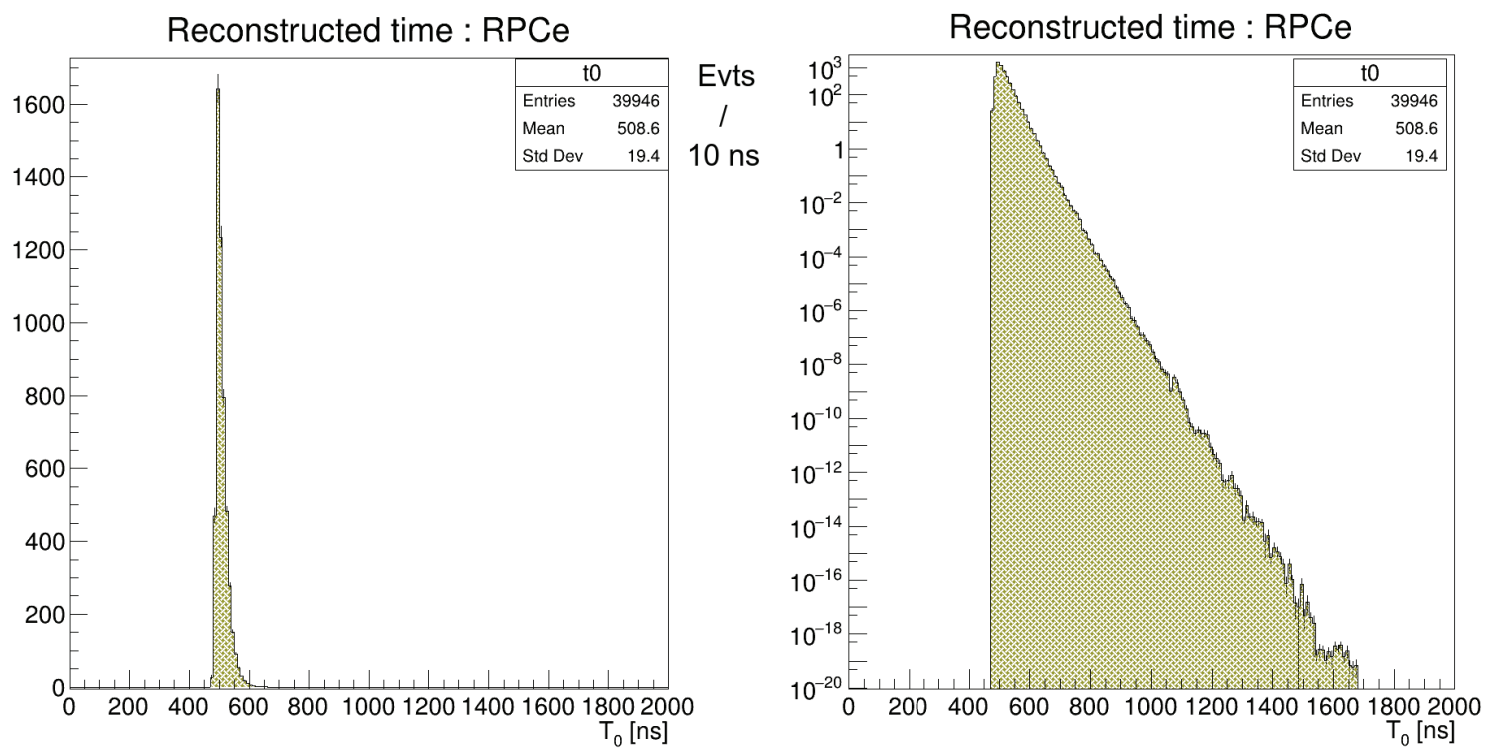

Figure 3.9: Distributions of the times in the center of the tracker for external RPC events. The plot is shown in both linear and logarithmic scale. Histograms are scaled to a run plan statistics of $3.6 \cdot 10^{20} \mathrm{POT}$.

\subsection{Instrumental Backgrounds}

The main background sensitive to the beam line design comes from the antiprotons produced in the Production Target and will be extensively discussed in the next chapter.

Other processes that give a much less relevant contribution to the $\mathrm{CE}$ background are:

- beam electrons: the multitude of $\pi^{0} \mathrm{~s}$, produced in primary proton interactions in the PT, immediately decay $\left(\tau_{\pi^{0}} \simeq 8.4 \cdot 10^{-8} \mathrm{~ns}\right)$ producing photons and electrons-positron pairs (Dalitz decays). Photons cannot be bent by the TS magnetic field and thus are not a direct background source. The electrons produced in their conversion can instead reach the DS and be scattered off the ST, mimicking a CE in the detectors. Their propagation is very fast and they generally reach the DS in the first $200 \mathrm{~ns}$. This makes in-time beam electrons completely negligible in the signal time window. An out-of-time component due to out of bunch protons will be in any case suppressed by the extinction factor to a value at least one order of magnitude lower than the out-of-time RPCs [65];

- Muons and pions decay in flight: $\mu^{-}$and $\pi^{-}$passing the TS can decay in flight (DIF) and produce electrons. Simulations made at the time of the Technical Design Report 
show that those electrons do not reach detectors later than 500 ns after the proton pulse arrival and are suppressed to a negligible level by the time window cut.

\subsection{Radiative muon captures (RMC)}

The ordinary muon nuclear capture $\left(\mu^{-27} \mathrm{Al} \rightarrow \nu_{\mu}{ }^{27} \mathrm{Mg}^{*}\right)$ is definitely not a background source for the Mu2e experiment. But a variation of the process is possible, in which an initial state radiation or a final state radiation produces a photon (real or virtual):

$$
\mu^{-27} \mathrm{Al} \rightarrow \gamma \nu_{\mu}{ }^{27} \mathrm{Mg}^{*}
$$

that can possibly convert and create an electron.

The energy spectrum of RMC photons in aluminum has been measured by the TRIUMF experiment [66] (Fig. 3.10, right) and modeled by the so called "closure approximation":

$$
\frac{d N_{\gamma R M C}}{d E_{\gamma}}=\frac{e^{2} k_{\max }^{2}}{\pi m_{\mu}^{2}} \cdot \frac{2 Z}{A} \cdot\left(1-2 x+2 x^{2}\right) \cdot x \cdot(1-x)^{2}, \quad x=\frac{E_{\gamma}}{k_{\max }}
$$

where A,Z, are the mass and atomic number of the considered nucleus.

The key parameter is the maximum photon energy allowed by the kinematic: $k_{\max }$. The theoretical spectrum is shown in the left side plot of Fig. 3.10 for different values of $k_{\max }$. The yellow curve corresponds to $k_{\max }=101.853 \mathrm{MeV}$, which is the kinematic limit
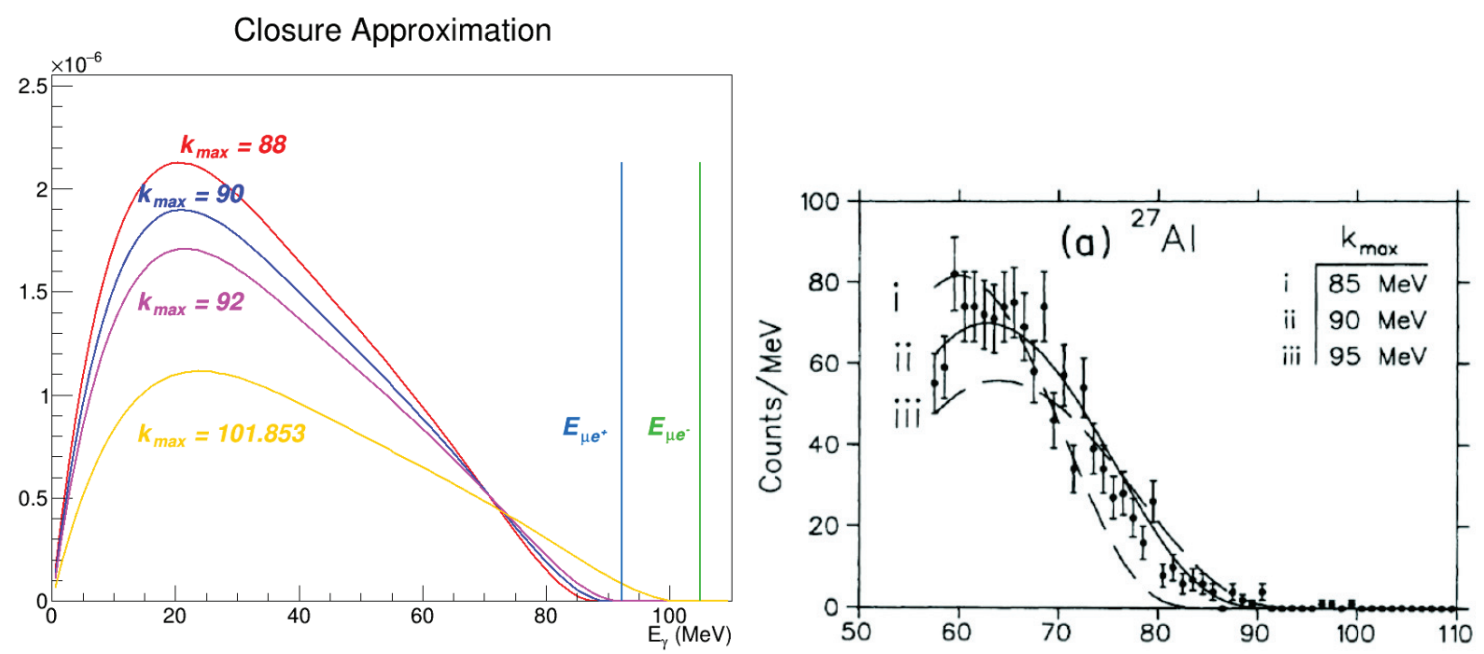

Figure 3.10: (LEFT) RMC photon spectrum modeled with the closure approximation, for different values of the kinematic endpoint $k_{\max }$. The monochromatic energy for a conversion electron or position are also shown as vertical lines. (RIGHT) RMC photon spectrum modeled with the closure approximation superposed to the TRIUMF data for aluminum in the range $>57 \mathrm{MeV}$.

obtained by the naive formula:

$$
k_{\max }=m_{\mu}-B_{\mu}-R_{N}-\Delta M
$$

where $m_{\mu}$ is the muon mass, $B_{\mu}$ the binding energy of the atom, $R_{N}$ the nucleus recoil and $\Delta M$ the mass difference between the original and the daughter nucleus. This formula neglects higher order nuclear effects and its prediction is in disagreement with the spectrum measured by TRIUMF. 
A more accurate calculation is being preformed by a group of theorists in contact with the Mu2e collaboration.

If the $k_{\max }$ value obtained by the best fit of TRIUMF data is used, the blue spectrum in Fig. 3.10 is obtained. The magenta and red curves in the same picture represent its $\simeq 1 \sigma$ variation using the uncertainty quoted from the original paper. The TRIUMF uncertainty has in fact been found to be systematically overestimated. A detailed study ([67]) has revealed that it most likely needs to be rescaled from $1.8 \mathrm{MeV} \rightarrow 0.5 \mathrm{MeV}$ :

$k_{\max }=(90.1 \pm 0.5) \mathrm{MeV}$

The probability to have a photon with energy $E>57 \mathrm{MeV}$ has been also been measured by TRIUMF:

$$
B R_{\text {ext } R M C}>57 \mathrm{MeV}=(1.43 \pm 0.13) \cdot 10^{-5}
$$

This low probability and the kinematic limit very far from the CE momentum make the RMC background completely negligible ${ }^{11}$.

\subsection{Variables for CE selection}

Now that the characteristics of the CE signal and of the main possible backgrounds have been presented, it is possible to identify the variables that can efficiently select the CEs.

\subsubsection{Geometric cuts}

- Time direction: the track must be compatible with a particle moving downwards from the tracker to the calorimeter. This can be checked looking at the times ordering of the hits of the fitted track and of the calorimeter cluster. This request is mostly intended to reject the events originated in the calorimeter by cosmic interactions.

- Sign of the charge: it must be negative as expected for electrons. The track must have a negative curvature in the DS magnetic field (anticlockwise rotation for downgoing particles).

- Impact parameter: $d_{0}$, defined as the distance of closest approach, in the DS transversal plane, of the reconstructed track to the ST. A negative sign is assigned if the projected circle contains the ST. Requiring the compatibility with a track originating from the Stopping Target, the highest populated region of the cosmics distribution in this variable can be filtered out (as shown in the left plot of Fig. 3.11).

- Pitch angle: $\theta$, this is the momentum inclination with respect to the vertical. This also has a strong impact on cosmic selection (Fig. 3.11, right): vertical cosmics have $\theta \simeq 0$ while the one entering from the TS hole are nearly horizontal $\left(\theta \simeq \frac{\pi}{2}\right)$. The cut on the high angles also helps suppressing pions and muon decays in flight.

\footnotetext{
${ }^{11}$ The same is not true if one wants to look for the $\mu^{-} \rightarrow e^{+}$conversion where the expected $e^{+}$conversion energy is $92.32 \mathrm{MeV}$ : in this case RMC becomes one of the main backgrounds.
} 

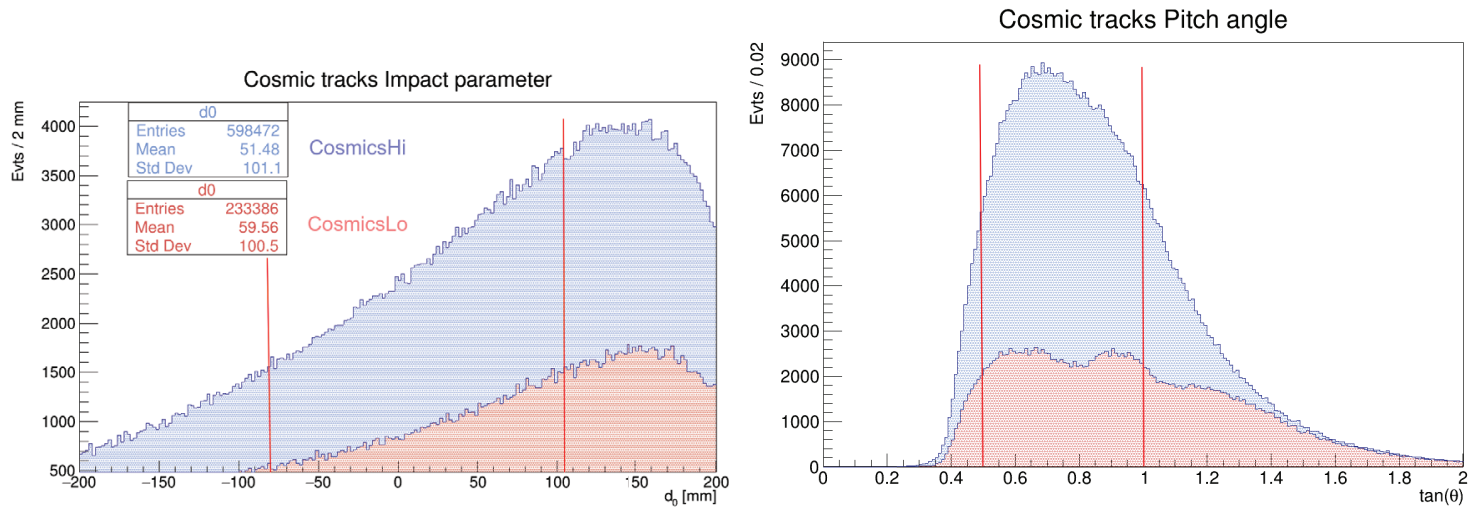

Figure 3.11: Geometric cuts on the impact parameter and pitch angle distribution for cosmic events reconstructed in the CRY3 simulation. The accepted regions are the ones between the red vertical lines.

- Maximum helix radial distance (from the ST): it must be such that the helix (with radius $\mathrm{R}$ ) does not intersect the Outer Proton Absorber and its projection on the transversal plane must be confined within the radius of the tracker.

A graphic representation of the small impact of these last three geometric cuts on CEs is shown in Fig. 3.12.
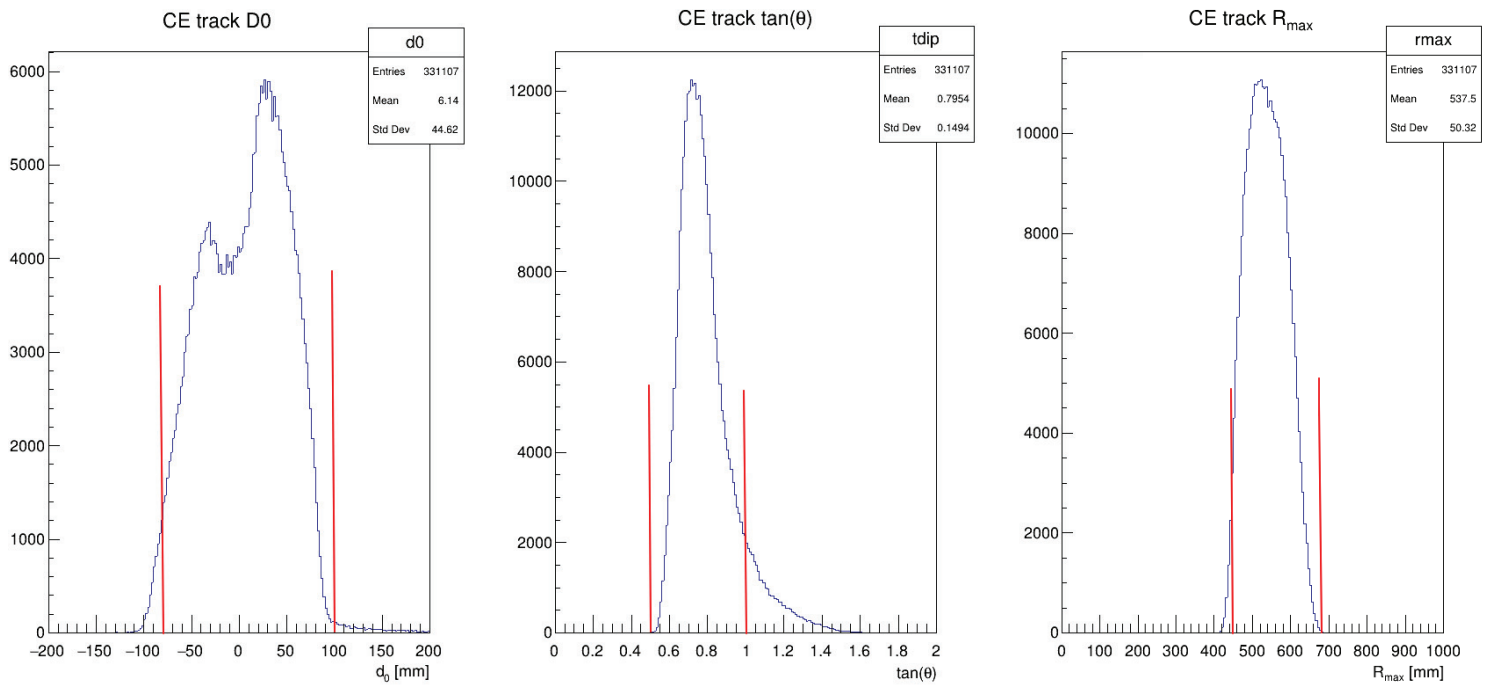

Figure 3.12: Geometrical requirements on the reconstructed tracks. The plots shows (from the left to right) the impact parameter $d_{0}$, the tangent of the pitch angle and the helix radius distribution for reconstructed CE tracks.

\subsubsection{Track quality}

A big part of the reconstructed momentum smearing observed for DIOs and its dangerous right tail is due to bad track reconstruction. Some quality cuts on the tracks can help to reduce the momentum overlap between CEs and DIOs without significantly decreasing the efficiency on the signal. The variables used to select good quality tracks 
are: the fit momentum error, the number of hits used by the fit, the fraction of observed hits compared to expected, the fraction of hits to which it was not possible to assign a drift distance, the fraction of hits not rejected by the Kalman filter, the fit time error and the $\chi^{2}$ consistency.

This set of variables is used to train an Artificial Neural Network (ANN) classifier [68] that maximizes the separation between tracks with "good" momentum reconstruction $\left(\left|p_{\text {reco }}-p_{\text {gen }}\right|<500 \mathrm{keV} / \mathrm{c}\right)$ and tracks with "bad" reconstructed momentum $\left(\left|p_{\text {reco }}-p_{\text {gen }}\right|>\right.$ $1 \mathrm{MeV} / \mathrm{c})^{12}$. The selection is then made with a box cut on the ANN output, called TrkQual.

Fig. 3.13 shows the effect of the track quality cut on flat generated electrons with a reconstructed momentum $95<p<105 \mathrm{MeV} / \mathrm{c}$ : the suppression of the high momentum tail is evident. The distribution is well fitted by a double-sided Crystal Ball function with

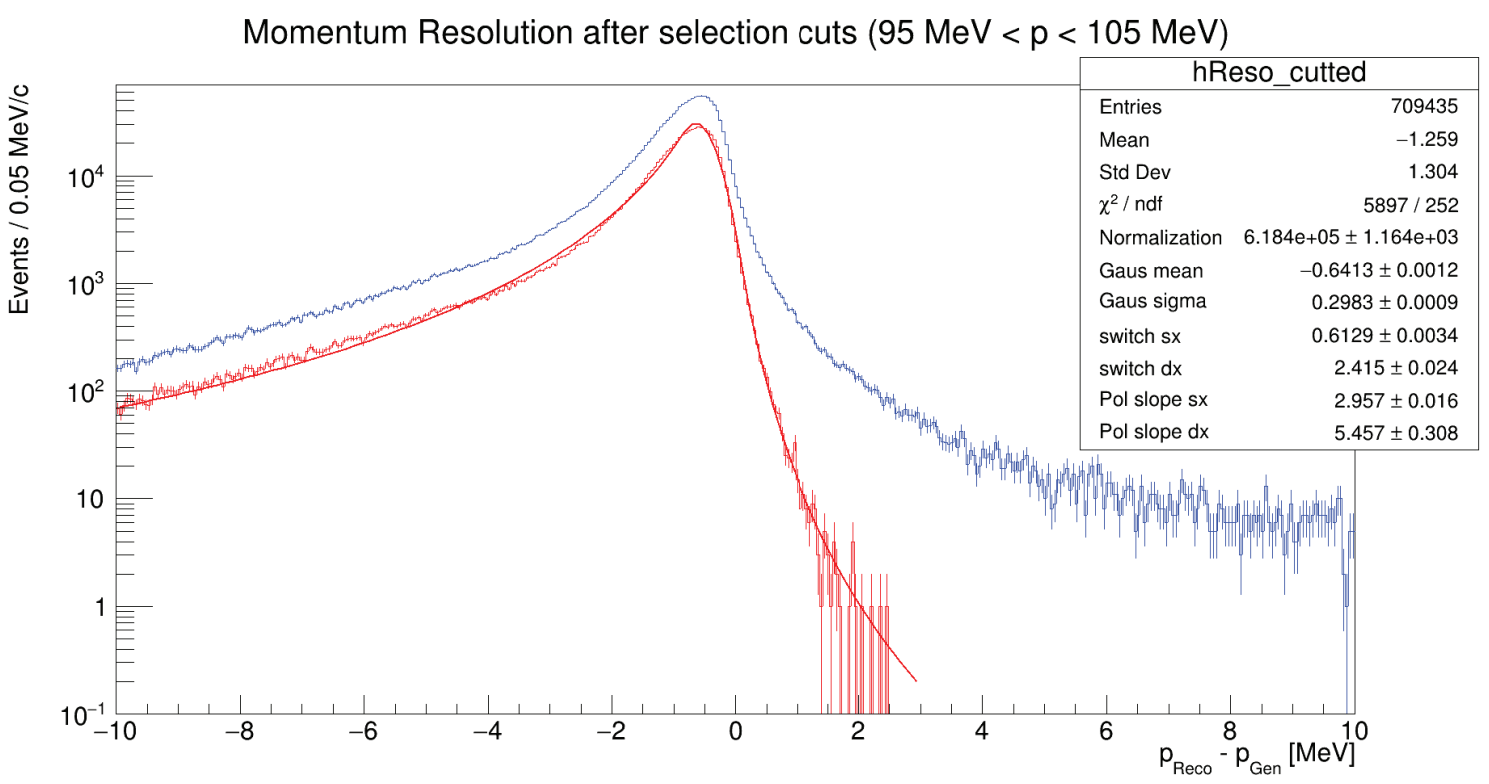

Figure 3.13: Distribution of the events in the momentum difference $p_{\text {Reco }}-p_{\text {Gen }}$ variable. The plot is shown for a subset of the total events between $95 \mathrm{MeV} / \mathrm{c}<p_{\text {Reco }}<105 \mathrm{MeV} / \mathrm{c}$ and before (blue histogram) and after (red histogram) quality cuts.

a $\mathrm{FWHM}=802 \mathrm{keV} / \mathrm{c}$. The momentum resolution is dominated by three effects: energy loss fluctuations in the ST, energy loss fluctuations in the Inner Proton Absorber and the precision of the track reconstruction algorithm. All the three process have the same relevance: the result can be seen as the sum in quadrature of three equal contributions of $\sim 500 \mathrm{keV} / \mathrm{c}$ each.

When the track quality cuts are applied to the DIO events, the spectrum is modified as shown in Fig. 3.14 : the tail in the signal region $(p \sim 105 \mathrm{MeV} / \mathrm{c})$ has been highly suppressed.

The efficiency of the track quality cuts on reconstructed tracks is $\simeq 90 \%$.

\footnotetext{
${ }^{12}$ This definition of 'good' and 'bad' tracks is very effective in suppressing the right-side tail of the momentum resolution and therefore the DIO background [69].
} 


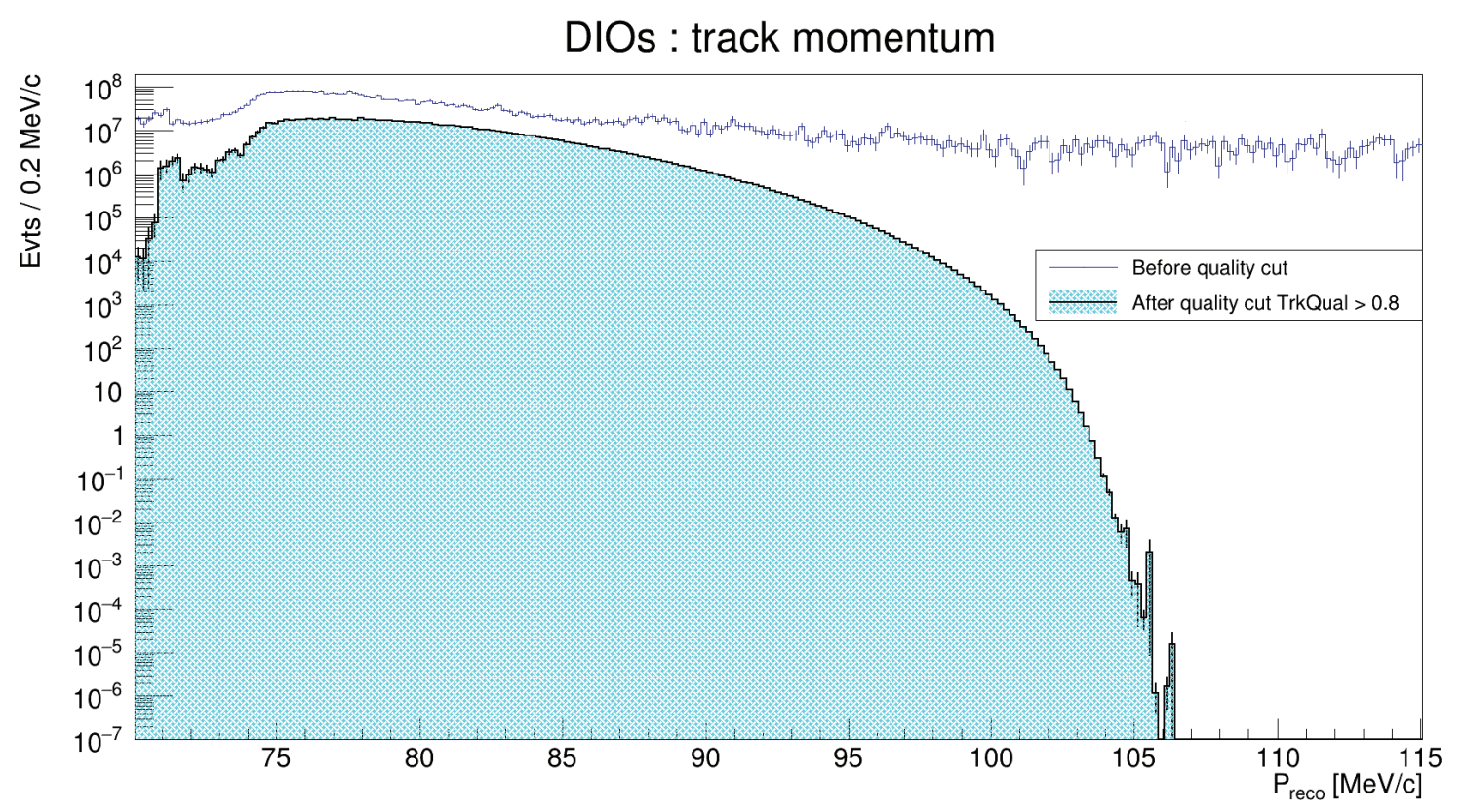

Figure 3.14: Suppression of the right side tail of the reconstructed DIO momentum distribution. The non-filled blue curve represent the reconstructed and weighted DIO events (exactly the $\log$ scale version of Fig. 3.6). The azure-filled histogram shows the same distribution after the selection on the track quality variable (TrkQual $>0.8)$. Both plots are normalized to $3.6 \cdot 10^{20}$ POT.

\subsubsection{A simple Particle Identification (PID)}

An important source of contamination among the reconstructed tracks is given by muons having a momentum compatible with CEs. The above track quality selection cannot distinguish between muon and electron events and thus a simple particle identification (PID) selection is implemented. It consists of two cuts:

- Energy-momentum matching: the E/p ratio between the cluster energy measured by the calorimeter and track momentum measured by the tracker must be compatible with that of an electron. As the energy deposited in the calorimeter by a muon corresponds to its kinetic energy, this is in general significantly less than the muon momentum. $\mathrm{E} / \mathrm{p}$ is generally lower than 1 also for an electron, mainly because of the incomplete containment of the shower (energy leakage) but it is actually much closer to 1 , so that a cut $E / P>0.6$ is able to reject most of the muons while keeping the $92 \%$ of the electrons.

- Time of flight: $\mathrm{a} \sim 105 \mathrm{MeV} / \mathrm{c}$ muon has a $\beta \sim 0.7$ that is quite different from the $\beta \sim 1$ of the $\mathrm{CE}$. The time difference between the cluster time measured by the calorimeter and the time at the center of the tracker obtained by the track fit has an excellent separation power on $\sim 105 \mathrm{MeV} / \mathrm{c}$ electrons and muons.

Both selections are driven by the muon contamination in the tracks originating from cosmics, as shown in both plots of Fig. 3.15.

These cuts in principle introduce a $\sim 8 \%$ reduction of the CE efficiency. A PID selection based on a multivariate technique (a Boosted Decision Tree (BDT) classifier), has been demonstrated to provide the same background rejection without affecting CEs ([70]). To 

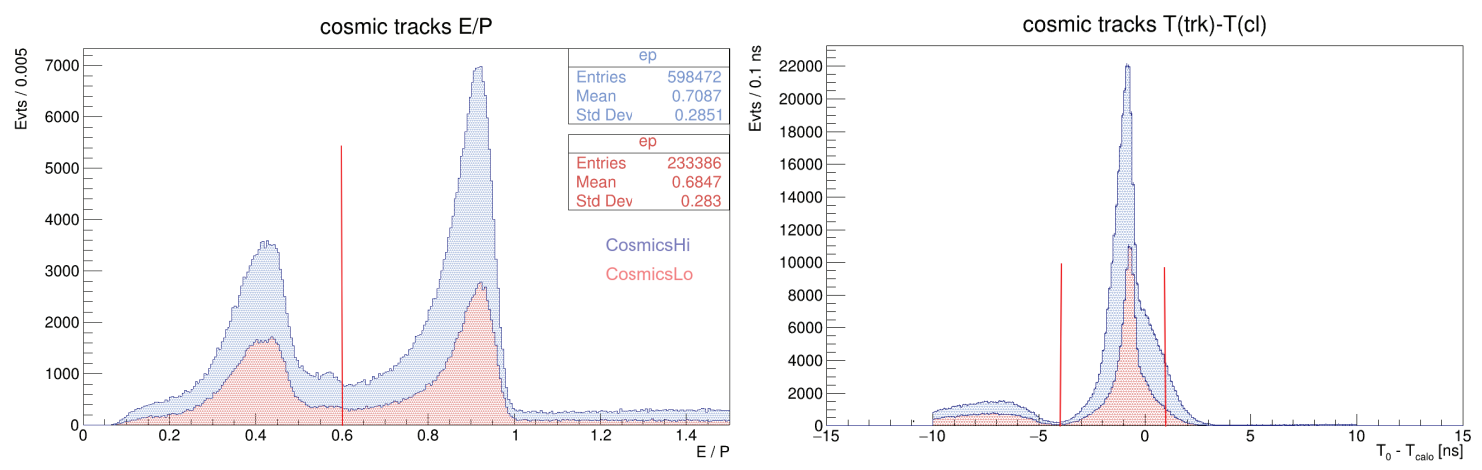

Figure 3.15: PID selection shown on the cosmic distributions. (LEFT) E/p distribution for reconstructed cosmic tracks in the CRY3 simulation. The region $\mathrm{E} / \mathrm{p}>0.6$ is the allowed one. (RIGHT) Same sample but the distribution is shown in the time of flight variable. The selected region is $-3.5<\Delta T(\mathrm{~ns})<1$.

emulate the performances of this classifier, not yet available in the Mu2e software package, the PID cuts described above have been applied only to the backgrounds and not to CE.

In order to suppress the "unreducible" cosmic background given by the $\sim 105 \mathrm{MeV} / \mathrm{c}$ electrons produced in the ST by cosmic interactions, the Cosmic Ray Veto must be applied. The actual implementation just requires reconstructed events in the CRY3 sample not to be associated to a triple coincidence and energy deposit in the CRV $\left(N_{C R V \text { clusters }}=0\right.$, see Sec. 2.8). Mu2e will ultimately required the track in all channels to be isolated in time with respect to a CRV detection, i.e. no CRV cluster in $\left[T_{\text {track }}-120 \mathrm{~ns}, T_{\text {track }}+50 \mathrm{~ns}\right]$. The event suppression in the cosmic datasets improves by a factor $\simeq 3 \cdot 10^{3}$ for CosmicsHi and $\simeq 30$ for CosmicsLo.

\subsubsection{Optimized cuts and numerical values}

The two most important variables are left undetermined to allow for a sensitivity optimization:

- Track reconstructed time: must be far from the beam flash to suppress in particular the RPC background.

- Track momentum: must be close to the one expected for CE: $\sim 105 \mathrm{MeV} / \mathrm{c}$. For lower momenta the signal is overwhelmed by DIO background while for higher ones cosmics dominate.

Finally, the complete set of cuts is presented in Tab. 3.1.

\subsection{Comparison with CD3 results}

As time goes on, it is natural that the experiment simulation changes both from the technical side (more realistic detector description) and from the software one. Changes have a direct impact on the expected background and new estimates are required every time these changes are considered relevant.

The last Mu2e "blessed" Sensitivity Update dates back to July 2017 ("CD3", [52]) where the expected schedule was not the "staged" one presented in Sec. 2.2, but a 


\begin{tabular}{|cc|}
\hline ID variable & cut value \\
\hline Reconstructed downgoing electron & $\mathrm{Q}=-1$ and $T_{0}<T_{\text {calo }}$ \\
Impact parameter & $-80 \mathrm{~mm}<d_{0}<105 \mathrm{~mm}$ \\
Tangent of the pitch angle & $0.5<\tan (\theta)<1$ \\
Helix maximum radial distance & $450 \mathrm{~mm}<d_{0}+2 R<680 \mathrm{~mm}$ \\
ANN for track quality selection & TrkQual $>0.8$ \\
Energy-momentum matching & $E / p>0.6$ \\
Time of flight & $-3.5 \mathrm{~ns}<T_{0}-T_{\text {calo }}<1 \mathrm{~ns}$ \\
CRV requirement & no CRV cluster in $\left[T_{0}-120 \mathrm{~ns}, T_{0}+50 \mathrm{~ns}\right]$ \\
\hline \hline Time in the center of the tracker & To be optimized \\
Momentum window & To be optimized \\
\hline
\end{tabular}

Table 3.1: Set of geometric, track-quality and PID cuts applied to identify the signal region in the current sensitivity update.

consecutive three-year run at full intensity (two batches mode). Since then, other relevant changes have occurred:

- The number of stopped muons / POT has decreased from 0.0019 to 0.0016. This is led by the modification of the Production Target geometry (Sec. 2.2.5).

- The CRV light yield assumed for CD3, was found to be overestimated. In addition, the CRV aging has been better quantified and resulted to be worse than originally expected.

- The achievable "extinction factor" is now estimated to be of the order of $10^{-12}$, well below the $10^{-10}$ requirement.

- Signal theoretical spectrum now includes losses due to the radiative corrections.

- From the track reconstruction point of view, the calorimeter-driven pattern recognition (CalPatRec) and the TrkCaloHit fit have been introduced.

- A better understanding of the systematics related to each background channel have been acquired.

All of these reasons has led to the 2020 Sensitivity Update project (shortly SU2020), to which the experiment is working since spring 2020.

While a full discussion of the expected sensitivity will be the subject of the last chapter, we want here to compare the background yields coming from the new simulations with the ones quoted at the CD3. Background events will be selected with the cuts listed in Tab. 3.1 and scaled to the CD3 expected data statistics of $3.6 \cdot 10^{20}$ POT $^{13}$. For the sake of comparison, the momentum and time windows will be assumed to be the same used at that time ${ }^{14}$ :

$$
\text { CD3 selections: } \quad p \in[103.85,104.9] \mathrm{MeV} / \mathrm{c} \quad t>700 \mathrm{~ns}
$$

Systematic uncertainties affecting each channel will be discussed here.

\footnotetext{
${ }^{13}$ Because of the dead time introduced by the Cosmic Ray Veto, the real total number of protons on target is a few percent higher.

${ }^{14}$ The momentum window was the result of an optimization with the 5-sigma discovery sensitivity as a figure of merit (see Chap. 5) while the time cut was set to make the in-time (exponential decreasing with time) and out-of-time (flat) RPCs equal.
} 
- CE signal: using $N_{\text {Gen }}=10^{6}$ generated CEs,including the radiative corrections, the global efficiency is estimated to be:

$$
\epsilon_{C E}=(10.44 \pm 0.03(\text { stat }) \pm 1(\text { sys })) \%
$$

This corresponds to the integral of the filtered red distribution in Fig. 3.2.

The statistical uncertainty comes from fluctuations in the number of accepted events per bin and was computed with

$$
\sigma_{i s t a t}=\sqrt{\sum_{i} \frac{\epsilon_{i}\left(1-\epsilon_{i}\right)}{N_{G e n}}}
$$

where $N_{i}$ is the bin occupancy and $\epsilon_{i}$ the bin efficiency $\left(N_{i} / N_{G e n}\right)$.

Systematic uncertainties arise from different effects: uncertainties in the magnetic field map or in the energy loss in the ST, gas leak, bunch intensity variations and accidental straw hits. Previous simulations of these effects gave a relative uncertainty of $11 \%$ on $\epsilon_{C E}([52])$.

- Cosmics: Fig. 3.16 shows the momentum distribution of the cosmic reconstructed tracks after the application of all the selection cuts but the CRV coincidence.
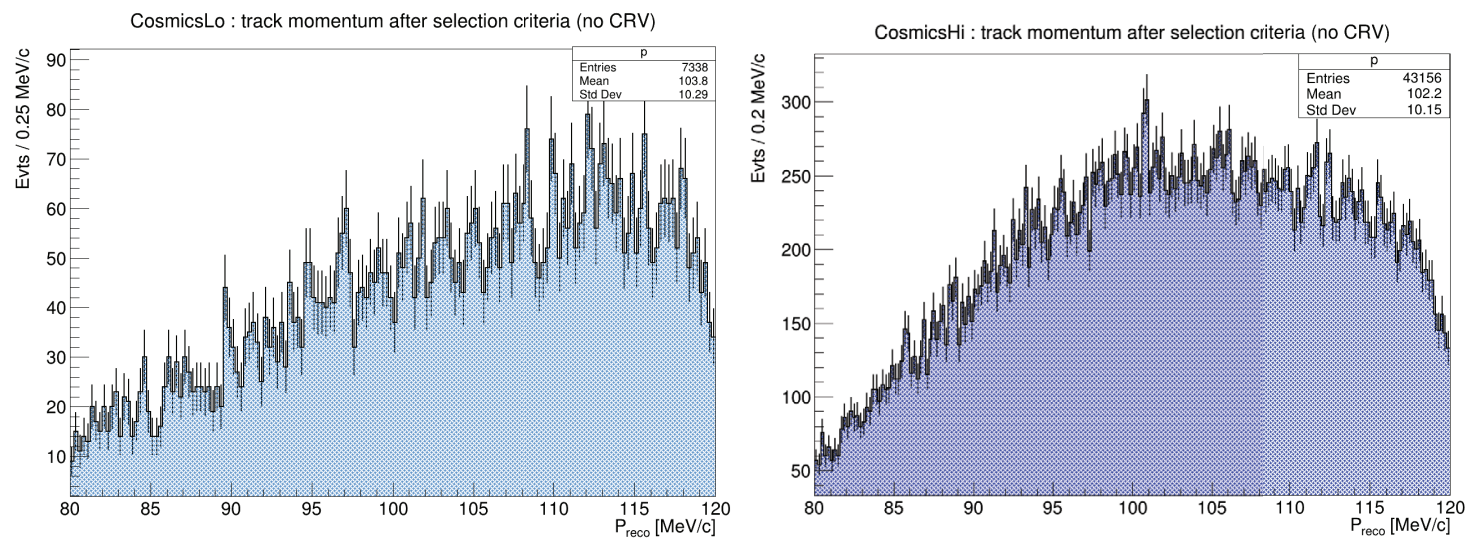

Figure 3.16: Cosmic tracks momentum distribution for the two datasets of the CRY3 simulation. All selection cuts have been applied except for the absence of the CRV cluster.

When the CRV cut is applied, only 237 events from the CosmicsLo sample and 15 events from the CosmicsHi sample are left in the entire momentum range. These are considered too few to get a reliable estimate of the reconstructed events within 103.85 $\mathrm{MeV} / \mathrm{c}<p<104.9 \mathrm{MeV} / \mathrm{c}$, in fact this window contains 9 events for CosmicsLo and none for CosmicsHi.

For this reason the integral between $103.85 \mathrm{MeV} / \mathrm{c}$ and $104.9 \mathrm{MeV} / \mathrm{c}$ has been performed using the distributions before the cut (Fig. 3.16) and the cut efficiency has been calculated in the entire momentum region $0<p<200 \mathrm{MeV} / \mathrm{c}^{15}$.

\footnotetext{
${ }^{15}$ This assumes that the coincidence between the CRV signal and the track does not depend on the momentum; this is a reasonable approximation in this momentum region.
} 
Based on the generated statistics of $3.83 \cdot 10^{8} \mathrm{~s}$ for CosmicsLo and $1.28 \cdot 10^{7} \mathrm{~s}$ for CosmicsHi, the total number of induced background from cosmic events is the sum of:

$$
\begin{gathered}
N_{C R l o}=0.16 \pm 0.02 \text { (stat.) } \pm 0.02 \text { (syst.) } \\
N_{C R h i}=0.31 \pm 0.08 \text { (stat.) } \pm 0.10 \text { (syst.) } \\
N_{C R}=N_{C R l o}+N_{C R h i}=0.47 \pm 0.08 \text { (stat.) } \pm 0.12 \text { (syst.) }
\end{gathered}
$$

Statistical uncertainties come from the squared sum of the Poissonian fluctuation of countings in the momentum interval and the uncertainty on the scaling factor with the same form as 3.15 (with 1 bin).

Systematic uncertainties derive from:

- The uncertainty on the total flux given by the usage of a specific generator. Recently, an alternative generator named CORSIKA [71] (with the simulation of hadronic particles at low energies handled by FLUKA [72]) has been used to evaluate the differences in charged and neutral particle fluxes at the generation and reconstruction level. The comparison has shown differences of the order of $\sim 10 \%$

- The uncertainty on the knowledge of the CRV efficiency. After becoming aware of the problem related to the aging of the CRV scintillators, the experiment has dedicated a great effort in the evaluation of the expected Light Yield (LY). The assumed number value of 28.5 PEs comes with its own systematic uncertainty of 0.7 PEs. Knowing that a $2 \mathrm{PE}$ loss would double the inefficiency, this can be translated into a $\sim 30 \%$ systematic on CosmicsHi background estimate.

- There is, in principle, a third component due to possible cracks or particularly inefficient detector of the CRV (hermeticity). However, it is not possible in the simulation to keep memory of the original particle crossing the CRV and the correspondent uncertainty has not been estimated. The problem has been partially mitigated increasing the overlap between the horizontal and vertical modules of the CRV.

The experiment is looking forward to operate a in situ measure of the cosmic background in absence of the beam: this will allow to reduce the systematic uncertainty due to this background.

- Decays in orbit: the integral of the weighted DIO distribution in the signal region [103.85, 104.9] MeV/c, after the application of all cuts (Fig. 3.17), gives:

$$
N_{D I O}=0.14 \pm 0.02(\text { stat }) \pm 0.11 \text { (syst) }
$$

In this last step, the strength of the momentum selection is greatly enhanced by the very low Czarnecki probability for a DIO in the signal region (the suppression is of the order of $10^{10}$ ).

Estimate of the statistical uncertainty on the DIO integral comes from the square root of the sum of the squares of the weights (Eq. 3.5) used to reproduce the theoretical spectrum. This formula can be shown to provide an unbiased estimate of the uncertainty (square root of the variance) of a weighted histogram integral when the weights are distributed normally [73]. The weight distribution of events in the signal 


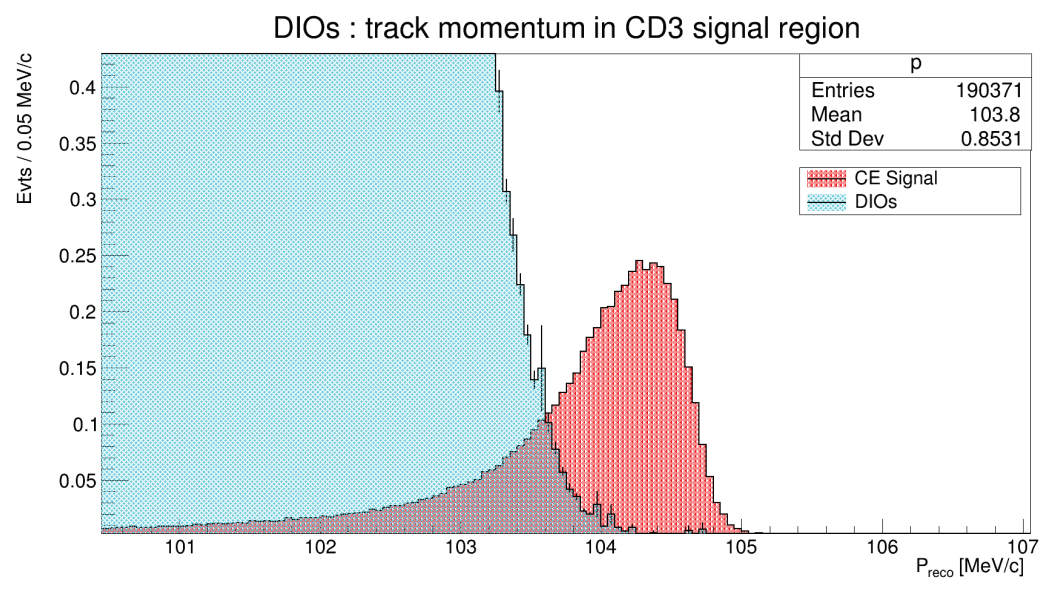

Figure 3.17: DIO reconstructed spectrum around the CE characteristic momentum. Only statistical uncertainties are shown. An indicative $R_{\mu e}=10^{-16}$ has been used for the signal histogram.

window is shown in [69] to be reasonably consistent with a log-normal distribution, and hence the estimate may be biased. In the current year, the experiment is working on a more robust estimate in case of log-normal distributed weights.

DIO related systematic uncertainties are:

- Theoretical uncertainties, concerning radiative corrections on higher-order terms neglected in the Czarnecki-Szafron formulation of Eq. 3.4 . One rough and conservative estimate is presented in the TDR of Mu2e [37] and considers the relative contribution of the radiative-corrected spectrum with respect to uncorrected one in the signal region: $\Delta N_{D I O}^{T h} / N_{D I O}=20 \%$;

- Tracker absolute momentum scale. That contribution was studied in the Mu2e TDR and might affect the results due to the fixed lower edge cut of the momentum signal window. TDR results are still valid: $\Delta N_{D I O}^{T r k S c a l e} / N_{D I O}=$ $45 \%$;

- Tracker momentum resolution. The result of a dedicated study is presented in [69]. For the estimate, a Toy MC was used to vary the number of background mixing events contained in the DIO sample from $\mathrm{x} 1$ to $\mathrm{x} 4$. The entity of the contamination could distort the DIO spectrum with mutual interactions between particles or degrade tracker efficiency. $\Delta N_{D I O}^{r e s} / N_{D I O}=54 \%$;

- Normalization factors used in the Eq. 3.7, i.e. intensity of the muon beam, the number of stopped particles, the number of out-of-target stopped muons, etc. This also is unchanged from the Mu2e TDR and is equal to: $\Delta N_{D I O}^{\text {norm }} / N_{D I O}=$ $9 \%$.

- Radiative pion captures: after selection cuts are applied to the two distributions in Fig. 3.8, the integral in the CD3 momentum signal region and $t>700$ ns gives:

$$
\begin{gathered}
N_{R P C e}=0.0029 \pm 0.0008(\text { stat }) \pm 0.0004(\text { syst }) \\
N_{R P C i}=0.0031 \pm 0.0008(\text { stat }) \pm 0.0005(\text { syst }) \\
N_{R P C}=0.0060 \pm 0.0011(\text { stat }) \pm 0.0009 \text { (syst) }
\end{gathered}
$$


Statistical uncertainties correspond to the square root of the sum of the squared weights for each event in the signal window.

Systematic uncertainties come from:

- The internal conversion side of the estimate is affected by the systematic uncertainty regarding its generation spectrum, which was assumed to be the same as the external one. Even assuming a flat photon spectrum instead of the Bistirlich's one, the number of RPC induced background events changes by less than $3 \%$.

- The values of the normalization parameters: $f_{R P C}$ and $\rho_{\gamma^{*} \rightarrow e^{+} e^{-}}$in Eq. 3.9 have been taken form the literature, and have their uncertainties. The former contributes with a $9.3 \%$ systematic uncertainty on both internal and external conversions (in-time and out-of-time), while the latter with a $5.5 \%$ only on the internal ones.

- A toy Monte Carlo was used to estimate the systematic uncertainty on the in-time contribution due to potential variations in the time shape of pulses of protons. A Gaussian timing shape was assumed as a baseline for testing and then variation on both the standard deviation and the specific shape used (uniform, hybrid) made possible to estimate the uncertainty due to the proton pulse timing shape as $10 \%$.

- It is important to note that the experiment is still working to estimate the systematic error due uncertainties on the pion production cross section. This could result to be dominant for RPC.

Main background estimates have been presented and are now collected in Tab. 3.2, where it is also possible to compare them with the CD3 results.

\begin{tabular}{c|c|c}
\hline Process & SU2020 estimates & CD3 estimates \\
\hline \hline Cosmics & $0.47 \pm 0.08$ (stat.) \pm 0.12 (syst.) & $0.209 \pm 0.022$ (stat.) \pm 0.055 (syst.) \\
\hline DIO & $0.14 \pm 0.02$ (stat.) \pm 0.11 (syst.) & $0.144 \pm 0.028$ (stat.) \pm 0.11 (syst.) \\
\hline RPC & $0.0060 \pm 0.0011$ (stat.) \pm 0.0009 (syst.) & $0.021 \pm 0.001$ (stat.) \pm 0.002 (syst.) \\
\hline Antiprotons & next chapter & next chapter \\
\hline
\end{tabular}

Table 3.2: Updated (LEFT) and previous (RIGHT) background estimates within the signal region $[103.85,104.9] \mathrm{MeV} / \mathrm{c}$ and $\mathrm{t}>700$ ns. Results are bench-marked for the past Mu2e schedule, i.e. a three-year, full intensity, run with $3.6 \cdot 10^{20}$ "live-time" POT.

The most significant change regards the cosmic background. The reason has been comprehended and lies in the significant lower (but more realistic) light yield assumed in the simulation for the CRV scintillators. The DIO estimate has not changed (it should be remembered that the shape is not an updated one). The RPC component is instead found to be lower than what was obtained at the CD3. This is because the previous result was assuming an extinction factor of $10^{-10}$ and in-time and out-of-time components were contributing at the same level. In the updated estimate a two order of magnitude lower extinction factor has been used and the out-of-time (internal and external) component has then been neglected. 
A dedicated discussion of the antiproton background will now be presented in the next chapter. 


\section{Chapter 4}

\section{The Antiproton Background}

Antiprotons are one of the most problematic sources of background for Mu2e. They are produced in the Production Target, by the same primary proton interactions responsible for the formation of pions, kaons and the subsequent muon beam. Having the same charge sign and a similar momentum to that of the wanted muons, antiprotons can be accepted by the Transport Solenoid, reach the Stopping Target and annihilate in other particles, especially pions, which themselves can decay producing photons, electrons or muons, all known sources contributing to the conversion electron background.

Given their large mass, antiprotons with momentum accepted by the TS, are significantly slower that pions or muons with similar momentum: a $\bar{p}$ of $100 \mathrm{MeV} / \mathrm{c}$ has a $\beta \simeq 0.1$, while pions with same momentum have $\beta \simeq 0.6$ and muons $\beta \simeq 0.7$. As a consequence, the $\bar{p}$ arrival time on the Stopping Target is much larger and the time window cut used to suppress the prompt background is not effective: this makes the antiproton background more dangerous.

In addition, the lack of experimental data for the antiproton production requires a theoretical model to fit the few existing cross section data and extrapolate it in the region of interest of Mu2e: this extrapolation gives a large contribution to the relative systematic error.

All of this requires to suppress the antiproton background as much as possible; this is the purpose of the set of absorbers and collimators placed in the TS described in Sec. 2.3.2. The material added to absorb the $\bar{p}$ has the side effect of increasing the pions created in their annihilations, this lately formed pions may reach the Stopping Target and produce an RPC background that is insensitive to the time rejection cut. This background will also be discussed in this chapter.

The estimate of $\bar{p}$ induced background is quite demanding from the computing point of view: in order to have $\mathrm{O}\left(10^{3}\right) \bar{p}$ s stopped in the Stopping Target it will be necessary to produce $\mathrm{O}\left(10^{15}\right)$ proton interactions in the Production Target! The generation time can be reduced using some expedients (phase space generation weights, event resampling at start of TS) that will be discussed in detail throughout the chapter.

\subsection{Antiproton production models}

Two reference frames have been used in the antiproton simulation:

- The $X s e c^{1}$ frame (Fig. 4.1), is a spherical coordinate system whose zenith axis

\footnotetext{
${ }^{1}$ Xsec" stands for "cross section".
} 
points along the incoming proton direction. This is the best frame to generate barps because of the azimuthal symmetry of the differential cross section.

- The Mu2e frame (Fig. 2.1) is the Cartesian coordinate frame, centered in the TS center, already defined in Sec. 2.1. It's used to propagate the particles through the detector. In this frame the $\bar{p}$ s emitted in the direction of the proton beam have a negative longitudinal momentum $p_{z}$.

Given the inclination of the proton beam with respect to the PS axis, the $\mathrm{z}$ axes of the two frames form an angle of $14^{\circ}$.

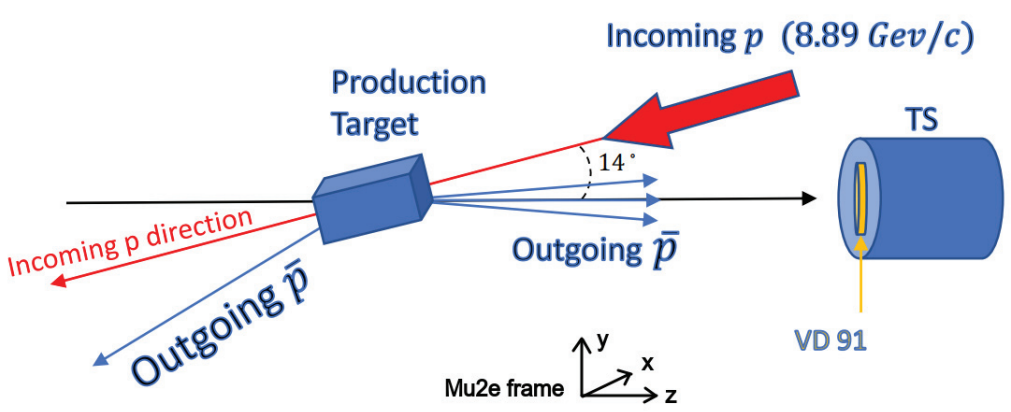

Figure 4.1: Scheme of the antiproton production. The incoming proton direction (red arrow) and the central axes of the PS (black arrow) lie on the z-x plane of the Mu2e frame. The origin of the Mu2e frame is in the central point of the TS, the region inside the PS is then z-negative.

The antiprotons are produced in the tungsten Production Target by the reaction:

$$
p+W \longrightarrow\left(W^{*}+\bar{p}\right)+X
$$

In the Xsec frame the production probability is flat in $\phi$, with a $\cos \theta$ and momentum (p) dependence given by the inclusive differential cross section. The basic underlying process is the well known reaction:

$$
p+p \longrightarrow(p+\bar{p})+p+p
$$

that, when close to the $\bar{p}$ production threshold ${ }^{2}$, cannot be described by QCD and requires the use of phenomenological effective theories. Theoretical predictions become even more difficult when the interactions of more nucleons is taken into account: multi-nucleon processes give a dominant contribution in some region of the production phase space and cannot be neglected.

\subsubsection{Kinematics of $p+p \longrightarrow(p+\bar{p})+p+p$}

Since Eq. 4.2 involves more than two particles in the final state, the closure of the kinematic is in general a complicated problem. An useful quantity turned out to be the maximum allowed energy for the antiproton in the center of mass system. This occurs when the system composed of the other 3 protons in the final state acts as a single body and recoils in the opposite direction with respect to the $\bar{p}$ (in the $\mathrm{CM}$ ).

\footnotetext{
${ }^{2}$ This is $T_{t h r}=5.63 \mathrm{GeV}$ (can be relaxed including the Fermi motion) that is very close to Mu2e proton beam energy: $T_{M u 2 e}=8.0 \mathrm{GeV}$.
} 
After have fixed the 4-momentum in the center of mass $\left(P^{*}\right)$ notation for the initial $(\mathrm{p} 1, \mathrm{p} 2)$ and final $(\bar{p}, 3 \mathrm{~N})$ state :

$$
P_{p 1}^{*}=\left(E_{p 1}^{*}, \vec{p}_{p 1}^{*}\right) \quad P_{p 2}^{*}=\left(E_{p 1}^{*},-\vec{p}_{p 1}^{*}\right) \quad P_{\bar{p}}^{*}=\left(E_{\text {max }}^{*}, \vec{p}_{\text {max }}^{*}\right) \quad P_{3 N}^{*}=\left(E_{3 N}^{*}, \vec{p}_{3 N}^{*}\right)
$$

and have taken the 4-momentum conservation into account:

$$
P_{T O T}^{*}=P_{p 1}^{*}+P_{p 2}^{*}=P_{\bar{p}}^{*}+P_{3 N}^{*}=(\sqrt{s}, \overrightarrow{0})
$$

one can write:

$$
\left\{\begin{array}{l}
P_{\bar{p}}^{*} \cdot P_{T O T}^{*}=E_{\max }^{*} \sqrt{s} \\
s=\left(P_{\bar{p}}^{*}+P_{3 N}^{*}\right)^{2}=m_{p}^{2}+\left(3 m_{p}\right)^{2}+2 P_{\bar{p}}^{*} \cdot P_{3 N}^{*}
\end{array}\right.
$$

The first equation can be inverted to obtain the desired quantity:

$$
E_{\text {max }}^{*}=\frac{P_{\bar{p}}^{*} \cdot P_{T O T}^{*}}{\sqrt{s}}=\frac{P_{\bar{p}}^{*} \cdot P_{\bar{p}}^{*}+P_{\bar{p}}^{*} \cdot P_{3 N}^{*}}{\sqrt{s}}
$$

and the second relation in the 4.3 can be used to express the result in terms on the Mandelstam $\mathrm{s}$ and the proton mass ${ }^{3}$ :

$$
E_{\text {max }}^{*}=\frac{s-\left(3 m_{p}\right)^{2}+m_{p}^{2}}{2 \sqrt{s}}
$$

from which also the correspondent $\bar{p}$ maximum momentum can be obtained:

$$
p_{\text {max }}^{*}=\sqrt{\left(E_{\text {max }}^{*}\right)^{2}-m_{p}^{2}}=\sqrt{\left(\frac{s-\left(3 m_{p}\right)^{2}+m_{p}}{2 \sqrt{s}}\right)^{2}-m_{p}^{2}}
$$

The kinematic described above can be generalized to the case of a general inclusive reaction $p+p \rightarrow \bar{p}+X$ with the substitution $\left(\left(3 m_{p}\right) \leftrightarrow M_{X}\right)$, where $M_{X}$ is the mass of the final state particle system except for the antiproton. This is useful to understand the specif parameterization that will be presented in Sec. 4.1.3 as a function of

$$
x_{R} \equiv \frac{E^{*}}{E_{\max }^{*}}
$$

and the transverse momentum of the antiproton $p_{T}$ in the laboratory system.

Generalizing, all the introduced variables in the center-of-mass have their correspondent in the laboratory system. The boost will be always assumed to be the one corresponding to a proton collision on a single nucleon target at rest (the incoming proton direction is aligned with the z-axis of the Xsec frame) with the velocity of the center-of-mass defined by:

$$
\beta_{C M}=\left(0,0, \frac{p_{p 1}}{E_{p 1}+m_{p}}\right)
$$

\subsubsection{Available measurements for antiproton production}

While plenty of data and reliable parameterizations exist for the high-energy regime $\left(p_{p}>100 \mathrm{GeV} / \mathrm{c}\right)$, in the region of interest for Mu2e, that is $E_{\text {proton }} \sim 9 \mathrm{GeV}$ and backward production, very few data, summarized in Table 4.1 , are available.

\footnotetext{
${ }^{3}$ It has been assumed that the mass of the proton is equal to the mass of the nucleon
} 


\begin{tabular}{ccccc}
$N_{\text {points }}$ & $P_{p}(\mathrm{GeV})$ & $\theta_{\bar{p}}\left(\mathrm{Xsec}^{\circ}\right)$ & $P_{\bar{p}}(\mathrm{GeV})$ & Origin \\
\hline 2 & 10 & 0 & $1.06,1.40$ & Tungsten, Amann et al [74] \\
13 & 10 & 3.5 & $1.25 \div 4.50$ & Tantalum, Sibirtsev et al [75] \\
5 & 10 & 10.5 & $0.73 \div 2.47$ & Tantalum, Kiselev et al [76] \\
8 & 10 & 10.8 & $0.72 \div 1.87$ & Gold, Barabash et al [77] \\
8 & 10 & 59 & $0.58 \div 1.35$ & Tantalum, Kiselev et al [76] \\
4 & 10 & 97 & $0.60 \div 1.05$ & Tantalum, Boyarinov et al [78] \\
2 & 10 & 119 & $0.59,0.66$ & Tantalum, Boyarinov et al [78]
\end{tabular}

Table 4.1: Available data for antiproton production from proton interactions on different heavy nuclei.

\subsubsection{Striganov's model}

Within Mu2e, the first attempt to reproduce the experimental data and to extrapolate them in the whole phase space has been done in 2011 by Sergei Striganov [79]. This model starts from the parameterization of the Lorentz-invariant differential cross section for the inclusive hadron production $p+p \rightarrow h+X$, made by L. C. Tan and L. K. Ng (1983, $[80])$ :

$$
\left(E \frac{d^{3} \sigma}{d p^{3}}\right)_{R S}\left(x_{R}, p_{T}\right)=f\left(x_{R}\right) e^{-\left(A\left(x_{R}\right) \cdot p_{T}+B\left(x_{R}\right) \cdot p_{T}^{2}\right)} \quad\left(m b \cdot G e V^{-2} \cdot c^{3}\right)
$$

with:

$f=a_{1} e^{\left(-a_{2} x_{R}\right) \theta\left(a_{3}-x_{R}\right)}+\left(\sigma_{00}-a_{1}\right)\left(1-x_{R}\right)^{a_{4}}$

$A=a_{5} e^{-a_{6} x_{R}}+a_{7} e^{a_{8} x_{R}}$

$B=a_{9} e^{\left[-a_{10}\left(x_{R}+a_{11}\right)\right]}\left(x_{R}+a_{11}\right)^{a_{12}}$

$\theta(u)= \begin{cases}0 & \text { for } u<0 \\ 1 & \text { for } u \geq 0\end{cases}$

(The $a_{i}$ parameters are fixed to describe the specific reaction $p+p \rightarrow \bar{p}+X$ ). This parameterization works well and is considered reliable in the high energy region, $\sqrt{s}>10$ $\mathrm{GeV}$ where plenty of data for antiproton production exists and the cross-section is also independent on $\sqrt{s}^{4}$.

What is instead needed for Mu2e is the low energy regime. The authors themselves propose a procedure to extrapolate results to the low-energy (LE) $(\sqrt{s}<10 \mathrm{GeV})$ region, with the usage of a scaling ratio $(\mathrm{R})$ parameterized in terms of $\sqrt{s}$ and a new "scaling variable" $x_{T}$ :

$$
\begin{gathered}
x_{T} \equiv \frac{x_{R}-x_{m}}{1-x_{m}} \quad \text { where } x_{m}=m c^{2} / E_{\text {max }}^{*} \text { is the minimum value of } x_{R} \\
R \equiv \frac{\left(E d^{3} \sigma / d p^{3}\right)_{L E}}{\left(E d^{3} \sigma / d p^{3}\right)_{R S}}\left(\sqrt{s}, x_{T}\right)^{5}=\left(1-\exp \left[-\left(1-\left(\exp \left(-A\left(x_{T}\right) Q^{B\left(x_{T}\right)}\right)\right) \exp \left(C\left(x_{T}\right) Q-D\left(x_{T}\right)\right)\right)\right]\right)^{-1}
\end{gathered}
$$

\footnotetext{
${ }^{4}$ The so called radial-scaling (RS) ( Taylor et al 1976, [81]) represents the reason why Eq. 4.9 has no $\sqrt{s}$ dependence.

${ }^{5}$ the $p_{T}$ dependence has been observed to be very soft and thus dropped in the presented parameterization.
} 
with:

$A=b_{1} e^{-b_{2} x_{T}}$

$B=b_{3} e^{b_{4} x_{T}}$

$C=b_{5}+b_{6} x_{T}+b_{7} x_{T}^{2}$

$D=b_{8} e^{b_{9} x_{T}}$

$Q=\sqrt{s}-\sqrt{s_{t}}$ (being $\sqrt{s_{t}}$ the value at the $\bar{p}$ production threshold)

The procedure is supported only for the authors available data region, which includes only forward production. S. Striganov took this analytical form and extrapolated down to the Mu2e energy. In the absence of further data, he also assumed the model to be extendable to all the phase space, including backward production.

Then, in order to extend the presented $p+p$ cross section parameterization to heavy targets, Striganov made use of a multiplicative factor equivalent to the ratio between the total inelastic cross section for proton on heavy targets (with an atomic mass A) and the ones for a $\mathrm{p}+\mathrm{p}$ collision:

$$
F_{h . N .}(A)=\frac{\sigma_{p+A}^{\text {tot.inelastic }}(A)}{\sigma_{p+p}^{\text {tot. inelastic }}(A=1)}
$$

The total cross section is given, for a generic atomic weight "A", by the following formula [82] (for $E_{p}>2 \mathrm{GeV}$ ):

$$
\sigma_{p+A}^{\text {tot.inelastic }}(A)=45 \cdot A^{0.7} \cdot(1+0.016 \sin (5.3-2.63 \ln (A)))
$$

Then, varying the atomic weight, the model was adapted to different target nuclei. To give an indicative value, this ratio is $\sim 1710(\mathrm{mb}) / 45(\mathrm{mb})=38$ for Tungsten $(\mathrm{A}=183.84)$.

Fig. 4.2 shows the Striganov's parameterization of the antiproton production Lorentzinvariant cross section obtained from the described combination (for $\sqrt{s}<10 \mathrm{GeV}$ ):

$$
\left(E \frac{d^{3} \sigma}{d p^{3}}\right)_{L E}\left(\sqrt{s}, x_{R}, p_{t}, A\right)=\left(E \frac{d^{3} \sigma}{d p^{3}}\right)_{R S}\left(x_{T}\left(x_{R}\right), p_{t}\right) \cdot R\left(\sqrt{s}, x_{R}\right) \cdot F_{h . N}(A)
$$

superposed to the data ${ }^{6}$.

\subsubsection{An alternative model}

Striganov's model has various known weaknesses:

- The proposed parameterization is complicated and just empirical: there's no try to justify the adopted functional forms with a physics model.

- This model assumes the extrapolation from Tan and Ng to be valid also for backward production, even if it is supported only in the forward one (and same parameters $a_{i}$ and $b_{i}$ are used for both regions). It is hard to believe the physics for a backward produced antiproton to be the same as a forward production. In particular, it is not even possible to create a $\bar{p}$ in the backward direction in a $\mathrm{p}\left(E_{1}=8.9 \mathrm{GeV}\right)+\mathrm{p}$ collision, for which multi-nucleon processes need to be taken into account.

\footnotetext{
${ }^{6}$ we imply the change of variables from $x_{R}, p_{t}$ to the momentum and angle in the laboratory frame
} 


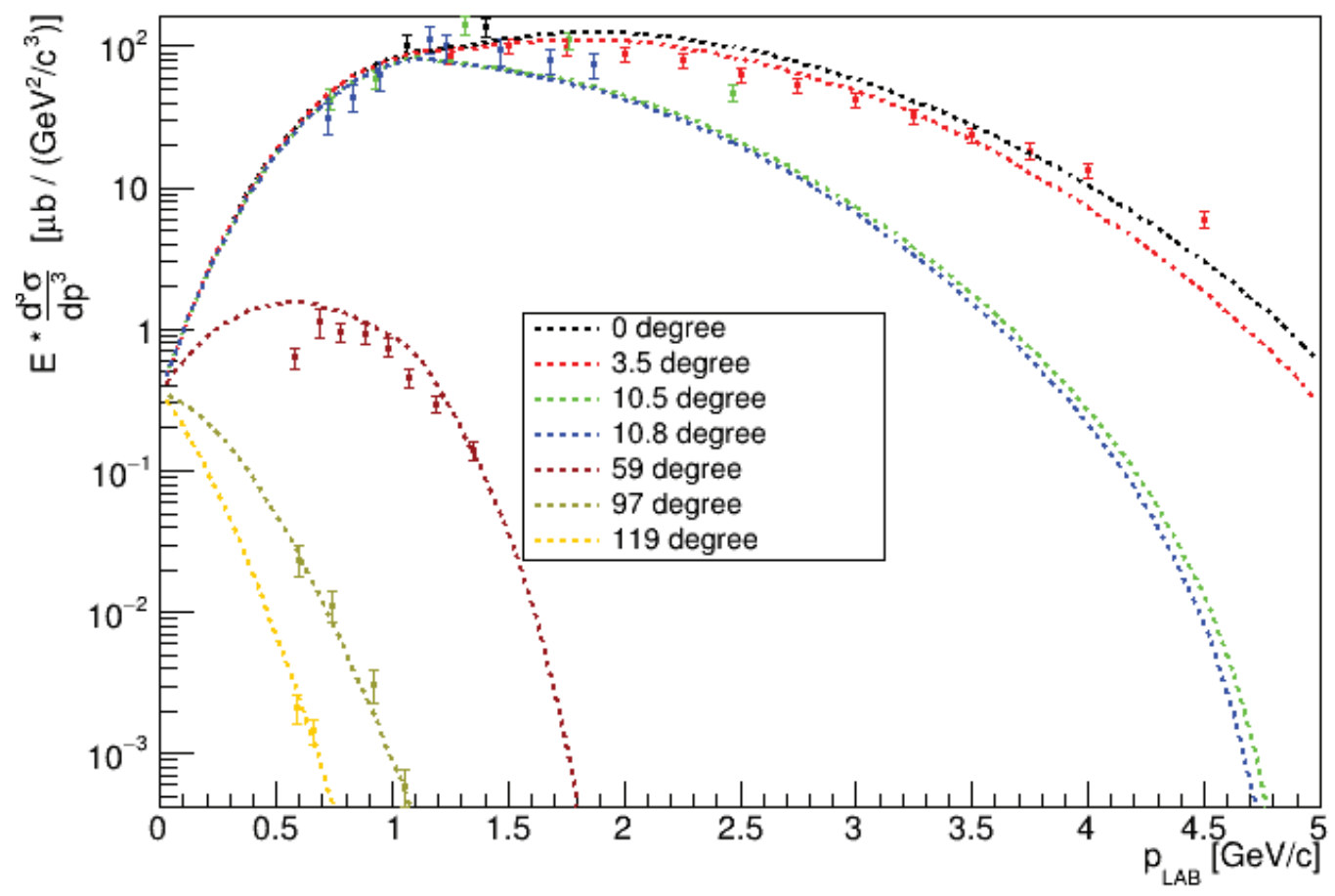

Figure 4.2: S. Striganov model curves superposed to the data points described in Table 4.1. The $\mathrm{y}$ axis shows the Lorentz invariant cross-section while $\mathrm{x}$ axis shows the magnitude of the outgoing antiproton momentum in laboratory frame.

- Even if extrapolation might be assumed to be reliable, push it close to the $\bar{p}$ production threshold requires attention. However, no threshold effects are included in this model.

- L. C. Tan and L. K. Ng differential cross section has been built to describe protonproton collisions. It's clear that this cannot remain unchanged for the collision with heavy targets. Saying that total inelastic cross-section for protons and differential for $\bar{p}$ production scale in the same way, is equivalent to assuming the relative fraction of produced antiprotons to be te same no matter the target.

- The procedure does not involve a fit to the data, only showing data superposed to the parameterization. As a consequence the uncertainty matrix of the fit is not available and it is thus not possible to accurately estimate the systematic uncertainty on the cross section.

- The parameterization seems not to describe the data accurately in the forward production region, at relatively high momentum (red, green and blue points), which was actually supposed to be the most reliable one.

All these considerations have brought to the birth of an alternative model, developed by R.H. Bernstein ( 2019, [83]) with my help.

The new model starts calculating the invariant cross section, $E \frac{d^{3} \sigma}{d p^{3}}$ in the center-ofmass system $(\mathrm{CM})$. In this reference frame the calculation is much simpler, since there 
is no dependence on the polar angle $\theta_{c m}$ : there is indeed no privileged direction for the antiproton production in the process of aggregations of sea quarks contained in the nucleons. The cross section dependence on the $\bar{p}$ momentum in the laboratory frame, that is needed to fit the experimental data, is then obtained using a standard Lorentz variable transformation.

To extend the formula 4.6 obtained for $p+p \longrightarrow(p+p+p+\bar{p})$ to the case of interaction with more nucleons, we can just replace the mass of the target proton with one of $\mathrm{N}$ nucleons:

$$
s_{p p}=2 m_{p}^{2}+2 E_{p 1} m_{p} \longleftrightarrow s_{p N}=m_{p}^{2}+\left(N m_{p}\right)^{2}+2 E_{p 1}\left(N m_{p}\right)
$$

and obtain the most general case $p+N \longrightarrow \bar{p}+X$ :

$$
p_{\text {max } N}^{*}=\sqrt{\left(\frac{s_{p N}-\left(M_{X}\right)^{2}+m_{p}}{2 \sqrt{s_{p N}}}\right)^{2}-m_{p}^{2}}
$$

The maximum antiproton momentum in the laboratory frame can be higher when more nucleons are considered as target.

We then define the variable:

$$
x_{C M} \equiv \begin{cases}\frac{p^{*}}{p_{\text {max }}^{*}} & \text { if } \cos \left(\theta_{c m}\right) \geq 0 \\ -\frac{p^{*}}{p_{\text {max }}^{*}} & \text { if } \cos \left(\theta_{c m}\right)<0\end{cases}
$$

that is the ratio between the antiproton momentum $\left(p^{*}\right)$ and the maximum antiproton momentum allowed in case of $p+p$ collision $\left(p_{\max }^{*}\right)$, both in the CM. The ratio receives a negative sign in case of backward production (in the CM) to allow to separately treat the forward and backward production with respect to the incident proton. It must be noted that in case of interaction with more nucleons (a 'multi-nucleon state'), the ratio can be larger than one, in absolute value.

The minimum number of nucleons needed to have a given value of $x_{C M}$ is called cumulative number and denoted with the greek letter $\eta$. It is obtained by finding the minimum value of $\mathrm{N}$ for which $p^{*}<p_{\max N}^{*}$, for this reason it may be not integer. Fig. 4.3 shows the cumulative number as function of the ratio $x_{C M}$ : it can be observed that high-momentum backward events $\left(x_{C M}<-1\right)$ need to be produced by a multinucleon state $(\eta>1)$. The behavior below $x_{C M}=-1$ can be interpreted as a linear dependence with a proportionality constant with module lower than 1, so that less than 2 nucleons are required to reach $x_{C M}=-2$.

Fig. 4.5 shows the invariant cross section according to this presented model against $x_{C M}$ :

- For $x_{C M}<-1$ the cross section is well fitted by a simple exponential. This is in agreement with what has been observed at JINR (Dubna) and ITEP (Moscow) and deeply studied by S. S. Shimanskiy (2014, [84]), Yu. T. Kiselev et al (2012, [76]) and G.A. Leksin [85].

In the analytical formula, the exponential piece will be expressed in terms of the $\bar{p}$ kinetic energy (instead of momentum) to be consistent with the mentioned papers. 


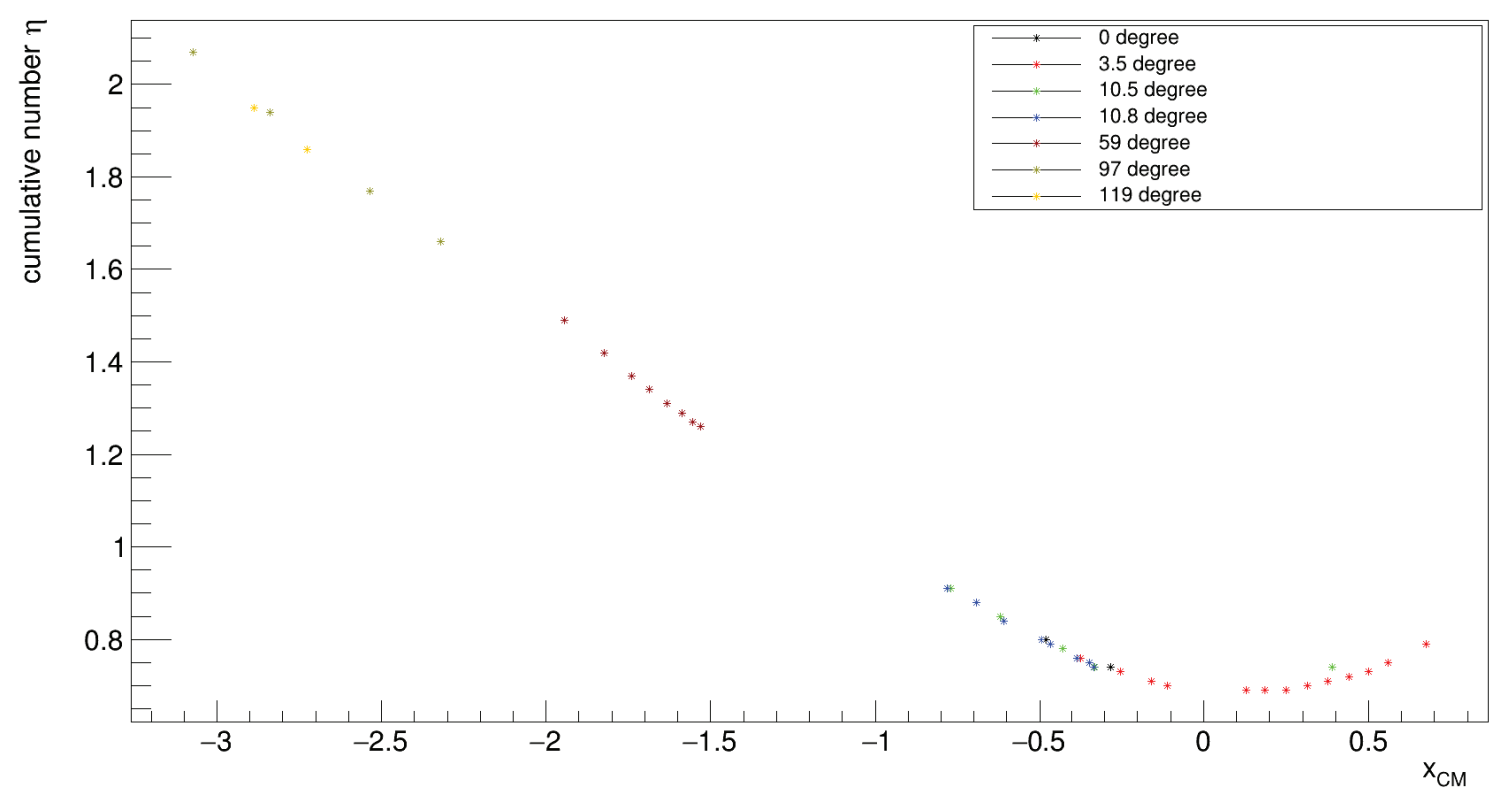

Figure 4.3: Cumulative number $(\eta)$ as a function of $x_{C M}$ for all the data available for $\bar{p}$ production: various data correspond to $\eta>1$ but only one to $\eta>2$.

An additional factor $S(\theta)$ can be introduced to take into account the probability of a $\bar{p}$ to be absorbed when it crosses the nucleus. Considering as example the $\bar{p}$ s produced in the very first layer of the Tungsten nucleus, the fraction of antiprotons that cross the nucleus without interacting is $e^{-\frac{L\left(\cos \left(\theta_{c m}\right)\right)}{\lambda_{I}}}$, where $\mathrm{L}$ is the geometrical path length inside the nucleus, that depends on $\theta_{c m}$ (Fig. 4.4).

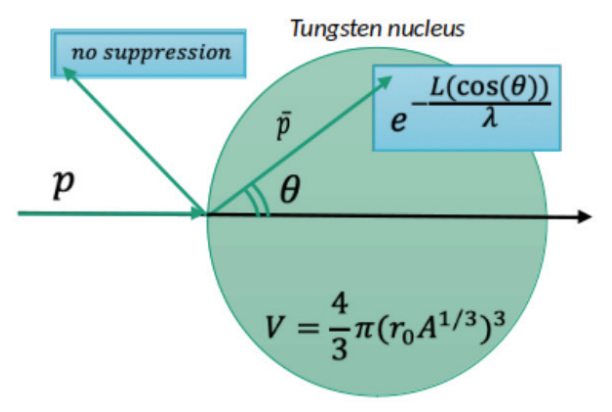

Figure 4.4: Graphical scheme of the forward suppression. We supposed the interaction to take place in the very first layer of the Tungsten Nucleus

The nuclear interaction length $\lambda_{I}$ scales with the nuclear density $\left(\rho_{\text {nuclear }}=2.3\right.$. $10^{14} \mathrm{~g} / \mathrm{cm}^{3}$ ) but in case of interaction with a multi-nucleon, that is for $\eta>1$, the effective density to be considered is much larger: according to the paper by G.A Leksin [85] the density of a multinucleon state is a factor $2^{3}$ bigger than the typical one inside a nucleus. In case of interaction with tungsten nucleus, the interaction length for $\eta>1$ becomes significantly shorter than the nucleus radius $\left(r_{W}=6.82 \mathrm{fm}\right)$ :

$$
\rho_{W} \lambda_{I}=191.9 \mathrm{~g} / \mathrm{cm}^{2}(\text { from PDG }) \longrightarrow \frac{\rho_{W}}{\rho_{\text {nuclear }}} \lambda_{I}= \begin{cases}8.3 \mathrm{fm} \sim r_{W} & \text { if } \eta \leq 1 \\ 1.04 \mathrm{fm}<<r_{W} & \text { if } \eta>1\end{cases}
$$


Using a drastic approximation ${ }^{7}$, in case of interaction with a multinucleon $(\eta>1)$, the model introduces a suppression factor $S\left(\theta_{\mathrm{cm}}\right)$ that completely suppresses the forward production, leaving the backward production unaffected:

$$
S\left(\theta_{c m}\right)=\left\{\begin{array}{ll}
0 & \text { if } \cos \left(\theta_{c m}\right)>0 \text { (forward production) } \\
1 & \text { if } \cos \left(\theta_{c m}\right) \leq 0 \text { (backward production) }
\end{array} \text { for } \eta>1\right.
$$

- For $\left|x_{C M}\right|<1$ the cross section is fitted by a simple Gaussian. This is just an empirical finding with no particular physical explanation.

At the moment there is no implementation of the $S\left(\theta_{c m}\right)$ effect in case of $\eta<1$. The path inside the Tungsten and the interaction length are comparable and the cross section dependence on $\theta_{C M}$ becomes not trivial. This is most probably the origin of the $\theta_{C M}$ asymmetry observed in the data in Fig. 4.5. To take into account this asymmetry an additional parameter, corresponding to the Gaussian mean $\left(O_{G}\right)$ has been included in the fit function.

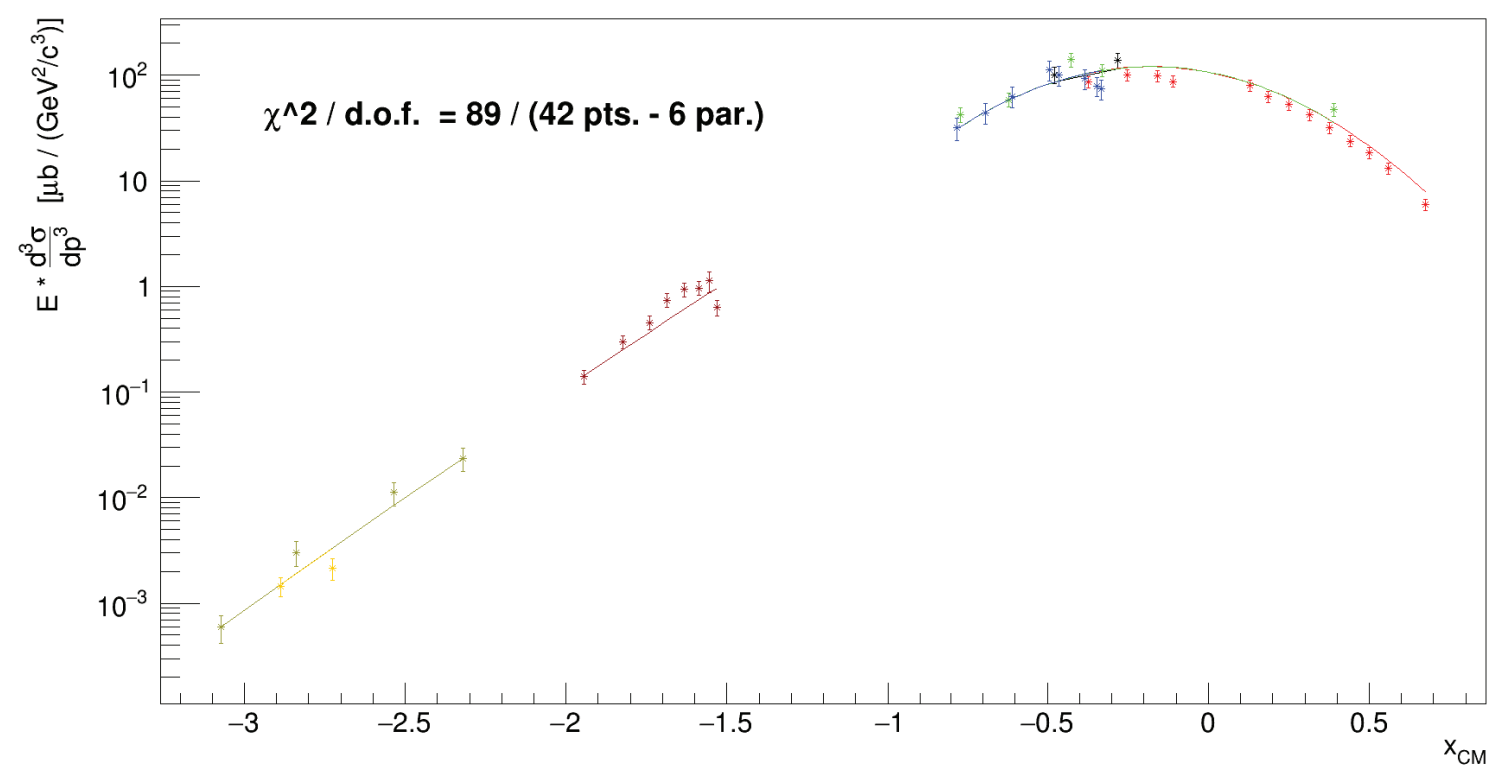

Figure 4.5: Invariant cross section as a function of $x_{C M}$ for all data points. The fit with the alternative model by R. Bernstein is showed.

Finally the model parameterization of the antiproton production cross section is now shown:

$$
E \frac{d^{3} \sigma}{d p^{3}}= \begin{cases}N_{G} \frac{1}{\sqrt{2 \pi \sigma_{G}^{2}}} e^{-\frac{\left|x_{c m}+O_{G}\right|^{2}}{2 \sigma_{G}^{2}}} & \text { for }\left|x_{C M}\right| \leq 1 \\ N_{E} S_{E}(\theta) e^{-\left|\frac{K^{*}\left(x_{c m}\right)}{E_{\text {max }}^{*} O_{E}}\right|} & \text { for }\left|x_{C M}\right|>1\end{cases}
$$

With free parameters being:

- $N_{G}$ : Normalization for the gaussian term

\footnotetext{
${ }^{7}$ This approximation could be improved if more $\bar{p}$ data will be available.
} 
- $\sigma_{G}$ : Sigma for the gaussian

- $O_{G}$ : Shift that determines the mean of the gaussian

- $N_{E}$ : Normalization for the exponential term

- $O_{E}$ : Shape of the exponential in unity of $E_{\max }^{*}$

The above function has been used to fit the data using the minimum $\chi^{2}$ technique. Unfortunately most of the papers reporting the $\bar{p}$ production probability do not quote the systematic error associated to the normalization. Since the normalization error is common to all the data taken at the same angle, to account for the lack of systematic uncertainties on the normalization one parameter per angle dataset has been added to the fit. The complete expression of the $\chi^{2}$ to be minimized is then:

$$
\chi^{2}=\left(\frac{y_{i}-\left(1+N_{k}\right) f\left(x_{i}\right)}{\sigma_{i}}\right)^{2}+\left(\frac{N_{k}}{\sigma_{N_{k}}}\right)^{2}
$$

with $y_{i}$ being the data points, $f\left(x_{i}\right)$ the corresponding model prediction, $N_{k}$ the normalization parameter corresponding to the k-th angle data set, $\sigma_{i}$ the uncertainty on the i-th point and $\sigma_{N_{k}}$ the uncertainty on $N_{k}$ (assumed to be $10 \%$ for all angle datasets). Fig. 4.5 shows the result of the fit: $\chi^{2} /$ d.o.f. $=89 /(42$ pts. -6 par.).

One of the most important result is that the covariance matrix from the fit is now accessible. The uncertainties on the fit parameters can then been used to obtain the uncertainty on the invariant cross section in different points of the phase space: relative errors are $\sim 10 \%$.

\subsection{Antiproton simulation}

The generation of the antiprotons in the production target and their tracing from the PS to the detectors is done using the Mu2e Offline software. The simulation of particle interactions is based on Geant4 (G4) [86] while the event processing is governed by the art framework [87] and the data handling by the $S A M$ system, these last both developed at Fermilab.

To optimize the time needed for the generation and the use of computing resources the simulation has been divided in 7 stages, from S0 to S6: There are 7 different stages in the simulation (Fig. 4.6) that will be briefly touched here and extensively discussed in the later sections:

- Stage 0: Primary beam protons are generated and propagated inside the Production Target. Whenever an inelastic interaction (producing antiprotons or not) occurs in the target, the vertex position is stored and the propagation stopped.

- Stage 1 : One antiproton is generated from each S0 interaction vertex according to a certain momentum (p) and angle $(\cos \theta)$ probability density function. Events are then propagated inside the Production Target and the Production Solenoid. Only antiprotons that reach a virtual detector (VD) in front of the TS entrance (before the COL1 $\bar{p}$ absorber window ) are saved and used as the input for the next stage. 


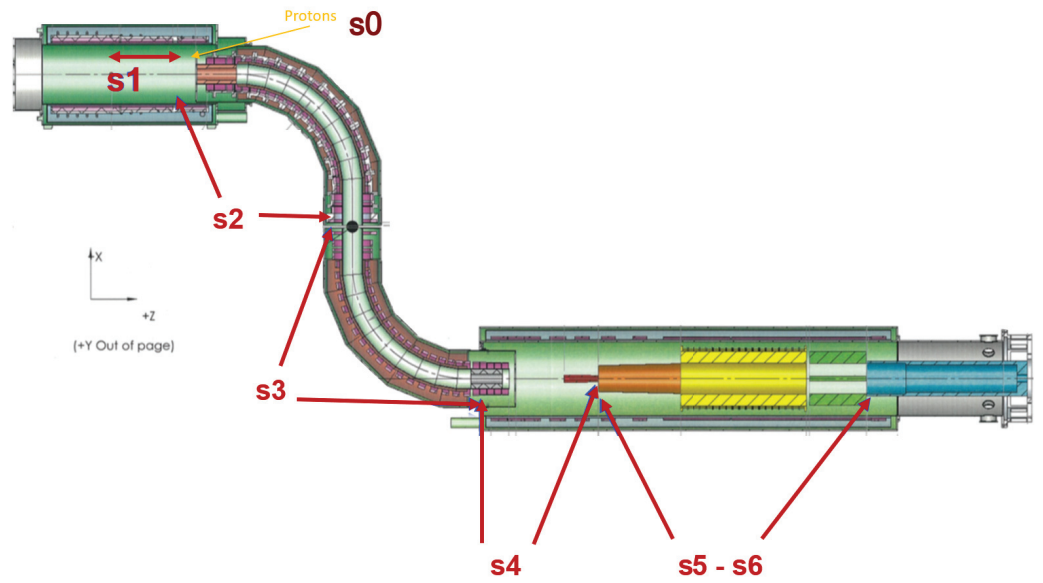

Figure 4.6: Scheme of the different stages of the antiproton background simulation.

- Stage 2: Antiprotons are here propagated from TS entrance to the exit of COL3u, right in front of the titanium window in the middle of the TS. From this stage, $\pi^{-}$ produced from spurious interactions are also stored and traced. In order to increase the available statistics, a re-sample factor is applied at this stage: every $\bar{p}$ from $\mathrm{S} 1$ is traced multiple times, producing independent events thanks to the statistical fluctuations of the energy losses in the absorbers. A resampling factor of $10^{5}$ still keeps the oversampling ${ }^{8}$ under control.

- Stage 3: Transportation of $\bar{p}$ and $\pi^{-}$from COL3u exit to the TS5 entrance.

- Stage 4: Transportation of $\bar{p}$ and $\pi^{-}$from TS5 to the Stopping Target. Here, positions of the stopped antiprotons and pions are stored.

- Stage 5: Re-sample of S4 vertices: $3 \cdot 10^{7}$ interacting stationary antiprotons and pions are created at rest using the time and the position of the stops in the stopping target. Particles produced in the $\bar{p}$ annihilation at rest or from the photon radiated during the pion capture in the nucleus are then propagated to the detectors.

- Stage 6: Finally, the reconstruction from hits in the detectors is performed and CE-like track are selected.

The number of fake CE tracks can be obtained from:

$$
\bar{p} \text { induced background }=N_{P O T} \times \mathcal{P}(\bar{p} \text { stop. } \mid P O T) \times \mathcal{P}\left(e^{-} \text {reco. } \mid \bar{p} \text { stop. }\right)
$$

where $N_{P O T}$ is the number of protons on target. The first factor is estimated with the output of the chain $\mathrm{S} 0 \div \mathrm{S} 4$, while $\mathrm{S} 5$ and $\mathrm{S} 6$ are dedicated to the estimate of the second one.

For what concerns the CPU time, Stage 0 and 1 are the most time expensive, Stage 2 simulation can be at the same level in case of an high resampling factor, while the following stages are an order of magnitude faster.

\footnotetext{
${ }^{8}$ The fact that a significant fraction of events comes from the same original antiproton.
} 


\subsection{S0: Proton inelastic interactions}

In S0 $10^{10}$ protons are generated at the Mu2e proton-beam kinetic energy of $8 \mathrm{GeV}$ and then propagated through the Haymann2 Production Target. Every proton is traced independently. If an inelastic interaction occurs during its propagation inside the PT, the position is saved, even if no antiprotons are in principle produced. If it undergoes multiple interactions, only the first one is saved. If instead there is no interaction, the propagation of the proton is continued outside the target until it naturally stops inside the internal surface of the PS.

The total probability of an inelastic interaction depends on the proton $\sigma_{\text {tot. inelastic }}$ on tungsten, for which an empirical formula for high-energy ( $\mathrm{E}>2 \mathrm{GeV}$ ) was given in 4.13. For $\mathrm{A}=183.84$, it gives:

$$
\sigma_{\text {tot.inelastic }}(W)=(1710 \pm 30) \mathrm{mb}
$$

The fraction of interacting protons is calculated as:

$$
f_{0} \equiv \frac{\text { interacting protons }\left(n_{\text {int }} \geq 1\right)}{P O T}=0.792
$$

For those who interact, the first vertices distribution in the $\mathrm{z}$ Mu2e coordinate is shown in Fig. 4.7. Protons interactions in the simulation are handled by G4.

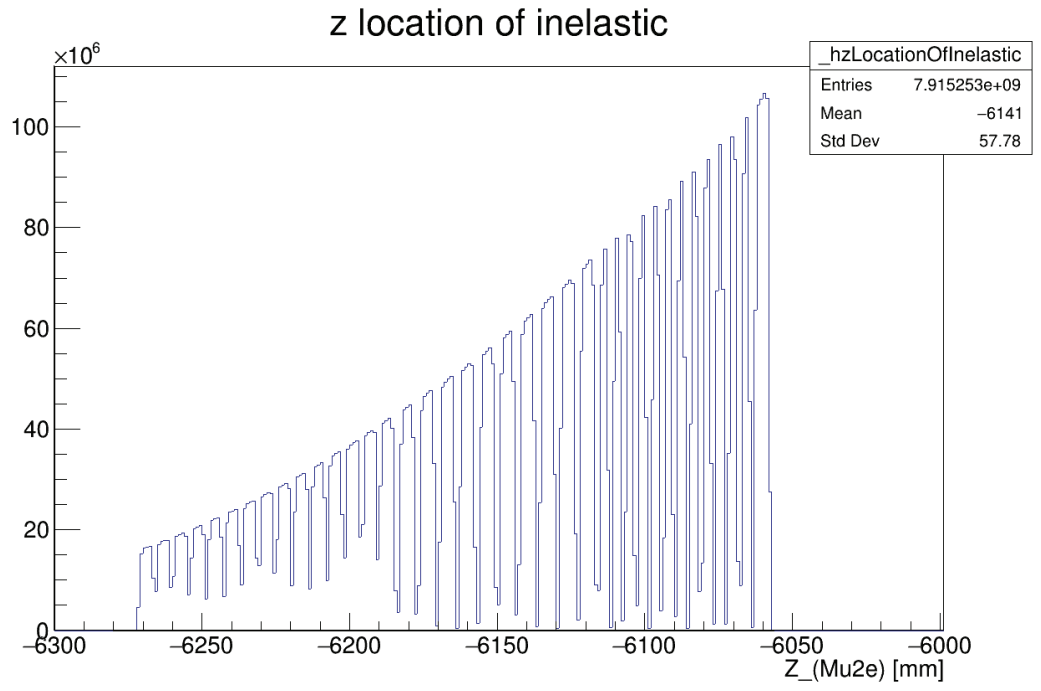

Figure 4.7: Z-coordinate, in the Mu2e frame, of the first proton inelastic interactions inside the Production Target. The segmentation of the PS is visible.

Since the only first vertex is saved, the probability for a proton to survive the interaction and produce a secondary proton, which can interacts itself (and possibly produce an antiproton), is being neglected. Then, it is in principle possible that the estimate will be biased. However, given the thinness of the PT $(<1 \mathrm{~cm})$ and the interaction length for protons in Tungsten $\left(\lambda_{I}=10 \mathrm{~cm}\right)$, the majority of the interaction products are directed toward the outside of the target and the probability to have a second hadronic inelastic interaction inside it can be neglected. 


\subsection{S1: Antiprotons generation to TS entrance}

During stage 1, one antiproton is generated from each S0 vertex and traced inside the Production Solenoid. This procedure allows to replace the G4 model for antiproton production with the ones described in Sec. 4.4.1. In addition this helps increasing the statistics but, since not all the proton inelastic interactions produce antiprotons, the number of generated $\bar{p}$ using $\mathrm{S} 0$ interaction vertices has to be re-scaled by the ratio:

$$
\frac{\sigma_{p A \rightarrow \bar{p} X}}{\sigma_{\text {tot. inelastic }}}
$$

where $\sigma_{p A \rightarrow \bar{p} X}$ is the total inclusive cross section for $\bar{p}$ generation from proton interactions on a nucleus of mass A. The $\bar{p}$ production cross section can be obtained by integrating the differential cross section over the full $\left(x_{C M}(p), \cos (\theta)\right)$ phase space. The result significantly depends on the model:

$$
\sigma_{p A \rightarrow \bar{p} X}= \begin{cases}97.86 \mu b & (\text { Striganov's model }) \\ 30.03 \mu b & (\text { Bernstein's model })\end{cases}
$$

\subsubsection{Antiproton Generators}

Given the uncertainty on the model to be used for $\bar{p}$ generation, it's convenient to generate the antiprotons using a simple model and then apply the weights needed to reproduce the wanted phase space distribution. In this way a unique generation can be used to study the results of more models. Two different options have been studied:

- A flat generator producing uniform distributions for the $\bar{p}$ momentum $p$ and $\cos (\theta)$ : this is the simplest generator and the distribution of $p$ and $\cos (\theta)$ at the end of each stage directly provide the stage acceptances as function of these variables.

- A weighted generator may be used to enhance the statistics in the regions that are more critical for the $\mathrm{CE}$ background so reducing the statistical uncertainty on the background being equal the amount of computing resources used. To obtain the acceptance as function of the $p$ and $\cos \theta$ the distributions of these variables at the end of each stage need to be "unweighted" according to the unweighting factor:

$$
U(p, \cos \theta)=\frac{P_{\text {flat }}(p, \cos \theta)}{P_{\text {weighted }}(p, \cos \theta)}
$$

This factor is applied event by event: the probabilities $P_{\text {flat }}$ and $P_{\text {weighted }}$ are calculated integrating the flat and weighted distributions over a small interval centered on the event $(p, \cos \theta)$.

The optimal weighted generator can be identified requiring the minimal statistical uncertainty on the number of selected $\bar{p}$, for a fixed number of generated events $\left(N^{G E N}\right.$ total events, $N_{i}^{G E N}$ per bin). Being $w_{i}$ the weight of the i-th bin, the constrain translates into: $\sum_{i}^{n} w_{i}=1$ and the effective number of selected events will be (after cross section weights $\sigma_{i}$ and bin efficiency $\epsilon_{i}$ ):

$$
N_{i}^{S E L}=\sigma_{i} \epsilon_{i} N_{i}^{G E N}=\sigma_{i} \epsilon_{i} w_{i} N^{G E N}
$$


To estimate the real contribution we need to unweight each bin and so the final number of background events will be:

$$
N^{F I N A L}=\sum_{i}^{n} \frac{N_{i}^{S E L}}{w_{i}}
$$

The variance of this quantity is given by the the sum of the squared contributions:

$$
\operatorname{Var}\left(N^{F I N A L}\right)=\sum_{i}^{n} \frac{\operatorname{Var}\left(N_{i}^{S E L}\right)}{w_{i}^{2}}=\sum_{i}^{n} \sigma_{i}^{2}\left(N^{G E N}\right)^{2} \operatorname{Var}\left(\epsilon_{i}\right)
$$

assuming a binomial distribution for the $\epsilon_{i}: \operatorname{Var}\left(\epsilon_{i}\right)=\frac{\epsilon_{i}\left(1-\epsilon_{i}\right)}{N_{i}^{G E N}}=\frac{\epsilon_{i}\left(1-\epsilon_{i}\right)}{w_{i} N^{G E N}}$ expression in the 4.26 is equivalent to:

$$
\operatorname{Var}\left(N^{F I N A L}\right)=\sum_{i}^{n} \frac{\sigma_{i}^{2} \epsilon_{i}\left(1-\epsilon_{i}\right)}{w_{i}} N^{G E N}
$$

The problem now reduces to finding the values of $w_{i}$ for which the function 4.27 reaches its minimum, with the constrain $\sum_{i}^{n} w_{i}=1$. This can be solved with the usage of the Lagrange multiplicators and the result is:

$$
w_{i}=\frac{\sigma_{i} \sqrt{\epsilon_{i}\left(1-\epsilon_{i}\right)}}{\sum_{i}^{n} \sigma_{i} \sqrt{\epsilon_{i}\left(1-\epsilon_{i}\right)}}
$$

The denominator is a normalization common to all the bins and it is not relevant for the generator. The final result is that the best weights are given by:

$$
w_{i}=\sigma_{i} \sqrt{\epsilon_{i}\left(1-\epsilon_{i}\right)}
$$

which for $\epsilon<<1$ reduces to:

$$
w_{i} \simeq \sigma_{i} \sqrt{\epsilon_{i}}
$$

To obtain a reliable estimate of the $\mathrm{S} 1$ bin efficiencies $\epsilon_{i}$ from which infer the final weights, a preliminary simulation with $2 \times 10^{9}$ POT and a flat generator has been performed. Fig. 4.8 shows the distribution in generated variables $\cos \theta_{\text {gen }}$ and $p_{\text {gen }}$ for those events reaching the the entrance of the Transport Solenoid. Cross-section weights are not applied yet, so the z-axis / $N_{\text {generated }}$ corresponds to the geometrical bin acceptance expressed in terms of the generated variables. This preliminary study shows large inhomogeneities (up to $10^{4}$ ) in the phase space of the produced $\bar{p}$ s. Because of the $\sqrt{\epsilon_{i}}$ dependence of the generation weights in Eq. 4.29, the regions with higher efficiency must be enhanced, while it's not worth to waste time generating events in the regions where the acceptance is low.

The factor $\sigma_{i}$ in Eq. 4.29 is the integral of the cross section over a small bin in $(p, \cos \theta)$ (as it is for the unweighting probabilities). Fig. 4.9 shows the distribution of $\sigma_{i}$ for the two cross section models. The white region in Striganov's plot is an artifact: the bins with an occupancy below the experiment sensitivity are set to 0 .

The most challenging aspect of the antiproton background simulation is that some very low acceptance regions of Fig. 4.8, namely the ones corresponding forward production $\left(\cos \theta_{M u 2 e} \lesssim-0.5\right)$ at $p \sim 2 \mathrm{GeV} / \mathrm{c}$ momentum, are hugely enhanced by the cross section weights. This enhancement makes not possible to neglect these regions in the calculation of the final number of background events. Being the acceptance in these regions very low, a large fraction of the generated events will inevitably be wasted. 


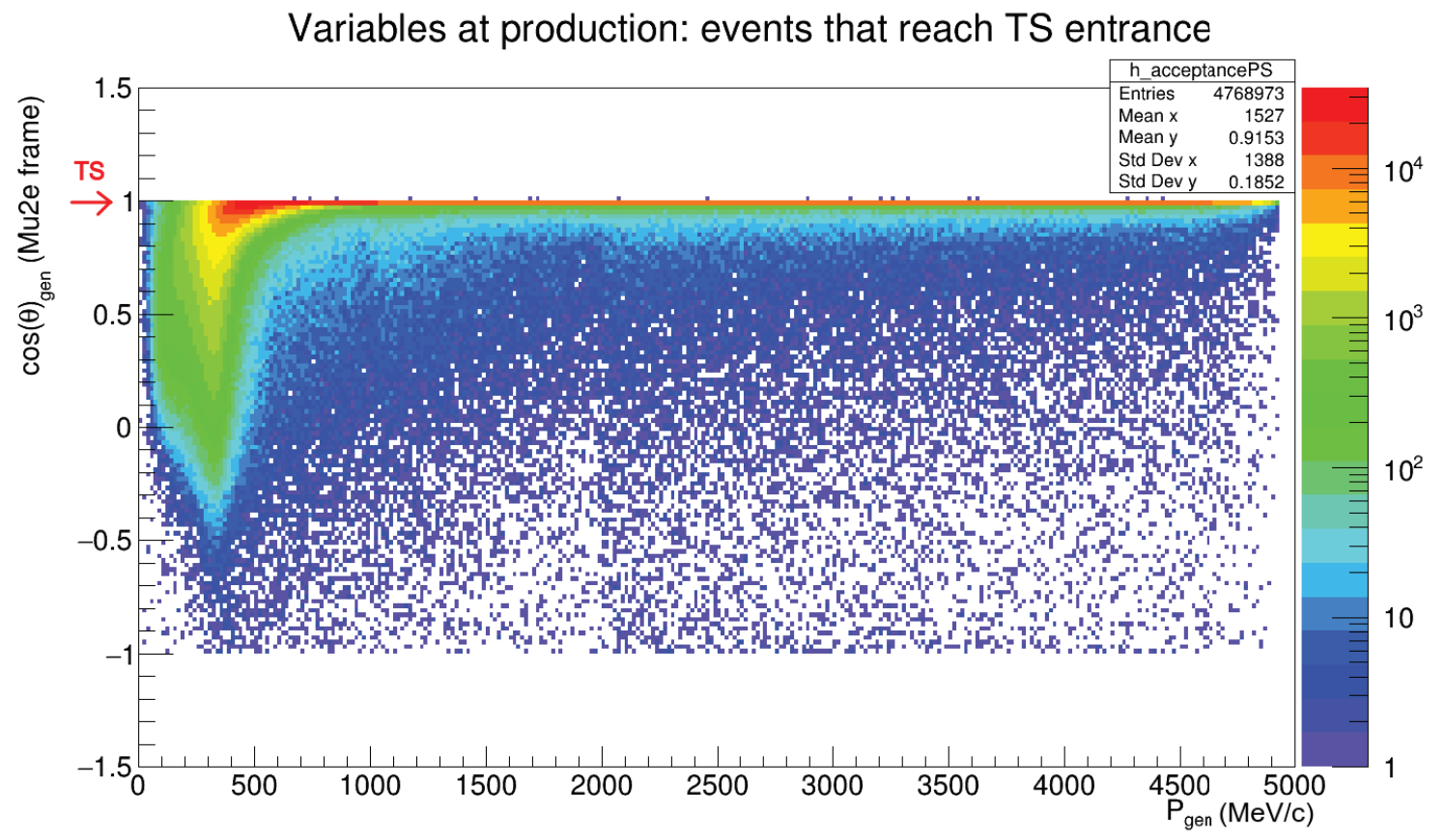

Figure 4.8: Distribution in the generated variables for events in the simulation that reach the VD at the entrance of TS. This preliminary simulation was implementing a flat generator so the plot also show the $p_{g e n}$ and $\cos \theta_{\text {gen }}$ dependence of the S1 efficiency.

The weights used for the weighted generator are obtained by the analytic expression:

$$
f(p, \cos \theta)=c_{1} \cdot e^{-c_{2} \cdot p \cdot(\cos \theta+1)}+e^{-c_{3}(\cos \theta+1)} \cdot \operatorname{Gaus}(p, 2 G e V, 0.7 G e V)
$$

with:

$c_{1}=10^{-6}$

$c_{2}=5 \cdot 10^{-3}$

$c_{3}=17$

This approximates the dependence on $p$ and $\cos \theta$ of $\sigma \times \sqrt{\epsilon}$ obtained combining the $\sigma$ Bernstein's model ${ }^{9}$ and the S1 acceptance ${ }^{10}$ given by the flat generator. The quality of the approximation is shown in Fig. 4.10 where the original weights distribution (left) is compared to the one obtained using the analytic expression for the random extraction (right): the agreement is considered sufficient to guarantee the wanted CPU time saving.

The $\mathcal{P}$ (weighted) factor in 4.23 is obtained numerically integrating the analytical weight function in a small bin around the event. The size of this bin (and the one used for the calculation of $\sigma_{i}$ ) is much smaller than the binning shows in the plots of this chapter.

In summary the weighted generator works as follows:

\footnotetext{
${ }^{9}$ This is an arbitrary choice: it's not needed to reproduce $\sigma_{i}$ in great detail, given also the discrepancies observed between the two models, it's more important to have a regular function that can be easily integrated without introducing numerical biases. Also a $\sqrt{s}$ dependence, which is a-priori needed to reproduce the $\sigma_{i}$ correctly, is not included in the analytical function

${ }^{10}$ The acceptance of the stages following S1 is much less dependent on the antiproton generated variables. On the other hand using S1 acceptance allows to have a reasonable amount of events for the acceptance calculation in an affordable time.
} 

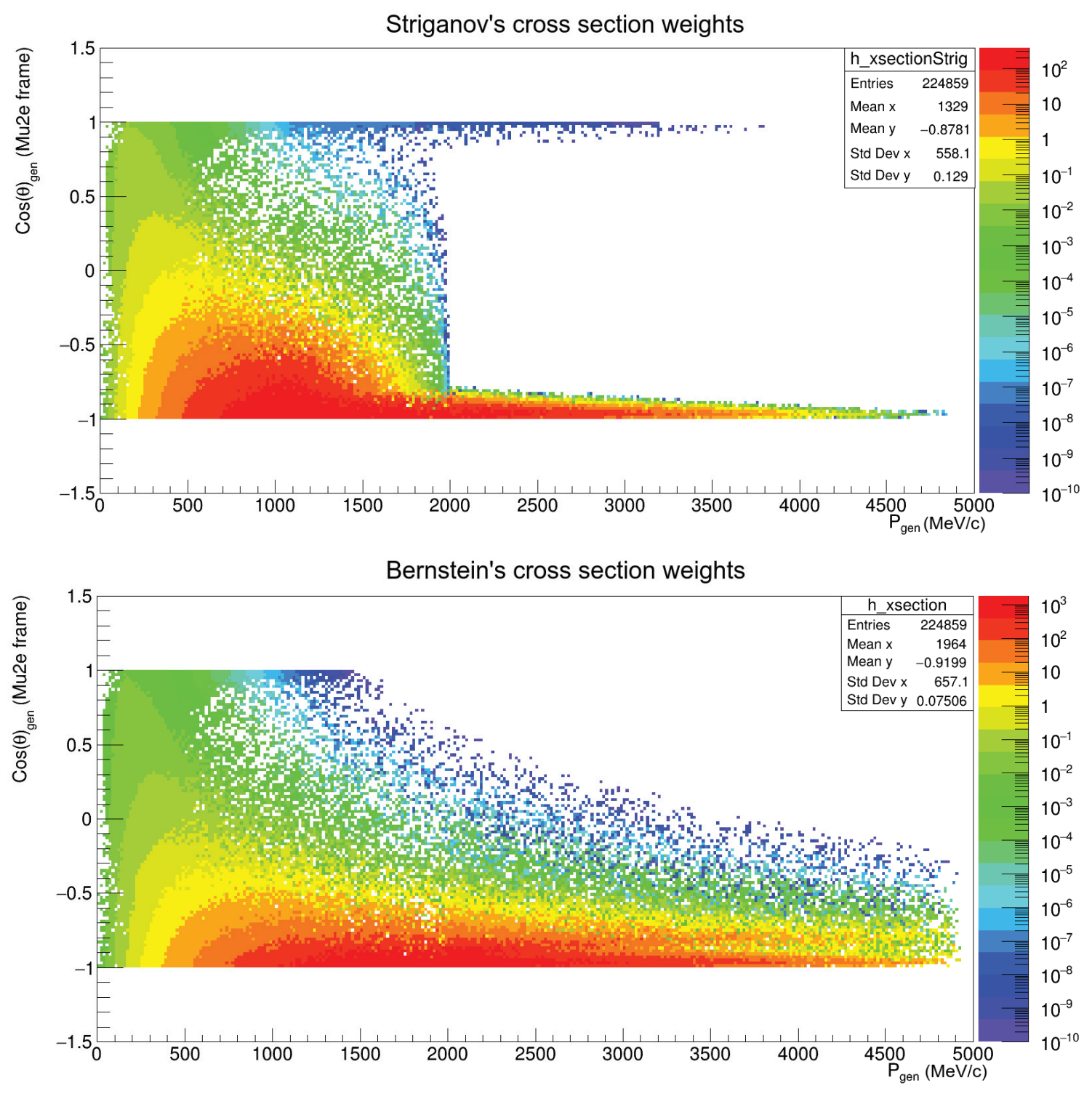

Figure 4.9: Cross section weights, with $\sqrt{s}$ given by interacting protons in the PT (energy losses included), to be applied to each bin according to Striganov's (TOP) and Bernstein's model (BOTTOM). The bin size is $16.5 \mathrm{MeV} / \mathrm{c} \times 0.02$.

- for each S0 vertex the value of $\sqrt{s}$ associated to the incident proton momentum ${ }^{11}$ is calculated assuming only one proton as target (no multi-nucleon interaction);

- the maximum momentum allowed in the CM follows from Eq. 4.6;

- Lorentz boost is applied to obtain the maximum momentum in the LAB frame, $p_{M A X}$. Typical values are always below $5 \mathrm{GeV} / \mathrm{c}$.

- the momentum is extracted from 0 to $p_{M A X}$ and $\cos \theta$ from -1 to 1 using the $2 \mathrm{D}$ histogram in Fig. 4.10.

\footnotetext{
${ }^{11}$ The proton momentum is the one at the vertex so that the eventual energy loss or multiple scattering occurring in the PT before the interaction are taken into account.
} 

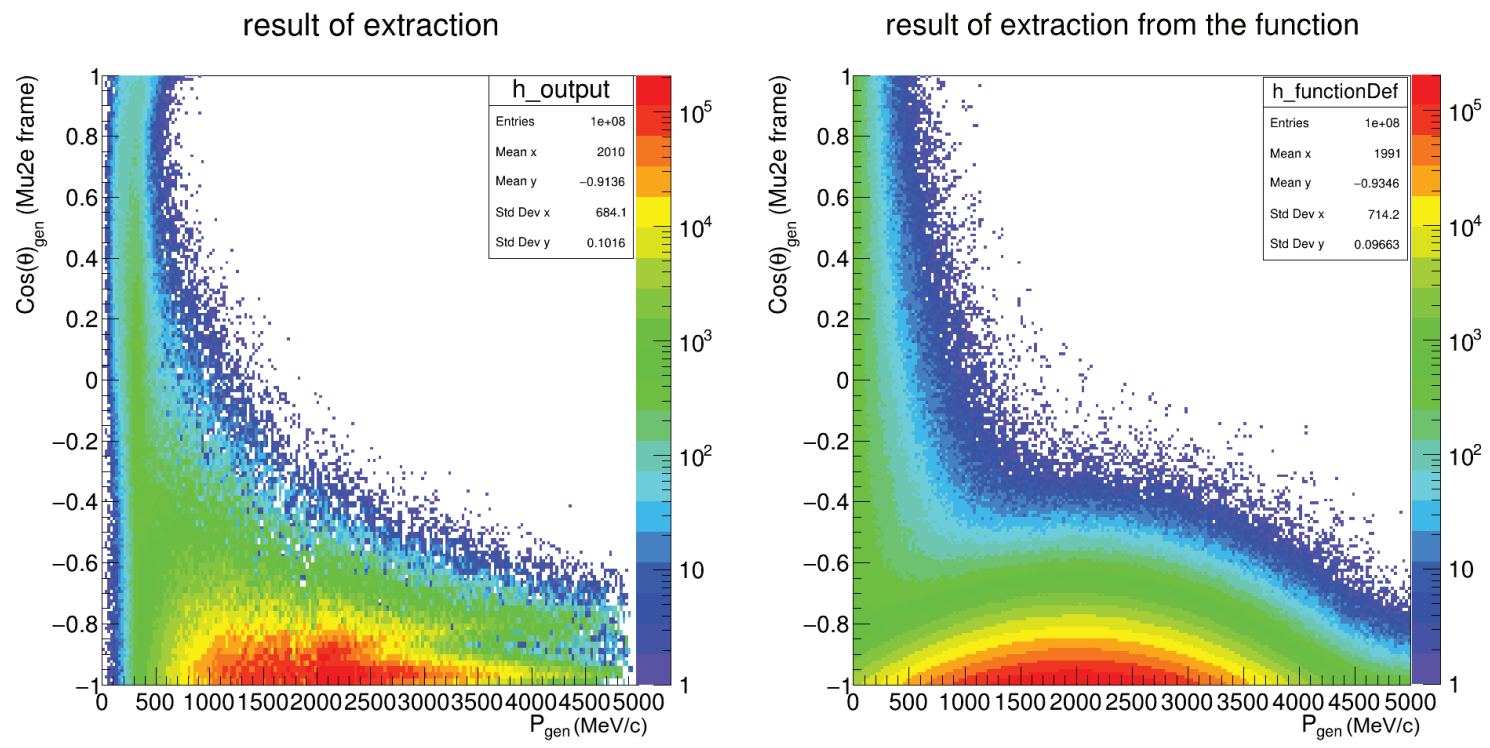

Figure 4.10: Distribution of $\sqrt{\epsilon_{i}} \times \sigma_{i}$ at the of S1 obtained using a flat generator (left). On the right the distribution of the same variable but randomly extracted using the analytical approximation described in the text.

The values of the generated variables are accessible at each stage of the generation so that the weight factors needed to produce the unweighted distributions (Eq. 4.23) and cross-section can be calculated at any time.

\subsubsection{Tracing inside the PS}

After their generation, S1 antiprotons are traced out of the Production Target and inside the Production Solenoid. Only events that reach virtual disk placed in front of the aluminum absorber right before the entrance of the TS are saved.

Accepted events can be grouped in three main categories :

- Antiprotons generated directly towards the TS $\left(\cos \theta_{M u 2 e} \simeq 1\right)$ for which the acceptance is independent on the generated momentum magnitude. Their acceptance is very high but on the contrary their production probability is very low since they are produced in the direction opposite to the incident proton momentum $\left(\cos \theta_{X s e c} \simeq-1\right)$ 12 : the cross section decreases very fast with momentum making the contribution of events with $p>800 \mathrm{MeV} / \mathrm{c}$ negligible.

- Low momentum $\bar{p}$ s generated at wider angles. If they are produced backwards $\left(\cos \theta_{X s e c}<0\right)$ their momentum must be such that their trajectory crosses the virtual detector located at the entrance of the TS, represented by the white circle in Fig. 4.11. If they are produced forward $\left(\cos \theta_{\text {Xsec }}>0\right)$ their momentum must be low enough that the magnetic field gradient is able to reflect them back towards the TS 13 .

\footnotetext{
${ }^{12}$ The backward direction in fact corresponds to a $\cos \theta_{X \text { sec }}$ slightly higher than -1 because of the $14^{\circ}$ inclination between the Mu2e and Xsec frames (see Fig. 4.1).

${ }^{13}$ The effect, known as "magnetic mirror", originates from the energy and angular momentum conservation in the adiabatic approximation.
} 


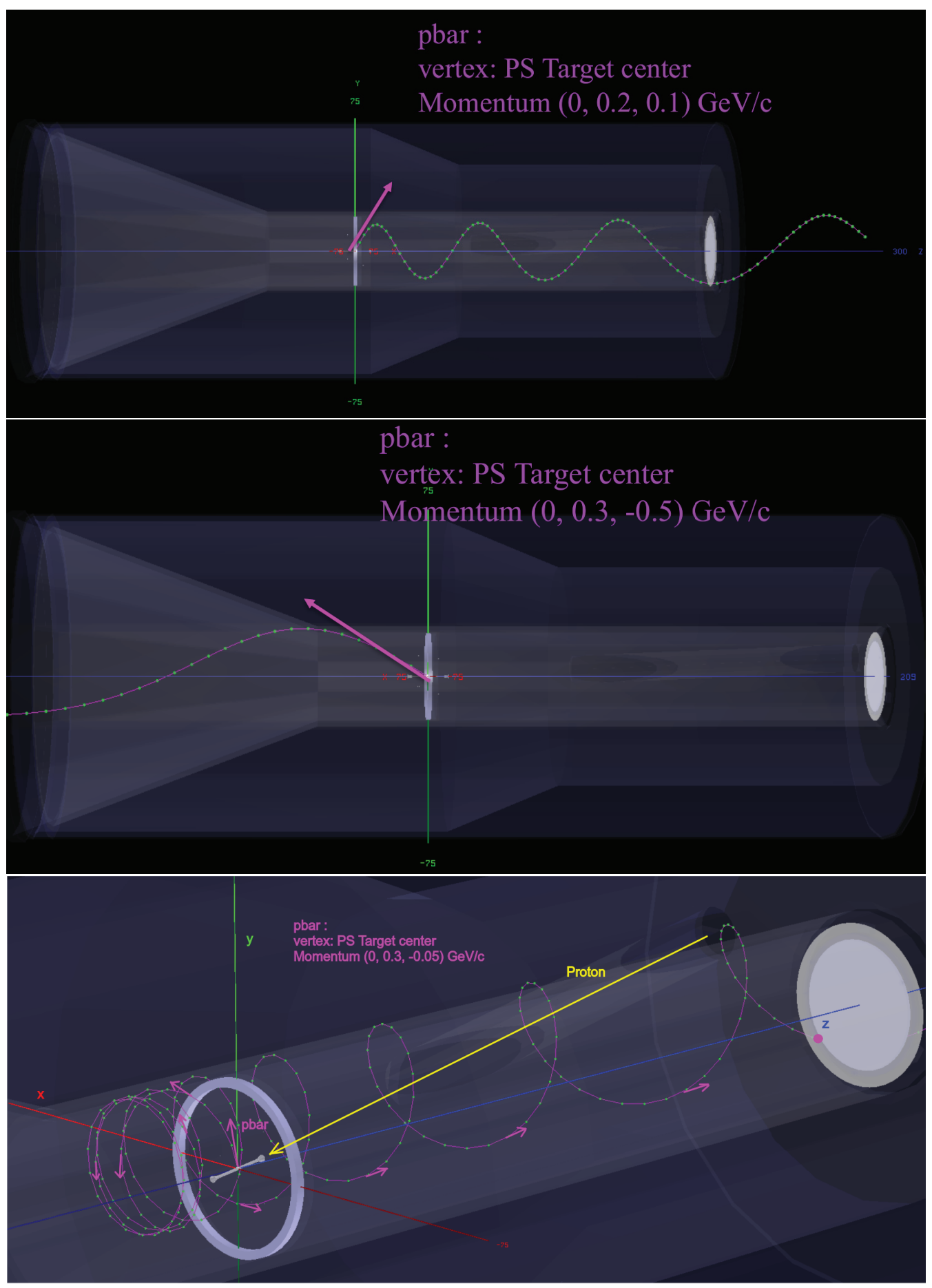

Figure 4.11: Examples of events reaching or not the TS: a backward produced $\bar{p}$ that directly travels to the TS (accepted); a forward produced $\bar{p}$ with high momentum that proceeds in the forward direction (not accepted) and a forward produced low momentum $\bar{p}$ reflected by the magnetic field gradient towards the TS (accepted).

- High momentum, forward produced antiprotons which undergo an inelastic backscattering interaction inside the Production Target. The interaction is driven by G4 
using the same model used for hadron interactions ${ }^{14}$ : the antiproton excites the nucleus that then deexcites producing nucleons, nuclear fragments and a backward going antiproton with considerably smaller momentum.

Events in this category have a limited geometrical acceptance but are heavily enhanced by the cross section so that their contribution to the final background appears to be of the same order of the one of the previous two categories. This result critically relies on the model Geant 4 uses for the low momentum $\bar{p}$ back-scattering that cannot be easily validated given the lack of experimental data. One of the merit of this study has been the one to clarify this problem to the Mu2e collaboration. Further investigations, including also the possibility for an in situ measurement of the $\bar{p}$ background, will be needed.

\subsubsection{Results after Stage 1}

The inelastic interaction vertices produced by $N_{\text {sim }}=10^{10}$ proton on targets have been produced by the stage 0 . The weighted generation has then been used to generated one antiproton from each stage 0 vertex. The antiprotons have then been traced through the PS and only the ones reaching the TS have been recorded. Each event has been unweighted by the generator weight in Eq. 4.23 and re-weighted with the correct production probability obtained from the cross section model. Fig. 4.12 shows distribution in $p$ and $\cos \theta$ of the events reaching the TS, obtained using Bernstein's cross section. It is evident how, when

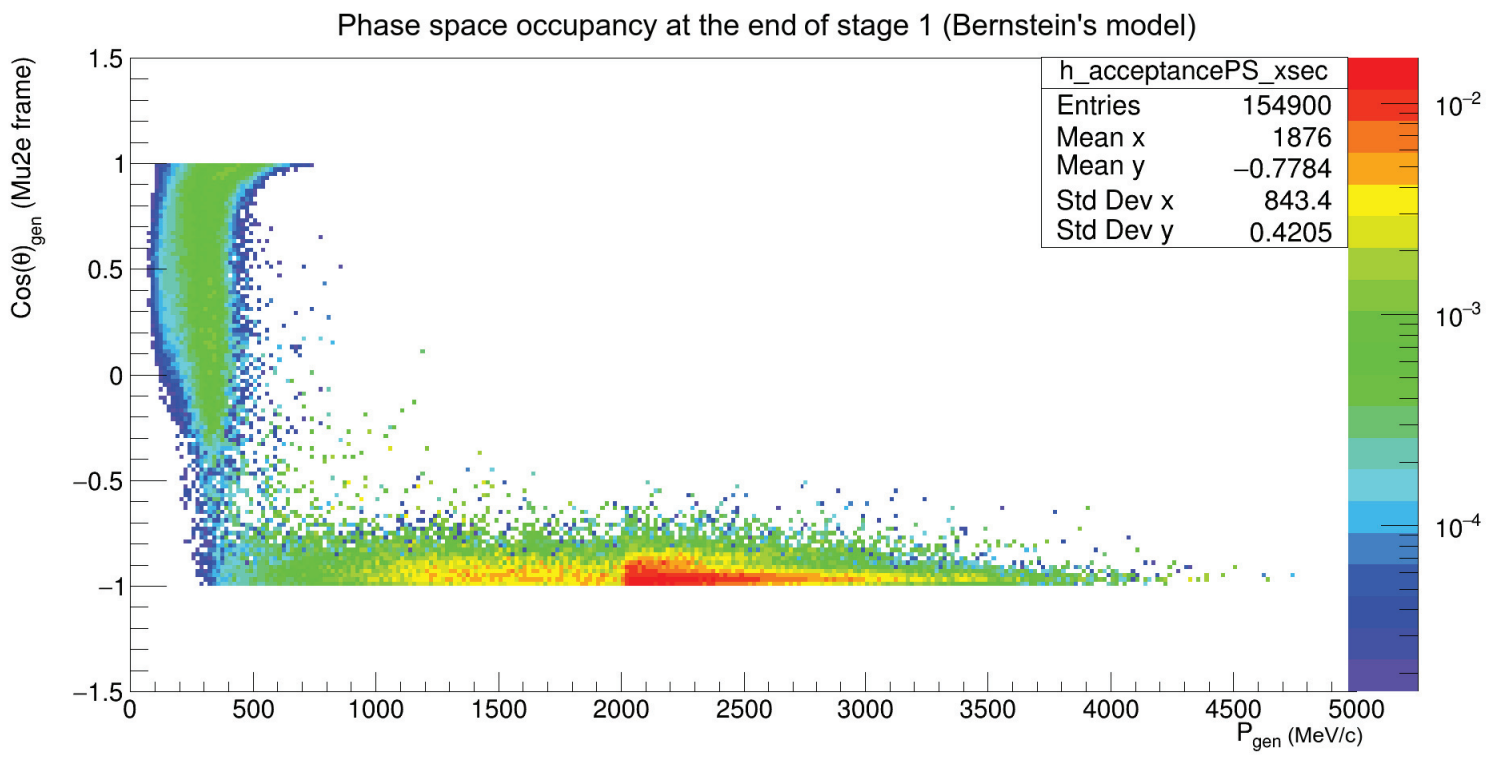

Figure 4.12: 2D distribution in the generated variables for the events entering the TS (end of S1), after unweighting for the weighted generator weights and applying Bernstein's cross section weights. Statistical uncertainties are higher in regions disfavored by the generator.

the cross section is taken into account, regions highly disfavored by the efficiency (Fig. 4.8) become dominant.

Fig. 4.13 shows the distributions of $\cos \theta$ and $p$ using the two cross section models. Looking at the momentum distribution, three different regions can be identified corre-

\footnotetext{
${ }^{14}$ The G4 ShieldingM physics list has been used.
} 

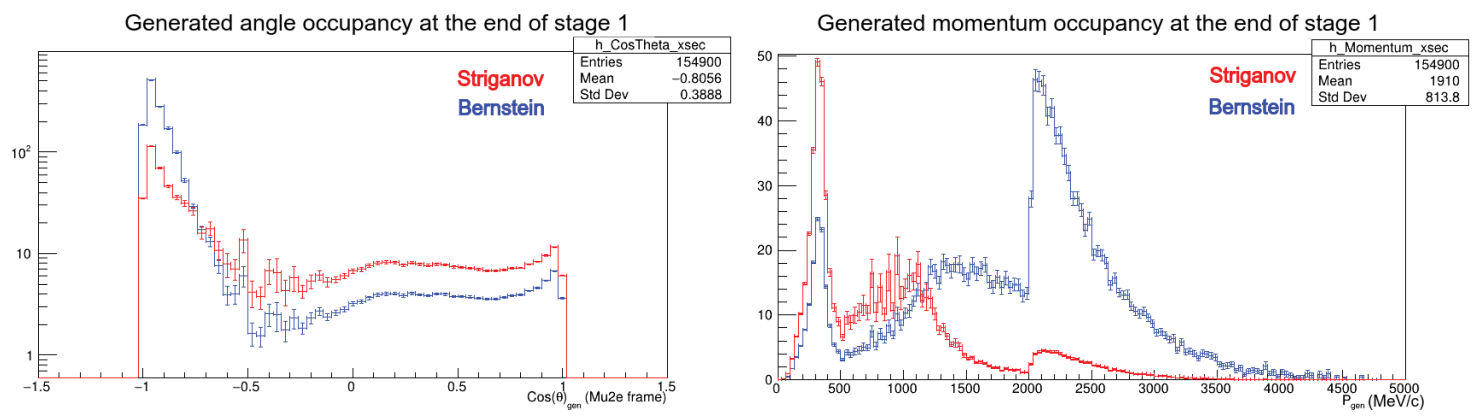

Figure 4.13: $\cos \theta_{M u 2 e}$ and $P$ projections of the phase space distribution from Striganov's and Berstein's model. Statistical uncertainties are higher in regions disfavored by the generator.

sponding to the event categories discussed in Sec. 4.4.2: one prominence at $\cos \theta \sim 1$ that is the the remnant of the convolution between the high-acceptance tail visible in Fig. 4.8 and the backward production suppression; a low momentum region that includes both forward and backward emitted $\bar{p}$; a high momentum region $(p>2 \mathrm{GeV} / \mathrm{c})$ concentrated at low emission angles. The last category correspond to the $\bar{p}$ back scattering from the nucleus discussed at the end of the previous paragraph. The peak observed at $1.2 \mathrm{GeV} / \mathrm{c}$ for Striganov's distribution is not so pronounced in Berstein's one and is then probably an effect of the combination of the cross section convolution with the acceptance. The contribution from the region $p>2 \mathrm{GeV} / \mathrm{c}$ and $\cos \theta_{M u 2 e} \sim-1$ is much more relevant in the case of Berstein's model.

\subsection{S2: from TS entrance to COL3u exit}

Stage 2 operates the event tracing through the first three sections of the TS until a VD placed at the end of the first half of the TS (COL3u), right before the titanium absorber window (Fig. 4.14).

The $\bar{p}$ trajectory is determined by its position and momentum at the entrance of the TS and keeps no memory of the properties of the $\bar{p}$ generated in the production target. Fig. 4.15 shows the momentum distribution of the $\bar{p}$ at the entrance of TS: the maximum momentum is $\sim 500 \mathrm{MeV} / \mathrm{c}$.

The TS geometry is especially designed to suppress backgrounds related to the beam. This is particularly important for antiprotons, given the presence of special absorber elements and collimators.

Starting from this stage (S2) ${ }^{15}$, also the pions coming out from antiproton interactions are traced and propagated to the next stage.

\subsubsection{The resampling technique}

A preliminary study of the efficiency of stage 2 and 3 has showed that even $10^{10}$ POT produce very few events reaching the end of the TS. An event resampling technique can solve the problem without increasing the needed computing resources to an unaffordable level.

\footnotetext{
${ }^{15}$ Pions coming out of the Production Target are instead traced in the ordinary estimate of the RPC component and must not be counted twice.
} 


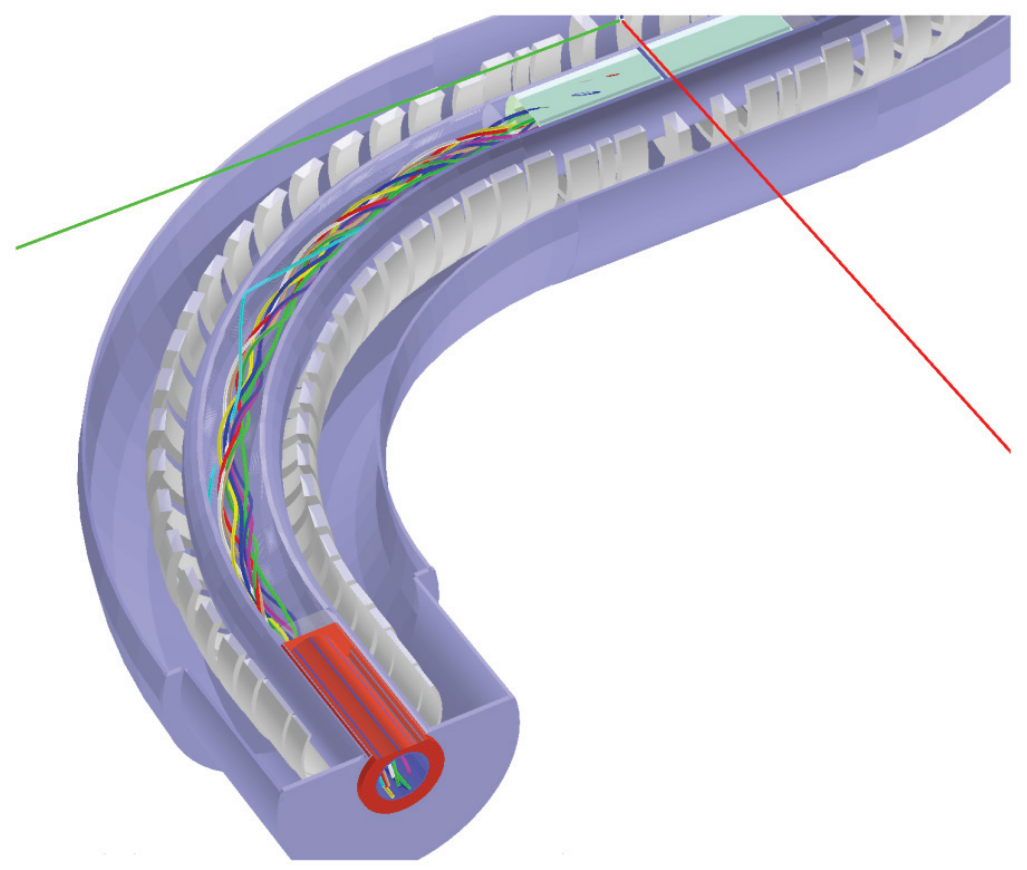

Figure 4.14: Examples of propagation inside the TS2: accepted particles enter from the bottom and reach the TS3 (cyan cylinder) on the top. Each color corresponds to a different track.

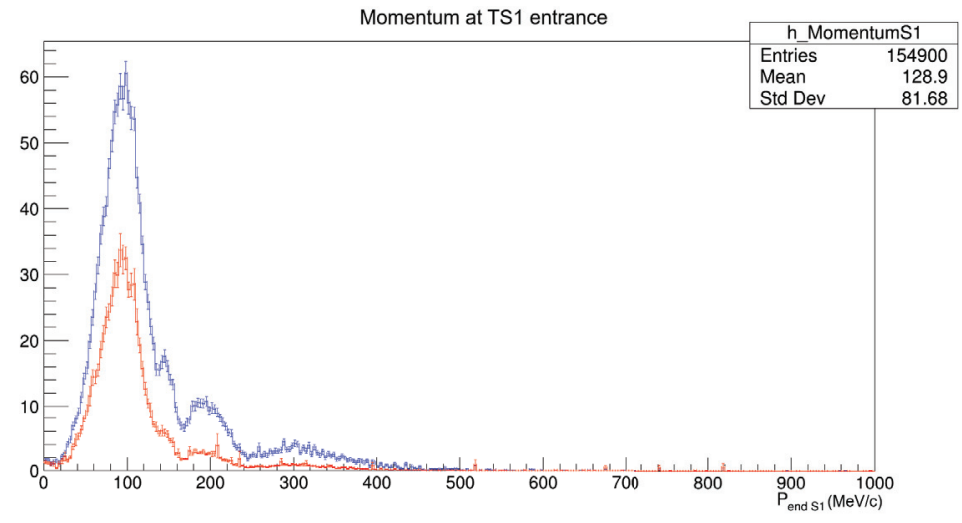

Figure 4.15: Momentum of the $\bar{p}$ reaching TS entrance (end of S1 and start of S2).

The resampling consists of the fact that each $\bar{p}$ reaching TS entrance can be reused multiple times to obtain many different events. The events will in fact be different thanks to the statistical fluctuation of energy loss and multiple scattering suffered by the $\bar{p}$ while crossing the TS absorbers and collimators. The number of times each event is reused is called resampling factor.

Two important conditions must be realized for the resampling technique to work:

- the initial population must be representative of the whole phase space otherwise the regions not represented will not be present in the final sample;

- the ideal resampling factor should be such that no more than one event per original event will pass the final selection, otherwise we have the so called overtraining. In 
practice a low fraction of events in the final sample coming from the same original event can be considered acceptable.

Pathologies due to the oversampling appear as spikes and inhomogeneities in the histograms. A resampling factor of $10^{5}$ at the stage 2 has shown to produce a negligible oversampling. The possibility to use the resampling technique at stages later than 2 has also been studied but has been rejected because of the large oversampling observed in the final sample ${ }^{16}$.

\subsection{S3: from TS center to TS5 entrance}

Subsequent tracking from the exit of collimator COL3u to the last straight section of TS (TS5) is handled by the stage 3 . The most relevant part of this simulation is the transportation through the titanium absorber and the two Al foils suspended between COL3u and COL3d (see Sec. 2.3.2).

Fig. 4.16 shows the $\bar{p}$ momentum distribution before and after the absorber : the events with $p<100 \mathrm{MeV} / \mathrm{c}$ are completely suppressed.
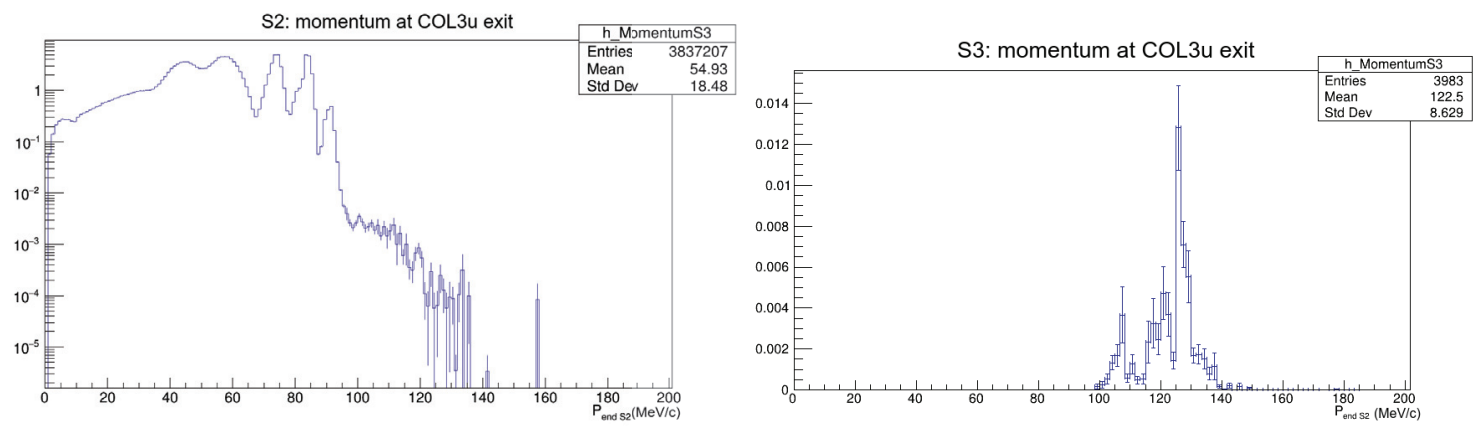

Figure 4.16: (LEFT) Momentum distribution of the antiprotons reaching the titanium absorber (S2 end). Bernstein's cross section weights have been applied. (RIGHT) Same distribution (momentum at end of S2) for the $\bar{p}$ s surviving the absorber.

\subsection{S4: from TS5 entrance to the Stopping Target}

The last obstacle encountered by the antiprotons before the DS is the collimator located in the last section of the TS (COL5).

Not all the particles entering the DS intersect the Stopping Target: the inner hole of COL5 is bigger than the ST foils $\left(R_{C O L 5}=128 \mathrm{~mm}\right.$ vs $\left.R_{S T}=77 \mathrm{~mm}\right)$ and the magnetic field gradient pushes out the particles with high transverse momentum. As a result only $55 \%$ of the antiprotons reaching the TS5 entrance are stopped in the ST.

Fig. 4.19 shows the z-coordinate of the antiprotons stopped in the ST: most of the stops occur in the very first $\mathrm{Al}$ foils.

The following paragraphs present the results obtained combining the output of two different generation campaigns: in the first $10^{10}$ POT have been simulated at S0, a flat momentum generator and an exponential weighted generator enhancing forward production have been used at S1 and a resample factor of $5 \times 10^{4}$ has been used at $\mathrm{S} 2$; in the second

\footnotetext{
${ }^{16}$ This can be explained considering the lower quantity of material crossed by the particles after stage 2.
} 
$10^{10}$ POT have been simulated at S0, a weighted generator has been used at S1 and a resampling factor of $10^{5}$ has been applied at S2. The first generation resulted in 1315 stopped $\bar{p}$, while the second one has produced 968 stops with a better covering of the critical region where back-scattered $\bar{p}$ s contribution is relevant. The two data sets have been combined using the appropriated weights from Eq. 4.23.

\subsubsection{Origin of stopped $\bar{p}$ events}

What is the path followed by the antiprotons stopping in the ST? To answer this question we can analyze the position and momentum of these antiprotons at the end of the previous stages.
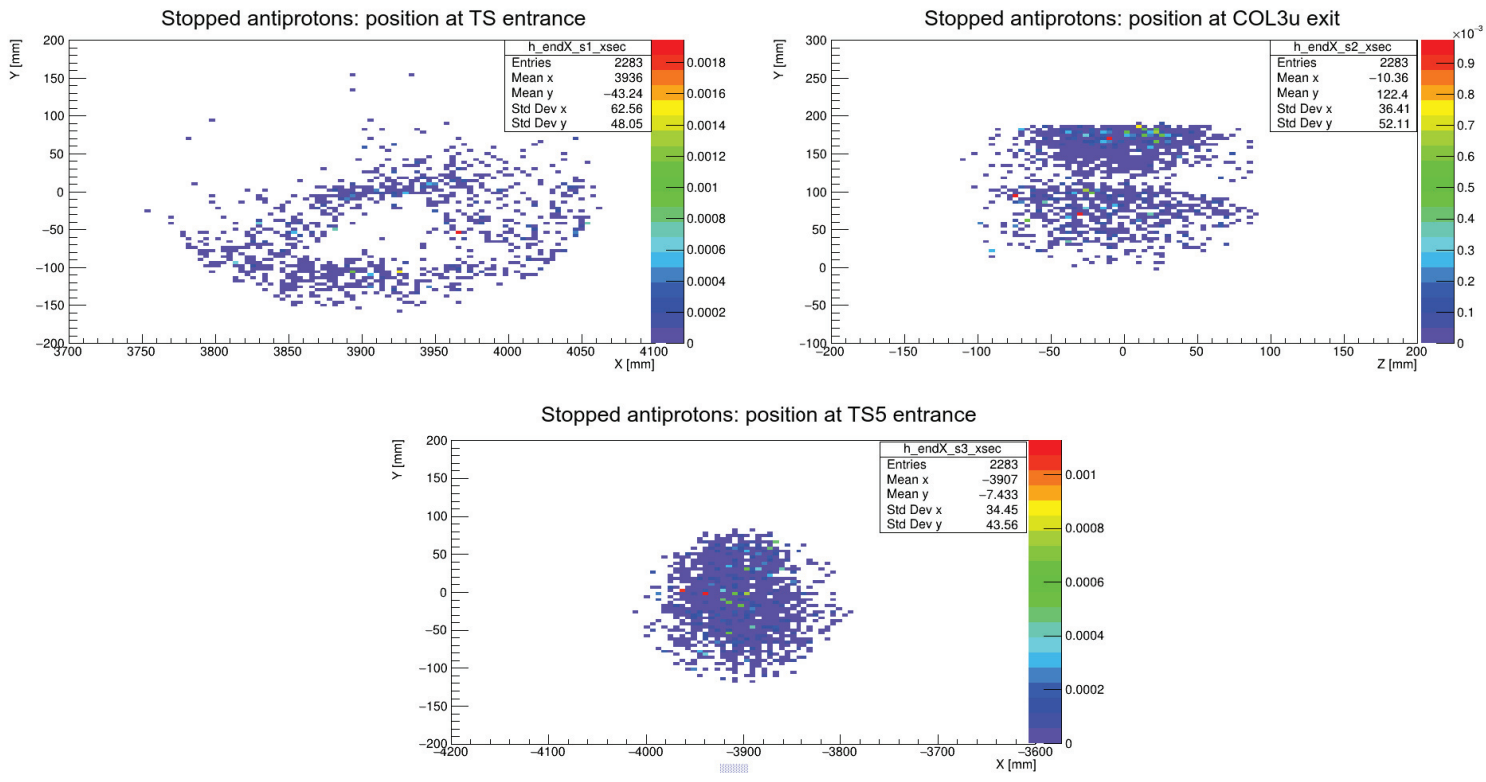

Figure 4.17: Position at the end of S1 (TOP LEFT), S2 (TOP RIGHT) and S3 (BOTTOM) for the antiprotons stopped in the ST. Cross-section weights from Bernstein's model have been applied.

Fig. 4.17 shows the position of the stopped $\bar{p}$ s at the end of S1, S2 and S3. Bernstein's cross section has been used to produce the correct event probability ${ }^{17}$. On the top left plot it's possible to note how the center of gravity of the distribution at the entrance of TS is shifted towards negative Y values: this is the reason why the TS1 arc collar has been introduced. The collar, that extends for $20 \mathrm{~cm}$ along $\mathrm{z}$, is also responsible for the hole observed in the distribution.

The top right pictures shows the position of the stopped $\bar{p} \mathrm{~s}$ at the exit of COL $3 \mathrm{u}$, that is just before the titanium absorber window located at the center of TS. The region populated by the events corresponds to the COL3u hole ${ }^{18}$ and is shifted towards positive Ys: this has brought to the introduction of the two $\mathrm{Al}$ suspended foils after the Ti absorber.

\footnotetext{
${ }^{17}$ The choice of the cross section model is not so relevant at this point because the features observed in the distributions are mostly determined by the geometry of the absorber material crossed by the antiprotons along their path.

${ }^{18}$ In fact the COL3u can be rotated to select positive or negative particle: all the simulation has been performed using the negative particle selection configuration.
} 
The distribution at TS5 entrance (bottom plot) is much more uniform and corresponds to the opening of the COL5 collimator.

Fig. 4.18 shows the momentum distribution of the stopped $\bar{p}$ at the end of S1, S2 and S3. Also in this case Bernstein's cross section weights have been applied.
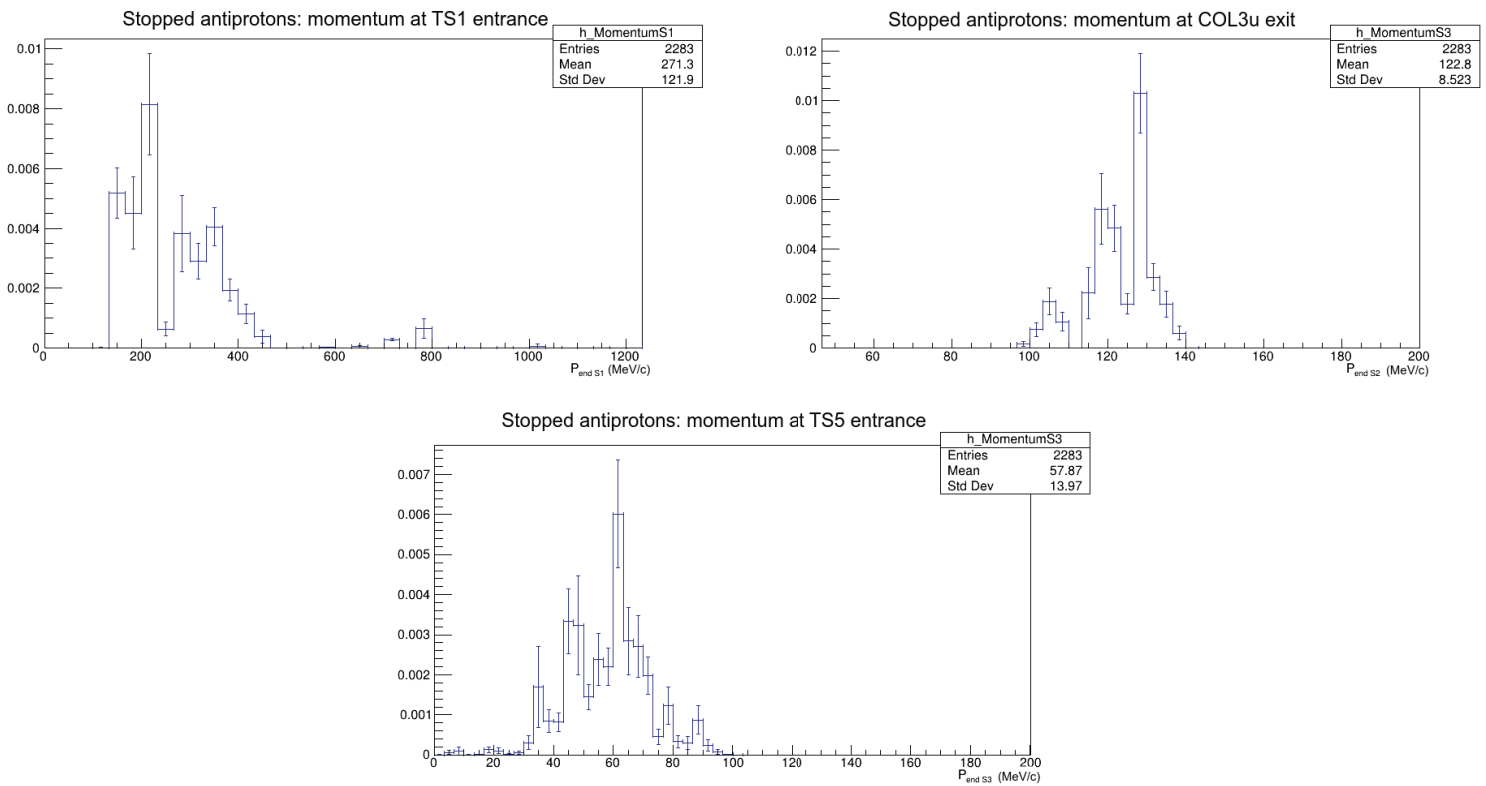

Figure 4.18: Stopped $\bar{p}$ s momentum distribution at the end of stage 1 (TOP LEFT), stage 2 (TOP RIGHT) and stage 3 (BOTTOM), shown for those events who stop inside the ST. Cross-section weights from Bernstein's model have been applied.

The top left plot shows that the great majority of the events reaching TS entrance have a momentum between 100 and $450 \mathrm{MeV} / \mathrm{c}$ : higher momentum events are exponentially suppressed by the cross section and practically not visible in the plot given the available statistics.

The distribution at the end of the stage 2 (top right plot) is shifted towards lower momenta because of the energy loss in the TS1 absorbers. Everything that falls below $100 \mathrm{MeV} / \mathrm{c}$ is lost because absorbed in the following titanium window. The effect of the titanium window and of the $\mathrm{Al}$ foils is to further reduce the momentum so that no antiprotons with momentum higher than $100 \mathrm{MeV} / \mathrm{c}$ reach the TS5 entrance (bottom plot).

\subsubsection{Position of stopped antiprotons in the Stopping Target}

Fig. 4.19 shows the positions where antiprotons stop in the Stopping Target.

Antiprotons with $p<100 \mathrm{MeV} / \mathrm{c}^{19}$ most likely stop in the very first Al foils. Anyway, recorded stops extend for the total length of the ST (approximately $800 \mathrm{~mm}$ ) and allow to create a reliable distribution for the $\bar{p}$ annihilation vertices.

\footnotetext{
${ }^{19}$ See previous paragraph.
} 

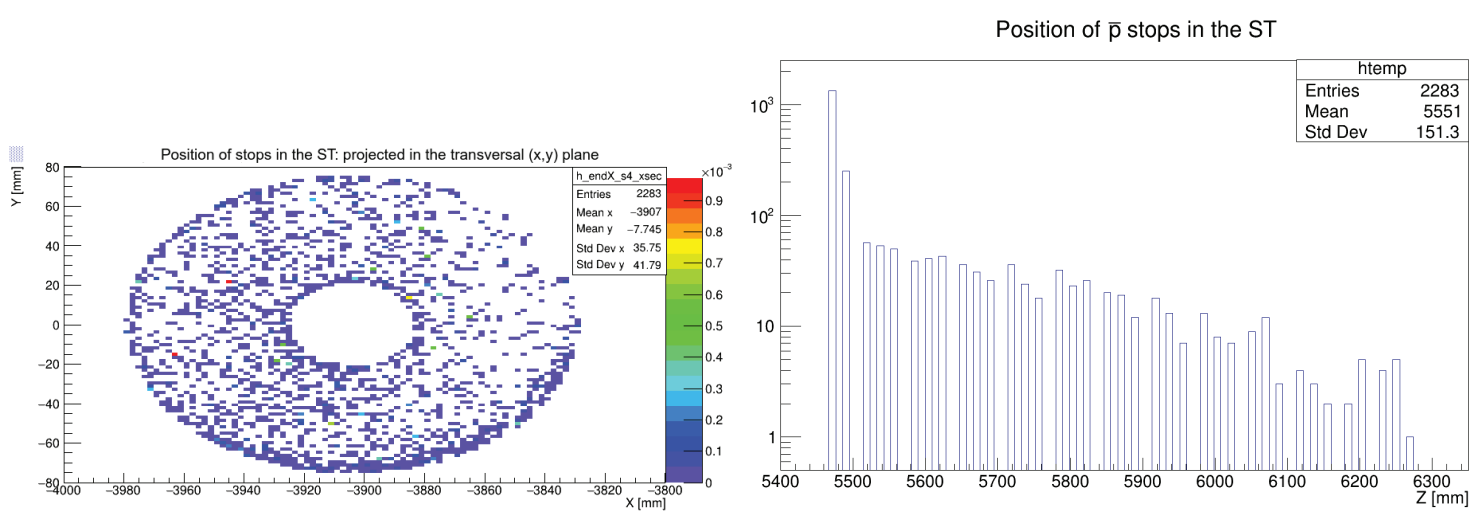

Figure 4.19: Position of the $\bar{p}$ stops in the X-Y plane (LEFT) and along Z (Right). The single ST foils are visible.

\subsubsection{Number of stopped $\bar{p}$ per proton on target}

Fig. 4.20 shows the distributions in the generated variables for the antiproton stopped in the ST. If the events were generated according to the probability given by the differential
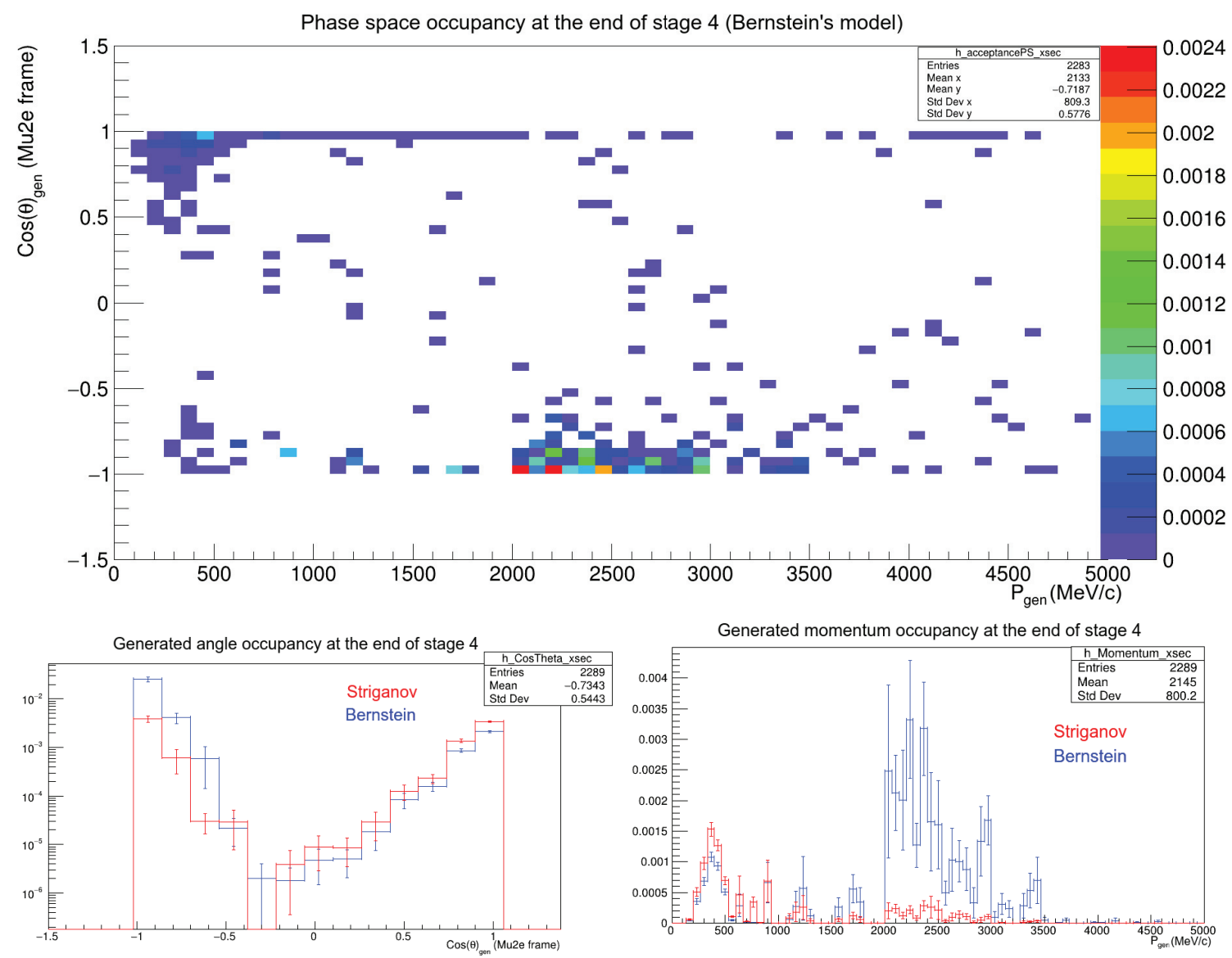

Figure 4.20: (TOP) 2D distribution of the generated variables for the antiprotons stopped in the ST. On the bottom: $\cos \theta$ (LEFT) and momentum (RIGHT) projections for the same events.

cross section, the sum of the entries in the histogram would already provide the correct 
number of stopped $\bar{p}$ s for the generated number of POT.

In fact, since the event have been generated using a weighted generator, the entries of the i-th bin must enter into the sum with a weight that restores the correct probability for the bin:

$$
N_{\text {stops }}=\sum_{\text {bins }} N_{i} w_{i}
$$

where $N_{i}$ is the number of entries in the bin and $w_{i}$ is the bin weight ${ }^{20}$ :

$$
w_{i}=\frac{P_{\text {flat }}}{P_{\text {weighted }}} \frac{P_{\sigma}}{P_{\text {flat }}}=\frac{P_{\sigma}}{P_{\text {weighted }}}
$$

where $P_{\sigma}$ is the probability obtained from the differential cross section integrated over the bin:

$$
P_{\sigma}=\frac{\int_{b i n} \frac{d^{2} \sigma}{d p d \cos \theta}}{\sigma_{p A \rightarrow \bar{p} X}}
$$

and $P_{\text {weighted }}$ and $P_{\text {flat }}$ are the integral over the bin of the probability function used for the weighted and flat generator respectively.

All the integrals are calculated numerically.

Reminding the correction factor introduced in Eq. 4.21 to take into account the fact that not all the vertices used at the beginning of S1 correspond to a $\bar{p}$ production, the effective number of POT corresponding to the histogram statistics will be:

$$
N_{P O T}=N_{\text {sim }} \frac{\sigma_{p A \rightarrow \bar{p} X}}{\sigma_{\text {tot. inelastic }}}
$$

where $N_{\text {sim }}$ is the number of protons simulated at S0 multiplied by the resampling factor, that, considering the sum of the two production campaigns, is $N_{\text {sim }}=10^{15}+5 \cdot 10^{14}$ (resampling factors included).

Finally, the number of stopped antiprotons per POT is given by:

$$
\mathcal{P}(\bar{p} \text { stop. } \mid \text { POT })=\frac{N_{\text {stops }}}{N_{\text {POT }}}= \begin{cases}(0.65 \pm 0.04(\text { stat })) \times 10^{-17} & \text { (Striganov's model }) \\ (2.22 \pm 0.19(\text { stat })) \times 10^{-17} & \text { (Bernstein's model })\end{cases}
$$

The statistical uncertainty is estimated using the square root of the sum of the squared weights.

\subsubsection{Check of the resampling technique}

At this point it's possible to check if the resampling performed at S2 has an impact on the result.

Fig. 4.21 the number of times the same generated antiproton appears as original particle for an event of $\bar{p}$ stop in the Stopping Target. As discussed in Sec. 4.5.1 this number should ideally be 1 and in fact the mean of the distribution is just slightly more than 2.

To check how much the resampling affects the final result, the content of the bins in which more than 10 events originating from the antiproton were present has been replaced

\footnotetext{
${ }^{20}$ The intermediate passage is not necessary and has only been shown to keep track of the weights discussed in Eq. 4.23.
} 
by the mean of the adjacent bins. When this correction is applied the number of $\bar{p}$ stops changes by $1 \%$, that is a small variation with respect to the uncertainties quoted in Eq. 4.35 .

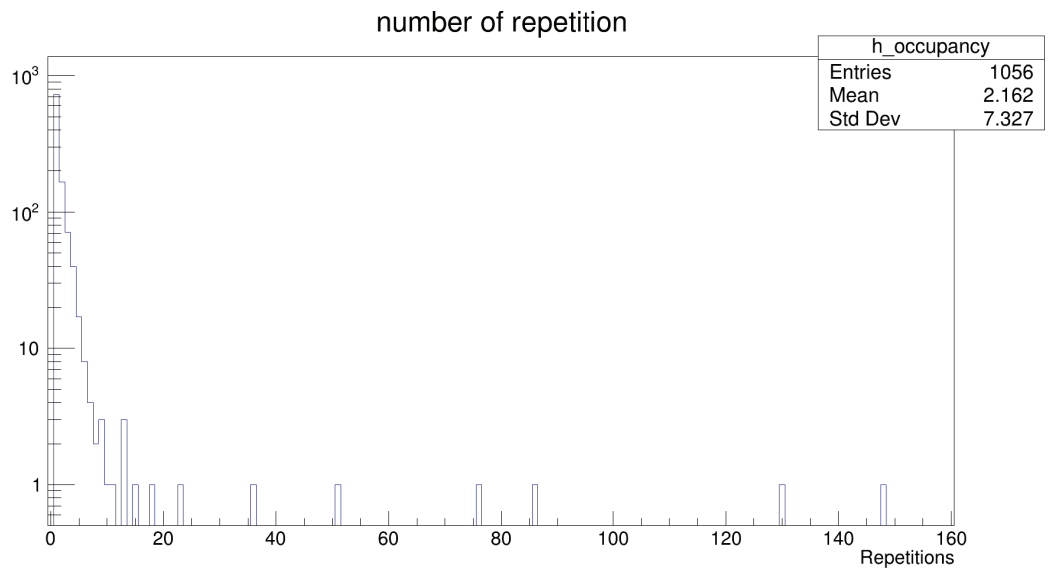

Figure 4.21: Number of times the same generated antiproton is repeated in the final sample of stopped $\bar{p}$ s. The majority of the events appears just once.

\subsection{S5: from the Stopping Target to the detectors}

Stage 5 is used to create the antiproton annihilations at rest in the Stopping Target using the position and time of the $\bar{p}$ stops recorded in the previous stage. Given the large variety of possible final states for the $\bar{p}$ annihilation the same $\mathrm{S} 4$ vertex can be reused many time (S5 resampling).

The $\bar{p}+p$ annihilation at rest is well supported by experimental data and its implementation in G4 is considered enough accurate to exclude the need for custom generators.

From the point of view of the CE background, the most dangerous secondary products of the annihilation are, in order of importance:

- $\pi^{0} \mathrm{~s}$ that immediately decay producing electron-positron pairs ${ }^{21}$ and photons which then convert into electron-positron pairs in the ST or inside walls of the tracker.

- $\pi^{-} \mathrm{s}$ that decay in muons that then decay into electrons.

All the particles produced in the annihilations are traced in the Detector Solenoid and the hits they produce in the detectors are stored. Only the events with at least 10 hits in the tracker are passed to the reconstruction stage.

A total of $3 \cdot 10^{7} \bar{p}$ annihilations in the ST have been generated and the efficiency of this stage is estimated to be $\epsilon_{S 5} \simeq 20 \%$.

This is the first time a complete simulation of $\bar{p} \mathrm{~s}$ in the Mu2e detector has been used to find their annihilation position and time: in the past the distribution of the stopped antiprotons has been approximated using the obtained for the stopped muons while a fixed time inside the live window has been used for the annihilation time.

Fig. 4.22 shows the time distribution of $\bar{p}$ stops in the ST. Using this time as starting time of the particles produced in $\bar{p}$ annihilations it is possible to correctly evaluate the effect of the time selection cut on this kind of background.

\footnotetext{
${ }^{21}$ The Dalitz decay $\pi^{0} \rightarrow \gamma e^{+} e^{-}$has a branching ratio of $\sim 1 \%$.
} 


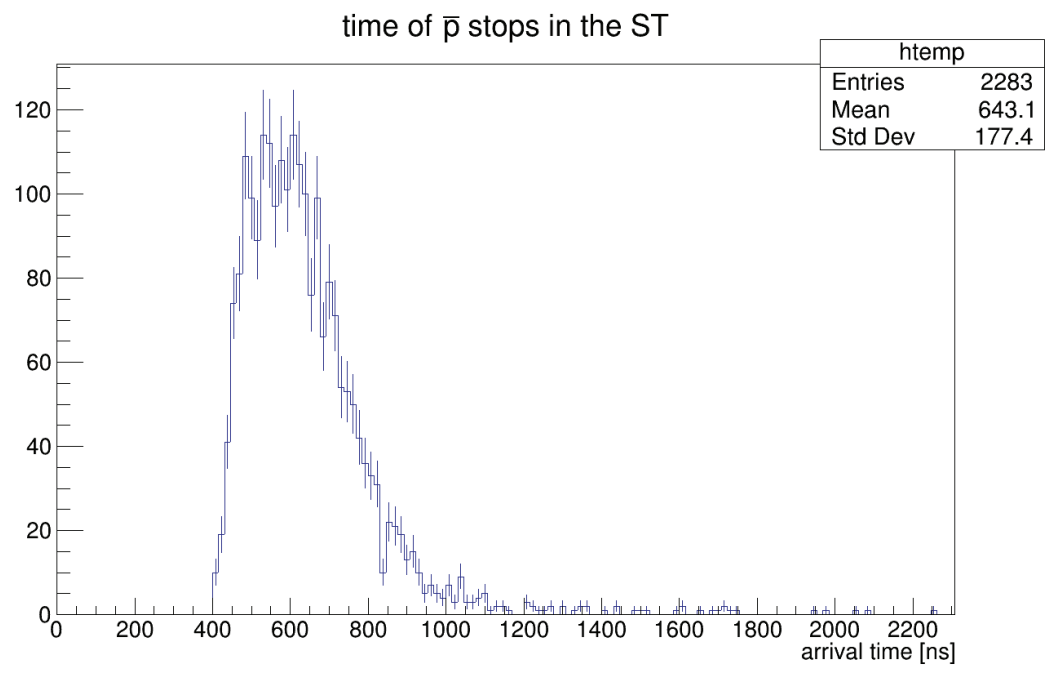

Figure 4.22: Distribution of recorded times of the antiproton stops in the Stopping Target.

\subsection{S6: Reconstruction and track selection}

The final step needed to evaluate the antiproton background is the reconstruction of the tracks in the tracker and clusters in the calorimeter. The reconstruction techniques have been described in Sec. 2.6. To be consistent with the estimates presented in Chap. 3, the same pattern recognition algorithm and fit procedure have been used (a logic $O R$ of the tracker-only and calorimeter seeded pattern recognition and the TrkCaloHit fit).

Only $50 \%$ of events originating from $\bar{p}$ and releasing hits in the tracker have a track reconstructed: the remaining half is not able to produce an helix satisfying the trigger selection. The total combined efficiency of S5 + reconstruction than is $\simeq 10 \%$.

Most of the tracks produced by $\bar{p}$ annihilations are muons coming from the $\pi^{-}$decays. This can be observed looking at the ratio E/p between the energy reconstructed in the calorimeter and the track momentum (Fig. 4.23). Continuing to be consistent with the

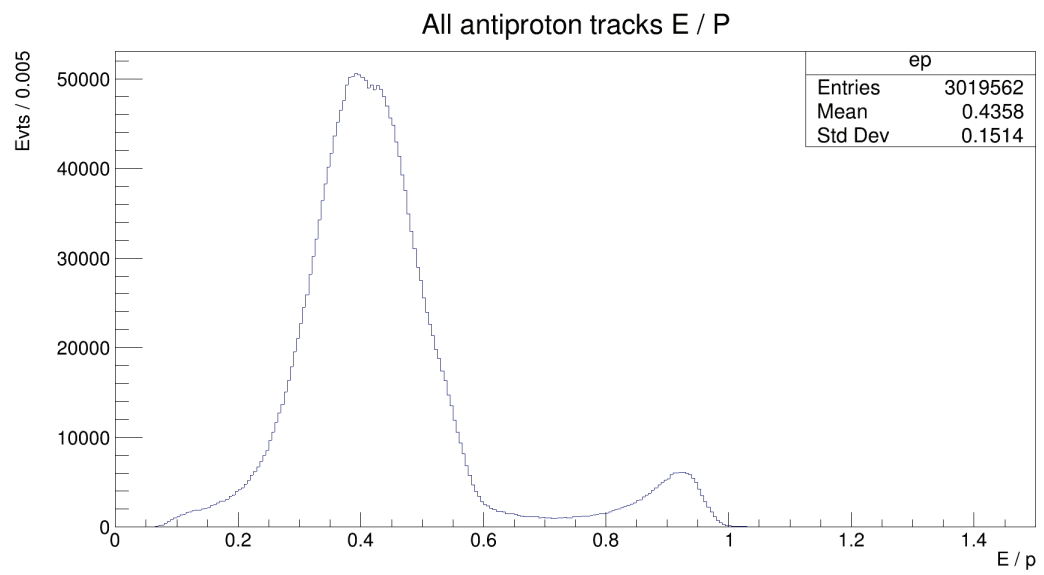

Figure 4.23: Distribution of the $\mathrm{E} / \mathrm{P}$ variable for all the reconstructed tracks originating from antiprotons.

estimates of other backgrounds, the same geometric, quality and PID cuts listed in Tab. 3.1 have been used. The combined efficiency is thus further reduced to $\simeq 1.9 \cdot 10^{-3}$. 
The final background rejection is obtained applying the momentum and time windows used for the signal selection. The distributions for the reconstructed momentum and the time at the center of the tracker $\left(T_{0}\right)$ are presented in Fig. 4.24.
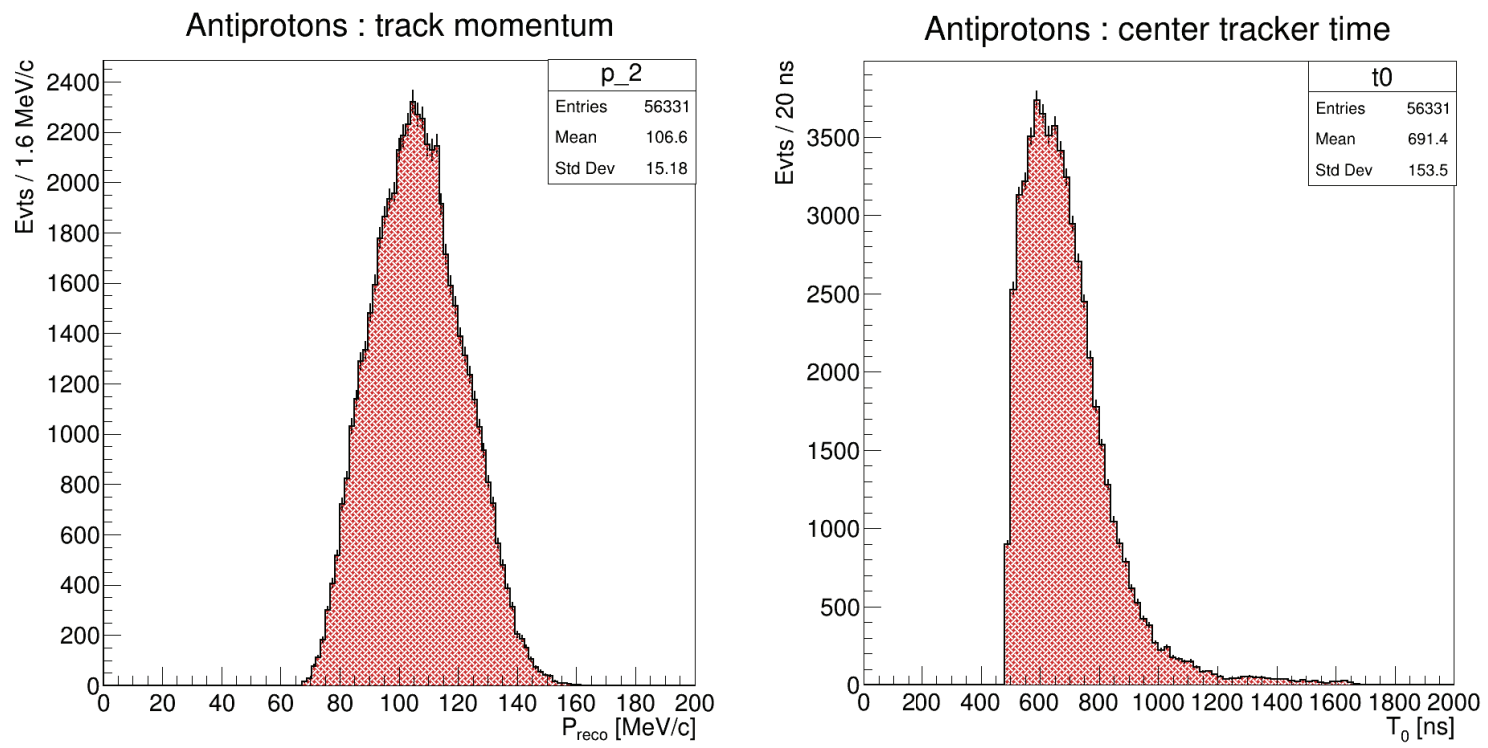

Figure 4.24: (LEFT) Reconstructed track momentum distribution for those events who satisfy the selection criteria of Tab 3.1. (RIGHT) Same events distributed in the $T_{0}$ variable, representing the reconstructed time in the geometrical center of the tracker.

For the moment we just want to estimate the number of background events corresponding to the momentum and time selection used in the CD3 background evaluation (selection in Eq. 3.13).

Out of $3 \times 10^{7} \bar{p}$ annihilations in the ST only 563 events are contained in this signal window. This corresponds to a number background electrons per stopped $\bar{p}$ :

$$
\mathcal{P}\left(e^{-} \text {reco. } \mid \bar{p} \text { stop. }\right)=(1.88 \pm 0.08(\text { stat. })) \times 10^{-5}
$$

\subsection{First estimate of antiproton background}

Combining Eq. 4.35 and 4.36 it's possible to obtain the CE background induced by antiprotons annihilating in the $\mathrm{ST}$ as function of the number of Protons on Target $N_{P O T}$ :

$$
\bar{p} \text { induced background }=N_{P O T} \times \mathcal{P}(\bar{p} \text { stop. } \mid P O T) \times \mathcal{P}\left(e^{-} \text {reco. } \mid \bar{p} \text { stop. }\right)
$$

To compare with the CD3 results we can use $N_{P O T}=3.6 \times 10^{20}$ to get:

$$
\bar{p} \text { induced background }= \begin{cases}0.044 \pm 0.003(\text { stat. }) & \text { (Striganov's model }) \\ 0.150 \pm 0.014(\text { stat. }) & \text { (Bernstein's model) }\end{cases}
$$

Statistical fluctuations between the first and the second factor are considered as uncorrelated.

For what concerns the systematic error the most important effects have been identified as: 
- The difference of the results obtained using the two cross section models that is much larger than the statistical errors. The $10 \%$ uncertainty on the Bernstein's model would have been chosen as definitive systematic uncertainty if the two models were found to be compatible ${ }^{22}$. This is not the case, and both models have tried to refine the approach to better describe experimental data regarding the backwards antiproton production that, before this work, was considered the most relevant for the background calculation. Given the lack of data for the forward production of antiprotons ( $3^{\circ}$ to $59^{\circ}$ ) with momentum above $2 \mathrm{GeV} / \mathrm{c}$, there's no indication of what model should be preferred for the extrapolation in that region. For this reason we decide to take as best guess for the antiproton induced background the average of the results obtained using the two models and assign their semi-difference as systematic uncertainty. This corresponds to a systematic error of $54 \%$.

- A relevant fraction of the antiproton background originates from antiprotons of $\sim 2 \mathrm{GeV} / \mathrm{c}$ that are back-scattered by the nuclei. The description of this process is completely determined by the model used by Geant4 to describe the hadronic inelastic interaction at these energies. There are not enough data of $\bar{p}$ scattering to validate this model. Further studies are needed to evaluate the uncertainty associated to this lack of knowledge.

- The total inelastic cross section for proton scattering on tungsten appearing in Eq. 4.34 is known with an uncertainty of $2 \%$.

- The probabilities to have a stopped antiproton presented in Eq. 4.35 have been obtained combining two different generated dataset with different generator weights and resampling factors. If the two datasets are processed separately the results differ by the $3 \%$ that is compatible with the statistical error. The systematic error induced by the resampling or by the particular function chosen to build the weighted generator appears then to be below the $1 \%$.

- As it has already been discussed in Sec. 4.7.4 the effect of the oversampling has been evaluated by replacing the content of the bins containing an antiproton resampled more than 10 times with the average occupancy of the adjacent bins: the variation on the number of stopped antiprotons is below $1 \%$.

Considering a systematic error of $54 \%$ the number of fake CE expected from $\bar{p}$ annihilations in the ST correspondent $3.6 \cdot 10^{20} \mathrm{POT}^{23}$ :

$$
\bar{p} \text { induced background }=0.097 \pm 0.008 \text { (stat.) } \pm 0.052 \text { (syst.) }
$$

using as signal windows $T_{0}>700 \mathrm{~ns}$ and $103.85<p<104.9 \mathrm{MeV} / \mathrm{c}$.

Given the entity of the systematic uncertainty, it's not worth to put at work more computing resources to reduce the statistical error.

This result is compatible with what has been quoted in the previous sensitivity estimate:

$$
\bar{p} \text { induced background }(\mathrm{CD} 3)=0.040 \pm 0.001 \text { (stat) } \pm 0.020 \text { (sys) }
$$

but comes with a deeper understanding of the systematic effects that can be critical for the measurement. An in situ measurement of the number of antiprotons stopped in the ST would significantly reduce the uncertainty connected to the theoretical models.

\footnotetext{
${ }^{22}$ This was the original intention of having an uncertainty matrix from a fit to the data.

${ }^{23}$ statistical uncertainties between the results given with different models cannot be considered completely correlated since the weights enter in the calculation.
} 


\subsection{The antiproton generated RPC component}

As it was anticipated in the introduction, a potentially dangerous background connected to the antiprotons is given by the pions produced by the antiproton interactions inside the TS: these pions arrive later with respect to the pions directly produced in the PT and can be captured in the ST creating an RPC background that is not suppressed by the time window cut.

The same procedures described for stages from S2 to $\mathrm{S} 4$ can be applied to the pions to obtain the probability to have a stopped pion in the ST generated during the transportation of an antiproton:

$$
\mathcal{P}\left(\pi^{-} \text {stop. } \mid \text { POT }\right)= \begin{cases}(4.0 \pm 0.14(\text { stat })) \times 10^{-16} & \text { (Striganov's model }) \\ (8.9 \pm 0.13(\text { stat })) \times 10^{-16} & \text { (Bernstein's model })\end{cases}
$$

The number of stopped pions is $\sim 50$ times larger than the one of stopped antiprotons: this is mainly because many $\bar{p}$ s are absorbed in the TS absorbers, in particular the titanium window located at the center of the TS, while pions can pass through to them or be produced after them.

Stage 4 records positions and times of the pions stopped in the ST. Fig. 4.25 shows the time z-coordinate of these stops. A quick comparison with Fig. 4.22 shows that pions generally reach the ST earlier with respect to antiprotons. This is justified by the fact that, given their lower mass, pions with momenta in the range accepted by the TS travel much faster than the antiprotons with the same momenta. The pion arrival time on the ST is the sum of the travel time of its parent antiproton plus its travel time: the sooner the pion is created, the earlier it reaches the ST. Two components created in different parts of the TS and reaching the ST at different times are also visible in the plot. The z-coordinate plot exhibits the bigger penetrating power of pions compared to antiprotons (Fig. 4.19).
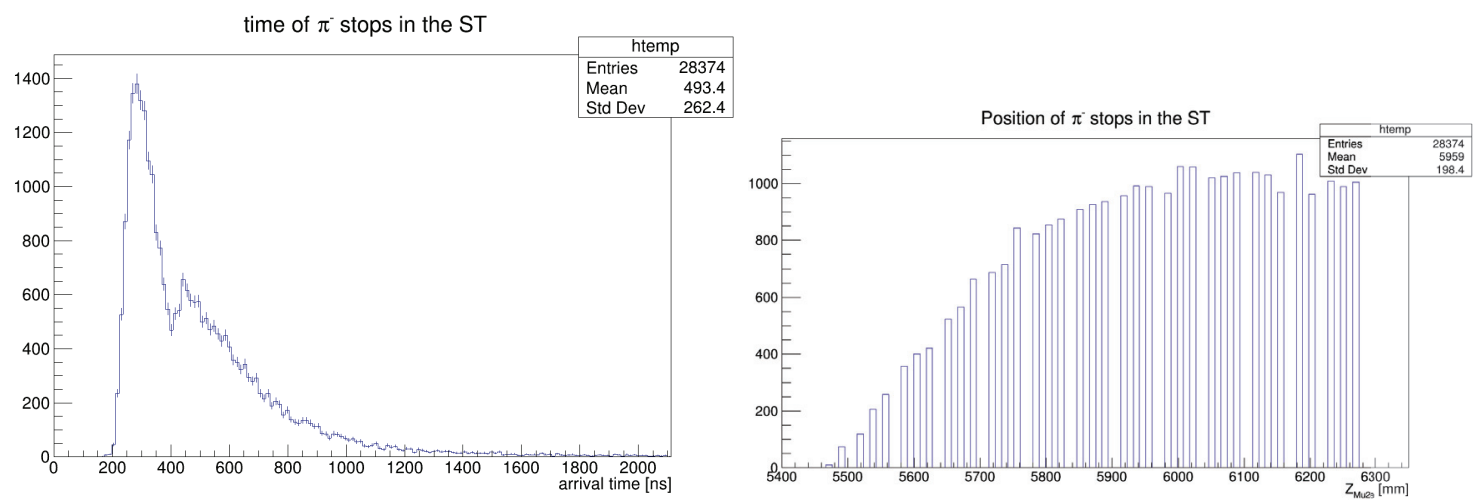

Figure 4.25: Arrival time (LEFT) and z-coordinate RIGHT distribution for all the pion stops in the ST.

Stage 5 uses the stopped pions vertices to generate the photons produced in the Radiative Pion Capture in the nucleus. The probability to have a RPC when a pion is stopped in the Aluminum is $\mathrm{BR}(\mathrm{RPC})=2.1 \times 10^{-2}$ (Bistirlich et al [63]) The photon spectrum in 3.7 has been used to generate $2 \times 10^{7}$ photons from the stopped pion vertices. The outgoing photons have been traced inside the DS to study their conversions in $e^{+} e^{-}$ pairs. 
In Stage 6 the tracks produced by the electrons have been reconstructed and filtered using the standard selection criteria (Tab. 3.1).

The momentum and center tracker time distributions of the reconstructed tracks appear as shown in Fig. 4.26 .

$\pi^{-}$from $\bar{p}:$ track momentum

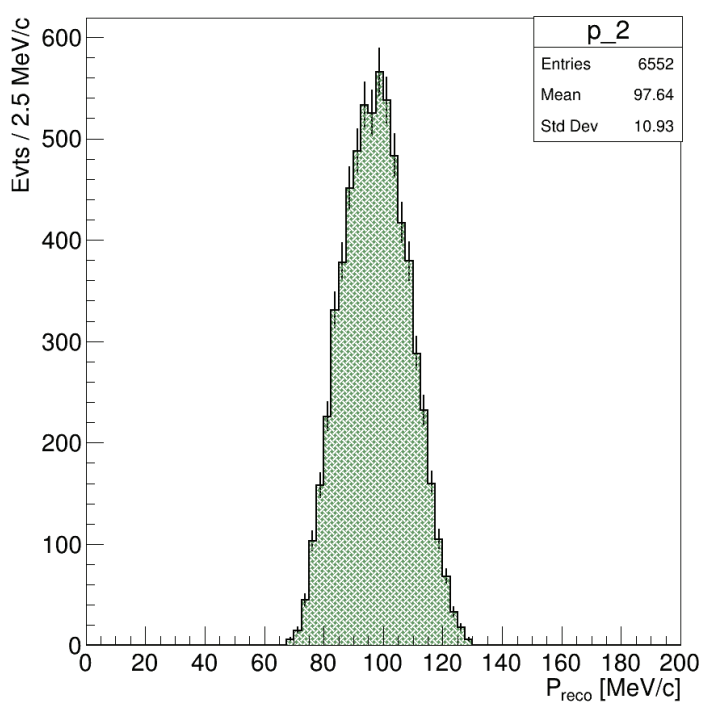

$\pi^{-}$from $\bar{p}:$ center tracker time

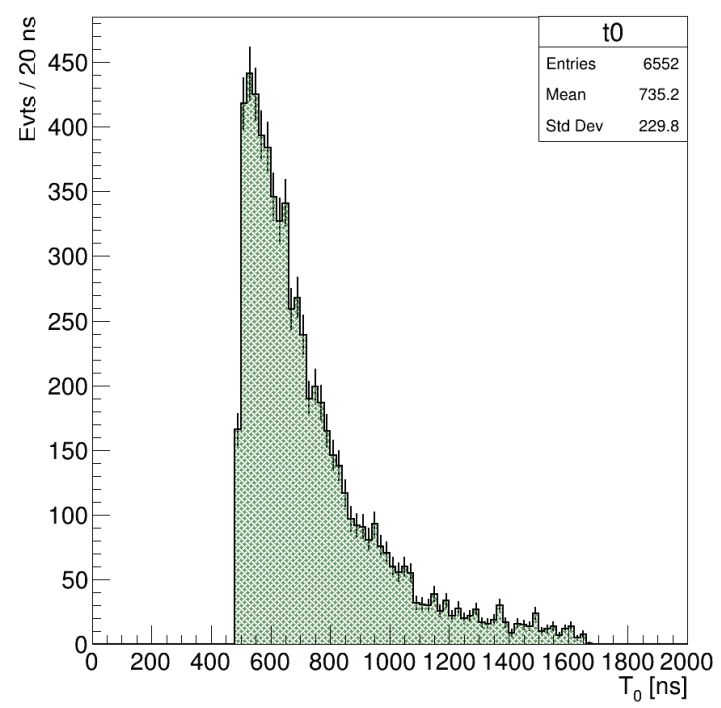

Figure 4.26: Momentum and time distribution of the reconstructed tracks coming from a special class of $\pi^{-} \mathrm{s}$ created during the transportation of a $\bar{p}$ and stopped inside the ST. The selection criteria listed in Tab. 3.1 have been applied.

Also in this case the number of CE-fake events (within the comparison with the CD3 analysis) is obtained counting the number of electron tracks passing the selection and within the CD3 momentum and time window: $103.85<p<104.9 \mathrm{MeV} / \mathrm{c}$ and $T_{0}>700$ ns. This counting corresponds to the photon conversions out of the nucleus (external conversions). A contribution of the same entity is expected from the internal conversions inside the nucleus. For this reasons a factor 2 is applied to the background produced by the external conversions.

Given the available statistics of $2 \times 10^{7}$ photons, 85 events are counted in the signal region, the number of background events per stopped $\pi^{-}$coming from S5 and S6 simulation then is (external + internal RPCs):

$$
\mathcal{P}\left(e^{-} \text {reco. } \mid \pi^{-} \text {stop. }\right)=B R(R P C) \times \frac{N_{\text {reco }}}{N_{\text {rgen }}} \times 2=(1.8 \pm 0.2) \times 10^{-7}
$$

The resulting number of background events for $N_{P O T}=3.6 \times 10^{20}$ is:

$$
\begin{gathered}
\bar{p} \text { induced RPC background }=N_{P O T} \times \mathcal{P}\left(\pi^{-} \text {stop. } \mid P O T\right) \times \mathcal{P}\left(e^{-} \text {reco. } \mid \pi^{-} \text {stop. }\right) \\
= \begin{cases}0.026 \pm 0.003 \text { (stat.) } & \text { (Striganov's model) } \\
0.058 \pm 0.006 \text { (stat.) } & \text { (Bernstein's model) }\end{cases}
\end{gathered}
$$

Finally, making the average of the results of the two models and taking their semidifference as dominant systematic error we obtain:

$$
\bar{p} \text { induced RPC background }=0.042 \pm 0.005 \text { (stat.) } \pm 0.016 \text { (syst.) }
$$


This is about one half of the background produced by the antiproton annihilations in the stopping target and cannot be neglected. 


\section{Chapter 5}

\section{Optimizing the sensitivity}

In the previous chapters, the updated results for the "CD3 run conditions" have been presented and compared with the previous results. In this final chapter we will normalize the signal and background distributions to the data statistics expected to be collected in the first two years. The momentum and time selection will then be optimized to get the best result on the sensitivity.

\subsection{Latest Mu2e run plan}

The most important aspect that requires Mu2e to update its sensitivity estimate is the change in the run plan. Since the data taking period will be interrupted by the accelerator shutdown for the neutrino beam upgrade and important detector modifications could occur in that period, we will limit the study of the expected sensitivity to the first two years of data taking.

Based on [88], Mu2e will run for 61 days with 1 batch in 2024, 120 days with 1 batch in 2025 and 36 days with 2 batches in 2025. Tab. 5.1 reports the running time, $T_{R U N}$, for the three different periods:

\begin{tabular}{|c|c|c|c|}
\hline year & $\mathrm{N}$ (batches) & $\begin{array}{c}T_{R U N} \\
\text { from }[88]\end{array}$ & $T_{E F F}(s)$ \\
\hline 2024 & 1 & 61 days & $2.45 \cdot 10^{6}$ \\
\hline 2025 & 1 & 120 days & $7.07 \cdot 10^{6}$ \\
\hline 2025 & 2 & 36 days & $1.58 \cdot 10^{6}$ \\
\hline
\end{tabular}

Table 5.1: Mu2e real and effective running time in the first two years of data taking: 2024 and 2025. The second year will host both a data taking with 1 and 2 batches per Main Injector cycle.

The effective running time $T_{E F F}$ is obtained correcting $T_{R U N}$ for multiple factor, the most impactful being the accelerator and detector uptime, the beam duty factor and transition times.

The correspondent number of delivered protons (POT) can be obtained from the effective running time using the duration of a MI cycle and the number of delivered protons per cycle. The number of spills per MI cycle will be 4 (8) for the 1 (2) batch configuration. The number of protons per spill will be $\sim 10^{12}$ in both configurations (see Sec. 2.2). The complete formula then is:

$$
N_{\text {POT exp }}=N_{\text {cycles }} \times N_{\text {POT cycle }} \quad \text { with: } N_{\text {cycles }}=\frac{T_{E F F}}{\text { cycle duration }}
$$


Tab. 5.2 finally contains the specific calculation for each batch configuration.

\begin{tabular}{|c|c|c|c|c|c|}
\hline year & $\mathrm{N}$ (batches) & $T_{E F F}(\mathrm{~s})$ & cycle duration $(\mathrm{s})$ & $N_{\text {POT cycle }}$ & $N_{\text {POT exp }}$ \\
\hline 2024 & 1 & $9.52 \cdot 10^{6}$ & 1.33 & $4 \cdot 10^{12}$ & $2.86 \cdot 10^{19}$ \\
\hline $2024-2025$ & 2 & $1.58 \cdot 10^{6}$ & 1.4 & $8 \cdot 10^{12}$ & $9.03 \cdot 10^{18}$ \\
\hline
\end{tabular}

Table 5.2: Total number of protons on target correspondent to a specific running time. Results are keeping separate for the 1 batch and 2 batches period.

The sum of the two contributions is $3.76 \cdot 10^{19}$ POT and corresponds to the total statistics that will be gathered by Mu2e before the accelerator shutdown, in the so called Run 1.

\subsection{Momentum and time distributions}

Expected momentum distributions for signal and main backgrounds, discussed and presented in Chap. 3 and Chap. 4, can now be normalized to the number of POT correspondent to the presented Run 1 plan. In the following, the small background contribution coming from RMC, decays in flight and beam-electrons will be neglected and only cosmics, DIOs, antiprotons and RPCs will be considered. They are all shown in Fig. 5.1 both keeping channels separated (non-stacked option, top plot) and stacking each contribution on top of each other (stacked option, bottom plot). The error bars include both statistical and systematic errors. The shown CE signal corresponds to $R_{\mu e}=10^{-15}$. To make the plot significant and not overwhelmed by RPCs, an indicative time cut $\mathrm{t}>$ 700 ns has been applied. This will change after the time window optimization.

DIOs give the dominant contribution below $\simeq 103 \mathrm{MeV} / \mathrm{c}$ : since their number drastically changes with the momentum, they are expected to be decisive to set the lower edge of the momentum window. Cosmics are reasonably flat in the showed region and, even if dominant in $[104,105] \mathrm{MeV} / \mathrm{c}$, they will only impact the optimization of the upper edge of the momentum selection. The other backgrounds also follow a flat distribution in the considered interval but are at least a factor 10 less relevant than cosmics.

The same can be done with the time distributions (Fig. 5.2). Again an indicative momentum cut of $[103,105] \mathrm{MeV} / \mathrm{c}$ has been applied: in this case it is evident that the only limiting factor is represented by RPC (both external and internal) background, which makes impossible any measurement below $\sim 600$ ns.

\subsection{Momentum and time selection optimization}

For those experiments looking for highly rare processes of new physics, it is common to express their discovery potential in terms of the "sensitivity". Two different sensitivity definitions are often quoted, one is relative to the actual potential of a discovery while the other expresses how tight the constraint can be set on the unknown parameter (which is most commonly the cross section of the process) in case of no significant observation. In Mu2e, both the aspects are of great importance and thus the momentum and time selection will be optimized following both approaches separately.

In the first approach the figure of merit is the sensitivity for a 5-sigma discovery, in the second the $90 \%$ CL upper limit on the conversion probability. 

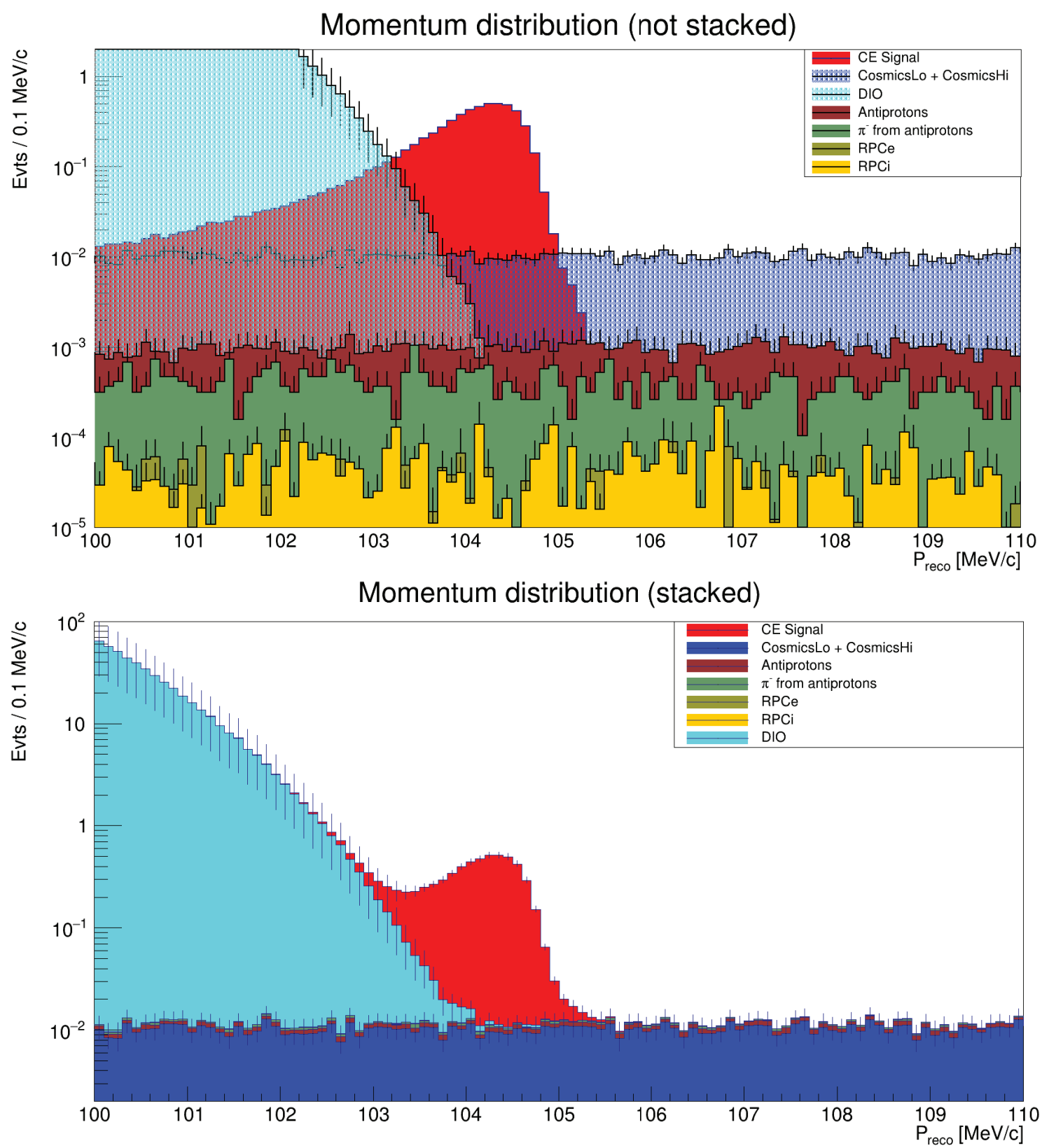

Figure 5.1: Momentum distribution for expected signal and backgrounds in non-stacked (top) and stacked (bottom) mode. Histograms are normalized to $3.76 \cdot 10^{19}$ POT. Both plots have been built with an indicative time cut at $\mathrm{t}>700 \mathrm{~ns}$ and a signal $R_{\mu e}=1 \times 10^{-15}$. No momentum selection has been applied. Error bars include both statistical and systematic errors

To avoid biases due to the choice of a specific experiment outcome, a combined approach leading to the definition of a "sensitivity region" will also be presented.

\subsection{Discovery-based optimization}

Before performing the optimization, we need to define in rigorous terms what a "discovery" is and what is its figure of merit. 


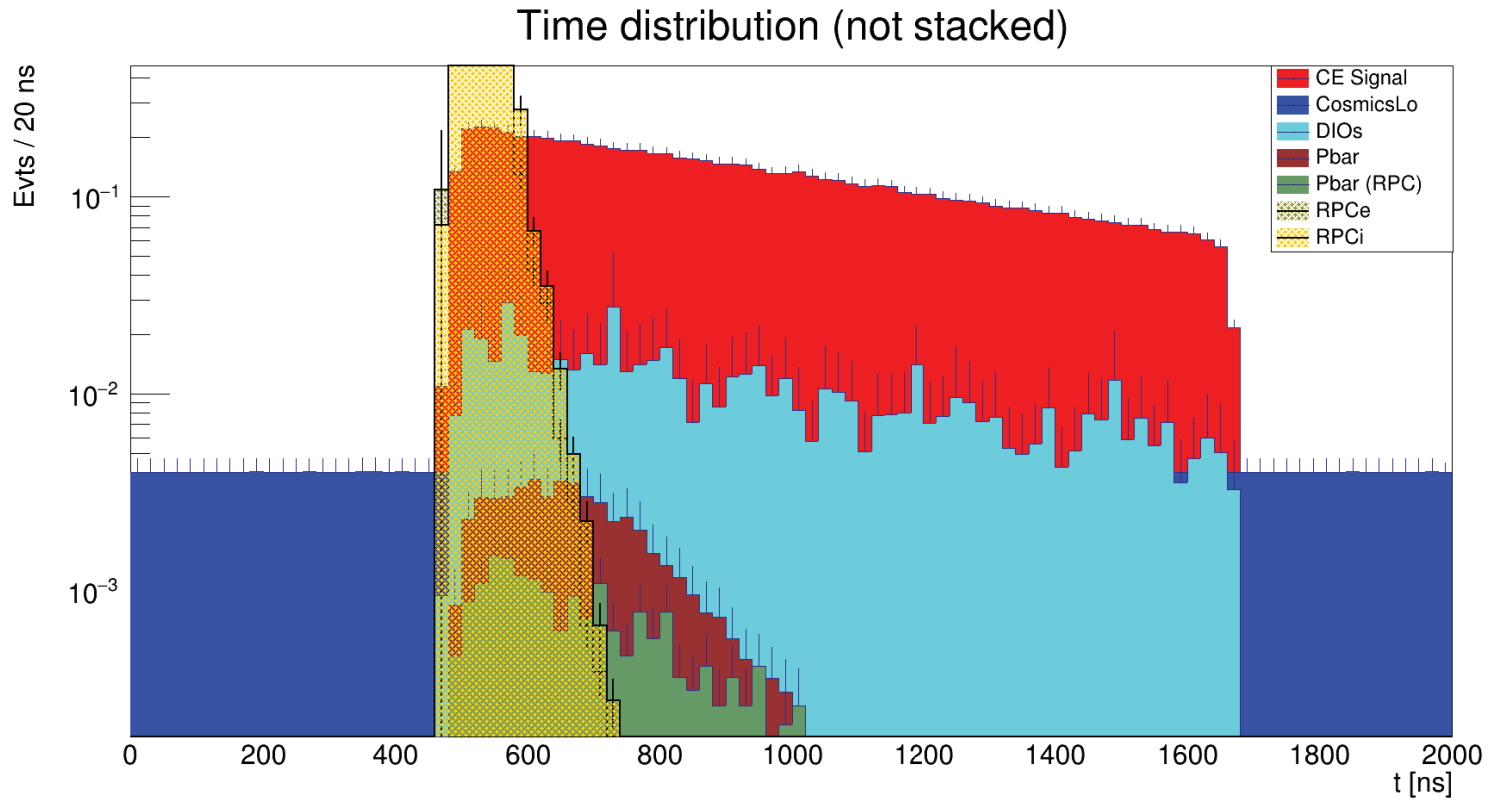

Figure 5.2: Time distribution for expected signal and backgrounds in non-stacked mode. Histograms are normalized to $3.76 \cdot 10^{19}$ POT. The plot is shown within an indicative momentum window of $[103,105] \mathrm{MeV} / \mathrm{c}$ and a signal $R_{\mu e}=1 \times 10^{-15}$ No time selection has been applied.

Let us consider an hypothesis test built to distinguish between two distinct Poisson probability density functions, both distributing the outcome $n$ of a counting experiment. The first is the background-only hypothesis $\left(H_{0}\right)$, i.e. the data can be understood with existing physics, the second one is the signal + background hypothesis $\left(H_{s}\right)$ in which new physics is needed in order to understand the observed data:

$$
p(n ; s)=\frac{(s+b)^{n} e^{-(s+b)}}{n !} \quad p(n ; s=0)=\frac{b^{n} e^{-b}}{n !}
$$

The expected number of events "s" is proportional to $R_{\mu e}$ and not known a-priori. The expected number of background events "b" can instead be estimated by the sum of the expected background yields within a certain momentum and time window.

We define now the significance of an hypothesis test $\alpha$ as the probability of rejecting $H_{0}$ when it is indeed true. For a counting experiment the critical region $\mathcal{C}$, i.e. the values of the observable $n$ for which the $H_{0}$ hypothesis is rejected with a significance $\alpha$, is expressed as:

$$
\mathcal{C} \equiv\left\{n: n>n_{5 \sigma}(\alpha)\right\}
$$

Where $n_{5 \sigma}$ is a certain threshold to be identified.

Based on the past Mu2e sensitivity update (CD3, July 2017, [52]), a "discovery" is defined as the case in which the value $R_{\mu e}=0$ (the $H_{0}$ hypothesis) is excluded at the $5 \sigma$ level. A common practice for new physics searches is to use the $5 \sigma$ probability of a Standard Gaussian, that defines $n_{5 \sigma}$ as the value for which ${ }^{1}$ :

$$
\mathcal{P}\left(n>n_{5 \sigma} \mid H_{0}\right) \leq 5.733 \cdot 10^{-7}
$$

\footnotetext{
${ }^{1}$ Because of the discrete nature of the Poisson distribution, the equality can not be exactly satisfied. In these cases it is required that the probability for an observation to land in the critical region is less or equal to $\alpha$.
} 
Since the condition is made under the $H_{0}$ hypothesis, the threshold of the test $n_{5 \sigma}$ is a function of the expected background b only, which is in turn function of the momentum and time windows. An example of how the threshold is set is shown in Fig. 5.3, for an indicative value of $b=0.1$.

\section{Poisson distribution with $\mathrm{b}=0.1$}

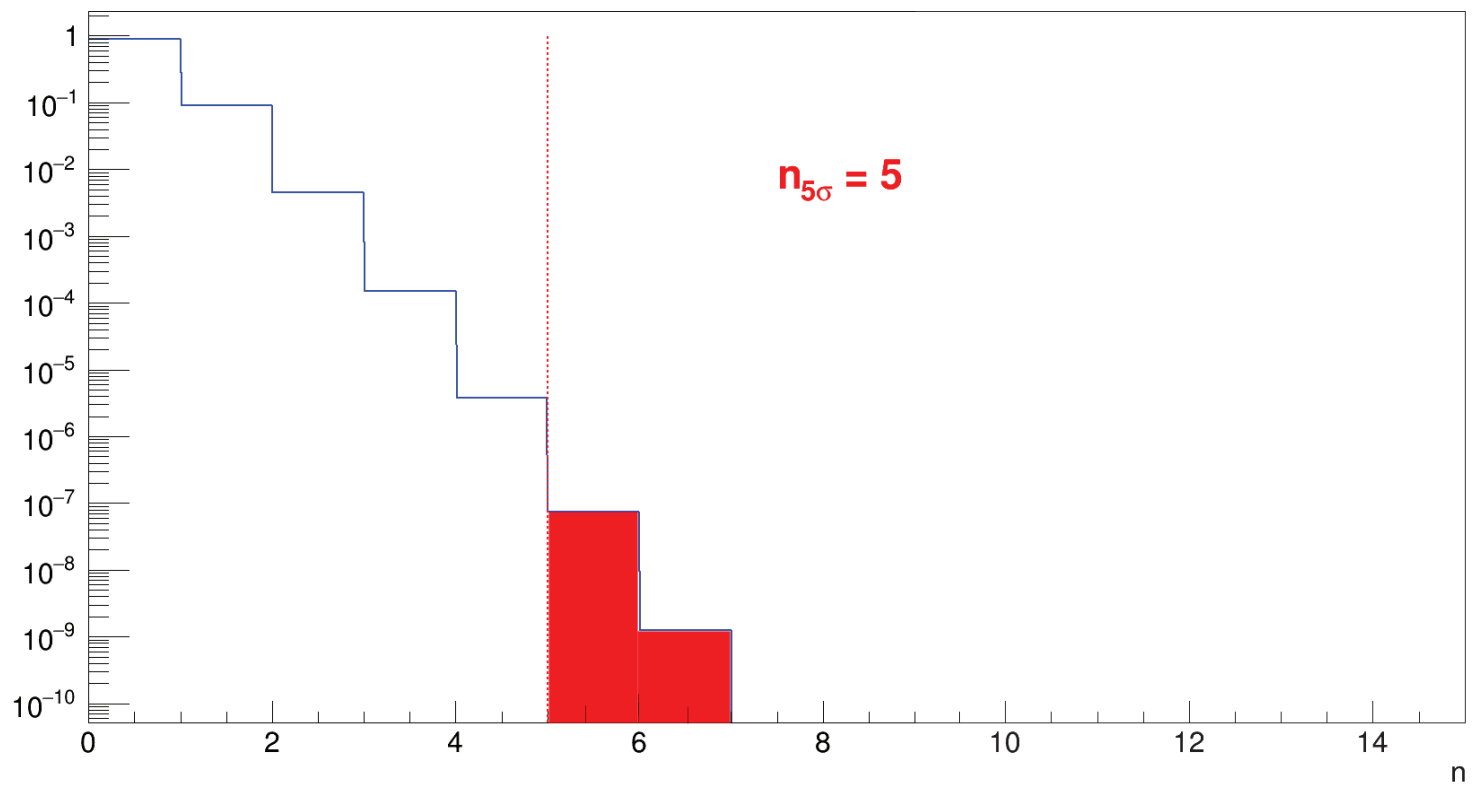

Figure 5.3: Poisson distribution in the $H_{0}$ hypothesis with an artificial value of $\mathrm{b}=0.1$. The critical region is highlighted in red and its area is $\leq 5.733 \cdot 10^{-7}$.

The other element to be considered in a test is the probability that $\mathrm{n}$ will be in the critical region (a discovery is claimed) when $H_{s}$ is true. This is a function of the unknown parameter $s$ and is expressed by the power function of the test: $\operatorname{pow}(\alpha, \mathrm{s})=1-\beta_{\alpha}(s)^{2}$.

It is possible to reproduce the shape of the power function randomly extracting $\mathrm{n}$ multiple times from the Poisson distribution under $H_{s}$, and see if each value is included or not in $\mathcal{C}$, i.e. if the corresponding experiment claims or not the discovery. Practically, for each value of $s$ a large number of "identical" experiments with the same detector, POT, analysis cuts, etc., but with the $n$ observable fluctuating is considered and the fraction of experiments in the ensemble claiming the discovery is extracted. If the chosen value of $s$ is "small", only a limited fraction of experiments will claim the discovery ${ }^{3}$. On the contrary, if the chosen $s$ is very large, most of them will extract a high $\mathrm{n}$ and reject the background-only hypothesis.

Finally, the median discovery signal $s_{5 \sigma}(\mathrm{b})$ is defined as the value of $\mathrm{s}$ for which half of the experiments will claim the discovery. Fig. 5.4 shows an example of the power plot obtained with this procedure and a scan in the signal mean parameter from 0 to 20 , always for an indicative value of $b=0.1\left(10^{5}\right.$ "experiments" have been simulated for each value of $\mathrm{s}$ ). The calculation of $s_{5 \sigma}$ is still only dependent on the background estimate (b), we ultimately want our figure of merit also te be dependent on the expected signal efficiency.

\footnotetext{
${ }^{2}$ Consequently, $\beta_{\alpha}$ is the probability not to claim a discovery when actually it would have been possible because $H_{s}$ is true

${ }^{3}$ Note that even for $s=0$ that fraction may not be zero because of the statistical fluctuations.
} 


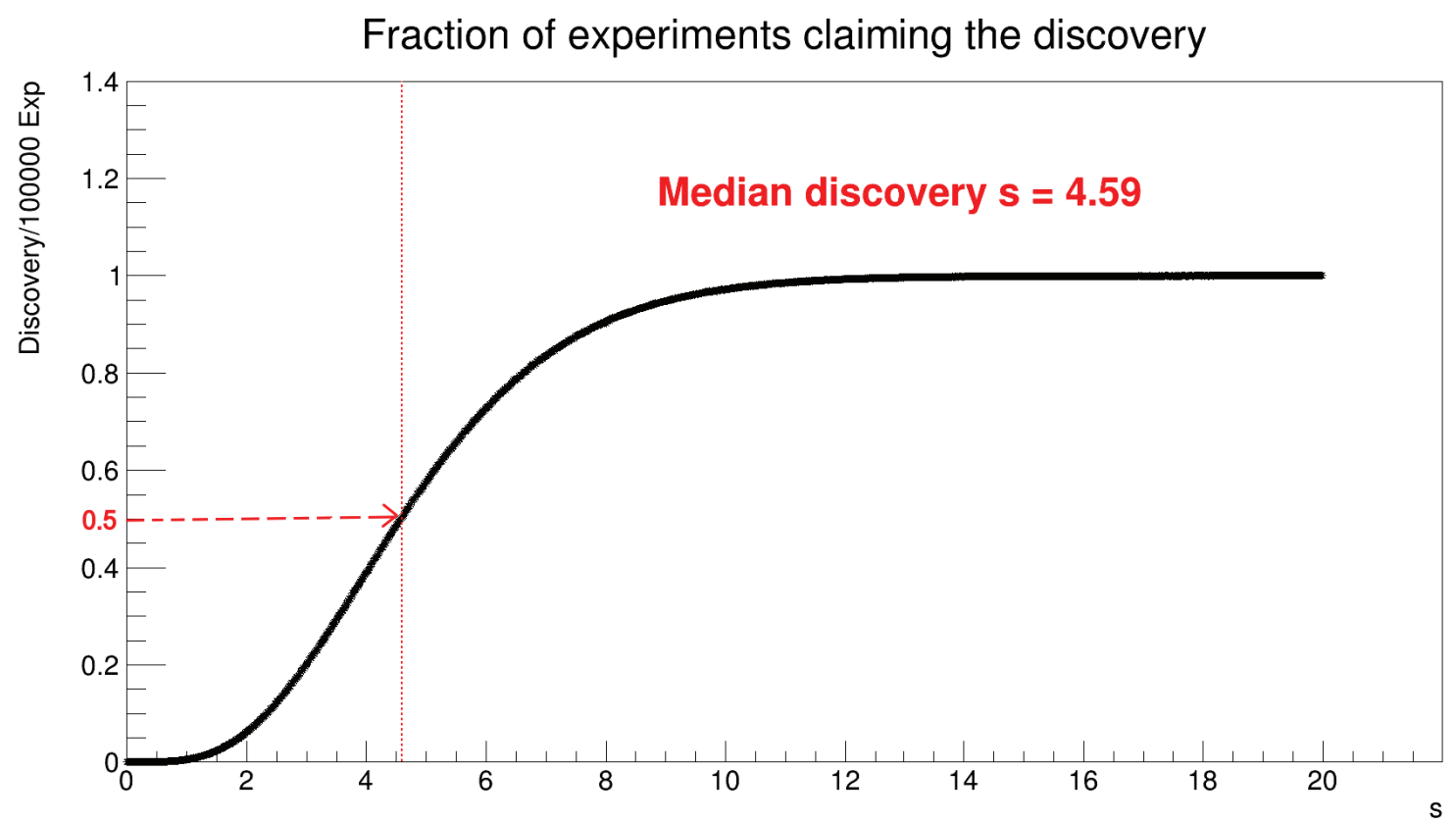

Figure 5.4: Fraction of experiments claiming the discovery if $H_{s}$ is true (power of the test) as a function of the signal mean parameter $s$.

This could be done in the following way. We first express the mean number of signal events as a function of the experiment design parameters and the branching ratio of the conversion process:

$$
s=N_{P O T} \times P_{\mu s t o p} \times B R\left(\mu^{-} \rightarrow e^{-}\right) \times \epsilon_{C E}
$$

and so:

$$
B R\left(\mu^{-} \rightarrow e^{-}\right)=\frac{s}{N_{P O T} \times P_{\mu \text { stop }} \times \epsilon_{C E}}
$$

This allows the introduction of the single event sensitivity (SES) that is the branching ratio (probability) corresponding to one expected signal event (on average) with negligible background. It can be easily obtained from the 5.5 setting $\mathrm{s}=1$ :

$$
S E S \equiv \frac{1}{N_{p} \times P_{\mu \text { stop }} \times \epsilon_{C E}}
$$

The SES can be estimated with the information coming from the MC analysis of the signal and it also depends on the time and momentum selection.

It follows that the median discovery signal $s_{5 \sigma}$ can be translated into the median discovery sensitivity $R_{\mu e} 5 \sigma$ (our figure of merit) by recalling the definition of $R_{\mu e}$ :

$$
R_{\mu e}=\frac{B R\left(\mu^{-} \rightarrow e^{-}\right)}{B R_{\text {capture }}}
$$

from which:

$$
R_{\mu e}=\frac{s \times S E S}{B R_{\text {capture }}}
$$

and ultimately:

$$
R_{\mu e ~ 5 \sigma}\left(\epsilon_{C E}, b\right) \equiv \frac{s_{5 \sigma}(b) \times S E S\left(\epsilon_{C E}\right)}{B R_{\text {capture }}}
$$


A signal efficiency dependence has then been introduced.

Different time and momentum cuts can be tested to find the smallest $R_{\mu e} 5 \sigma$. Wider windows will increase the signal efficiency and so reduce the SES, but too wide windows will bring more background than signal, make the increases of $s_{5 \sigma}$ dominant with respect to the improvement on the SES.

The momentum and time optimization is carried on simultaneously using a grid with steps of $10 \mathrm{~ns}$ and $0.05 \mathrm{MeV} / \mathrm{c}$, ranging the following intervals: $p_{\min }=[102,104] \mathrm{MeV} / \mathrm{c}$, $p_{\max }=[104,106] \mathrm{MeV} / \mathrm{c}$ and $t_{\min }=[550,800]$. There is no need to optimize the upper edge of the time window; it can in fact be extended up to the end of the available time interval between two proton pulses i.e. $1695 \mathrm{~ns}$.

The optimal windows providing the best $R_{\mu e} 5 \sigma$ are:

$$
p \in[103.5,104.8] \mathrm{MeV} / \mathrm{c} \quad t \in[630,1695] \mathrm{ns}
$$

This corresponds to a global efficiency on the CE signal of $\epsilon_{C E}=14.3 \%$ and an expected number of background events $\mathrm{b}=0.28$. The found median discovery sensitivity is:

$$
R_{\mu e ~ 5 \sigma}=1.03 \cdot 10^{-15}
$$

This is the minimum value of the $R_{\mu e}$ parameter for which the Mu2e experiment would claim a discovery in the $50 \%$ of the cases.

Using the 5.5 and the extracted efficiency in the optimized window, it is also possible to calculate the correspondent median discovery signal:

$$
s_{5 \sigma}=5.27
$$

\subsection{Limit-based optimization}

The alternative optimization fits the case where the experiment does not observe a significant difference with respect to the background-only hypothesis.

The procedure for each configuration of momentum and time selection is the following:

- The Feldman-Cousins (FC) ordering [89] is used to build the $90 \%$ confidence belts (Fig. 5.5). This must be preferred with respect to the decreasing $n$ ordering to set the upper limit and avoiding empty confidence intervals in regions of low counting.

- An ensemble of $10^{4}$ experiments is simulated, in which the number of observed events $(n)$ is extracted from a Poisson distribution of mean $b$ and the upper edge of the $90 \%$ belt for the parameter $s$ is saved.

- The mean of these saved values is taken as the mean $90 \%$ upper limit on $s$.

- Finally, as in the previous optimization, the upper limit on $s$ is converted with the usage of the SES into the upper limit on $R_{\mu e}$, which is used as the figure of merit.

Results of the optimization are:

$$
p \in[103.25,104.9] \mathrm{MeV} / \mathrm{c} \quad t \in[600,1695] \mathrm{ns}
$$

corresponding to $\epsilon_{C E}=16.4 \%$ and $\mathrm{b}=0.62$. The $90 \% \mathrm{CL}$ upper limit is:

$$
R_{\mu e}<4.9 \cdot 10^{-16} \quad(s<2.87)
$$

The optimized intervals are slightly different from the ones obtained from the discoverybased method: wider windows are preferred when a limit has to be set in case of no signal evidence. 


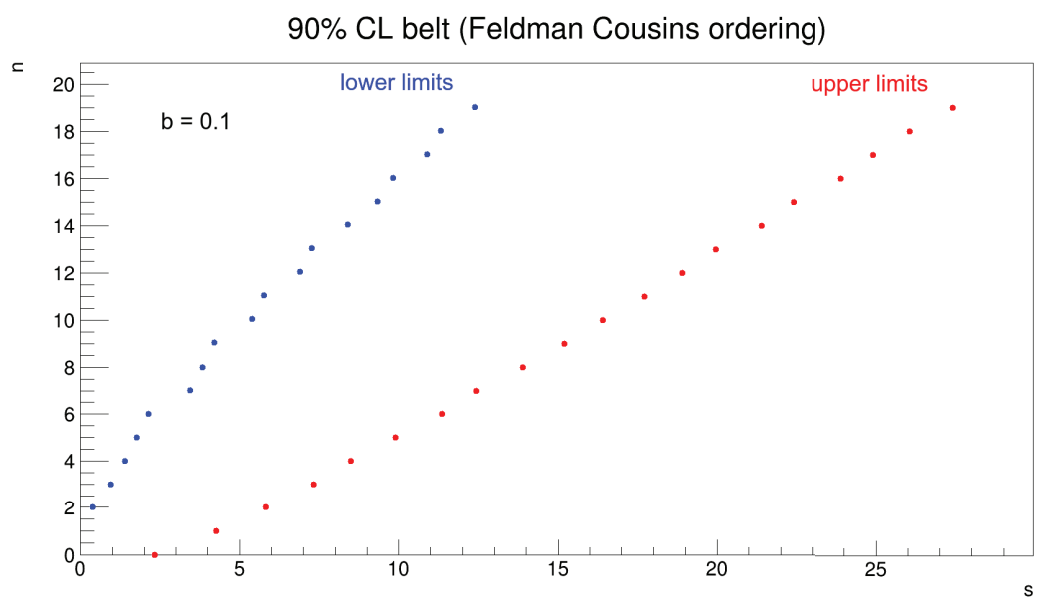

Figure 5.5: 90\% Confidence Level band for a Poisson of bean $b=0.1$. The $\mathrm{x}$-axis contains the upper (Red) and lower (Blue) limits on the signal mean parameter while the y-axis contains the possible values for the observable $n$.

\subsection{A combined approach: the sensitivity region}

In principle, the discovery-driven approach and the set of the upper limit are completely independent. Only after the experiment is made, one can know which must be the strategy to follow to quote the most significant result. Moreover, setting a limit at a confidence level lower than the level of significance required for a discovery, can lead to undesired situations where the deviation is not enough to claim the discovery but the null signal hypothesis $H_{0}$ is not included in the allowed CL region. For example, this would happen for $\mathrm{n}=3$ in Fig. 5.5, where the FC algorithm sometimes returns a lower limit $\neq 0$.

It is interesting to look at the problem from a different point of view and present results that are unbiased by the particular ending of the experiment.

Given a fixed confidence level CL (90\%) and test significance $\alpha$ (gaussian $5 \sigma$ ) it is possible to define the "sensitivity region" $\mathcal{S}$ as the region of the test parameter $s$ for which the power of the chosen test is greater of equal to the CL chosen for the limits in case there is no discovery $([90])$ :

$$
\mathcal{S}=\left\{s: 1-\beta_{\alpha}(s) \geq C L\right\}
$$

This has two interesting properties:

- If the true value of $s$ is inside $\mathcal{S}$, the probability of claiming a discovery is at least CL.

- In case of non-significant result, the region excluded at the desired Confidence Level will contain $\mathcal{S}$ (regardless of the true value of $s$ ).

This can be used as a unified figure of merit for the optimization, requiring $\mathcal{S}$ to be the largest possible.

The method becomes very simple in case of a counting experiment. From Fig. 5.4 it can be seen that the region 5.15 correspond to the values of $s$ bigger than a certain value $s_{\min }$. The sensitivity region than takes the form:

$$
s \geq s_{\min }(b)
$$


Minimize $s_{\min }$ (i.e. maximize $\mathcal{S}$ ) coincides with what has been done for the discovery-based optimization but looking for $1-\beta \geq C L$ instead of $1-\beta=0.5$ used in the median discovery signal definition, i.e. at least a CL fraction of experiments claiming the discovery instead of $50 \%$. With this simple redefinition of the figure of merit we can unify the hypothesis test and the FC upper limit and take advantage of the two presented properties.

The optimization has then been redone and, as might be expected, confirms the previous discovery-optimized results (Fig. 5.6):

$$
p \in[103.5,104.8] \mathrm{MeV} / \mathrm{c} \quad t \in[630,1695] \mathrm{ns}
$$

but it differs in the way the result is presented. It leads to a sensitivity region of:

$$
\mathcal{S}_{\max }(5 \sigma, 90 \% \mathrm{CL})=\left\{R_{\mu e}>1.72 \cdot 10^{-15}\right\} \quad\left(s_{5 \sigma}^{90 \%}>8.85\right)
$$

This represents the region of the parameter space to which the experiment is "sensitive", i.e. is able to either claim a discovery or exclude it.
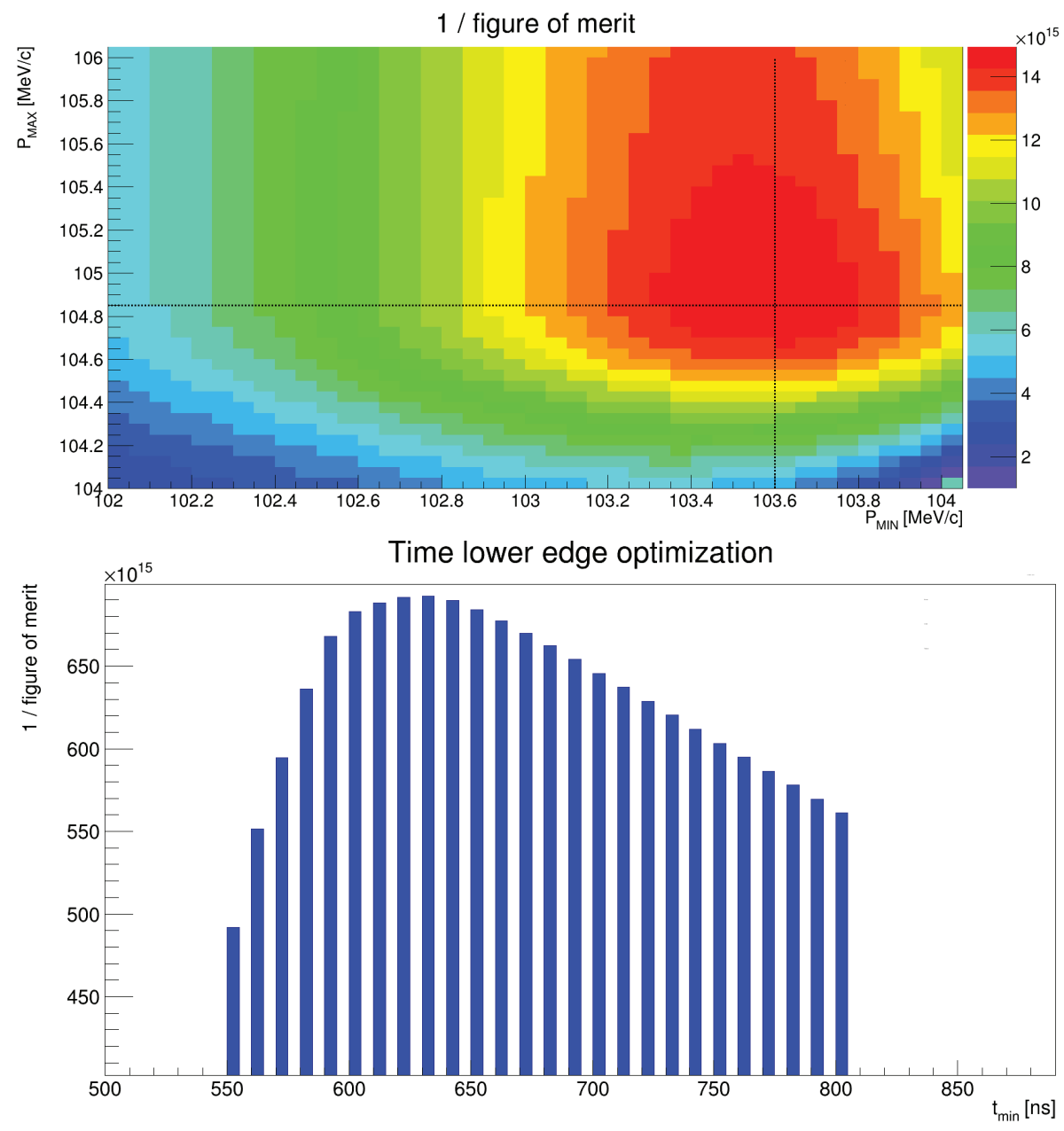

Figure 5.6: Optimization in case of the combined approach (same as discovery optimized). The plots shows the values of $1 . / R_{\mu e}$ for different choices of the lower and upper edge of the momentum cut $\left(p_{\min }\right.$ and $\left.p_{\max }\right)$ and lower end of the momentum window $\left(t_{\min }\right)$. Since the 3D space is not readable, the $2 \mathrm{D}$ projection on the momentum variables (top) and the $1 \mathrm{D}$ projection on the time variable (bottom) are shown. 


\subsection{Final background and signal expectations}

In the optimized window 5.17 the expected $\mathrm{CE}$ efficiency and number of background events are:

$$
\epsilon_{C E}=14.3 \% ; \quad \mathrm{b}=0.28
$$

Single background contributions composing the value of $b$ are shown in Tab. 5.3 (expectation are there presented with respect to the new run plan), where the right column reports the main systematic source for each background.

Varying the mean background value around $1 \sigma$ (stat. + syst.) it is possible to get an idea on the uncertainty of the presented result: the lower limit of the sensitivity region is observed to vary within $\sim 10 \%$ (from $1.6 \cdot 10^{-15}$ to $1.8 \cdot 10^{-15}$ ).

To conclude, Fig. 5.7 shows the expected final background versus reconstructed track momentum. A signal distribution for an indicative $R_{\mu e}=1 \cdot 10^{-15}$ is also shown and the momentum signal region is highlighted. Uncertainties shown for the total background include both statistical and systematic contributions.

\begin{tabular}{c|c|c}
\hline Process & SU2020 estimates, RUN 1 & Comments \\
\hline \hline Cosmics & $0.14 \pm 0.02$ (stat.) \pm 0.03 (syst.) & CRV light yield \\
\hline DIO & $0.087 \pm 0.005$ (stat.) \pm 0.068 (syst.) & Tracker scale and resolution \\
\hline Antiprotons & $0.020 \pm 0.002$ (stat.) \pm 0.011 (syst.) & unknown $\bar{p}$ production cross section \\
\hline RPC $($ from $\bar{p})$ & $0.0073 \pm 0.0006$ (stat.) \pm 0.0028 (syst.) & unknown $\bar{p}$ production cross section \\
\hline RPC & $0.027 \pm 0.003$ (stat.) \pm 0.005 (syst.) & proton pulse timing \\
\hline \hline Total & $0.28 \pm 0.02$ (stat.) \pm 0.08 (syst.) & \\
\hline
\end{tabular}

Table 5.3: Expected background contributions in the signal region $p \in[103.5,104.8] \mathrm{MeV} / \mathrm{c} \quad t \in$ $[630,1695]$. Results are bench-marked for the Mu2e Run 1, i.e. two years (2024-2025) at reduced intensity with a limited period at full intensity. The corresponding POT statistics is: $3.76 \cdot 10^{19}$ POT. 


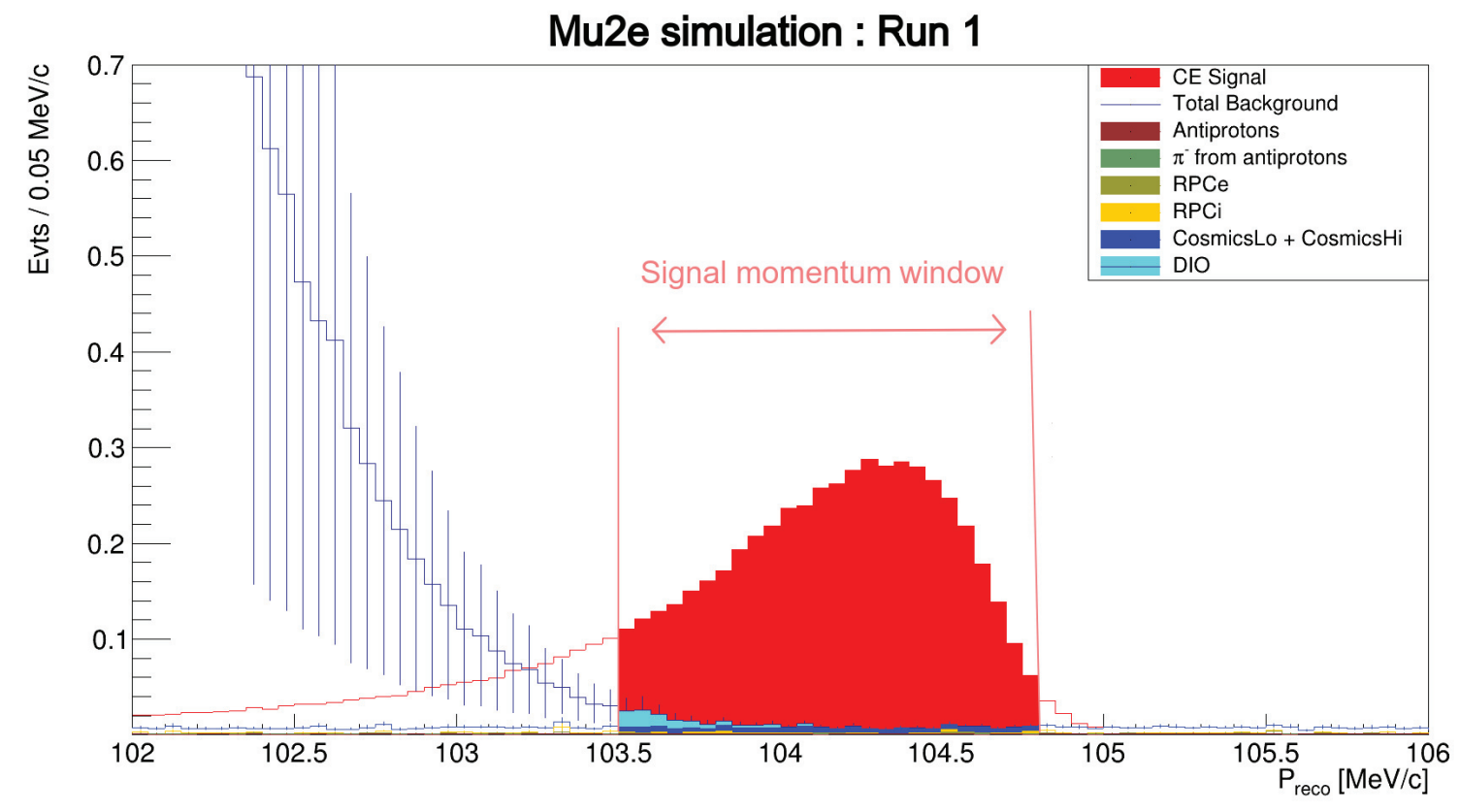

Figure 5.7: Total signal $\left(R_{\mu e}=1 \cdot 10^{-15}\right)$ and background yield and as a function of the reconstructed momentum. The highlighted region correspond to the optimized momentum signal window: $p \in[103.5,104.8] \mathrm{MeV} / \mathrm{c}$. Histograms are normalized to the total Mu2e Run 1 statistics of $3.76 \cdot 10^{19}$ POT. 


\section{Conclusions}

The work in this thesis has presented an updated estimate of the best sensitivity that the Mu2e experiment will be able to achieve in its search for the CLFV process:

$$
\mu^{-} N \longrightarrow e^{-} N
$$

The previous "blessed" estimate dates back to the time of the CD3, in 2017. A new estimate was required since the Mu2e run plan has evolved from a 3 consecutive years run at full intensity, to a staged configuration: 2 years at $\sim$ half intensity with reduced concrete shielding, a shutdown of 2 years and then 2 or more years at full intensity. Other significant updates have been introduced: the change of the experiment Production Target, a more realistic knowledge of the performances of the Cosmic Ray Veto and the improvements of the reconstruction algorithms.

To obtain the result, updated Monte Carlo datasets have been produced for both conversion electrons and main background sources (cosmic rays, decays in orbit, antiprotons and radiative pion captures) and signal selection criteria have been identified.

In the context of the Mu2e Sensitivity Update 2020 (SU2020) campaign, this work has been focused on the evaluation of the antiproton background. The result for the first two years of data taking is:

$$
\bar{p} \text { induced background }=0.020 \pm 0.002 \text { (stat.) } \pm 0.011 \text { (syst.) }
$$

representing the $7 \%$ of the total expected background. This study has revealed this background to be dominated by a particular class of events: antiprotons of $\sim 2 \mathrm{GeV} / \mathrm{c}$ initially produced forward and then back-scattered by the Production Target material. The reliability of this process is completely linked to the model used by Geant 4 to describe the hadronic inelastic interaction of low energy $\bar{p} s$, where no experimental data are available. The experiment has made this problem his own and it is now working to improve the understanding of this process. The dominant contribution to the quoted systematic error comes from the poor knowledge of the antiprotons production cross-section from 'low energies' protons on heavy-nuclei, for which the data pool is very limited. An alternative cross-section model has been built and has allowed an estimate of a systematic uncertainty when compared to the existing model. A proposal has been made to operate an in situ measurement of the number of antiprotons stopped in the Stopping Target and considerably reduce the systematic uncertainty on the production and back-scattering theoretical models.

With all datasets available, this thesis concludes with the optimization of the momentum and time selection to reach the largest possible sensitivity region, i.e. a definition that combines together the 5-sigma discovery approach and the set of the upper limit in case of no signal. The study has revealed that, with the update understanding of the experiment, wider momentum and time windows with respect to CD3 can be used:

$$
p \in[103.5,104.8] \mathrm{MeV} / \mathrm{c} \quad t \in[630,1695] \mathrm{ns}
$$


that translate into the following sensitivity region for the $R_{\mu e}$ parameter:

$$
\mathcal{S}_{\max }(5 \sigma, 90 \% \mathrm{CL})=\left\{R_{\mu e}>1.72 \cdot 10^{-15}\right\}
$$

The above result is referred to the first 2 years of data taking and has the following property: if the real value of $R_{\mu e}$ is inside $\mathcal{S}$, Mu2e will be able to claim a 5 -sigma discovery $90 \%$ of the times, if it is lower, it will exclude at least $\mathcal{S}$ at $90 \%$ CL. In this last case an ad-hoc analyses with even wider momentum and time windows gives to the best $90 \% \mathrm{CL}$ upper limit:

$$
R_{\mu e}<4.9 \cdot 10^{-16}
$$

This result will improve the previous SINDRUM II limit by a factor 1400 . 


\section{Bibliography}

[1] ATLAS collaboration, "Observation of a new particle in the search for the Standard Model Higgs boson with the ATLAS detector at the LHC," Physics Letters B, vol. 716, p. 1-29, Sep 2012.

[2] CMS collaboration, "Observation of a new boson at a mass of $125 \mathrm{GeV}$ with the CMS experiment at the LHC," Physics Letters B, vol. 716, p. 30-61, Sep 2012.

[3] S. Bifani, S. Descotes-Genon, A. R. Vidal, and M.-H. Schune, "Review of lepton universality tests in B decays," Journal of Physics G: Nuclear and Particle Physics, vol. 46, Dec 2018.

[4] LHCb collaboration, "Test of lepton universality with $\Lambda_{b}^{0} \rightarrow p K^{-} \ell^{+} \ell^{-}$decays," Journal of High Energy Physics, May 2020.

[5] LHCb collaboration, "Search for Lepton-Universality Violation in $B^{+} \rightarrow K^{+} l^{+} l^{-}$ Decays," Physical Review Letters, vol. 122, May 2019.

[6] LHCb collaboration, "Measurement of the ratio of branching fractions $\mathcal{B}\left(\bar{B}^{0} \rightarrow\right.$ $\left.D^{*+} \tau^{-} \bar{\nu}_{\tau}\right) / \mathcal{B}\left(\bar{B}^{0} \rightarrow D^{*+} \mu^{-} \bar{\nu}_{\mu}\right)$," Phys. Rev. Lett., vol. 115, no. 11, 2015. [Erratum: Phys.Rev.Lett. 115, 159901 (2015)].

[7] E. J. Konopinski and H. M. Mahmoud, "The Universal Fermi Interaction," Phys. Rev., vol. 92, pp. 1045-1049, Nov 1953.

[8] R. M. Fonseca, "Violation of lepton number in 3 units," Journal of Physics: Conference Series, vol. 1586, p. 012003, aug 2020.

[9] Y. Fukuda et al. , "Super-Kamiokande Collaboration," Phys. Rev. Lett. 82, 1999.

[10] Q. R. Ahmad et al., "SNO Collaboration," Phys. Rev. Lett. 89, 2002.

[11] K. Eguchi et al., "KamLAND Collaboration," Phys. Rev. Lett. 90, 2003.

[12] L. Calibbi and G. Signorelli, "Charged lepton flavour violation: an experimental and theoretical introduction," La Rivista del Nuovo Cimento, vol. 41, pp. 71-174, 2018.

[13] T. Cheng and L. Li, "Gauge Theory of Elementary Particle Physics," Physics Today, vol. 38, Jan 1985.

[14] W. J. Marciano, T. Mori, and J. M. Roney, "Charged lepton flavour violation experiments," Annual Review of Nuclear and Particle Science, vol. 58, pp. 315-341, 2008. 
[15] A. de Gouvêa and N. Saoulidou, "Fermilab's Intensity Frontier," Annual Review of Nuclear and Particle Science, vol. 60, no. 1, pp. 513-538, 2010.

[16] M. Raidal, A. Van Der Schaaf, I. Bigi, M. Mangano, Y. Semertzidis, S. Abel, S. Albino, S. Antusch, E. Arganda, B. Bajc, et al., "Flavour physics of leptons and dipole moments," The European Physical Journal C, vol. 57, no. 1-2, pp. 13-182, 2008.

[17] Y. Kuno and Y. Okada, "Muon decay and physics beyond the Standard Model," Reviews of Modern Physics, vol. 73, no. 1, p. 151, 2001.

[18] A. de Gouvêa and P. Vogel, "Lepton flavour and number conservation, and physics beyond the Standard Model," Progress in Particle and Nuclear Physics, vol. 71, p. 75-92, Jul 2013.

[19] E. P. Hincks and B. Pontecorvo, "Search for Gamma-Radiation in the 2.2-Microsecond Meson Decay Process," Phys. Rev., vol. 73, pp. 257-258, Feb 1948.

[20] M. Conversi, E. Pancini, and O. Piccioni, "On the Disintegration of Negative Mesons," Phys. Rev., vol. 71, pp. 209-210, Feb 1947.

[21] A. Baldini et al., "Search for the lepton flavour violating decay $\mu^{+} \rightarrow \mathrm{e}^{+} \gamma$ with the full dataset of the MEG experiment," Eur. Phys. J. C, vol. 76, no. 8, p. 434, 2016.

[22] U. Bellgardt and G. Otter and R. Eichler and L. Felawka and C. Niebuhr and H.K. Walter and W. Bertl and N. Lordong and J. Martino and S. Egli and R. Engfer and Ch. Grab and M. Grossmann-Handschin and E.A. Hermes and N. Kraus and F. Muheim and H. Pruys and A. Van Der Schaaf and D. Vermeulen, "Search for the decay $\mu^{+} \longrightarrow e^{+} e^{-} e^{+}, " N u c l e a r$ Physics B, vol. 299, no. 1, pp. $1-6,1988$.

[23] C. Dohmen et al., "Test of lepton flavour conservation in $\mathrm{mu} \rightarrow$ e conversion on titanium," Phys. Lett. B, vol. 317, pp. 631-636, 1993.

[24] W. H. Bertl et al., "A Search for muon to electron conversion in muonic gold," Eur. Phys. J. C, vol. 47, pp. 337-346, 2006.

[25] A. Baldini, E. Baracchini, C. Bemporad, F. Berg, M. Biasotti, G. Boca, P. Cattaneo, G. Cavoto, F. Cei, M. Chiappini, et al., "The design of the MEG II experiment," The European Physical Journal C, vol. 78, no. 5, pp. 1-60, 2018.

[26] V. Gilinsky and J. Mathews, "Decay of Bound Muons," Phys. Rev., vol. 120, pp. 14501457, Nov 1960.

[27] T. Suzuki, D. F. Measday, and J. Roalsvig, "Total Nuclear Capture Rates for Negative Muons," Phys. Rev. C, vol. 35, p. 2212, 1987.

[28] A. Czarnecki, X. Garcia i Tormo, and W. J. Marciano, "Muon decay in orbit spectra for $\mu-e$ conversion experiments," Hyperfine Interact., vol. 210, no. 1-3, pp. 19-23, 2012.

[29] A. Badertscher et al., "Search for $\mu^{-} \rightarrow e^{-}$conversion with SINDRUM-II," 1991. 
[30] B. Aubert, Y. Karyotakis, J. P. Lees, V. Poireau, E. Prencipe, X. Prudent, V. Tisserand, J. Garra Tico, E. Grauges, M. Martinelli, and et al., "Searches for Lepton Flavour Violation in the Decays $\tau \rightarrow e \gamma$ and $\tau \rightarrow \mu \gamma$," Physical Review Letters, vol. 104, Jan 2010.

[31] Y. Miyazaki, I. Adachi, H. Aihara, K. Arinstein, V. Aulchenko, T. Aushev, A. Bakich, V. Balagura, E. Barberio, A. Bay, and et al., "Search for lepton flavour violating $\tau$ decays into three leptons," Physics Letters B, vol. 660, p. 154-160, Feb 2008.

[32] T. Abe et al., "Belle II Technical Design Report," 2010.

[33] E. Kou, P. Urquijo, W. Altmannshofer, F. Beaujean, G. Bell, M. Beneke, I. I. Bigi, F. Bishara, M. Blanke, C. Bobeth, and et al., "The Belle II Physics Book," Progress of Theoretical and Experimental Physics, vol. 2019, Dec 2019.

[34] D. Ayres et al., "The NOvA Technical Design Report," Oct 2007.

[35] S. Werkema, "Mu2e Accelerator Timeline with a Single Proton Batch," Mu2e Internal Doc. Database, doc-db 25923-v3, May 2019.

[36] S. Werkema, "Proton Pulse Intensity Variation," Mu2e Internal Doc. Database, doc-db 32620-v2, April 2020.

[37] L. Bartoszek et al., "Mu2e Technical Design Report," arXiv:1501.05241, October 2014.

[38] E. Prebys, "Mu2e Extinction Channel," Mu2e Internal Doc. Database, doc-db 33323v1, May 2020.

[39] S. Werkema, "Mu2e Proton Beam Longitudinal Structure," Mu2e Internal Doc. Database, doc-db 27r1, February 2019.

[40] K. Lynch, "Production Target: Issues and Plans," Mu2e Internal Doc. Database, doc-db 24232, Feb 2019.

[41] D. R. Pushka, "Production Target (Hayman 2)-Requirements and Design," Mu2e Internal Doc. Database, doc-db 30814-v2, Jan 2020.

[42] J. JD, "Classical Electrodynamics," Cambdridge University Press, 1975.

[43] P. Murat, "Optimization of TS1/TS3 $\bar{p}$ absorbers," Mu2e Internal Doc. Database, doc-db 33410-v4, June 2020.

[44] J. Miller and S. Krave, "Mu2e Stopping Target, Requirements and Specifications," Mu2e Internal Doc. Database, doc-db 1437 v7, May 2019.

[45] N. Atanov et al., "The Mu2e Calorimeter Final Technical Design Report," 2018.

[46] Eleonora Diociaiuti, University of Roma Tor Vergata, "Study of the Mu2e sensitivity to the $\mu$ to $e^{+}$conversion process," PhD thesis, 2020.

[47] Gianantonio Pezzullo, University of Pisa, "The Mu2e crystal calorimeter and improvements in the $\mu^{-} N \rightarrow e^{-} N$ search sensitivity," PhD thesis, 2015. 
[48] Nam Tran (Boston University), for the STM team , "Status of the Stopping Target Monitor," Mu2e Internal Doc. Database, doc-db 33563, June 2020.

[49] J. M. for the STM group, "Introduction to the STM System," Mu2e Internal Doc. Database, doc-db 34650-v2, Jul 2020.

[50] M. V. Srivastav, "Efficiency Analysis of the Layers and the Module," Mu2e Internal Doc. Database, doc-db 27601-v5, Jul 2019.

[51] S. B. E. Craig Dukes, Ralf Ehrlich and Y. Oksuzian, "Mu2e CRV Staged Efficiency," Mu2e Internal Doc. Database, doc-db 28266-v4, Jul 2018-2020.

[52] A. Gaponenko, "Summary of backgrounds for CD3," Mu2e Internal Doc. Database, doc-db 7464 , July 2017.

[53] R. Szafron, "Radiative corrections - conversion and DIO spectrum," Mu2e Internal Doc. Database, doc-db 7615-v1, June 2016.

[54] G. Mengyun, C. Jun, et al., "Muon Simulation at the Daya Bay Site.," https://escholarship.org/uc/item/6jm8g76d, Dec. 2010.

[55] J. Beringer, et al., "Particle Data Group," Phys. Rev. D86, 010001, 2012.

[56] C. Hagmann, D. Lange, and D. Wright, "Cosmic-ray shower generator (CRY) for Monte Carlo transport codes," in 2007 IEEE Nuclear Science Symposium Conference Record, vol. 2, pp. 1143-1146, 2007.

[57] R. Szafron and A. Czarnecki, "High-energy electrons from the muon decay in orbit: Radiative corrections," Physics Letters B, vol. 753, 052015.

[58] R. Szafron and A. Czarnecki, "Bound muon decay spectrum in the leading logarithmic accuracy," Physical Review D, vol. 94, Sep 2016.

[59] P. Murat, "Including radiative corrections into DIO momentum spectrum parametrization," Mu2e Internal Doc. Database, doc-db 5610, 2015.

[60] J. A. Bistirlich, K. M. Crowe, A. S. L. Parsons, P. Skarek, and P. Truöl, "Photon Spectra from Radiative Absorption of Pions in Nuclei," Phys. Rev. C, vol. 5, pp. 18671883, Jun 1972.

[61] N. M. Kroll and W. Wada, "Internal Pair Production Associated with the Emission of High-Energy Gamma Rays," Phys. Rev., vol. 98, pp. 1355-1359, Jun 1955.

[62] D. Joseph, "Electron Pair Creation in pi- p Capture Reactions from Rest," Nuovo Cim., vol. 16, p. 997, 1960.

[63] S. Skupsky, "Radiative pion capture in 12c," Physics Letters B, vol. 36, no. 4, pp. 271 $-274,1971$.

[64] J. Brock, D. N. Brown, J. Leibson, B. Mode, "Radiative Pion Capture Backgrounds in the Mu2e Experiment," Mu2e Internal Doc. Database, doc-db 8923-v8, May 2017.

[65] I. Logashenko, "Background from beam electrons (CD3 version)," Mu2e Internal Doc. Database, doc-db 6464, Dec. 2015. 
[66] D. Armstrong et al., "Radiative muon capture on Al, Si, Ca, Mo, Sn, and Pb," Phys. Rev. C, vol. 46, pp. 1094-1107, 1992.

[67] P. M. E. Diociaiuti (LNF-INFN), M. Mackenzie (Northwestern), "Understanding the $k_{\text {MAX }}$ fits of the RMC spectra," Mu2e Internal Doc. Database, doc-db 22262, Apr. 2018.

[68] A. Edmonds, "Minimizing the DIO Background by Selecting High-Quality Measurements with Machine Learning Algorithms," Mu2e Internal Doc. Database, doc-db 33493-v2, Jun. 2020.

[69] D. Brown and A. Edmonds, "CD3 DIO background estimate and uncertainties," Mu2e Internal Doc. Database, doc-db 8219, 2017.

[70] A. Crescini, University of Pisa, "Master degree thesis," 2019.

[71] "CORSIKA, latest version 7.7400 (May 28, 2020)," hhttps://www.ikp.kit.edu/corsika/.

[72] "FLUKA," http://www.fluka.org/fluka.php.

[73] G. Cowan, "Statistical tests with weighted Monte Carlo events," Physics Department, Royal Holloway, University of London, Egham, TW20 0EX, U.K, May 2012.

[74] J.F. Amann et al, "Measurement of Production Cross Sections for Negative Pions, Kaons, and Protons at 10,18 and 24 GeV," Los Alamos National Laboratory, Oct 1982.

[75] Sibirtsev, A A, Safronov, G A, Smirnov, G N, and Trebukhovskij, Yu V, "Antiproton production in proton-nucleus interactions at $10.1 \mathrm{GeV} /$ c.," Yadernaya Fizika; (USSR), vol. $53: 1$.

[76] Yu. T. Kiselev et al, "Probing of compact baryonic configurations in nuclei in $p A \rightarrow \bar{p} X$ reactions and antiproton formation length in nuclear matter," Physical Review $C$, 054904 (2012), May 2012.

[77] Barabash et al, "Measurement of the cross-section $d^{2} \sigma / d p d \Omega$ in the reaction $p A \rightarrow \bar{p} X$ at $10 \mathrm{GeV} / \mathrm{c}$ proton momenta in Be, Al, Cu and Au nuclei," Durham HEPData Project, Jan 2017.

[78] S.V. Boyarinov et al, "Yields of $p, \bar{p}, \pi^{ \pm}$, and $K^{ \pm}$Emitted at an Angle of 97 degree in the Laboratory System from Nuclei Irradiated by $10.14 \mathrm{GeV}$ Protons," Physics of Atomic Nuclei Vol.57 No.8, 1994, Jul 1993.

[79] S. Striganov, "Pbar Background Studies with MARS," Mu2e Internal Doc. Database, doc-db 1776-v3, Jul 2011.

[80] L. Tan and L. Ng, "Parameterization of hadron inclusive cross-section in p p collisions extended to very low-energies.," J. Phys. G, vol. 9, pp. 1289-1308, 1983.

[81] F. Taylor, J. Carey, J. Sauer, and R. Walker, "Analysis of radial scaling in singleparticle inclusive reactions," Phys. Rev. D, vol. 14, 091976. 
[82] J.R. Letaw, R. Silberberg, C.H. Tsao, "Proton-nucleus total inelastic cross sections: An empirical formula for energies bigger than $10 \mathrm{MeV} ., "$ Astrophysical Journal, 271-276., 1983.

[83] R. Bernstein, "A New Antiproton Production Model," Mu2e Internal Doc. Database, doc-db 27801-v10, Jul 2019.

[84] S. S. Shimanskiy, "Cumulative processes out off the nucleus fragmentation region," Nov 2014.

[85] G.A. Leksin, "Methods for investigating nuclear matter under the conditions characteristic of its transition to quark-gluon plasma," M Phys. Atom. Nuclei 65 pp 1985-1994, vol. 65, February 2002.

[86] CERN, "The Geant 4 toolkit," https://geant4.web.cern.ch/.

[87] Green C. and Kowalkowski J. and Paterno M. and Fischler M. and Garren L. and Lu Q., "The Art Framework," J. Phys. Conf. Ser., vol. 396, p. 022020, 2012.

[88] G. Rakness, "Assumptions for Run-1 run plan," Mu2e Internal Doc. Database, doc-db 33731-v1, Jun. 2020.

[89] G. J. Feldman and R. D. Cousins, "Unified approach to the classical statistical analysis of small signals," Physical Review D, vol. 57, p. 3873-3889, Apr 1998.

[90] G. Punzi, "Sensitivity of searches for new signals and its optimization," Proceedings of PHYSTAT2003: Statistical Problems in Particle Physics, Astrophysics, and Cosmology, 092003. 